University of Louisville

ThinkIR: The University of Louisville's Institutional Repository

Electronic Theses and Dissertations

8-2004

\title{
Nucleation and growth studies of crystalline carbon phases at nanoscale.
}

Radhika C. Mani 1978-

University of Louisville

Follow this and additional works at: https://ir.library.louisville.edu/etd

\section{Recommended Citation}

Mani, Radhika C. 1978-, "Nucleation and growth studies of crystalline carbon phases at nanoscale." (2004). Electronic Theses and Dissertations. Paper 899.

https://doi.org/10.18297/etd/899

This Doctoral Dissertation is brought to you for free and open access by ThinkIR: The University of Louisville's Institutional Repository. It has been accepted for inclusion in Electronic Theses and Dissertations by an authorized administrator of ThinkIR: The University of Louisville's Institutional Repository. This title appears here courtesy of the author, who has retained all other copyrights. For more information, please contact thinkir@louisville.edu. 
NUCLEATION AND GROWTH STUDIES OF CRYSTALLINE CARBON PHASES AT NANOSCALE

By

Radhika C. Mani

B.E., Maharaja Sayajirao University, Baroda, India, 2000

\author{
A Dissertation \\ Submitted to the Faculty of the \\ Graduate School of the University of Louisville \\ in Partial Fulfillment of the Requirements \\ for the Degree of
}

Doctor of Philosophy

Department of Chemical Engineering

University of Louisville

Louisville, Kentucky 40292

August 2004 
NUCLEATION AND GROWTH STUDIES OF CRYSTALLINE CARBON PHASES AT NANOSCALE

\section{By}

Radhika C. Mani

B.E., Maharaja Sayajirao University, Baroda, India, 2000

A Dissertation Approved on

July 6, 2004

By the Following Dissertation Committee:

$\overline{\text { Dr. Mahendra K. Sunkara (Dissertation Director) }}$

Dr. Richard P. Baldwin

Dr. Thomas L. Starr

Dr. Stephen Rankin

Dr. Shi-Yu Wu 


\section{DEDICATION}

This dissertation is dedicated to my parents

$$
\text { Rama Chandrasekhar Mani }
$$

and

Chandrasekhar Mani

who have been my source of motivation and strength throughout this journey. 


\section{ACKNOWLEDGMENTS}

I would like to acknowledge my dissertation advisor, Dr. Mahendra Sunkara, for his guidance and encouragement throughout my PhD. His optimism, enthusiasm and pace in research have been truly captivating. I gratefully acknowledge Speed Scientific School and Chemical Engineering Department at University of Louisville for financially supporting my graduate education with the Grosscurth fellowship and teaching assistantship. I thank all the committee members, Dr. Starr, Dr. Baldwin, Dr. Rankin, and Dr. Wu for their time and input in reviewing my dissertation.

I am very grateful to one of my collaborators (late) Prof. John Cowley from Arizona State University (ASU) for his valuable assistance with electron diffraction and stimulating discussions on analyzing them. I thank all my collaborators Dr. R. P. Baldwin (University of Louisville), Dr. K. Rajan (Rensselaer Polytechnic Institute), Dr. A. M. Rao (Clemson University), and Dr. M. O'Keeffe (ASU) for their significant contributions. I am thankful for being given the access to the supercomputing facility at the CVIP lab. My work at the CVD and MR Lab would not be half as enjoyable without my fellow members Shashank, Hari, Gopi, Hongwei, Sreeram and Rodica.

A special thanks to Shashank for always being there, and encouraging me to put my best foot forward. I am deeply indebted to my family Amma, Appa, Girija, Jiju, and Mallika for their incessant support and blessings throughout my graduate studies. Amma and Appa have never failed to appreciate my small successes and cheer me up through my failures. I would have never made it this far without them. 


\title{
ABSTRACT
}

\section{NUCLEATION AND GROWTH STUDIES OF CRYSTALLINE CARBON PHASES}

\author{
AT NANOSCALE \\ Radhika C. Mani
}

August 6, 2004

Understanding the nucleation and early stage growth of crystals from the vapor phase is important for realizing large-area single-crystal quality films, controlled synthesis of nanocrystals, and the possible discovery of new phases of materials. Carbon provides the most interesting system because all its known crystalline phases (diamond, graphite and carbon nanotubes) are technologically important materials. Hence, this dissertation is focused on studying the nucleation and growth of carbon phases synthesized from the vapor phase.

Nucleation experiments were performed in a microwave plasma chemical vapor deposition (CVD) reactor, and the resulting carbon nanocrystals were analyzed primarily using electron nanodiffraction and Raman spectroscopy. These studies led to the discovery of two new crystalline phases of $\mathrm{sp}^{3}$ carbon other than diamond: face-centered and body-centered cubic carbon. Nanodiffraction results revealed possible hydrogen substitution into diamond-cubic lattices, indicating that these new phases probably act as intermediates in diamond nucleation. Nucleation experiments also led to the discovery of two new morphologies for $\mathrm{sp}^{2}$ carbon: nanocrystals of graphite and tapered, hollow 1-D structures termed here as "carbon nanopipettes". 
A Kinetic Monte Carlo (KMC) algorithm was developed to simulate the growth of individual diamond crystals from the vapor phase, starting with small clusters of carbon atoms (or seeds). Specifically, KMC simulations were used to distinguish the kinetic rules that give rise to a star-shaped decahedral morphology compared to decahedral crystals. KMC simulations revealed that slow adsorption on the $\{111\}$ steppropagation sites compared to kink sites leads to star-decahedral crystals, and higher adsorption leads to decahedral crystals.

Since the surfaces of the nanocrystals of graphite and nanopipettes were expected to be composed primarily of edge-plane sites, the electrochemical behavior of both these materials were investigated with compounds requiring chemisorption, specifically biologically important species. Both these materials exhibited a stable and reversible voltammetric behavior for dopamine (a neurotransmitter) similar to that of graphite edge planes. Furthermore, a simple bottom-up concept utilizing the tapered morphology of the nanopipettes was developed to assemble a nanoarray sensor for fast cyclic voltammetry.

In summary, the main outcomes of this dissertation include: the discovery of new crystalline carbon phases, understanding kinetic faceting of multiply twinned diamond crystals and tapered morphologies of carbon nanotubes, and development of new electrode materials based on $\mathrm{sp}^{2}$ carbon nanocrystals for sensing biologically important analytes. 


\section{TABLE OF CONTENTS}

\section{PAGE}

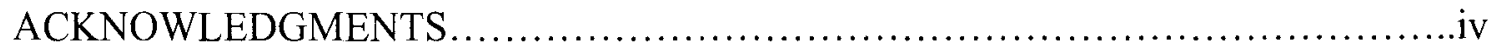

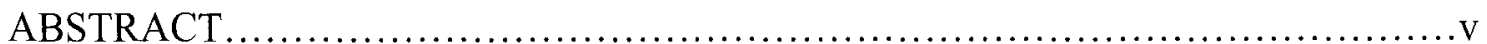

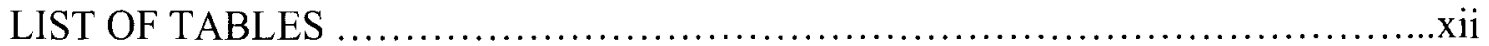

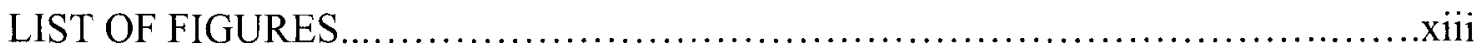

CHAPTER

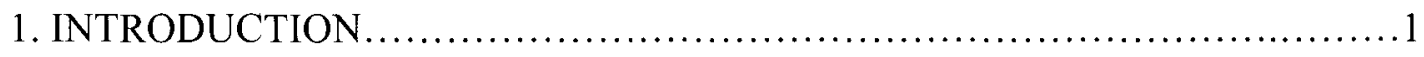

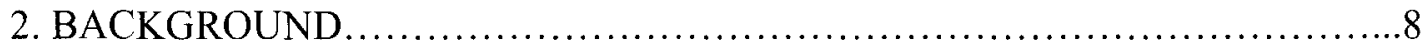

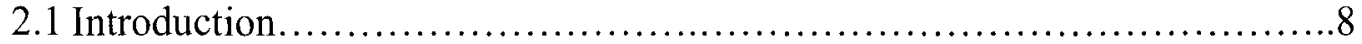

2.2 Low pressure synthesis of diamond from the vapor phase..................8

2.3 Other polymorphs and unusual phases of crystalline carbon................13

2.4 Chemistry of diamond growth from the vapor phase .......................16

2.5 Morphology of diamond crystals ....................................20

2.6 Role of defects in the morphology of diamond crystals.....................21

2.7 Simulations for kinetic modeling of large-scale crystal growth..............26

2.8 Morphological manifestations of $\mathrm{sp}^{2}$ carbon crystals........................28

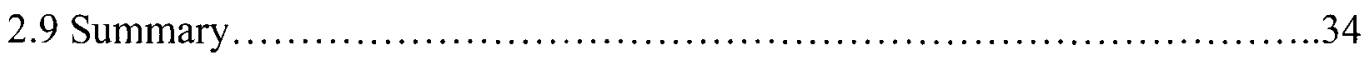

3. EXPERIMENTAL AND ANALYTICAL PROCEDURES......................36

3.1 Introduction ........................................................... 
3.2 Nucleation experiments

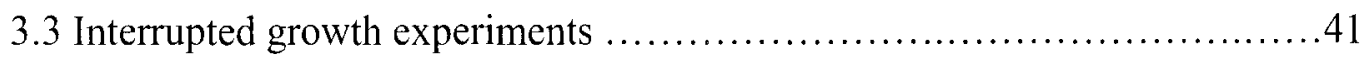

3.4 Nanomaterials characterization....................................... 42

3.4.1 Electron Microscopy....................................44

3.4.1.1 Scanning electron microscope (SEM) and

Electron dispersive X-ray spectroscopy (EDS) ..........45

3.4.1.2 Transmission electron microscope..................46

3.4.1.2 .1 Nanodiffraction using an STEM..............47

3.4.1.2.2 Simulations of electron diffraction using

Mac Tempas $\mathbb{B}$ and Crystal Kit $\mathbb{B}$

3.4 .2 X-ray diffraction............................................. 51

3.4.3 Atomic force microscopy ................................... 52

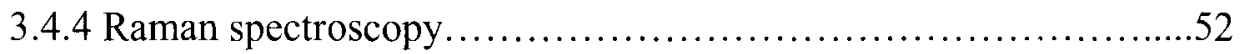

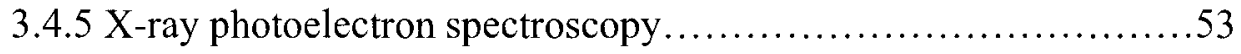

3.5 Electroanalytical characterization..................................... 55

3.5.1 Cyclic voltammetry.....................................56

3.5.2 Electrochemical impedance spectroscopy......................59

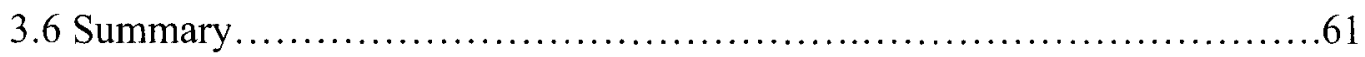

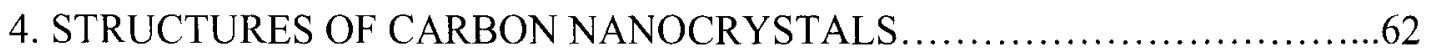

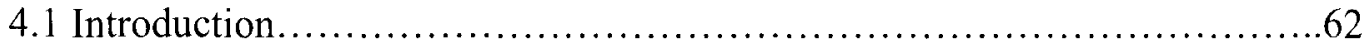

4.2 Results from nucleation experiments................................64

4.3 Structures of carbon crystals analyzed by diffraction.....................66

4.4 Understanding the n-diamond (fec) structure by electron 
diffraction simulations

4.5 Intensity ratio calculations from diffraction patterns ......................78

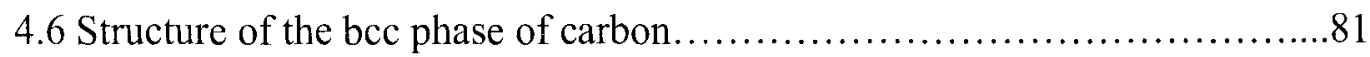

4.7 Possibility of hydrogen incorporation in carbon lattice.......................83

4.8 Energetic stability of the new carbon phases with hydrogen incorporation... 85

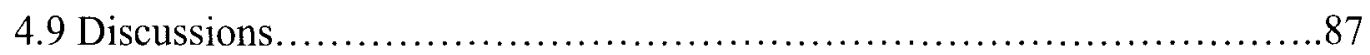

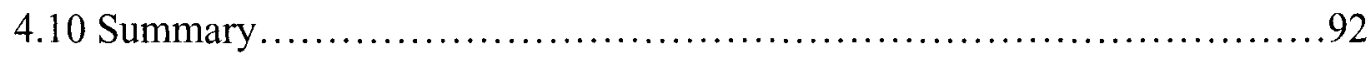

5. KINETIC FACETING OF MULTIPLY TWINNED DIAMOND CRYSTALS

DURING VAPOR PHASE SYNTHESIS ...................................99

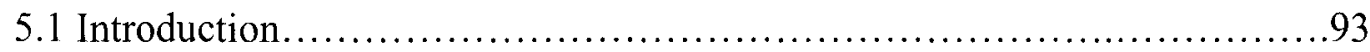

5.2 Experimental studies on the morphology evolution of twinned

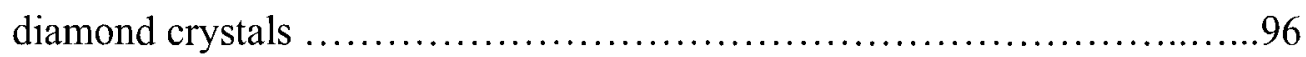

5.2.1 Results from interrupted growth experiments.....................96

5.2.2 AFM studies on star-decahedral diamond crystals.................102

5.3 Simulations for kinetic faceting of multiply twinned diamond crystals.....104

5.3.1 Simulation methodology .................................... 104

5.3.1.1 Assumptions on surface chemistry....................104

5.3.1.2 Type of surface sites................................ 106

5.3.1.3 Reaction rate constants.......................... 112

5.3.1.4 Algorithm........................................113

5.3.1.5 Time increment..................................117

5.3.1.6 Seeds used in the simulation........................118

5.3.2 Results on morphologies of computer grown crystals.............119 


\subsubsection{Simulations using a single adatom}

adsorption/desorption.

5.3.2.2 Simulations with finite etching of doubly-

bonded atoms

5.3.2.3 Simulations with increased site-specific chemistry......126

5.3.3 Discussions on $\mathrm{KMC}$ results 136

5.4 Summary. 146

6. KINETIC FACETING OF CARBON NANOTUBES

- CARBON NANOPIPETTES

6.1 Introduction. 147

6.2 Synthesis of carbon nanopipettes .148

6.3 Structural characterization.

6.4 Growth mechanism of nanopipettes: early stages of growth

6.5 Nanostructures grown on gold substrates

6.6 Summary 166

\section{ELECTROANALYTICAL SURFACE CHEMISTRY ON}

NANOCRYSTALLINE GRAPHITE MORPHOLOGIES .167

7.1 Introduction. .167

7.2 Nanocrystalline graphite films: synthesis and characterization. 170

7.3 Electroanalytical chemistry at NCG 177

7.3.1 Working potential window in aqueous solutions 178

7.3.2 Background current and double layer capacitance 179

7.3.3 Ferricyanide redox reactions 180 
7.3.4 Detection of catechols and quinones

7.3.5 Dopamine redox reactions

7.3.6 Effect of anodic pretreatment for some electrodes.

7.3.7 X-ray photoelectron spectroscopy (XPS) investigations

7.3.8 Electrode capacitance measurements.

7.3.9 Stability of electrochemical response.

7.3.10 Selectivity in mixtures

7.4 Carbon nanopipettes as electrochemical sensors .200

7.5 Nanoarray sensor 210

7.6 Summary. .214

8. CONCLUSIONS .215

9. FUTURE SCOPE

9.1 New phases of carbon. .219

9.2 KMC simulations for other materials .220

9.3 Smart drug-delivery systems using carbon nanopipettes. .221

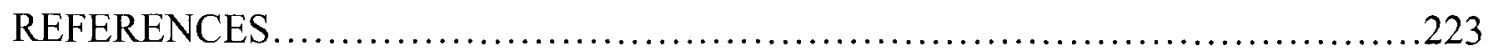

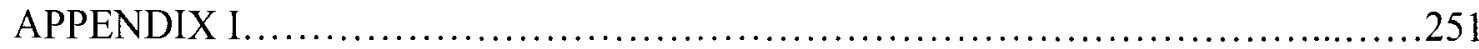

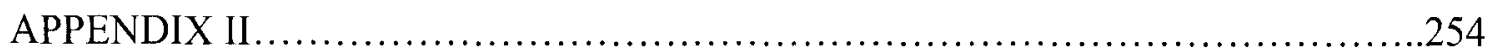

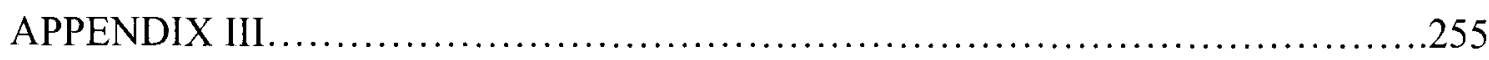

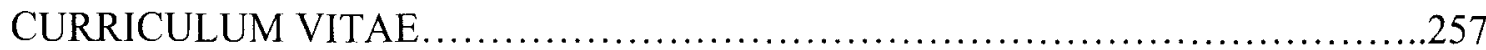




\section{LIST OF TABLES}

TABLE

3.1 Nanomaterials characterization techniques

4.1 Theoretically calculated values for the unit cell parameter, a, and the energy per carbon atom, $\Delta \mathrm{E} / \mathrm{C}$, for various possible $\mathrm{CH}_{\mathrm{x}}$ crystalline phases. .86

5.1 Site-specific surface reactions and the range of rate constants used in the KMC simulations.

7.1 Comparison of the $\Delta \mathrm{Ep}$ values obtained experimentally for different analytes at untreated GC, MCD and NCG electrode materials.

7.2 Deconvoluted C(1s) peak of the XPS spectrum obtained from the NCG electrode material and percentage composition of each functional group.

7.3 Dependence of boundary layer thickness, $\delta$ (at $98 \% \mathrm{C}_{\mathrm{b}}$ ), and $\delta / \mathrm{r}$ on the nanoelectrode radius $(\mathrm{r})$ .203 


\section{LIST OF FIGURES}

FIGURE

PAGE

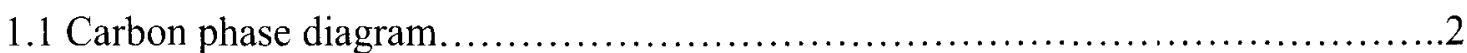

2.1 Structures of proposed precursors for homogenous nucleation of diamond in the gas phase: (a) adamantane, (b) tetracyclodecane, (c) hexacyclopentadecane,

(d) cis boat -boat bicyclodecane, and (e) trans boat-boat bicyclodecane. .10

2.2 Ball and stick model illustrating a mechanism for nucleation of hexagonal diamond by parallel stacking errors resulting from hydrogenation of graphite edge planes......11

2.3 Model of an amorphous carbon cluster (with $\mathrm{C}$ atoms (gray) and $\mathrm{H}$ atoms (white)) derived using density functional theory, with a diamond cell at the center

2.4 Ball and stick model illustrating the mechanism of three-methyl ion step nucleation on diamond $\{111\}$

2.5 Illustrations of different untwinned crystal shapes resulting from the change in $\alpha$-parameter from $\alpha=1$ (cubic), to $\alpha=3$ (octahedral) .21

2.6 Model of two types of stacking of $\mathrm{sp}^{3}$ carbon atoms: (a) staggered-stacking and (b) eclipsed-stacking. These two types of stacking result in cubic and hexagonal-step nucleation of diamond respectively on $\{111\}$ as illustrated in (c).

2.7 SEM images of different morphologies of multiply twinned diamond crystals: 
(a) decahedron, (b) dodecahedron, (c) star-decahedron, (d) star-dodecahedron,

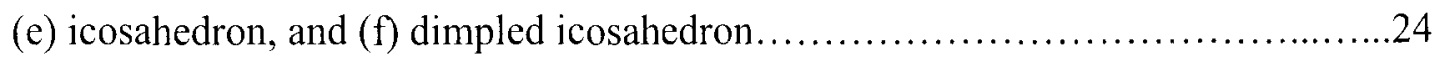

2.8 Ball and stick model of (a) bulk-graphite with basal planes and edge planes,

(b) C60 molecule, and (c) single-walled carbon nanotube....................... 30

2.9 Illustrations of different morphologies of one-dimensional conical carbon nanostructures

3.1 Digital images of (a) 5010 ASTex Microwave plasma CVD reactor and

(b) side-view the quartz chamber with hydrogen plasma (purple glow) above the substrate. .38

3.2 (a) A schematic of multiple platinum wire immersion into a MW plasma. Digital image of (b) graphite substrate stage supported by a steel flange and (c) platinum wires sticking out of the multiple wire-holder.

3.3 A schematic showing various processes that result from electron beam interactions with a specimen

3.4 Illustration of a three-electrode electrochemical cell setup used for cyclic voltammetry. .56

3.5 Equivalent circuit model of an electrochemical cell

4.1 SEM image of the thick carbon deposit at the tip of a platinum wire obtained after deposition at (a) low magnification $(500 \mathrm{X})$ and (b) high magnification (10k X)

4.2 (a) Diffraction pattern of fcc carbon crystals in the [110] zone axis.

(b) Distribution of lattice parameters $\left(a_{0}\right)$ of crystals exhibiting pattern (a) . .68

4.3 (a) Diffraction pattern corresponding to [100] zone axis of a cubic lattice.

Distribution of lattice parameter of crystals exhibiting patterns (a), when indexed as: 
(b) fec and (c) bcc

4.4 (a) Rectangular diffraction pattern of bec carbon crystals in the [110] zone axis.

(b) Distribution of lattice parameters of crystals exhibiting pattern (a).

4.5 (a) Hexagonal diffraction pattern corresponding to lonsdaleite in [001] zone axis or bcc in [111] zone axis. Distribution of lattice parameters of crystals exhibiting patterns (a), when indexed as (b) lonsdaleite and (c) bcc

4.6 (a) The crystal structure of diamond $(\mathrm{Fd} \overline{3} \mathrm{~m})$ and corresponding diffraction pattern in the [110] zone axis.

(b) The crystal structure of fcc $(\mathrm{Fm} \overline{3} \mathrm{~m})$ and corresponding diffraction pattern

in $[110]$ zone axis. .74

4.7 Dynamical diffraction calculations for variation of intensities of spots with diamond crystal thickness in the (a) [110] and (b) [100] zone axis .76

4.8 Model of simulated clusters (a) with $\mathrm{sp}^{3}$ core and $\mathrm{sp}^{2}$ bonds on the surface

(b) bucky-diamond.

4.9 Simulated diamond clusters with (a) large amounts of stacking faults and (b) $30 \%$ vacancy in the structure. .78

4.10 Distribution of the observed ratio of (200) and (111) intensities in the nanodiffraction patterns of $n$-diamond crystals $(a=0.36 \mathrm{~nm})$ in the (a) $[110]$ and (b) $[100]$ zone axis.

4.11 Ball and stick model of a proposed bcc phase of carbon with interpenetrating diamond lattices with a-value of $0.178 \mathrm{~nm}$.

4.12 Proposed structures of (a) $\mathrm{CH}$ fec crystal with a lattice parameter of $0.36 \mathrm{~nm}$ and (b) $\mathrm{CH}_{2}$ bec structure with a lattice parameter of $0.31 \mathrm{~nm}$ and their corresponding 
diffraction patterns in the [100] and [110] zone axis.

4.13 Three-dimensional thermodynamic plot of the free-energy of hydrocarbon species in the gas and solid phases as a function of co-ordination numbers and $\mathrm{H} / \mathrm{C}$ ratios

5.1 SEM images of different multiply twinned diamond crystal morphologies .94

5.2 (a) SEM image of experimentally grown star-shaped diamond crystal.

(b) Indexing different planes and features of the star-shaped crystal....

5.3 SEM images of the sequential growth of a star crystal from (a) $4 \mu \mathrm{m}$ after 3 hours,

(b) $6 \mu \mathrm{m}$ after additional one hour, (c) $8.3 \mu \mathrm{m}$ after additional one hour, and

(d) $12.5 \mu \mathrm{m}$ after additional 1.5 hours of growth 98

5.4 SEM image of (a) star-shaped diamond crystal with no dimple and (b) (a) re-grown for one hour at same conditions with the dimple re-appearing.

5.5 SEM images of (a-b) change in morphology of star-dodecahedral to a stardecahedral with decrease in temperature upon re-growth and (c-d) change in the morphology of a star-decahedral to a dodecahedral with increase in temperature upon re-growth....

5.6 SEM image of $(a-b)$ an intermediate shape crystal re-grown to a completed icosahedron and (c) formation of an additional stacking fault in a star-decahedral crystal, giving rise to an additional dimple

5.7 AFM scan on a star-shaped diamond crystal................................103

5.8 Ball and stick model of Type-1 site, typically found on diamond $\{111\}$ surface ....107

5.9 Ball and stick model of Type- 2 sites on an atom bonded twice to the surface, also known as kink-sites 108 
5.10 Ball and stick model of Type- 3 site found on $\{111\}$ surface, which could give rise to $\{110\}$ type surface after adsorption .108

5.11 Ball and stick model of Type-4 site, which is found on singly-bonded atoms having second neighbors

5.12 Ball and stick model of Type-5 site, which is found on atoms bonded thrice to the surface

5.13 Ball and stick model of Type- 6 site, which is found on an atom bonded twice or thrice to the surface

5.14 Ball and stick model of Type-7 site, which is found on singly or doubly bonded atoms with no first or second neighbors present

5.15 Algorithm for the KMC simulations used in this study

5.16 Ball and stick model of (a) untwinned seed, (b) seed with two stacking errors, and (c) seed with three stacking errors on three different $\{111\}$ planes.

5.17 Wire-frame model of simulated decahedral crystal (5000 atoms) with single adatom adsorption/desorption kinetics $(\mu / \lambda=20)$ (approx. size $6 \mathrm{~nm})$

5.18 Wire-frame model of simulated dodecahedral crystal (5000 atoms) with single adatom adsorption/desorption kinetics $(\mu / \lambda=0.1)$ (approx. size $6 \mathrm{~nm})$

5.19 Wire-frame model of simulated icosahedral crystal (5000 atoms) with single adatom adsorption/desorption kinetics $(\mu / \lambda=20)$ (approx. size $4 \mathrm{~nm}$ ).

5.20 (a) Wire-frame and (b) space-filled model of a simulated star-dodecahedral with single adatom adsorption/desorption and doubly bonded atom etching $(\lambda / \mu=0.4$, $\left.\mu_{2}=0.2\right)$ (approx. size $5 \mathrm{~nm}$ ).

5.21 (a) Wire-frame and (b) space-filled model of a simulated star-decahedral crystal 
containing 7000 atoms with a prominent dimple using rate constants $\mu_{1}=5, \mu_{4}=5$, $\lambda_{2}=10, \lambda_{3}=1, \lambda_{4}=1, \lambda_{5}=1, \lambda_{6}=5, \lambda_{7}=1$ (approx. size $6 \mathrm{~nm}$ )

5.22 (a) Wire-frame and (b) space-filled model of a long-term simulation of a star-decahedral crystal with 19500 atoms (approx. size $10 \mathrm{~nm}$ ).

5.23 Space-filled model of a simulated growth sequence of a star-decahedral at

(a) 7000 atoms with a prominent dimple and (b) 7500 atoms with dimple partially filled 131

5.24 (a) Wire-frame model and (b) space-filled model of a simulated decahedral crystal containing 7000 atoms using rate constants $\mu_{1}=10, \mu_{4}=10, \lambda_{2}=5, \lambda_{3}=10, \lambda_{4}=1, \lambda_{5}=1$, $\lambda_{6}=10, \lambda_{7}=1$ (approx. size $6 \mathrm{~nm}$ ).

5.25 Wire-frame model of a simulated dodecahedral crystal containing 7000 atoms using rate constants $\mu_{1}=2, \mu_{4}=2, \lambda_{2}=2, \lambda_{3}=10, \lambda_{4}=2, \lambda_{5}=1, \lambda_{6}=1, \lambda_{7}=2$ (approx. size $5 \mathrm{~nm})$

5.26 Space-filled model of (a) simulated pefect-icosahedaral (same rate constants as decahedral crystals) and (b) simulated dimpled icosahedron (same rate constants as star-decahedral).

5.27 Schematic side-view of either a decahedral or star-decahedral crystal.... 136

5.28 Logarithmic plot of number of atoms vs. time (arbitrary units) for (a) simulated star-decahedral crystal and (b) simulated decahedral crystal.

5.29 Simulated step propagation sequences on a star-decahedral crystal at different sizes:

(a) 1900 atoms and (b) 2000 atoms. Wire-frame model illustrating the

bonding nature of the step

5.30 Simulated step propagation sequences on a decahedral crystal at different sizes: 
(a) 1700 atoms and (b) 1800 atoms. Wire-frame model illustrating the bonding nature of the step.

5.31 Ball and stick model indicating the two primary nucleation sites on a stardecahedral crystal: type-3 at the twin boundary and type-4 on a $\{111\}$ plane

5.32 Wire-frame model indicating the types of doubly bonded atoms that would be etched in simulations where $\mu_{2} \neq 0$. 144

6.1 SEM image of carbon nanopipettes emerging from the microcrystalline diamond film on a platinum wire.

6.2 SEM image showing a large density of randomly oriented carbon nanopipettes.

6.3 (a-c) SEM images indicating surface faceting of the nanopipettes, and appearance of rolled-up sheets

6.4 Raman spectra taken with laser wavelength $785 \mathrm{~nm}$ from (a) microcrystalline diamond film and (b) nanopipettes emerging from the diamond film

6.5 Bright field TEM image of the nanopipettes, indicating a hollow core. 153

6.6 Dark field TEM image taken from the 00.4 Bragg reflection, and inset indicating the corresponding EFTEM image from the tip of the nanopipette using $\mathrm{sp}^{2}$ carbon core loss peak

6.7 (a) Diffraction patterns from carbon nanopipettes indexed as basal plane of graphite.

(b) Diffraction pattern outlining the helical sheets with angle $\sim 9^{\circ}$

6.8 (a) Dark field image of a broken nanopipette, indicating a protruding central nanotube, which has the same brightness as the shell of the pipette.

(b) Diffraction pattern from the broken nanopipette corresponding to helical sheet of graphite. .156 
6.9 Schematic of the overall structure of the carbon nanopipette

6.10 Varying aspect ratio structures along the length of the platinum wire:

(a) conical structures with a central nanotube, (b) increased aspect ratio of conical structures, and (c) carbon nanopipettes.

6.11 Growth mechanism of carbon nanopipettes: (a) growth begins with an initial nanotube, (b) wrapping-up of helical sheets of graphite around the central nanotube

(c) simultaneous growth and etching results in high aspect ratio pipettes. 162

6.12 SEM image of a $75 \mu \mathrm{m}$ long carbon nanopipette.

6.13 SEM image of carbon nanopipette array on a platinum sheet substrate.

6.14 SEM images of (a) carbon nanostructures grown on gold wire-substrate and

(b) graphite deposit growing around the nanostructures

7.1 (a) SEM image of the ball-shaped deposit on the platinum wire (inset shows the overall tip).

(b) EDS of the ball-shaped deposit

7.2 XRD spectrum of the scraped ball-shaped deposit

7.3 Micro-Raman spectra taken from the ball-shaped nanocomposite deposit using different laser excitation wavelengths of $785 \mathrm{~nm}, 514.5 \mathrm{~nm}$, and $442 \mathrm{~nm}$. The inset shows the dependence of D-band wavelength on the laser energy, with a slope of $50 \mathrm{~cm}^{-1} / \mathrm{eV}$

7.4 Raman spectra with calculated integrated intensity ratio for $\mathrm{G} / \mathrm{D}$ band $(\mathrm{R})$ for laser wavelengths of (a) $785 \mathrm{~nm}$ (b) $633 \mathrm{~nm}$ 175

7.5 Dark field image taken from the intact bulb-part of the electrode. Corresponding 
electron nanodiffraction patterns indicated in the insets are from $1 \mathrm{~nm}$ region.

7.6 $\mathrm{CV}$ in $0.5 \mathrm{M}$ sulfuric acid solution at $100 \mathrm{mV} / \mathrm{s}$ scan rate at the (a) NCG, (b) MCD, and (c) GC electrode.

7.7 CV of $1 \mathrm{mM} \mathrm{K}_{3} \mathrm{Fe}(\mathrm{CN})_{6}$ in $0.1 \mathrm{M} \mathrm{KCl}$ solution at scan rates from $20 \mathrm{mV} / \mathrm{s}$ to 200 $\mathrm{mV} / \mathrm{s}$ at NCG. Inset shows that the variation of cathodic current with scan rate ${ }^{\wedge} 0.5$ is linear 182

7.8 CV of $1 \mathrm{mM}$ (a) 4-Methyl catechol, (b) ruthenium hexamine hydrochloride,

(c) catechol, (d) ferricyanide, (e) hydroquinone, and (f) dopamine in $0.1 \mathrm{M}$

$\mathrm{KCl}$ solution at $100 \mathrm{mV} / \mathrm{s}$ scan rate at $\mathrm{NCG}$ electrode. 185

7.9 CV of $1 \mathrm{mM}$ dopamine in $0.1 \mathrm{M} \mathrm{KCl}$ solution at (a) NCG, (b) Pt, (c) GC, and (d) MCD different electrodes at $100 \mathrm{mV} / \mathrm{s}$ scan rate 188

7.10 $\mathrm{CV}$ of $1 \mathrm{mM}$ dopamine in $0.1 \mathrm{M} \mathrm{KCl}$ at different scan rates at $\mathrm{NCG}$ electrode. Inset shows that the variation of anodic current with scan rate ${ }^{\wedge} 0.5$ is linear. 189

$7.11 \mathrm{CV}$ of $1 \mathrm{mM}$ dopamine in $0.1 \mathrm{M} \mathrm{KCl}$ at $100 \mathrm{mV} / \mathrm{s}$ scan rate at $\mathrm{NCG}$ electrode before and after acid treatment.

7.12 XPS spectra for C(1s) taken on the (a) as-synthesized well-working NCG electrode, (b) as-synthesized NCG electrode with irreversible behavior, and (c) after anodic oxidation of (b) in $0.5 \mathrm{M} \mathrm{H}_{2} \mathrm{SO}_{4}$ at $2.0 \mathrm{~V}$.

7.13 Bode plots in $0.1 \mathrm{M} \mathrm{KCl}$ solution showing the effect of anodic oxidation on the double layer capacitance of NCG electrode. 
7.14 CV of $1 \mathrm{mM}$ dopamine in $0.1 \mathrm{M} \mathrm{KCl}$ at $100 \mathrm{mV} / \mathrm{s}$ scan rate repeatedly on the same NCG electrode over a period of 1 month 196

7.15 CV of a mixture of $2 \mathrm{mM}$ of ascorbic acid, $10 \mu \mathrm{M}$ dopamine, $10 \mu \mathrm{M}$ serotonin in $0.1 \mathrm{M} \mathrm{KCl}$ solution at $100 \mathrm{mV} / \mathrm{s}$ at $\mathrm{NCG}$ electrode.

7.16 CV obtained after anodic oxidation during continuous scanning (100 scans) at $100 \mathrm{mV} / \mathrm{s}$ at $\mathrm{pH} 7$ phosphate buffer solution containing (a) mixture of $10 \mathrm{mM}$ of ascorbic acid, $0.1 \mathrm{mM}$ dopamine mixture (b) $10 \mathrm{mM}$ ascorbic acid at

NCG electrode. 199

7.17 Dependence of the boundary layer thickness, $\delta$, with spherical nanoelectrode radius. .203

7.18 (a) SEM image of randomly oriented pipettes on a platinum wire.

(b) Steady-state response from the pipettes to a $1 \mathrm{mM}$ dopamine in $0.1 \mathrm{M} \mathrm{KCl}$.

(c) Dependence of anodic current on scan rate. .206

7.19 (a) SEM image of array electrode containing cones with a central nanotube.

(b) SEM image of nanopipettes about $2 \mathrm{~mm}$ away from the tip. 208

7.20 (a) $\mathrm{CV}$ of $1 \mathrm{mM}$ dopamine in $0.1 \mathrm{M} \mathrm{KCl}$ at $100 \mathrm{mV} / \mathrm{s}$ before and after electrochemical treatment in $0.5 \mathrm{M} \mathrm{H}_{2} \mathrm{SO}_{4}$ at the nanopipette array electrode. (b) $\mathrm{CV}$ of $1 \mathrm{mM} \mathrm{K}{ }_{3} \mathrm{Fe}(\mathrm{CN})_{6}$ in $0.1 \mathrm{M} \mathrm{KCl}$ at $100 \mathrm{mV} / \mathrm{s}$ to $600 \mathrm{mV} / \mathrm{s}$. Inset shows dependence of cathodic current with scan rate $(\mathrm{V} / \mathrm{s}) \wedge 0.5$ .209

7.21 Schematic of a closely spaced array of nanopipettes (a) without coating and (b) with polymer coating .211 
7.22 SEM images of (a) an uncoated array of nanopipettes, sequential coating of the array with enamel (b) after $1^{\text {st }}$ dip-coating, and (c) after second dip-coating...212

9.1 SEM images of star-shaped morphologies in other crystalline materials like

(a) gallium nitride (cubic or hexagonal crystal), (b) hexagonal boron nitride, and

(c) hexagonal gibbsite crystals 220

9.2 Schematic of the proposed smart drug-delivery patch using an array of carbon nanopipettes .222

A1.1 Ball and stick model of the diamond $\{100\}$ plane .251

A1.2 Ball and stick model of the two types of $\{111\}$ planes with atoms bonded

(a) thrice to the surface (b) with atoms bonded only once to the surface .252

A1.3 Ball and stick model of diamond $\{110\}$ plane 253

A2.1 (a-d) Wire-frame model of a simulated sequence of five-fold twinned crystals converting to an icosahedral crystal by the addition of a stacking fault during growth.

A 3.1 Space-filled model of the initial stages of decahedron growth till 1000 atoms....255

A 3.2 Space-filled model of the initial stages of star-decahedron growth till 1000 atoms .256 


\section{CHAPTER 1}

\section{INTRODUCTION}

Till the mid-1980s, only two primary phases of carbon were known to exist, diamond and graphite. The discovery of fullerenes (C60 and C70) in 1985 [1] initiated the research efforts on carbon nanotubes and their varied morphological manifestations.

The phase diagram for carbon adapted from references 2 and 3 is shown in Figure 1.1. This figure indicates the thermodynamically stable regimes for the existence of diamond, graphite and liquid carbon. Once a carbon phase is formed, the atoms are locked into the given phase configuration, and a large activation energy is required to convert it to another thermodynamically stable phase. Thus, a number of carbon polymorphs can typically exist in metastable states well into a region (of temperature and pressure) where a different carbon solid phase is thermodynamically stable. One of the best examples is diamond, which at room temperature and pressure is thermodynamically unstable with respect to graphite by $2.9 \mathrm{~kJ} / \mathrm{g}$. At the same time, the transformation of diamond to graphite is kinetically unfavorable. Similarly, the possibility of discovering new/metastable phases of carbon and studying their unique properties and applications will continue to instigate scientific curiosity.

Carbon exists in two preferred hybridization states for the known crystalline phases: planar $\mathrm{sp}^{2}$ found in graphite and tetrahedral $\mathrm{sp}^{3}$ found in diamond. Diamond's gemological importance dates back to the early history of mankind. However its 
exceptional mechanical, thermal and semiconducting properties were realized much later. These superior properties of diamond can be harnessed into engineering applications only when diamond can be made in various forms such as powders/abrasives, thin and thick polycrystalline films, and large area single-crystals or single-crystal quality films.

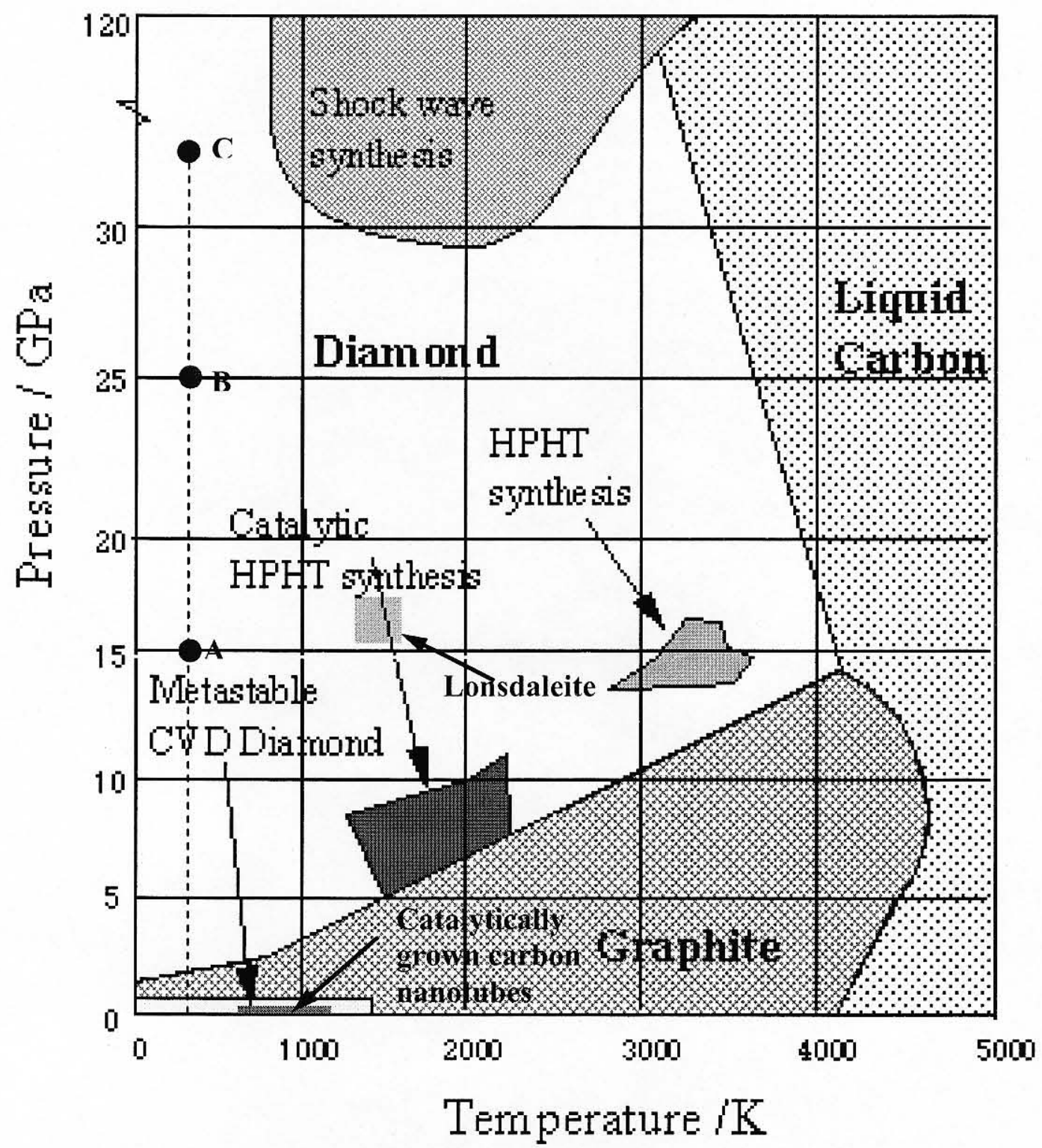

Figure 1.1 Carbon phase diagram, adapted from ref. 2, 3. Line A-B-C indicates the path along which graphite, when compressed along the c-axis at room temperature, acquires properties consistent with diamond-like polytypes. 
The first man made diamond was synthesized under thermodynamically stable conditions, i.e. high-pressure high-temperature (HPHT) conversion of graphite to diamond (refer to Figure 1.1) $[4,5]$. Due to the harsh process conditions, the HPHT process is not practiced for many applications, except for abrasives. In the 1960s, contrary to thermodynamic predictions, a breakthrough was made in the synthesis of diamond by low pressure chemical vapor deposition (CVD) $[6,7,8]$. CVD is now used routinely for growing polycrystalline diamond films. To realize the theoretically predicted properties of diamond, textured-films of diamond are required, which can be achieved only by growing thick films $(>100 \mu \mathrm{m})[9]$. This requires high nucleation densities followed by high growth rates. Nanocrystalline diamond films, a recent development largely used for micro electromechanical systems, also require increased nucleation densities $[10,11]$. Thus, increased nucleation and growth rates are key aspects for growing thin/thick films of diamond from the vapor phase.

Heteroepitaxy onto single-crystal non-diamond substrates could possibly produce single crystal quality thin films of diamond. However, due to the lattice mismatch with many available single crystal substrates, heteroepitaxy is quite challenging as it leads to discontinuous and defective layers. Some of the substrates that have shown limited success with obtaining few layers of diamond on limited areas are silicon, silicon carbide, nickel, cubic boron nitride and iridium [12]. Iridium is the best-known substrate giving the lowest angle spread of diamond-crystal orientation [13]. The limitation, however, is that free-standing single-crystal iridium wafers are not yet available. Hence, the availability of the most suitable substrates with minimum lattice mismatch for the heteroepitaxial growth of diamond wafers still remains a challenge. 
Recently, Apollo Diamond Inc. patented a technique for synthesizing gem-quality diamond (several $\mathrm{mm}$ in size) homoepitaxially on pre-existing diamond crystals (possibly natural diamond or HPHT diamond) $[14,15]$. Defect control during homoepitaxy is very important. Therefore, the technique patented by Apollo uses stress relaxation of the lattice by alternating boron-doped with undoped layers, thus controlling the defects in the homoepitaxially grown diamond layers. However, the lack of large area single-crystal diamond substrates makes it difficult to produce diamond wafers by homoepitaxy.

Through all these attempts at diamond synthesis, it was clear that the growth of defect free single-crystals and thin films from vapor phase requires a clear understanding of nucleation and growth mechanisms of individual crystals. Although there have been several hypotheses in literature, there is no experimental evidence on the structure of diamond nuclei. In addition, a number of reports claimed the existence of new phases of carbon at the nanoscale synthesized under diamond growing conditions by CVD and HPHT, called n-diamond and i-carbon [16-28]. Their structural analysis suggested hybridization states different from $\mathrm{sp}^{2}$ or $\mathrm{sp}^{3}$ carbon, making it difficult to understand their crystallographic structure.

Similarly, nanocrystalline morphologies of graphite are known to be more diverse. A number of forms of graphite still seem to be unexplored, due to the lack of systematic growth studies. Graphite is an anisotropic material with highly reactive edge planes and inert basal planes. Fullerenes and carbon nanotubes are nanocrystals obtained by simply rolling up graphene sheets. It is not clear yet whether graphite crystals at the nanoscale can still retain their bulk structure. The minimum size above which these nanocrystals of graphite would tend to transform into other phases in the presence of 
hydrogen is also unknown. If nanocrystalline graphite existed, it would have a very high electronic density of states due to the large fraction of edge planes and could have interesting applications [29].

Thus, the knowledge of the structure of carbon crystals at the nanoscale obtained under diamond growing conditions could yield insight into various phases for carbon and possibly provide clues into diamond nucleation.

The growth of crystals at small length scales (early stages) could also provide the route to understand the chemistry behind the formation of diamond. For example, diamond deposition under typical growth conditions always results in decahedrons, stardecahedrons, dodecahedrons, and twenty-fold icosahedrons along with perfect diamond crystals. Morphological control of these individual crystals is important in growing oriented or textured polycrystalline diamond films, as well as large-size, defect-free diamond single crystals [30]. This can be accomplished by understanding the kinetics of the reactions that control the faceting of crystals at early stages. Additionally, inadequate knowledge of the detailed gas-surface reactions makes it necessary to use theoretical methods for predicting kinetics of diamond growth. Kinetic Monte Carlo (KMC) simulations provide an efficient means of simulating individual crystal growth at physical sizes observed in experiments and testing various assumptions on the molecular-level processes (gas-surface reactions). By comparing the simulated crystals to the experimentally grown ones, the kinetic mechanisms responsible for their morphologies can be determined.

Currently, there exists no method to directly observe nucleation and growth of crystals from vapor phase. Thus, this dissertation is focused on understanding the 
structures of nanocrystalline phases of carbon and the kinetic mechanisms that control their morphology using the following experimental and theoretical tools.

$>\quad$ Investigating the structure of carbon crystals at the nanoscale could yield information on structures of metastable phases and their relationship to mucleation of diamond and graphite and possibly lead to the discovery of new phases of material.

$>\quad$ Studies on the kinetic faceting at early stages of growth to provide clear understanding on the growth mechanisms involved in defects and morphologies of micro/nanocrystals.

This dissertation is divided into nine main chapters. Chapter 2 provides an overview of the extensive literature on different crystalline structures and the morphological manifestations of diamond and graphite. Chapter 2 also elucidates several challenges in understanding the nucleation, crystal structure of metastable phases and morphological evolution of diamond and specific forms of $\mathrm{sp}^{2}$ carbon.

Chapter 3 provides details of the experimental techniques employed for capturing nanocrystals and characterization tools used to analyze their structure and morphology.

Chapter 4 elucidates the nanodiffraction studies performed on the structures of cubic carbon nanocrystals synthesized by CVD. Diffraction analysis indicated a new body-centered cubic (bcc) phase of carbon along with some known metastable phases of face-centered-cubic carbon, n-diamond and i-carbon.

Chapter 5 describes the Kinetic Monte Carlo (KMC) simulations of individual crystal growth using various sets of detailed surface chemistry. Chapter 5 also describes 
interrupted growth experiments for understanding morphological evolution. In addition, the morphologies of simulated crystals are compared with the experimentally grown ones to link the molecular level and process level understanding.

Chapters 6 and 7 describe new morphological forms of $\mathrm{sp}^{2}$ carbon in the form of nanocrystalline graphite and carbon nanopipettes discovered as a part of this work. Chapter 6 describes the morphology and structural characterization of carbon nanopipettes. Chapter 7 describes the electrochemical investigations using nanocrystalline graphite and carbon nanopipettes for sensing biologically important amines and catechols. Chapter 7 also describes the development of a nanoarray electroanalytical sensor based on nanopipettes.

Chapters 8 and 9 provide conclusions and discussions on the broad impacts of the results on carbon based crystalline materials and point to the future scope of this work. 


\section{CHAPTER 2}

\section{BACKGROUND}

\subsection{Introduction}

In this chapter, an overview of the current understanding of the nucleation, chemistry of growth, and morphological evolution of diamond films/crystals synthesized from the vapor phase is presented. The morphologies of different nanocrystalline forms of graphite synthesized from vapor phase are also reviewed. This review intentionally does not include some of the extensive literature available on diamond synthesis (e.g. high pressure synthesis, polycrystalline films, effect of dopants, etc.) [31-34] and much of the carbon nanotube literature (e.g. single-walled, multi-walled nanotube synthesis) [3537]. The reader is referred to the suggested review articles for further information.

\subsection{Low pressure synthesis of diamond from the vapor phase}

Low-pressure chemical vapor deposition (CVD) is an attractive and cost effective method of synthesizing diamond. Using a hydrocarbon source diluted in hydrogen with gas phase activation, diamond is deposited onto a pre-existing template (pre-existing diamond seeds or by homoepitaxy on diamond or by heteroepitaxy). The first reactor used for diamond CVD was a hot-filament reactor, following which microwave plasma 
CVD systems were extensively used. Some of the pioneering work on diamond CVD can be found in references $[7,8,38,39]$. The different forms of gas phase activation that are commonly used now are thermal methods (hot filament), electric discharge (DC, RF or microwave) or combustion flame (oxyacetylene torch). For more details on reactor systems, the reader may also refer to several excellent review articles on diamond CVD and the references therein $[31,32,40-43]$.

Though different types of reactors based on different gas phase activations are available, the typical conditions used for diamond synthesis are $1-2 \%$ of methane in hydrogen and substrate temperatures of around $700{ }^{\circ} \mathrm{C}$. Irrespective of the activation method, for the range of feed gas compositions that can be used, there exists only a narrow window where diamond deposition occurs. Bachman et al. devised an empirical ternary diagram based on feed gas compositions to aid the selection of process conditions that would result in diamond deposition [44]. To further the use of this diagram, a C-H-O ternary diagram was developed to predict diamond deposition based on the concentration of radical species in the gas phase $[45,46]$. However, these diagrams are not useful in understanding the nucleation on the substrate from the vapor phase. Since diamond is a metastable phase and nucleates from the gas phase heterogeneously, the classical nucleation theory for predicting the critical nuclei diameter based on carbon supersaturation is not really applicable [47]. Thus, over the years, several researchers have hypothesized structures and kinetic pathways that lead to nucleation.

As early as 1983, Matsumoto and Matsui [48] suggested that hydrocarbon cage molecules such as adamantane, tetracyclodecane and hexacyclopentadecane could be the gas phase molecular precursor for the nucleation of diamond. Adamantane $\left(\mathrm{C}_{10} \mathrm{H}_{16}\right)($ See 
Figure 2.1) represents the smallest combination of carbon atoms with a perfect diamond structure. The latter two are twinned diamond embryos that were proposed as precursors to fivefold twinned diamond crystals. However, thermodynamic arguments suggested that cage compounds such as adamantane would be highly unstable in diamond growth environments, where the temperature can exceed $1000^{\circ} \mathrm{C}$ [49]. Later, fully hydrogenated ring compounds like bicyclodecane were argued as more plausible precursors due to their abundance in diamond growing environments and their kinetically preferred sites for adatom addition [50]. This idea also had similar thermodynamic and kinetic limitations.

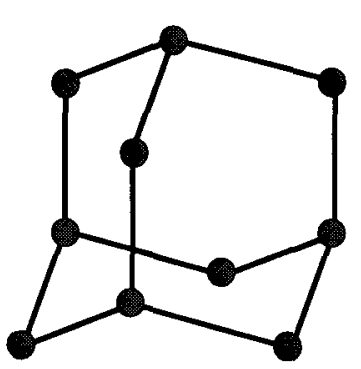

(a)

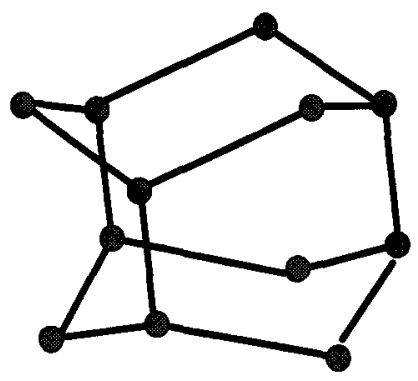

(b)

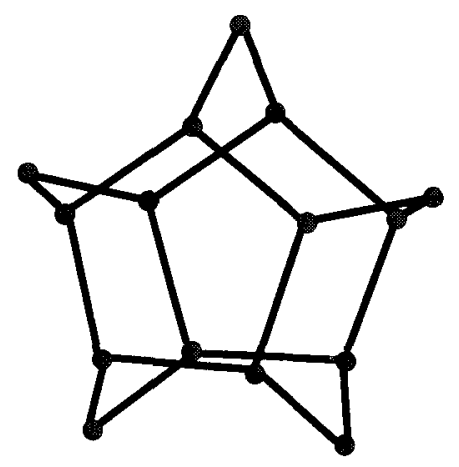

(c)
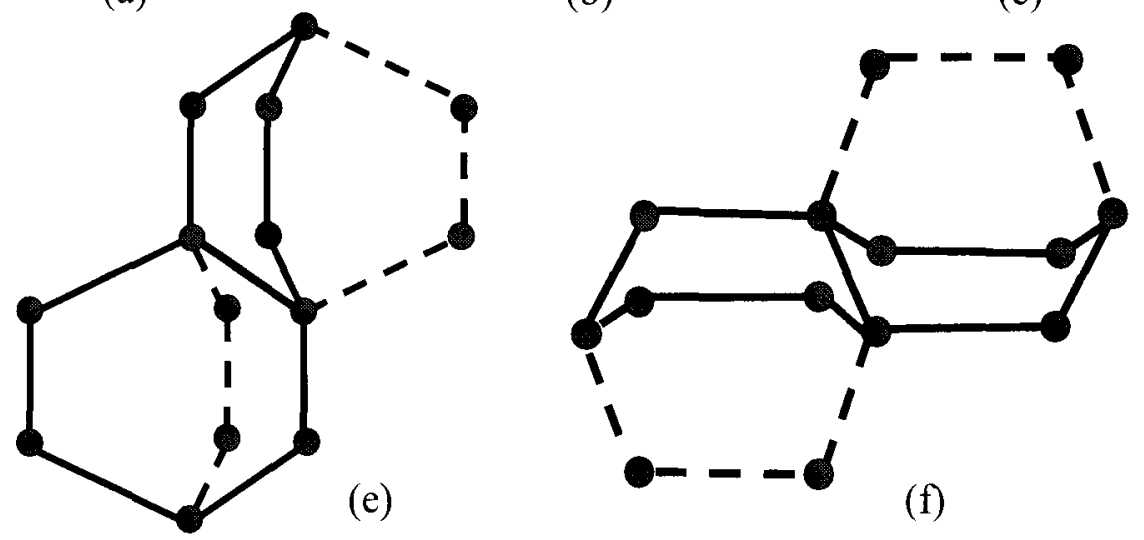

(f)

Figure 2.1 Structures of proposed precursors for homogenous nucleation of diamond in the gas phase: (a) adamantane, (b) tetracyclodecane, (c) hexacyclopentadecane, (d) cis boat-boat bicyclodecane, and (e) trans boat-boat bicyclodecane [48]. 
The role of hydrogen in the stability of diamond nanocrystals was shown by Badziag et al. by comparison of the heats of formation of tetrahedral clusters (diamond) terminated with hydrogen and hexagonal (graphitic) clusters [51]. Angus et al. [52] suggested that hydrogenation of graphitic edges could be the likely route to form diamond nuclei. With this hypothesis, the presence of parallel stacking errors is required for faster growth in the early stages to form stable nuclei. This early stage growth would lead to diamond nuclei with predominantly hexagonal structure (see Figure 2.2).

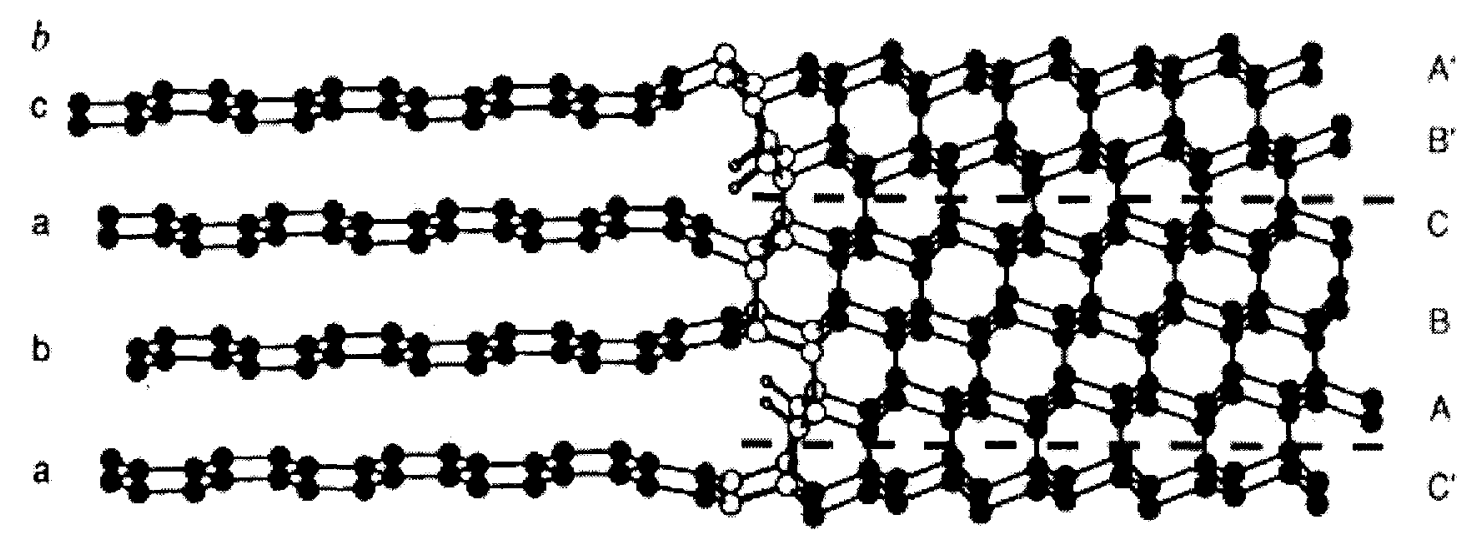

Figure 2.2 Ball and stick model illustrating a mechanism for nucleation of hexagonal diamond by parallel stacking errors resulting from hydrogenation of graphite edge planes. (Courtesy ref. [52]).

Recently, a few attempts were made to study the size and structure of the smallest diamond nanocrystal [53]. Studies using heteroexpitaxy on Si substrates revealed that the smallest heteroepitaxial nuclei $(\sim 2 \mathrm{~nm})$ were defect-free. This is not surprising, as one would not expect any defects arising from lattice mismatch during epitaxy at such small sizes. Attempts to study diamond crystals grown non-epitaxially were not successful, as 
they were limited to large sizes $(\sim 1 \mu \mathrm{m})$ and so did not provide enough insight into the nuclei structure [54].

Another mechanism was proposed for bias-enhanced nucleation of diamond on silicon substrates [55]. This mechanism, supported by molecular dynamics simulations, involved formation of an amorphous-C:H phase in subsurface layers of the substrate (silicon). This study primarily suggested that diamond nucleation occurred within the bulk of amorphous carbon layers (see Figure 2.3). Thus, there is a need for direct determination of diamond nucleation occurring from the vapor phase.

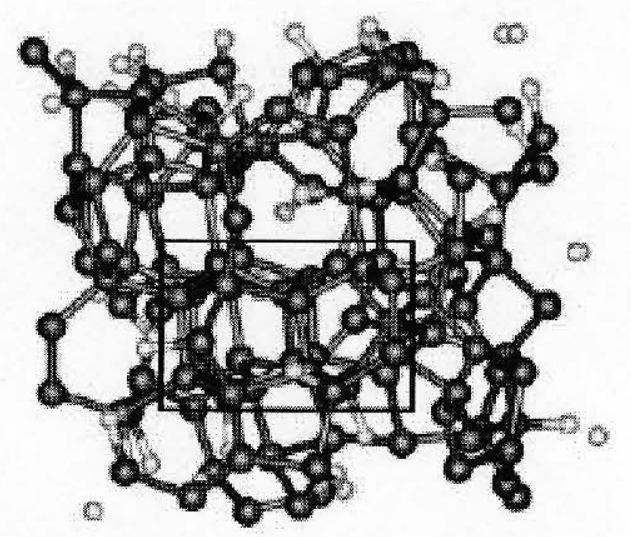

Figure 2.3 Model of an amorphous carbon cluster (with $\mathrm{C}$ atoms (gray) and $\mathrm{H}$ atoms (white)) derived using density functional theory, with a diamond cell at the center (courtesy Ref. 55).

All of the suggested models to date lack experimental evidence for the confirmation of structures of the nuclei or smallest nanocrystals formed during synthesis. This is because it is difficult to capture a considerable number of nuclei at sizes lesser than a few $\mathrm{nm}$ and study their individual structures. Thus, an experimental procedure for 
capturing a large density of diamond nuclei is proposed in this work, for studying their structures using a special technique of electron nanodiffraction in a transmission electron microscope (TEM).

\subsection{Other polymorphs and unusual phases of crystalline carbon}

Several polymorphs of carbon are known to exist in metastable forms. Lonsdaleite (or hexagonal diamond) is one of the well-known metastable forms, which has $\mathrm{sp}^{3}$ bonding similar to diamond but with a different layering sequence [56]. When visualized in the [111] direction, lonsdaleite has an $\mathrm{ABAB}$ stacking, unlike diamond that has an $\mathrm{ABCABC}$ stacking (see Figure 2.2). The lattice parameters of lonsdaleite are $\mathrm{a}=$ $0.252 \mathrm{~nm}$ and $\mathrm{c}=0.512 \mathrm{~nm}$ compared with diamond which has $\mathrm{a}=0.356 \mathrm{~nm}$. Several researchers reported experimental evidence for lonsdaleite during the synthesis of diamond $[50,57]$. This phase was also linked to nucleation of diamond from graphitic precursors [52]. Similarly, at high temperatures and pressures, several other polymorphs of carbon such as carbynes, chaoite and carbon VI may also be produced [16].

Other than the above polymorphs, two new phases of carbon, which have cubic crystal structures different from diamond, were reported by several researchers. i-carbon had a crystal structure similar to diamond, but with a lattice parameter significantly different, and showed the (200) and (222) reflections (spots) in the electron diffraction patterns in one report [17] and (110) and (211) reflections in another [18]. These reflections are forbidden for the usual cubic diamond phase. i-carbon was synthesized by decomposition of hydrocarbon gases in a radio-frequency plasma and using the ion-beam technique $[17,18]$. The lattice parameters reported for $\mathrm{i}$-carbon phase were $0.425-0.445$ 
nm. The second new phase, called "n-diamond", had a lattice parameter same as diamond (3.56 A), but the diffraction pattern had the (002), (222), (420) reflections that are normally forbidden for diamond. Thus, the n-diamond and i-carbon were both fcc cubic phases. n-diamond was initially synthesized by rapid-cooling of graphite sheets shock-compressed to very high temperatures and pressures $[19,20]$. Later, the $n-$ diamond phase was observed in several other techniques: homogenous nucleation of diamond directly from the gas phase [50], synthesis of diamond from silicon carbide [21], Ru-doped nanostructured carbon films [22], carbon ion-implantation into quartz [23], and hydrogen-plasma etching of CVD grown diamond films [24, 25]. Incidentally, in all these methods, perfect diamond-cubic nanocrystals were also found along with ndiamond nanocrystals. In all of the above methods, the lattice parameter for n-diamond was consistently close to that of diamond $(0.356 \mathrm{~nm})$, and the intensity of the forbidden (002) reflection was quite significant in comparison to the (111) reflection. However, a few reports presented evidence for diffraction patterns similar to n-diamond, but with larger lattice parameters. These reports claimed n-diamond crystals with lattice parameters $0.545 \mathrm{~nm}$ were synthesized from soot [26] and graphite $[27,28]$ under high temperature and pressures, respectively.

Initial theoretical analysis based on local density approximation [24] suggested that, for fcc carbon structure, the cell dimensions for maximum stability should be about $0.356 \mathrm{~nm}$ as observed experimentally in the different reports. But later, more complete calculations [58] suggested that the most stable cell dimension would be $0.308 \mathrm{~nm}$. Murrieta et al. [59] found that the total energy shows a minimum for isotropic deformation at $\mathrm{a}=0.308 \mathrm{~nm}$, but the total energy exhibits a maximum for tetragonal and 
trigonal deformations. They concluded that the fcc structure is not a true metastable phase, but may possibly be stabilized by influence of a substrate or by impurities. This substrate/impurity stabilization may increase the lattice parameter to $0.356 \mathrm{~nm}$, as was commonly observed experimentally.

Electron energy loss spectroscopy (EELS) was carried out on the n-diamond samples to confirm the absence of any chemical contamination [24]. In fact, EELS for ndiamond showed a characteristic edge offset distinguishing it from regular diamond, amorphous carbon, and graphite. Transmission electron microscope (TEM) - Energy dispersive $\mathrm{X}$-ray spectroscopy (EDX) analysis also did not indicate the presence of any other material within its detection limits [28].

In all the studies discussed above, the n-diamond and i-carbon phases were reported for crystal sizes lesser than $100 \mathrm{~nm}$, irrespective of the methods used for synthesis. This may suggest that these new phases may be intermediate forms towards the formation of diamond. In addition, based on theoretical calculations, these phases were also shown to have properties different from bulk diamond. For instance, hexagonal diamond has a higher bulk modulus compared to diamond [60] and hence could surpass diamond in hardness, and n-diamond could be metallic in character, compared to diamond which is electrically insulating [24]. However, the structure of carbon in the fcc crystalline form is not yet known.

A thorough investigation is necessary to understand the structure and occurrence of these new phases of carbon. The outcomes of these investigations could provide an insight into the intermediate phases involved in diamond nucleation or could lead to possible discovery of new metastable phases. Thus, a detailed study of the structures of 
nanosized diamond crystals is proposed using electron nanodiffraction and some theoretical tools for corresponding analysis.

\subsection{Chemistry of diamond growth from the vapor phase}

There are several complex chemical and physical processes that occur during diamond CVD. Gas phase activation (e.g. a hot filament or microwaves) is used to create reactive fragments from the reactant gases (hydrogen and hydrocarbons) in addition to providing heat. These reactive fragments undergo gas phase recombination by collisions, and ultimately strike the surface, where they adsorb and react, or may aid in desorbing existing surface species. The ultimate result of these processes is diamond growth. Diamond growth is dependent on the gas phase reactants, temperature, pressure, type of gas phase activation as well as the reactor.

There were several suggestions for the diamond growth species at the gas-solid interface, including $\mathrm{C}, \mathrm{CH}, \mathrm{C}_{2}, \mathrm{C}_{2} \mathrm{H}, \mathrm{CH}_{3}, \mathrm{C}_{2} \mathrm{H}_{2}, \mathrm{CH}_{3}{ }^{+}$, etc $[32,41,43]$. Such studies have involved theoretical as well as experimental confirmations.

One of the earliest reports [61] on diamond growth chemistry used quantum mechanics to determine the unique reaction route that leads to diamond growth. Using $\mathrm{CH}_{3}{ }^{+}$ionic species, the mechanism proposed was that methyl groups first cover the $\{111\}$ plane of diamond surface, possibly by hydrogen abstraction. Then three neighboring methyl groups are bound spontaneously in one step by a single methyl cation. This forms a stable step on the diamond $\{111\}$ surface (See Figure 2.4). The epitaxial growth of the $\{111\}$ occurs when the same chain of reactions repeats. 


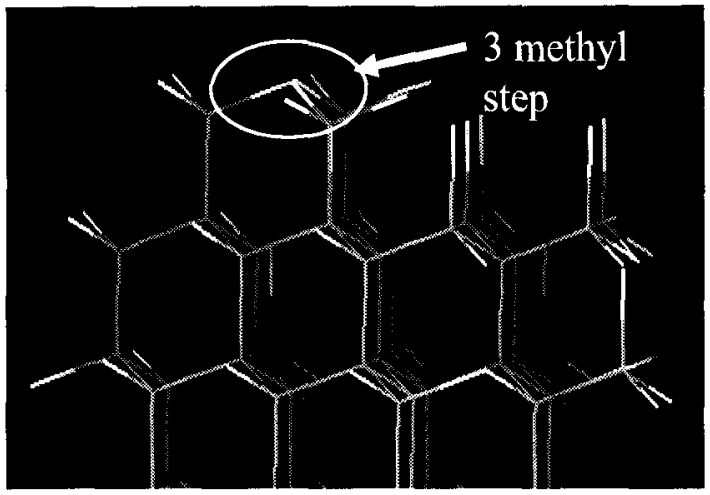

Figure 2.4 Ball and stick model illustrating the mechanism of three-methyl ion step nucleation on diamond $\{111\}[61]$.

The possibility of this mechanism was discounted on the basis that stearic repulsions can exist between the three hydrogen atoms of methyl radicals, due to which the methyl adatoms can be easily desorbed from the surface [62]. Also, the fact that diamond growth could occur in environments with no charged species (like a hot filament reactor) discounted the possibility of ionic species being responsible for diamond growth.

Using just methyl radicals for simulating diamond growth, the predicted growth rate could not account for the experimentally observed growth rate on diamond $\{111\}$ [63]. Using semi-empirical quantum mechanical simulations, a mechanism involving $\mathrm{H}$ abstraction and acetylene addition was proposed for propagation of diamond growth epitaxially $[63,64]$. The adsorbed acetylene is attached to the diamond surface by a single C-C bond. To form a stable step (or hexagonal ring of $\mathrm{sp}^{3}$ carbon), the addition of a second $\mathrm{C}_{2} \mathrm{H}_{2}$ is necessary [65]. However, desorption of $\mathrm{C}_{2} \mathrm{H}_{2}$ occurs quickly from the surface compared to $\mathrm{CH}_{3}$. Hence an alternative mechanism would be adsorption of two acetylene molecules with both forming two bonds to the surface after adsorption [66]. A 
similar conclusion was derived by molecular dynamics simulations [67] using potentialenergy function developed by Brenner et al. [68]. The adsorption of different hydrocarbon species including $\mathrm{H}$ on diamond $\{111\}$ was studied using $a b$ initio molecular orbital theory $[62,69]$. In the decreasing order of adsorption to the surface, this theory predicted $\mathrm{C}_{2} \mathrm{H}>\mathrm{H} \approx \mathrm{CH}_{2}>\mathrm{CH}_{3}>\mathrm{C}_{2} \mathrm{H}_{2}$. Although $\mathrm{C}_{2} \mathrm{H}$ binds very strongly to $\{111\}$, its production in the gas phase requires more energy than $\mathrm{CH}_{3}$, hence it is not probably the most important species for diamond growth [70].

On diamond $\{100\}$ also, there is a continuing debate pertaining to growth mechanism. Harris et al. [71] proposed a growth model involving only neutral $\mathrm{CH}_{3}$ and hydrogen atoms using a nine-carbon model compound, and the predicted growth rate on the $\{100\}$ surface agreed well with the experimental values. However, the steric repulsion between the $\mathrm{H}-\mathrm{H}$ sites on the surface covered with $-\mathrm{CH}_{2}$ was not taken into account. In order to reduce this steric repulsion, the $\{100\}$ surface normally reconstructs. So, surface reconstruction could play an important role on diamond $\{100\}$ when the surface temperature of diamond exceeds $1300 \mathrm{~K}$, converting a di-hydrogenated surface to a mono-hydrogenated one [72]. Using the potential-energy functions derived by Brenner et al. [68], Garrison et al. explained the mechanism of dimer opening on the $\{100\}$ surface using $-\mathrm{CH}_{2}$ species (obtained from $\mathrm{CH}_{3}$ species of the gas phase) [73]. Another important result using MD simulations for hydrocarbon adsorption on (100) diamond was the realization that binding to radical sites is determined to a great extent by local energetics of the sites (i.e. neighbor interactions) [74]. This high site-specificity is due to the strong covalent bonding in diamond. 
Experimentally, some attempts to determine the growth species used gas phase diagnostic tools such as in situ infrared absorption spectroscopy [75], mass spectrometry [76, 77-82] and high-resolution energy electron energy loss spectroscopy (HREELS) [8386]. These studies have mainly indicated measurable concentrations of $\mathrm{CH}_{3}, \mathrm{CH}_{4}, \mathrm{C}_{2} \mathrm{H}_{2}$, and $\mathrm{C}_{2} \mathrm{H}_{4}$ in the gas phase. Among these $\mathrm{CH}_{3}$ and $\mathrm{C}_{2} \mathrm{H}_{2}$ are active gas phase species participating in the CVD. Gas phase reactions have indicated that $\mathrm{C}_{2} \mathrm{H}_{2} \rightarrow \mathrm{CH}_{3}$ conversion or the reverse reactions are also important [87]. In a microwave plasma reactor, mass spectroscopy studies indicated that the gas phase composition is independent of the inlet gas composition $\left(\mathrm{CH}_{4}\right.$ vs. $\left.\mathrm{C}_{2} \mathrm{H}_{2}\right)$, and hence the film growth depends only on the kinetics of surface reactions, which is determined by substrate temperature [80]. Similar effect was seen in a hot filament reactor, but only close to the substrate surface and at $2 \%$ or less hydrocarbon/hydrogen ratios [79].

Although a general consensus was still not achieved as to which species is responsible for diamond growth, it was somewhat agreed that $\mathrm{CH}_{3}$ and/or $\mathrm{C}_{2} \mathrm{H}_{2}$ could play such a role. Thus, the general picture of diamond CVD is that the growing surface is normally saturated with hydrogen. Atomic hydrogen $\mathrm{H}$ abstracts a surface $\mathrm{H}$ to form $\mathrm{H}_{2}$, leaving the surface with a reactive surface site. This surface site may regain its $\mathrm{H}$ atom or may now adsorb a gas phase hydrocarbon radical $\left(\mathrm{CH}_{3}\right.$ or $\left.\mathrm{C}_{2} \mathrm{H}_{2}\right)$. Thus diamond growth occurs by a step nucleation and propagation in a layer-by-layer method. On a macroscopic scale, the way the diamond layers add to the growing crystal depending upon the surface sites, determines the final faceting of individual crystals, and texturing in polycrystalline films. Obviously, the role of defect formation during growth would drastically affect the morphology of crystals and also the growth kinetics. 


\subsection{Morphology of diamond crystals}

Diamond crystal growth is direction dependent because of strong covalent bonding. Local bonding and the geometry of the surface sites play important roles in the growth kinetics and lead to different crystal morphologies. Wulff's theorem [88] states that each facet of a crystal is described by its free surface energy and the crystal shape results from minimizing this surface energy for a certain volume. Hence, only the surfaces with the lowest energy will be visible, while the ones with higher energies will shrink or disappear. However, this model is valid only for crystals grown under thermal equilibrium, and hence the resulting shape predicted is the equilibrium shape of the crystal. The conditions during the growth of diamond by CVD are far away from thermal equilibrium and hence the crystal shape is determined by growth kinetics and not necessarily by minimization of surface energy. For diamond crystals, a modified velocity-dependent Wulff construction is used to determine the equilibrium shape, where the driving force is not the equilibrium surface energy, but the growth velocity of each facet as determined by the kinetics. Thus, the fundamental factor that determines crystal morphology is the competition of growth velocities in different crystallographic directions [89].

The texture and growth morphologies of untwinned diamond crystals (crystals without stacking faults) were explained using the growth velocity ratio $\left(\alpha=\sqrt{3} \cdot v_{100} / v_{1 I I}\right)[30,91,92]$. [Note: the $\sqrt{3}$ factor comes in due to the ratio of $d-$ spacing between $\{100\}$ and $\{111\}$ in diamond]. If $\alpha$ is close to 3 , the preferential growth occurs along [100] direction leading to an octahedral crystal bounded by $\{111\}$ facets only and if $\alpha$ is close to 1 , cubic faceting occurs (crystal bounded by $\{100\}$ facets) 
(Figure 2.5). The relationship between growth parameters (methane/hydrogen ratio, temperature of the substrate) and $\alpha$ was used in the form of maps for obtaining untwinned diamond crystals in a microwave CVD system $[90,92]$ as well as a hot-filament reactor [93].

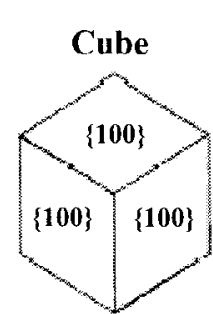

$\alpha=1$

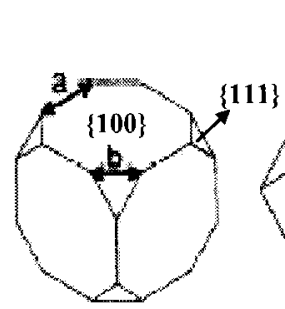

$\alpha=1.2$

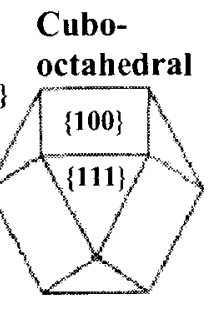

$\alpha=1.5$

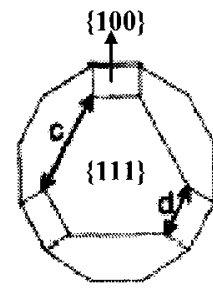

$\alpha=2$

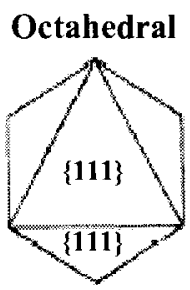

$\alpha=3$

Figure 2.5 Illustrations of different untwinned crystal shapes resulting from the change in $\alpha$-parameter from $\alpha=1$ (cubic), to $\alpha=3$ (octahedral) (Courtesy Ref [94]).

In general for diamond, triangular shaped facets are $\{111\}$ planes, while square shaped facets are $\{100\}$ planes. $\{110\}$ facets are not that well defined. Hence, as indicated in Figure 2.5, a cube is surrounded by square $\{100\}$ planes, while an octahedral is surrounded by triangular $\{111\}$ planes. The shapes intermediate between these two extremes have a combination of these two primary planes.

\subsection{Role of defects in the morphology of diamond crystals}

Studies on early stages of growth help in understanding the origin of defects during growth and their effect on morphology. Defects and morphology control are important in growing large single crystals, and single-crystal quality films with controlled texturing. 
Defects are believed to originate via a hexagonal step nucleation on $\{111\}$ surface during diamond crystal growth [95]. Carbon atoms in diamond can attach either in the staggered or eclipsed form. These stacking differ in the angle at which the subsequent atom bonds (See Figure 2.6). The eclipsed conformation leads to hexagonal stepnucleation and hence stacking faults in diamond. A perfectly staggered stacking leads to a defect-free untwined crystal, while two and three stacking errors on different $\{111\}$ planes give rise to five-fold decahedral and rounded icosahedral twinned diamond crystals $[96,97]$.

(c)
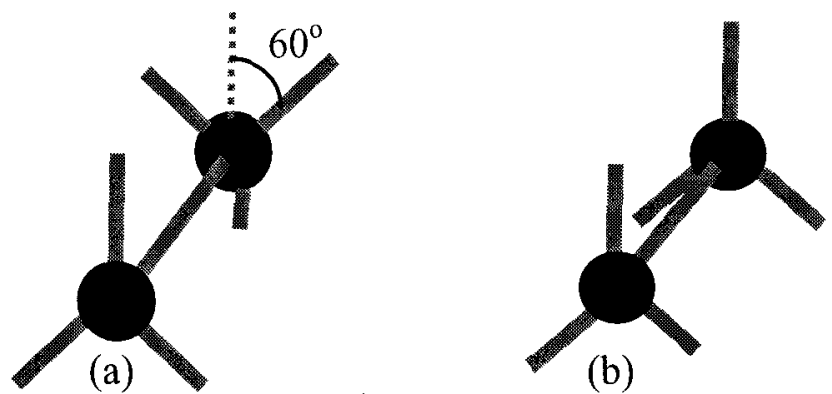

(b)

Hexagonal

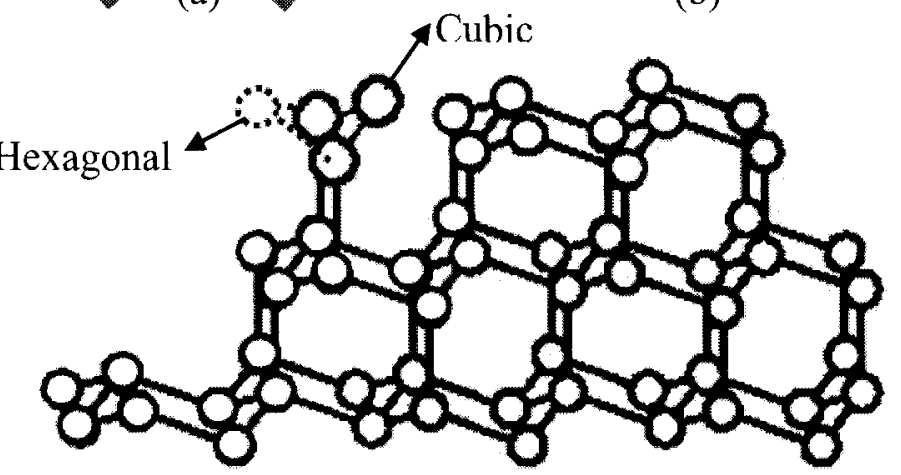

Figure 2.6 Model of two types of stacking of $\mathrm{sp}^{3}$ carbon atoms: (a) staggered-stacking and (b) eclipsed-stacking. These two types of stacking result in cubic and hexagonal-step nucleation of diamond respectively on $\{111\}$ as illustrated in (c).

Twinning and enhanced growth at twin reentrant corners were recognized as important factors affecting the morphology of CVD diamond films $[95,96]$. Scanning 
electron microscope (SEM) images of typical morphologies of twinned diamond crystals are shown in Figure 2.7. A decahedral crystal (Figure 2.7a) is characterized by one fivefold twin center, and by ten (five above and five below) $\{111\}$ facets. A do-decahedral crystal (Figure $2.7 \mathrm{~b}$ ) is similar to a decahedral crystal, but has five additional $\{100\}$ facets. A star-decahedral crystal (Figure $2.7 \mathrm{c}$ ) is also completely bounded by $\{111\}$ facets and is similar to a decahedral crystal, except that it has additional $\{111\}$ facets across the twin boundaries, giving it an overall star-shape. It also has a characteristic dimple at the five-fold axis. A star-dodecahedron is similar to a star-decahedron, except that it has five $\{100\}$ facets as well (Figure 2.7d). An icosahedral crystal (Figure 2.7e) has twenty $\{111\}$ facets and twelve five-fold centers. A dimpled icosahedron has dimples on all the five-fold centers (Figure 2.7f). Apart from these basic features, multiply twinned crystals can also have some grooves along twin planes. 

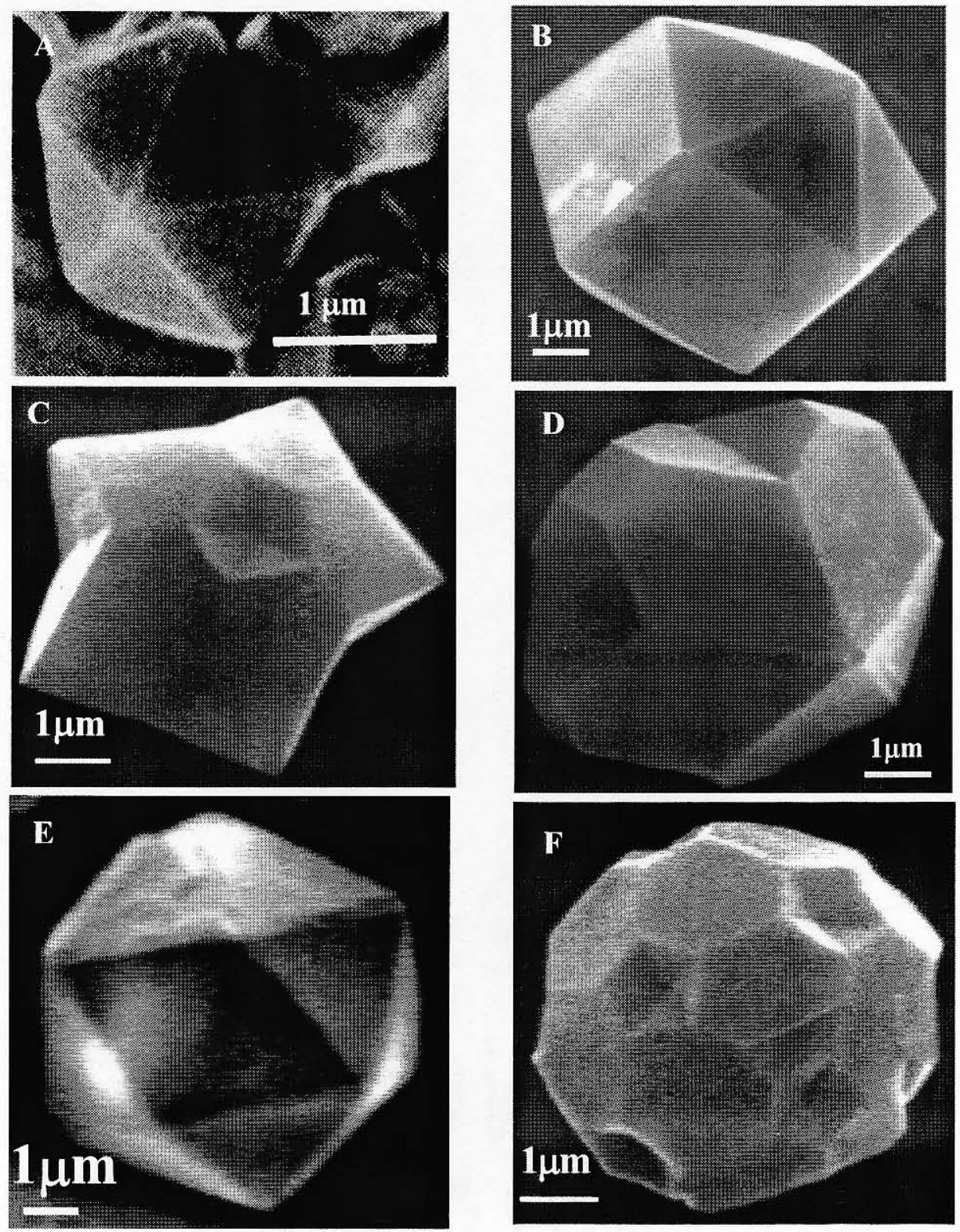

Figure 2.7 SEM images of different morphologies of multiply twinned diamond crystals:

(a) decahedron (courtesy ref. 98), (b) dodecahedron, (c) star-decahedron, (d) stardodecahedron, (e) icosahedron, and (f) dimpled icosahedron

A lot of information can be gathered about crystal growth by just observing the change in morphology of these twinned or untwinned crystals with change in growth 
conditions or with subsequent growth. For instance, perfect decahedral crystals are seen quite rarely in a microwave CVD grown crystals. Hence, the perfect decahedron shown in Figure $2.7 \mathrm{a}$ is taken from reference 98 that was grown in a hot-filament reactor. The different five-fold crystals shown in Figure $2.7 \mathrm{a}-\mathrm{d}$ are inter-convertible by changing the growth conditions, and so is the case with icosahedrons and dimpled-icosahedrons.

For twinned crystals, an attempt was made to correlate the occurrence of features such as dimples, grooves, and additional faceting based on the $\alpha$ values measured for untwinned crystals at the same growth conditions [94]. This analysis predicted that at $\alpha=1.5$, a five-fold crystal takes the form of a dodecahedron (Figure $2.7 \mathrm{~b}$ ), while a twentyfold crystal takes the form of a perfect icosahedron (Figure 2.7e). When $\alpha$ increases from 1.5 to 3 , this five-fold crystal changes to star-dodecahedron (Figure $2.7 \mathrm{~d}$ ) and finally to a star-decahedron (Figure 2.7c), developing additional $\{111\}$ facets, and dimples. An icosahedron changes to a dimpled icosahedron (Figure 2.7f) for the same changes of $\alpha$. With decrease in $\alpha$ from 1.5 to 1 , both the twinned particles develop additional grooves along the twin planes. This study did not indicate the $\alpha$-factor for a perfect decahedron.

Star-decahedral five-fold crystals are found in other crystal systems as well, such as face centered cubic system such as gold [99, 100], silver [101] and copper [102] as well as hexagonal system such as boron nitride [100]. In a star-decahedral crystal, intuitively there should be a high probability of growth at the twin boundaries, where the two additional $\{111\}$ facets meet. Hence this star-decahedron should tend to close up during growth giving rise to a decahedron. In addition, based on the definition, the $\alpha$ factor for either star- or perfect decahedrons should be the same because both are bound 
by $\{111\}$ facets. However, the conditions for the occurrences of decahedron and stardecahedron morphology seem to be different.

The kinetic pathway for the formation of a star decahedral morphology compared to decahedral morphology is not known. This allows for a unique opportunity to determine the molecular level processes that are responsible for these morphologies. To bridge the gap between the macroscopic parameters ( $\alpha$-factor) and microscopic events (growth mechanisms) on the surface, simulations at physically significant sizes of detailed chemical kinetics need to be performed.

\subsection{Simulations for kinetic modeling of large-scale crystal growth}

Kinetic modeling used for gas-surface reactions are described in terms of elementary reactions of surface species. Specific reaction rate constants for these reactions are obtained by fitting a model to experiments, or theoretically evaluating them, or by analogy to similar gaseous reactions. The difficulties lie in knowing the actual kinetic mechanism and the corresponding rate constant for various gas-surface reactions. There are several approaches attempted to solve this problem. Some of these included quantum mechanical simulations, molecular dynamics (MD), transition state theory for determining rates, and Monte Carlo simulations. MD simulations cannot be used to describe the macroscopic properties of the system that evolve over longer periods of time and larger size domains due to the limited time step size $\left(\sim 10^{-15} \mathrm{~s}\right)$ and the simulation cell size constraints $\left(\sim(100 \AA)^{3}\right)$. Thus, MD simulations carried out for diamond were restricted to small simulations e.g. determining the growth species (described in section

2.4). These difficulties on the other hand are easily overcome by MC simulations. 
However, MC simulations predict steady state properties and structures at the expense of the microscopic details of the time-dependent dynamics.

An important parameter is the rate constant of gas-surface reactions, which can be determined analogous to hydrocarbon gas-phase reactions. However, this involves an assumption that the same kinetics applies to gas-surface reactions. Molecular mechanics force fields and energy minimization programs using semi-empirical potentials derived by Brenner et al [68] have also been used to estimate the values of these gas-surface rate constants.

Time dependent Monte Carlo simulations were used to determine the steady state distribution and density of $\mathrm{C}$ radicals on the diamond $\{001\}(2 \times 1): \mathrm{H}$ surface [103] and the diamond $\{111\}$ surface $[104,105]$. The reaction rates and probabilities of each individual event was either explicitly calculated via molecular dynamics or taken from experiments. However, due to the complexity of the simulations, the cell sizes used in these simulations were small, or the growth rate achievable was very small.

Kinetic Monte Carlo (KMC) simulations to explain diamond film growth in [100], [111] and [110] orientations were carried out by Battaile et al [106-109]. For the first time, a large-scale film growth simulation was performed for $\{111\},\{110\}$ and $\{100\}$ surface with $\mathrm{CH}_{3}$ and $\mathrm{C}_{2} \mathrm{H}_{2}$ gas phase species using rate constants from literature for gasphase reactions and ones calculated by transition state theories. These simulations tabulated a set of reactions on specific surface sites. Using a random number generator and the linear scale of probability of reactions, a reaction on a specific site is chosen. The determined growth rates from these simulations gave a reasonable match with 
experimentally determined values. However, no significant information on the overall morphology of the films grown by simulations could be gathered from these simulations.

The chemical nature of the site as well as its location on the surface, the number and the nature of its neighboring sites must also be considered when modeling surface reactions. With the KMC technique, one can postulate a set of rate constants with any number of reactions to account for all the species interactions with the surface. The validity of these postulated rate constants can be checked by comparing the final simulated morphology and resulting kinetics to the experimentally observed morphology and growth rates. A KMC technique using simple single adatom addition was used to explain the morphological evolution of untwinned crystals (octahedron, cubo-octahedron) and multiply twinned (decahedron, do-decahedron and icosahedron) diamond crystals [97]. However, this methodology with the chosen chemistry was not adequate to differentiate between a star-decahedral morphology and decahedral morphology.

A more rigorous set of reactions are probably needed to simulate the kinetic faceting of multiply twinned particles such as presence of additional $\{111\}$ facets giving rise to a star-shape, reasons for the existence of dimples and grooves, etc. Specifically, the kinetic preference of a star-decahedral morphology to a perfect decahedral morphology constitutes the motivation for the studies of early stages of growth.

\subsection{Morphological manifestations of $\mathrm{sp}^{2}$ carbon crystals}

Graphite is another important allotrope of carbon widely used for a variety of applications e.g. lubrications, conductive surfaces, heat exchangers for good thermal conductivity, electrodes, fuel cells as catalysts, for seals and gaskets, just to name a few. 
Graphite is characterized by planar $\mathrm{sp}^{2}$ bonding, and hence has a layered structure. The nearest neighbor distance between carbon atoms in graphite is $0.144 \mathrm{~nm}$, while the interplanar distance is $0.33 \mathrm{~nm}$. Graphite exits in a wide range of one, two and threedimensional morphologies. Although graphitic whiskers, few hundreds of nanometers in dimensions, were discussed as early as 1960 [110], their atomic structure could not be understood clearly due to the lack of high resolution electron microscopy facilities. The discovery of fullerenes (C60 cage compounds) pioneered the research efforts on novel carbon nanostructures [1]. A detailed structural investigation of one-dimensional carbon nanostructure (carbon nanotubes) was presented by lijima in 1991 [111]. This work pioneered efforts in understanding the rolled graphene structure and a wide variety of carbon nanostructures were proposed since. Figure 2.8 shows the three main forms of graphite, bulk graphite, bucky ball (C60) and capped-single walled nanotube (SWNT). Several concentric SWNTs form multi-walled carbon nanotubes (MWNT). The characteristic feature of bucky balls and carbon nanotubes is that the $\pi$-conjugated carbon hexagon rings with the participation of some pentagon rings form rolled-up closed surfaces. Thus, they have negligible edge sites present, unlike bulk graphite that has basal plane and edge plane sites (except when the caps of the CNTs are opened). An excellent review for the discovery and synthesis of $\mathrm{sp}^{2}$ carbon nanophases can be found in references [35-37]. 


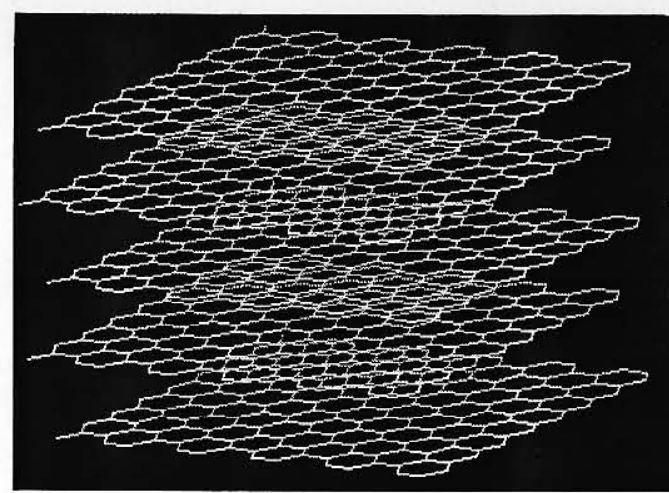

(a)

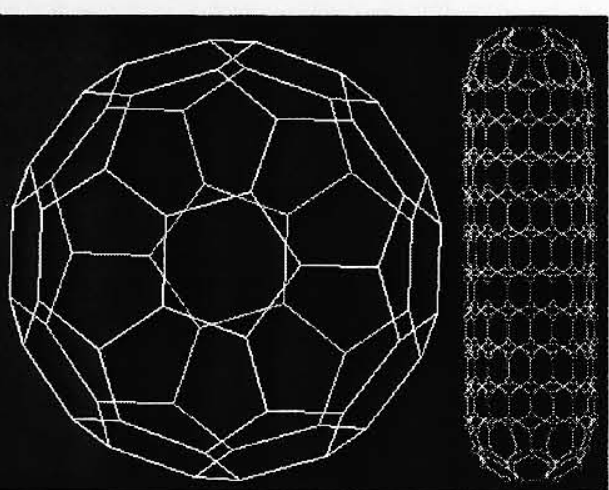

(b)

(c)

Figure 2.8 Ball and stick model of (a) bulk-graphite with basal planes and edge planes, (b) C60 molecule, and (c) single-walled carbon nanotube.

Among other nanoparticulate (3-D) forms of graphite are carbon aerogels, metallofullerenes and onion-like carbon. Carbon aerogels are highly conductive (electrically) porous materials with high surface area formed by interconnected nanometer sized particles $(3-30 \mathrm{~nm})$ with small interstitial pores $(<50 \mathrm{~nm})$ [112]. These aerogels have soot-like character. Metallofullerenes or nanocapsules are metal or metal carbide encapsulated carbon shells $[113,114]$. Onion-like carbon shells (with hollow core) were synthesized by graphitizing nanocrystalline diamond particles by electron beams in a high resolution TEM. These structures had concentric round shells of graphite [115]. While, polyhedron closed-carbon shells were reported by a similar synthesis technique of heating nanosized diamond crystals in inert argon atmosphere [29].

The synthesis of nanocrystalline graphite was achieved by several means such as high energy laser-pulse [116], mechanical milling [117], ball milling for long durations 
[118], and thermal chemical vapor deposition (CVD) from methane/hydrogen precursors [119]. However, little is known about the three-dimensional structure of these graphite nanocrystals or the electronic structures due to their high edge-site densities.

The presence of edges in polyhedron closed-carbon shells of graphite was recognized as being important for enhancement of density of states due to the presence of non-bonding $\pi$-electrons [29]. If this were true, then nanosized bulk-graphite crystals could be imagined to have much higher edge-site density than polyhedral graphite shells.

Similarly a myriad of structural manifestations of 1-D carbon nanostructures were reported. Followed by Bacon's graphite structures [110], conical graphite whiskers were synthesized by heating SiC crystals [120]. Both these conical graphite structures had similar morphology. The structural model proposed for this morphology was the wrapping up of graphite sheets around the whisker axis (Figure 2.9a), producing a scroll structure. It may intuitively seem that the inner channels of these structures may be hollow, but no evidence was presented to support this argument. In addition, these crystals needed to be extracted from a matrix of carbon material (or $\mathrm{SiC}$ in the later case). Single-walled graphite fullerene cones were synthesized using pyrolysis of hydrocarbons (Figure $2.9 \mathrm{~b})[121,122]$. These cones are formed due to the specific combination of pentagonal and hexagonal rings. Based on this model, closed cones with angles $19.2^{\circ}$, $38.9^{\circ}, 60^{\circ}, 86.6^{\circ}$ and $123.6^{\circ}$ were known to occur. Carbon nanohorns [123] were synthesized by $\mathrm{CO}_{2}$ laser ablation of carbon without the presence of any metal catalyst. Horn-shaped sheaths of single-walled graphite were found bundled-up in spherical clusters (Figure 2.9c). There were called nanohorns. Graphite polyhedral crystals [124] and conical crystals [125] were discovered by fracturing the surface of glassy carbon. 
These have graphite crystals had a well-ordered structure made up of parallel graphite sheets, but with closed ends (see Figure 2.9d). Graphite whiskers, with a scroll structure, were synthesized by high temperature treatment of milled graphite (see Figure 2.9e). These whiskers have carbon layers almost perpendicular to the whisker axis and grow by disinclination growth mechanism [126]. Another unique morphology is with graphite sheets parallel to the cone axis, called carbon nanotips [127] shown in Figure 2.9f, synthesized by CVD on biased Ti-Pt coated silicon substrates. These structures were not reported to have any hollow channels.

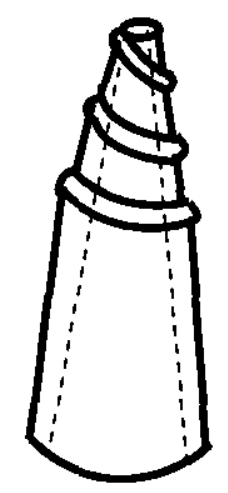

(a)

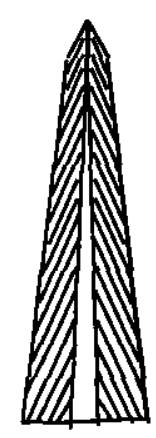

(d)

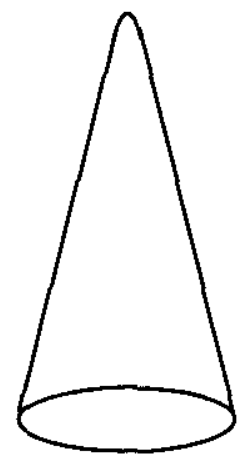

(b)

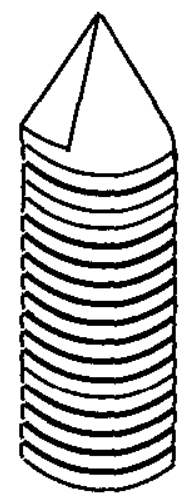

(e)

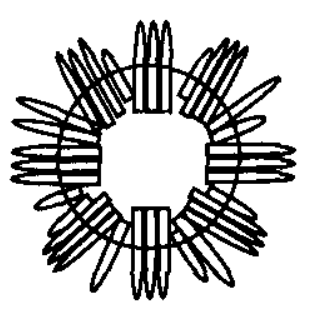

(c)

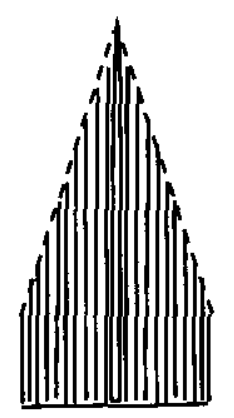

(f)

Figure 2.9 Illustrations of different morphologies of one-dimensional conical carbon nanostructures. 
The above-presented conical nanostructures are in essence different morphological manifestations of carbon nanotube, also formed by rolled up graphite sheets. Conical morphologies of graphite nanostructures are interesting because they have a combination of micro and nanoscale properties. They are more rigid than carbon nanotubes, because of their thick walls and large base diameters (typically in the order of microns), yet have a nanoscale tip. Conical hollow morphologies that are open-ended, present an added advantage with possible fluid flow applications. However, all the conical morphologies reported above have only produced closed-ended, short and scattered nanostructures. This could be because there has not been any systematic study on the growth mechanisms that give rise to these conical structures. Little is known about the morphological control of these nanostructures, or growing them in the form of ordered arrays. There is a need for systematic growth studies to clearly define the gassurface mechanisms that are responsible for these useful morphologies of onedimensional structures, particularly conical carbon nanostructures.

The attractive feature of nanomaterials is not only their applications due to nanoscale, but also the change in properties of materials at the nanoscale. For instance, bulk graphite is very soft, while carbon nanotubes have an elastic modulus much higher than steel $(\sim 350$ times stiffer than steel). The characteristic surface sites of these conical structures are different from the rolled up basal planes of graphite found in nanotubes. The advantage of these multi-walled conical structures over nanotubes is their rigidity and surface activity on account of the large edge-site density (except for Figure 2.9b, c). 
It will be desirable to be able to synthesize controlled morphologies of carbon nanostructures, which have the benefits of carbon nanotubes, but in addition also have rigidity and surface activity, for some useful applications like sensing and fluid flow.

\subsection{Summary}

The technological development in the growth of single crystal and single-crystal quality diamond has been significant; yet, the understanding of nucleation and growth is limited. A number of studies on $\mathrm{sp}^{2}$ and $\mathrm{sp}^{3}$ carbon nanocrystals have suggested possible phases, which are not known to exist in the macroscopic scale (such as n-diamond and icarbon). There is no information on the exact structure of these phases. Studying the structure of nanocrystals of diamond (few $\mathrm{nm}$ in size) could bring about a better understanding on diamond nucleation and the possible discovery of new phases of material.

Oriented films of CVD diamond have been successfully synthesized, but better understanding is needed of the chemical kinetics that controls the morphology of individual diamond crystals during growth from the vapor phase. The difference in growth rules that govern the morphology of different forms of five-fold twinned crystals such as star-shaped decahedral and decahedral crystals is not yet known. Understanding the link between microscopic events and macroscopic features of crystal morphology is necessary to understand how to control and improve the quality of CVD grown diamond films and single crystals.

Nanocrystalline forms of $\mathrm{sp}^{2}$ carbon have attracted much interest - specifically for carbon nanotubes. There are several morphological manifestations of carbon nanotubes, 
in the form of conical carbon nanostructures, being synthesized. Yet, there have not been any reports of open-ended conical carbon nanostructures. If the morphology of these one-dimensional graphitic structures could be controllably synthesized in the form of arrays, some useful applications in nanofluidics and sensing could be realized based on their morphologies. 


\section{CHAPTER 3}

\section{EXPERIMENTAL AND ANALYTICAL PROCEDURES}

\subsection{Introduction}

In this chapter, procedures used for studying nucleation and early stages of growth of carbon crystals are described: a simple procedure of capturing a large density of diamond and graphitic nanocrystals and interrupted growth experiments to study early stages of diamond crystal growth from vapor phase. The structural characterization of nanocrystalline materials is not possible with just one technique, but needs a combination of different techniques. Thus, this work utilizes an array of characterization tools ranging from electron microscopy to electrochemical characterization for single nanocrystals to bulk materials. Scanning electron microscope (SEM) and atomic force microscopy (AFM) were primarily used for observing the morphology of the micro/nano crystals. The nanocrystals were then analyzed for their composition using Electron dispersive spectroscopy (EDS), and crystal structure using Transmission electron microscopy (TEM) and X-ray diffraction (XRD). Carbon phase identification was performed by Raman spectroscopy. Surface structures of the nanocrystals were analyzed using X-ray photoelectron spectroscopy (XPS). Electroanalytical techniques such as cyclic voltammetry (CV) and electrochemical impedance spectroscopy (EIS) were used to understand the electrochemical behavior of carbon nanocrystalline films. Each of these characterizations tools will also be briefly discussed. The Kinetic Monte Carlo 
technique used to model the growth of diamond crystals is discussed in detail later in Chapter 5 and will not be discussed here.

\subsection{Nucleation experiments}

Nucleation experiments were carried out in a model 5010 ASTeX microwave plasma chemical vapor deposition (MW CVD) reactor. A sketch of the CVD reactor is shown in Figure 3.1a. This reactor is equipped with a $1.5 \mathrm{~kW}$ microwave generator. In general, a plasma (ionized gas consisting of positively charged ions and negatively charged electrons) is generated by subjecting molecules to severe conditions such as intense heat. Microwave (electromagnetic radiation) is one such a source of ionizing gas molecules. Due to the interactions of a high-frequency electric field $(2.45 \mathrm{GHz})$ in the form of microwaves with a low-pressure gas, a glow discharge is produced. Electronic transitions in excited state molecules produce radiation, which causes the plasma region to glow (Figure 3.1b). The electrons are accelerated to very high energy levels, corresponding to $5000 \mathrm{~K}$, while heavier ions do not respond as quickly and hence remain at significantly lower temperatures $(<2000 \mathrm{~K})$. Due to this non-uniformity in electron and ion temperatures, the plasma is non-isothermal. Collision of these electrons with gas molecules in the reactor causes further excitation of the molecules and production of neutral and ionic species. These ionic/neutral species are responsible for the growth of the solid material on the substrate from the gas phase.

The MW CVD reactor used in this dissertation does not have independent substrate heating capability. However, the temperature can be manipulated by a combination of pressure in the reactor camber and the microwave power. 


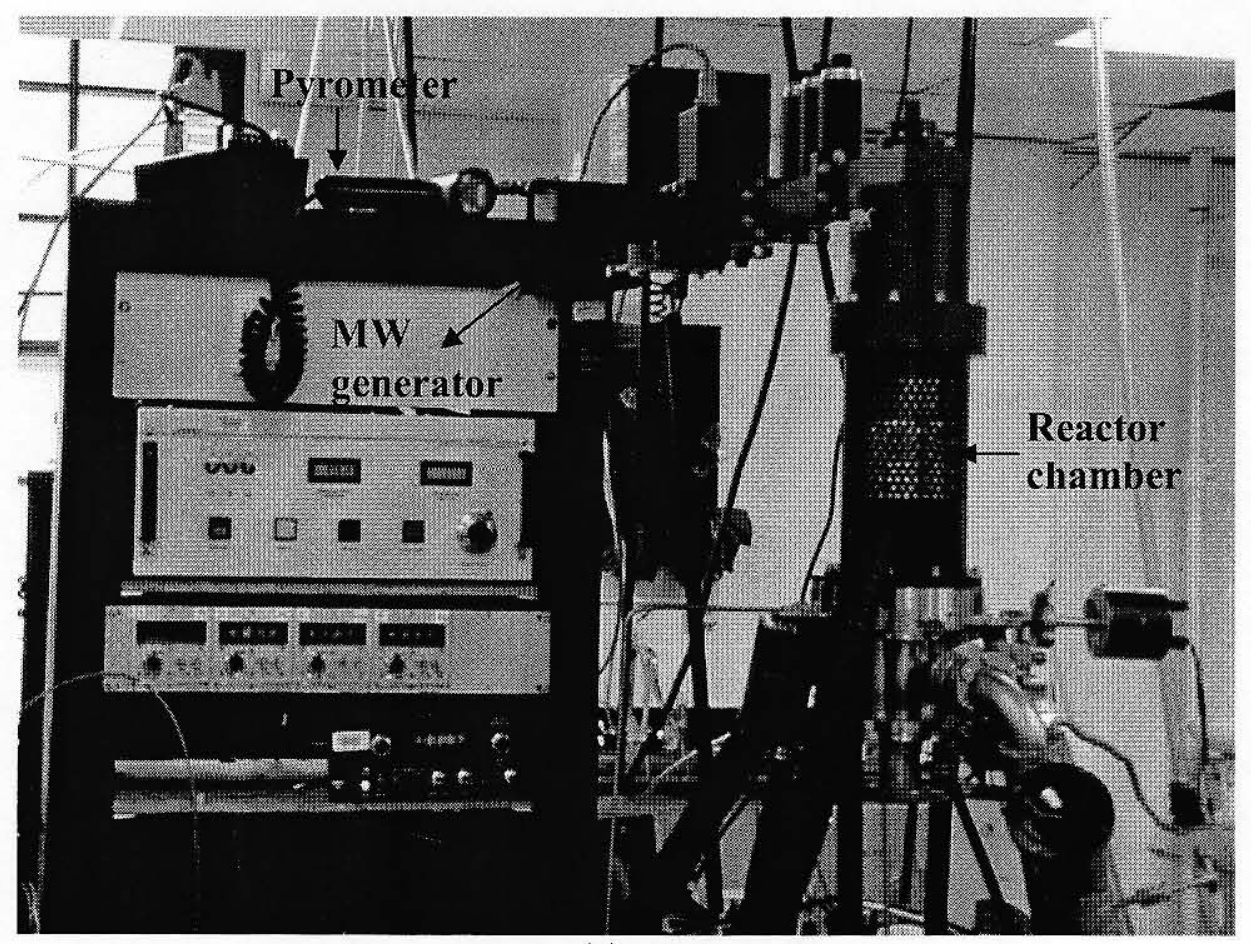

(a)

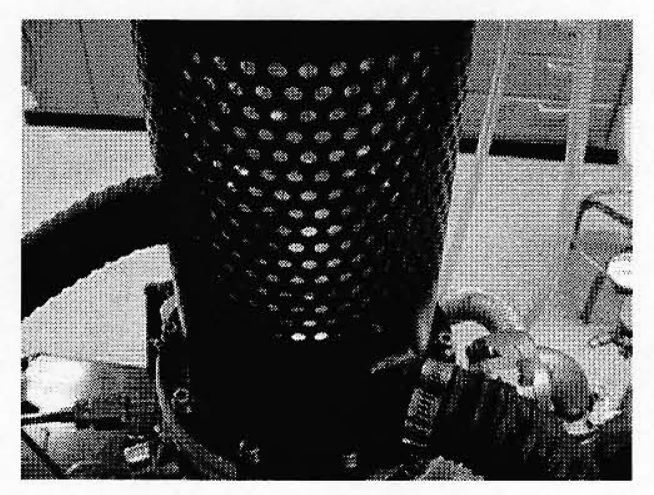

(b)

Figure 3.1 Digital images of (a) 5010 ASTex Microwave plasma CVD reactor and (b) side-view the quartz chamber with hydrogen plasma (purple glow) above the substrate.

A simple and effective means of capturing a high nucleation density of carbon nanocrystals was designed by immersing metal substrates directly into the dense zone of the plasma (Figure 3.2a). Platinum wires $(0.0254 \mathrm{~cm}$ dia, $3 \mathrm{~cm}$ length), were used as the 
substrates. They were scratched mechanically with diamond powder $(0-2 \mu \mathrm{m})$ and then ultrasonicated in acetone. The platinum wires were placed into a multiple-wire graphite holder, which had several slots to hold platinum wires vertically (Fig. 3.2 (b), (c)). This holder was then placed on the substrate stage and inserted into the reactor. The reactor chamber was evacuated to a few millitorr base pressures using a roughing pump. Sufficient care was taken to minimize any oxygen leak (through air) into the reactor. 2 $\mathrm{sccm}$ of methane and $200 \mathrm{sccm}$ of hydrogen were introduced into the reactor, maintaining a pressure of 50 Torr. The plasma was obtained at $1100 \mathrm{~W}$. These plasma conditions are typical for diamond growth conditions.

Since the wire-substrates were directly immersed into the microwave plasma, the plasma position seemed to have changed slightly. Such a change in the microwave plasma position relative to the substrate has been observed before [82]. As soon as the plasma was obtained, the platinum wires tended to melt down near the tip into a ball shape. The melting of the platinum wire was sensitive to its length exposed in the plasma. Typically, it was observed that if the Pt wire length was lesser than $1 \mathrm{~cm}$, the wire tip did not melt into a ball. The tip of the platinum wires glowed in the plasma. The temperature at the tip of the wire was measured using a single wavelength optical pyrometer (Raytek Model RAYMA2SCCF), and was close to the melting point of platinum $(\sim 2000 \mathrm{~K})$. Hence, at the end of long-term (24 hours) experiments, a large density of carbon nanocrystals was formed at the tip of the wire.

Since the platinum wires were immersed into the plasma, there was a significant variation in temperature and plasma density along the platinum wire. Hence, this synthesis strategy also allowed the study of deposition of different phases of carbon 
(carbon nanocrystals at the tip, and 1-D carbon nanostructures away from the tip) along the length of the same platinum wire. To study the growth mechanisms of these 1-D structures, short term experiments were also carried out ( $\sim 1$ hour experiments).

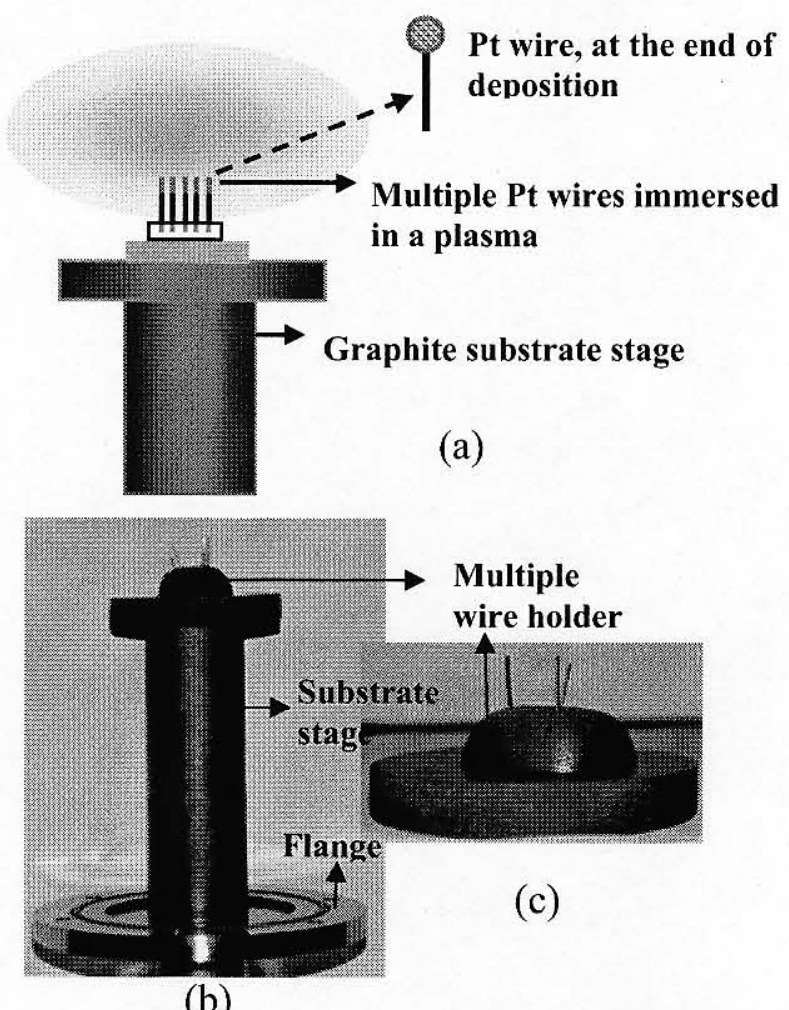

Figure 3.2 (a) A schematic of multiple platinum wire immersion into a MW plasma. Digital image of (b) graphite substrate stage supported by a steel flange and (c) platinum wires sticking out of the multiple wire-holder.

Using the same gas phase composition and plasma conditions of 50 torr pressure, $1100 \mathrm{~W}$ power and $1 \% \mathrm{CH}_{4} / \mathrm{H}_{2}$, but with platinum wires placed horizontally on the graphite substrate stage (without the multiple-wire holder), a uniform diamond film was obtained after several hours of growth. Typically several pieces of solid boron (few $\mathrm{mm}$ in size) were placed in the vicinity of the silicon substrate on the graphite substrate stage. 
The growth rate achievable in this reactor for diamond at the plasma conditions described above is $1 \mu \mathrm{m} / \mathrm{hr}$.

The synthesized carbon nanocrystals and 1-D carbon nanostructures were analyzed using Scanning electron microscope (SEM), Energy dispersive $x$-ray spectroscopy (EDX), X-ray diffraction (XRD), Raman Spectroscopy, Transmission electron microscopy (TEM) and X-ray photo-electron spectroscopy (XPS).

\subsection{Interrupted growth experiments}

Interrupted growth experiments were carried out for studying the morphology change during early stages of diamond growth. Diamond crystals were grown on an unseeded $\mathrm{Si}\{100\}$ or $\{111\}$ substrates $(1 \mathrm{~cm} \times 1 \mathrm{~cm})$ using $1 \% \mathrm{CH}_{4} / \mathrm{H}_{2}$ plasma at 50 torr and $1100 \mathrm{~W}$ in the MW CVD reactor. A very low density of diamond crystals was obtained on the $\mathrm{Si}$ substrate by preventing the use of any nucleation enhancement procedures like scratching or seeding the substrate. The nucleation density was intentionally kept low to observe the morphological evolution of each individual crystal over 4-5 re-growth experiments (and hence prevent film formation). The temperature measured using the optical pyrometer was $700^{\circ} \mathrm{C}$.

The individual micron-sized diamond crystals were mapped on the substrate, using a SEM, by making a note of its position relative to the overall substrate and by taking several low magnification images of its neighborhood. This relatively simple technique was successful most of the times in tracking the same crystals on the substrate after consecutive experiments. The crystals were then re-grown in the reactor for very short durations (1-1.5 hours) at same or different conditions, to observe their change in 
morphology with time or with growth conditions. Extreme was taken not to ruin the surface of the sample, as any contamination in the form of dirt could affect the re-growth experiments. Typically, before every re-growth, the sample surface was cleaned for about 30 mins using hydrogen plasma. Since the current reactor set-up lacks independent temperature control, only power and pressure were used to control substrate temperatures. Often, during re-growth experiments, secondary nucleation occurred and the individual crystals converged to begin polycrystalline film formation. Thus, change of growth conditions like temperature, pressure and methane concentration in the gas phase were cautiously chosen such that after re-growth, the crystals could be tracked and re-imaged.

In addition to imaging crystals using SEM, AFM was also used a few times to measure the angles between the facets of the crystals.

\subsection{Nanomaterials characterization}

The characterization techniques, their purposes (in this work) and the length scales that they are useful in analyzing is presented in Table 3.1 
Table 3.1 Nanomaterials characterization techniques.

\begin{tabular}{|c|c|c|c|}
\hline Technique & Purpose & Sampling area & Resolution \\
\hline SEM & Morphology of crystals & $1 \mathrm{~mm} \times 1 \mathrm{~mm}$ & $20 \mathrm{~nm}$ \\
\hline EDS & Elemental analysis & $\begin{array}{l}3 \times 3 \mu \mathrm{m} \text { and } \\
1 \mu \mathrm{m} \text { depth }\end{array}$ & $\begin{array}{l}\text { Atomic wt. > } \\
\text { Boron }\end{array}$ \\
\hline $\begin{array}{l}\text { TEM (Imaging, } \\
\text { Selected area } \\
\text { diffraction) }\end{array}$ & $\begin{array}{l}\text { Phase identification, structural } \\
\text { characterization using diffraction } \\
\text { pattern from a selected region }\end{array}$ & $\begin{array}{l}\text { Grid size } 5 \\
\mathrm{~mm} \text { dia }\end{array}$ & $\begin{array}{l}1 \mathrm{~nm} \text { for } \\
\text { imaging, few } \\
\mathrm{nm} \text { for SAD }\end{array}$ \\
\hline $\begin{array}{l}\text { TEM } \\
\text { (Nanodiffraction) }\end{array}$ & $\begin{array}{l}\text { Structural characterization using } \\
\text { diffraction from a single crystal }\end{array}$ & $<1 \mathrm{~nm}$ & $<1 \mathrm{~nm}$ \\
\hline XRD & $\begin{array}{l}\text { Power diffraction pattern of a bulk } \\
\text { sample }\end{array}$ & Tens of $\mathrm{mm}$ & $\begin{array}{l}\text { Full sample } \\
\text { area used }\end{array}$ \\
\hline AFM & Surface topography & $5 \mu \mathrm{m} \times 5 \mu \mathrm{m}$ & $\begin{array}{l}10 \mathrm{~nm} \text { lateral, } 1 \\
\mathrm{~nm} \text { step height }\end{array}$ \\
\hline $\begin{array}{l}\text { Raman } \\
\text { spectroscopy }\end{array}$ & $\begin{array}{l}\text { Phase identification }\left(\mathrm{sp}^{2} \text { or } \mathrm{sp}^{3} \text { for }\right. \\
\text { carbon) }\end{array}$ & $\begin{array}{l}2 \mu \mathrm{m} \times 2 \mu \mathrm{m}, \\
1 \mu \mathrm{m} \text { depth }\end{array}$ & $6 \mathrm{~cm}-1$ \\
\hline XPS & Surface chemical analysis & $\begin{array}{l}1 \mathrm{~cm} \text { square } \\
\text { area and } 10 \\
\mathrm{~nm} \text { depth }\end{array}$ & $0.04 \mathrm{eV}$ \\
\hline $\begin{array}{l}\text { Electroanalytical } \\
\text { technique (CV, } \\
\text { EIS) }\end{array}$ & $\begin{array}{l}\text { Differentiate between different } \\
\text { type of carbon surface sites based } \\
\text { on } \Delta E_{p}\end{array}$ & $\begin{array}{l}\text { Complete } \\
\text { immersed } \\
\text { sample area }\end{array}$ & $\begin{array}{l}\text { Top few layers } \\
\text { of electrode }\end{array}$ \\
\hline
\end{tabular}




\subsubsection{Electron microscopy}

The interaction of electrons with a specimen can broadly be described as two classes (1) elastic events, in which the electron beam passing through the sample does not experience any loss in energy compared to the incident beam (2) Inelastic events, which result in a transfer of energy to the solid specimen, leading to the generation of secondary electrons, Auger electrons, characteristic and continuum x-rays, long-wavelength electron-hole pairs, lattice vibrations (phonons), and electron oscillations (plasmons) [128]. Figure 3.3 clearly describes some of these interactions.

Typically, in electron microscopes a high negative voltage (typically $200 \mathrm{keV}$ in TEM and 2-25 keV in SEM) is applied between the electron source and a nearby earthed anode disc, which accelerates the electrons towards the specimen. Electron microscopes are generally required to operate under high vacuum to prevent the interaction of the gas molecules with the electron beam.

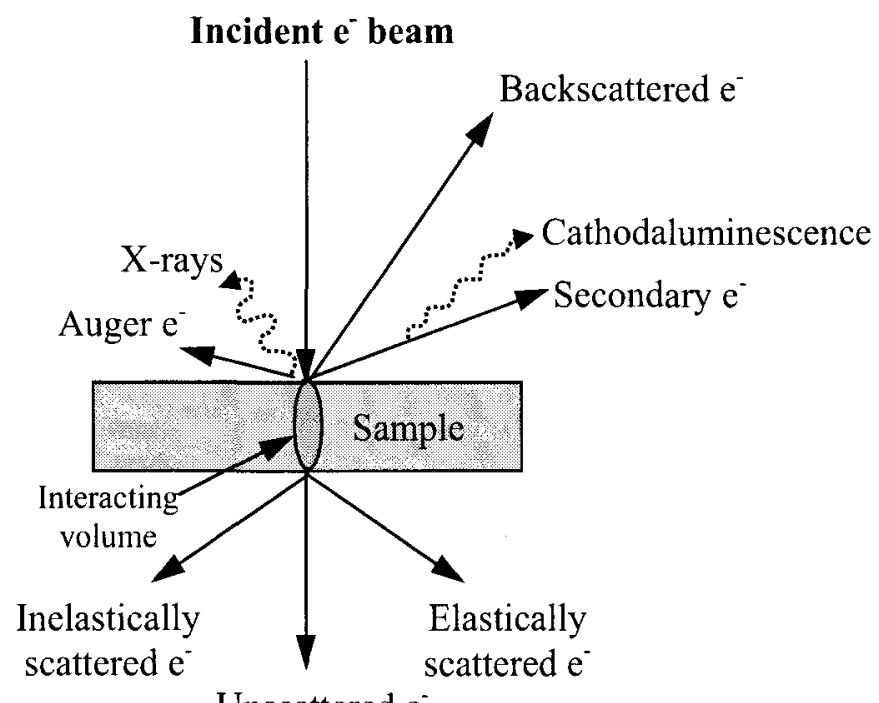

Figure 3.3 A schematic showing various processes that result from electron beam interactions with a specimen 


\subsubsection{Scanning electron microscope (SEM) and Electron dispersive X-ray spectroscopy (EDS)}

SEM uses secondary electrons, and back scattered electrons to provide contrast for images of the sample. The number of secondary or back-scattered electrons varies with the difference in surface topography as the electron beam is swept across the specimen. A JEOL model 5310 with a thermionic (Tungsten filament) electron source was used for studying the morphology of carbon micro/nanocrystals. For the JEOL model used, the (practical) limit of spatial resolution was about 10-20 nm.

The JEOL 5310 SEM is also equipped with an EDS (energy dispersive $\mathrm{x}$-ray spectroscopy) detector. When the electron beam interacts with an atom and causes the ejection of an inner-shell electron, the outer-shell electrons rearrange. When the outer shell electrons drop down to an inner shell, the atom is excited and has excess energy. One way to lose this energy is by X-ray emission. This X-ray is characteristic of each electron transition of a specific element, and hence can be used for elemental mapping of the specimen. The emitted X-rays are collected by the EDS detector.

However, the X-rays are collected from $3 \mu \mathrm{m} \times 3 \mu \mathrm{m}$ area and $1 \mu \mathrm{m}$ depth. Also, the detector is able to map elements only with atomic weight greater than Boron in the periodic table. Due to these limitations, one may use EDS as a preliminary elemental analysis tool, but must confirm the conclusions using higher resolution analysis available in a transmission electron microscope.

Further details on scanning electron microscopy and x-ray microanalysis can be found in reference [128]. 


\subsubsection{Transmission electron microscope}

TEM is capable of high-resolution imaging (approaching $1 \AA$, which is at least two orders of magnitude higher than an SEM) and structural analysis of a specimen. However, this is accompanied by the demand of having high energy electron source and a sufficiently thin (electron transparent) sample as it involves the electrons that transmit through the sample. TEM was mainly used in this dissertation for carbon phase identification.

TEM is generally operated in two modes, the imaging (for morphology) and diffraction (for structural analysis). In the fixed-beam TEM, electrons scattered by the irradiated sample are collected over a narrow solid angle and focused by the objective lens onto the image plane. The elastically scattered electrons, i.e., those which have changed their direction but not lost any energy on interaction with the specimen, interfere with the unscattered electrons to produce a phase contrast image. The inelastically scattered electrons, i.e., those that have changed both direction and energy after interaction with the specimen, generate a diffuse background image. In some TEMs, a special feature called energy filtered (EF) imaging is available, in which one selects electrons which have lost a certain amount of energy in inelastic scattering processes, and creates an image with those electrons only. Since the energy loss spectrum of a material contains a signature of all the chemical species present, an elemental map can be obtained.

The carbon 1-D structures synthesized were scraped on a TEM grid and analyzed using a JEOL 2010F TEM, which was capable of EF-TEM. 
Selected area diffraction (SAD), as the name suggests, is used to obtain electron diffraction pattern (spot pattern) from a relatively large selected region of the sample (1$10 \mu \mathrm{m})$. Each spot in the diffraction pattern is representative of a crystallographic plane. Based on the symmetry of a crystal, and based on diffraction physics, only certain reflections (spot on the diffraction pattern) are allowed, while the rest are forbidden. Thus, after obtaining a SAD pattern, it is indexed to determine the exact planes each spot represents. The term zone axis refers to the beam direction along which the spot pattern is taken. SAD is a very useful technique to obtain phase information. For more information on this technique, the reader may consult reference [129].

\subsection{Nanodiffraction using an STEM}

Another modification of electron diffraction is the convergent beam electron diffraction (CBED) technique. In CBED, the electron beam is converged to a small area of less than $100 \mathrm{~nm}$ on the specimen. Hence, CBED is capable of giving crystallographic information (diffraction and precise lattice parameters) of a highly localized region of the specimen.

Nanodiffraction is a further modification of CBED. A field emission gun (an advanced electron beam source) is necessary for this technique to obtain a high intensity $1 \mathrm{~nm}$ diameter electron beam. The electron beam is converged to a diameter of less than $1 \mathrm{~nm}$ (typically $0.2-0.7 \mathrm{~nm}$ ), and hence atomic-scale information can be obtained through this specialized technique. This technique was pioneered by Cowley et al in 1981 [130]. In nanodiffraction, due to the small beam size, getting an image of the specimen would be impossible without spreading the convergent beam. 
In order to eliminate this deficiency, a scanning transmission electron microscope (STEM) is used. In STEM, an image is generated by scanning a focused electron beam across the sample. All the electrons scattered from the sample are collected by a variety of detectors placed underneath the sample. Various types of signals discriminated in scattering angle and/or energy loss yield different structural and chemical information and may be captured simultaneously in different channels in an STEM. Thus, by using a STEM in nanodiffraction mode, one can obtain atomic-scale crystallographic information from exactly the region of interest. More information on each of these TEM techniques may be obtained from reference [129].

Electron nanodiffraction patterns were recorded with electron beams of $100 \mathrm{keV}$ energy and diameter at the specimen level of $0.7 \mathrm{~nm}$, using a STEM instrument from VG Microscope, Ltd. (UK). For the carbon nanocrystalline films synthesized in this work, without destroying the dense deposit, just the surface was analyzed using STEM. Careful analysis was needed for performing diffraction from just the top few electron transparent monolayers. Analysis of the bulk of this carbon deposit was done by crushing and placing it on TEM grids. The usual amorphous carbon supporting films were not employed because such films can introduce confusion with the amorphous and other components of the specimen. Low-magnification, point-projection ("shadow") images were used to locate suitable specimen areas. By scanning the beam over the specimen, bright-field images or dark-field images obtained with diffraction spots could be recorded. The diffraction patterns were recorded by direct photography from a viewing screen or by recording with a VCR for photography later. The latter means was well suited for obtaining series of patterns from adjacent regions during a slow scan of the 
incident beam, or for recording transient patterns from regions subject to radiation damage.

\subsection{Simulations of electron diffraction using Mac Tempas ${ }^{\circledR}$ and Crystal Kit ${ }^{\circledR}$}

It is straightforward to determine the expected spot pattern (diffraction pattern) of a material with a perfect crystal structure. Once the crystal symmetry is known, and the basis atoms of the cell are known $(\mathrm{u}, \mathrm{v}, \mathrm{w}$ positions of the smallest repeating unit of the crystal) one can calculate the structure factor $(\mathrm{F})$ from equation 3.1 .

$$
F_{h k l}=\sum_{1}^{N} f_{n} \exp \left(2 \pi i\left(h u_{n}+k v_{n}+l w_{n}\right)\right),
$$

where the summation extends over all the $\mathrm{N}$ atoms of the unit cell and $\mathrm{f}$ is the atomic scattering factor. $\mathrm{F}$ is thus a complex number. Generally, the intensity of a spot on the diffraction pattern depends on the square of the value of $F$. Hence, if $F$ is zero, the spot is said to be "kinematically forbidden". Details of this simple math for important crystal systems may be found in reference [131].

However, it is difficult to calculate the diffraction pattern of a crystal structure with large number of defects, or with mixed phases. Such calculations are very extensive. Roar Kilaas et al. at the National Center of Electron Microscopy (NCEM), Berkeley, CA developed a software called "Mac Tempas" that simulates the transmitted electron microscopy image and diffraction pattern of finite clusters that are not easily accomplished by simple calculations.

The basic working principle of the software is described here. The details can be found in the manual for Mac Tempas [132]. The multi-slice method is the most common technique for dealing with a sample with large number of defects, stacking faults, or 
interfacial structures. An electron beam in the form of a wave traverses through the electrostatic potential in a crystal. The interaction between the electron beam of a given energy and the crystal potential is given by Schrödinger equation. The solution to this equation gives the image (high resolution image) as well as the diffraction pattern. In the multi-slice method, an approximate solution to the electron wavefunction is found at the depth of $z+d z$ in a crystal from the wave function at $z$. Thus, starting with the wave function at $\mathrm{z}=0$, one can iteratively calculate the wave function at a thickness $\mathrm{n}$ dz, by applying the multislice solution slice by slice, taking the output of one calculation as the input for the next. The thickness of the slice chosen is important and one can obtain higher order reflections by reducing the thickness of the slice.

Diffraction simulations using Mac Tempas were used to predict the diffraction patterns, and intensity distribution for diffraction spots using variations in crystal structures. Clusters with large number of defects, stacking faults, mixed phases, etc were simulated using a Kinetic Monte Carlo algorithm (described later in Chapter 5). The x, y, $z$ positions of this cluster was put into a cell of a defined volume. This volume was now considered as a unit cell, with no pre-defined symmetry. This unit cell was sliced in the direction of the electron beam into ' $n$ ' number of slices. The software then calculated the diffraction pattern as the electron beam traversed through each of these slices, in effect giving the pattern that would be generated out of the 3-D crystal structure.

A software called 'Crystal Kit', also developed by Roar Killas, was used for structure factor calculations of crystals whose symmetry could be pre-defined. These structure factor calculations were used to obtain kinematic diffraction patterns. This exercise could also be performed manually using equation 3.1 , however obtaining the 
diffraction patterns at different zone axis can be a tedious task especially for compounds. Crystal Kit provides a convenient means for these calculations.

\subsubsection{X-ray Diffraction}

$\mathrm{X}$-ray diffraction (XRD) is one of the simplest and oldest techniques used for phase-identification. When a monochromatic beam of X-rays is passed through crystalline matter, diffraction occurs when Bragg's condition is satisfied. The relationship between wavelength of the X-ray, the interplanar spacing, $\mathrm{d}$, and angle between the transmitted and diffracted beam $2 \theta$ is given by Bragg's law,

$\lambda=2 \mathrm{~d} \sin \theta$

$\mathrm{XRD}$ is a non-destructive technique used for phase identification, compositional analysis, and crystal size determination. Crystal size ( $\mathrm{t}$ ) is determined by Scherrer formula [131],

$\mathrm{t}=(0.9 \lambda) /\left(\mathrm{B} \cos \theta_{B}\right)$

Where $\lambda$ is the wavelength of X-ray radiation, $\mathrm{B}$ is the angular full-width at half the maximum intensity and $\theta_{\mathrm{B}}$ is the Bragg's angle.

A film of carbon-nanocrystals synthesized was crushed and mounted on a plate. Phase identification was done using a Rigaku powder x-ray diffractometer (DMAXB). However, the disadvantage of XRD is that the information obtained is for the bulk sample and not for a highly localized region in the sample. For instance, when analyzing the carbon nanocrystalline film, it contains a large variety of phases; hence it will not be possible to assign peaks to particular regions on the sample. 


\subsubsection{Atomic Force Microscopy (AFM)}

AFM is a type of scanning-probe microscopes that can be used to obtain the topography of the sample. AFM was used to determine the crystallographic planes that bound the different diamond crystal morphologies by determining the angle between the planes. The AFM used in these studies was a Park Scientific Model M5 with a silicon tip operating in contact mode. To acquire an image, the microscope raster-scans a probe across the sample. In the "contact" mode, the instrument lightly touches a cantilever that holds the probe tip. As a raster-scan drags the tip over the sample, a piezoelectric detection apparatus measures the vertical deflection of the cantilever, which indicates the local sample height. Thus, in contact mode the AFM measures hard-sphere repulsion forces between the tip and sample. One of the major problems encountered in scanning diamond samples was that the tip used to break very easily. For more information on AFM, the reader is referred to [133].

\subsubsection{Raman Spectroscopy}

Raman measurements were performed using a Renishaw 1000 Raman microscope, with laser excitation wavelengths of $400-785 \mathrm{~nm}$. This non-destructive technique did not require any specific sample preparation. This technique was used to identify the $\mathrm{sp}^{2}$ and $\mathrm{sp}^{3}$ phases in the nanocrystalline carbon samples.

When a monochromatic radiation is impinged on a sample, partial scattering of the radiation occurs. Most of the scattered photons have the same wavelength as the incident photons, called Raleigh's scattering. However, very few photons ( 1 in every $10^{7}$ ) have wavelengths different from the incident ones and constitute the Raman 
scattering. Raman scattered photons are of a higher wavelength compared to the incident photons (Stokes shift), while a small number have a lower wavelength (antistokes shift). Along with a change in wavelength, the polarizability of light also changes.

Infrared spectroscopy (IR) is similar to Raman, in that it measures the molecular vibrations when impinged by photons. However, for a vibration to be IR active, the dipole-movement must also change in addition to the wavelength. Raman has several advantages over IR, one of them is no stringent requirement of large sample size, making it useful to analyze the nanocrystalline carbon samples in this work.

Raman spectroscopy instrumentation involves a laser (source of monochromatic radiation), a probe (that collects the non-Raleigh scattered radiation), and a spectrograph (that separates the scattered radiation by wavelengths and determines the intensity at each wavelength). In the Raman spectrum, the intensity of the scattered photons is plotted against the difference in frequency from the incident radiation. The $\mathrm{x}$-axis is generally called the Raman shift $\left(\mathrm{cm}^{-1}\right)$ and the $y$-axis is the intensity of the phonons. Since the difference in wavenumber is plotted, the position of the Raman shift is independent of incident laser wave length for most cases. However, there are a few exceptions to this rule, one being the D-band of graphite (discussed in detail in Chapter 7). Further details on the Raman technique can be found in reference [134].

\subsubsection{X-ray Photoelectron Spectroscopy (XPS)}

The surface functional group information can be obtained using XPS. When a surface is illuminated with photons of sufficient energy hv, the electrons of the surface atoms are excited from various energy levels to above vacuum level $E_{V A C}$. These 
electrons are called photoelectrons. The kinetic energy of the ejected photoelectrons is related to its binding energy in the atom by,

$\mathrm{h} v=\mathrm{E}_{\mathrm{B}}+\phi+\mathrm{KE}$

where, $h v$ is the incident photon energy, $E_{B}$ is the binding energy of the electron, $\phi$ is the work function and KE is the kinetic energy of the emitted photoelectrons. Hence, XPS measures the binding energy of the ejected photoelectrons when the sample is illuminated by X-rays of known wavelength.

Typically two X-ray sources are available, so that excitations at higher energy levels are possible: $\mathrm{Al} \mathrm{K \alpha}$ at $1486.6 \mathrm{eV}$, and $\mathrm{Mg} \mathrm{K} \alpha$ at $1253.6 \mathrm{eV}$. This X-ray source is sufficient to excite electrons from certain core levels. The kinetic energy of the photoelectrons emitted is determined by a concentric hemispherical analyzer. The core level binding energy can be deduced from kinetic energies of emitted photoelectrons, from which the parent atom can be identified. Thus, XPS is typically used for chemical analysis. XPS is sensitive to the top $10 \mathrm{~nm}$ of the surface. When an element is oxidized, for instance, some electrons from the outer orbital are shared with oxygen, resulting in a higher binding energy of the electrons in the oxidized atom. The change in electron binding energies due to change in chemical state is known as chemical shift, typically few $\mathrm{eV}$. Thus one can distinguish between the different chemical states of the atom, and get information about the surface functional groups [135].

The surface compositional analysis of the nanocrystalline carbon films was performed using V G Scientific x-ray photoelectron spectroscopy (XPS) using Al Ka xrays $(\lambda=8.34 \AA)$ output from aluminum and a spectrometer pass energy of $50 \mathrm{meV}$. The limitation of this technique (under the given experimental set-up) was the large spot-size 
of the X-ray beam. The small size of the samples deposited on the Pt tips necessitated bundling three wire-tips together to obtain adequate sampling signal. A fresh plasmacleaned $\mathrm{Si}(100)$ surface served as a support for the bundle, and a spectrum of identically treated $\mathrm{Si}(100)$ was used as a blank to account for carbon and/or oxygen contributions from the support. The $\mathrm{C}(1 \mathrm{~s})$ and $\mathrm{O}(1 \mathrm{~s})$ spectral windows from the blank were subtracted from the observed $\mathrm{C}(1 \mathrm{~s})$ and $\mathrm{O}(1 \mathrm{~s})$ features from the supported electrodes. In this way, the XPS data obtained were representative of only the nanocrystalline carbon surfaces.

\subsection{Electroanalytical characterization}

Electrochemical measurements made for analytical purposes, such as detecting a compound, is called electroanalysis. These experiments involve the combination of current, potential or charge and their relationship to the chemical parameters. Electroanalytical studies on the nanocrystalline carbon phases mainly involved potentiostatic measurements.

Potentiostatic measurements deal with study of charge-transfer (oxidation and reduction processes) at the electrode-solution interface based on dynamic situations. Potential is applied across the working electrode to carry out electron transfer reactions and the resulting current is measured. Thus, the current reflects the rate at which electrons move across the electrode-solution interface.

Carbon has been widely used for electrochemistry. Hence, the nanocrystalline carbon films synthesized were studied for their electrochemical properties. Two main types of studies were made, Cyclic Voltammetry (CV) (for studying its applicability for electron transfer reactions) and Electrochemical Impedance Spectroscopy (EIS) (for 
capacitance measurements). The experimental set-up uses a working electrode (the nanocrystalline carbon electrode), a reference electrode $(\mathrm{Ag} / \mathrm{AgCl}$ in $3 \mathrm{M} \mathrm{KCl}$ solution) and an auxiliary electrode (platinum wire/mesh) placed in an electrolyte solution of interest.

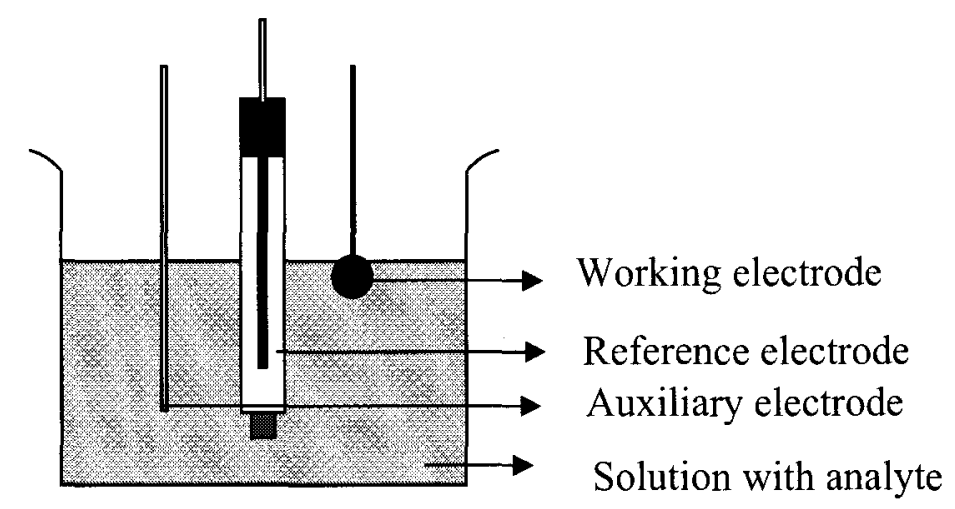

Figure 3.4 Illustration of a three-electrode electrochemical cell setup used for cyclic voltammetry.

\subsubsection{Cyclic voltammetry}

Cyclic Voltammetry is a potential sweep experiment in which the potential is scanned linearly across the working electrode. The working electrode, reference electrode and auxiliary electrode are in contact with a solution that has species, which get oxidized or reduced. The main purpose of this experiment was to obtain a current response from the carbon material that is related to the concentration of the target analyte.

Consider the following redox process occurring in a solution that contains the analyte $\mathrm{O}$ :

$\mathrm{O}+\mathrm{n} \cdot \mathrm{e}-\Leftrightarrow \mathrm{R}$ 
Where $O$ and $R$ are the oxidized and reduced species of the redox couple. Thermodynamically, the potential of an electrode is related to the concentration of the oxidized and reduced species by Nernst equation:

$$
E=E^{o}+\frac{2.3 R T}{n F} \log \frac{C_{O}(0, t)}{C_{R}(0, t)}
$$

Where $\mathrm{E}^{\mathrm{o}}$ is the standard potential for the redox reaction, $\mathrm{R}$ is the universal gas constant $\left(8.314 \mathrm{JK}^{-1} \mathrm{~mol}^{-1}\right)$, $\mathrm{T}$ is the Kelvin temperature, $\mathrm{n}$ is the number of electrons transferred in the reaction, and $\mathrm{F}$ is Faraday constant $(96487 \mathrm{C})$. Electron transfer reaction involves several steps, and the rate of the overall reaction is determined by the slowest (limiting) step. The main steps involved are mass transport of the electroactive species to the electrode surface, the electron transfer across the interface, the transport of the product back to the bulk solution and other processes like adsorption, desorption or electrodeposition. When a reaction is controlled solely by the rate at which the electroactive species reach the electrode surface, the current is said to be mass transportlimited. These reactions are called Nernstian or reversible reactions.

In a $\mathrm{CV}$, as the potential is scanned across the sample beyond the equilibrium potential $\left(\mathrm{E}^{\mathrm{o}}\right)$, oxidation occurs according to an anodic process where electrons are given up by the species. This process is characterized by a peak for oxidation $\left(E^{\mathrm{a}}\right.$ or $\left.E^{\mathrm{ox}}\right)$. Similarly when the potential reaches below the equilibrium potential, a reduction process occurs according to a cathodic process where electrons are accepted by the species. This process is characterized by another peak $\left(E^{c}\right.$ or $\left.E^{r c d}\right)$. The current produced during oxidation or reduction (at $\mathrm{E}^{\mathrm{ox}}$ or $\mathrm{E}^{\mathrm{rcd}}$ ) is in the form of a peak shape, due to the diffusion limitation. Multiple peaks are produced with multiple electron exchange reactions occurring for multiple species. The difference between the $\mathrm{E}^{\mathrm{ox}}$ and $\mathrm{E}^{\mathrm{rcd}}$ is called $\Delta \mathrm{E}_{\mathrm{p}}$, 
which is an important parameter. Ideally, for a simple reversible electron exchange such as equation 3.6, $\Delta \mathrm{Ep}$ is given by $0.059 / \mathrm{n} \mathrm{V}$. This is a criterion from Nernstian behavior. For the reactions where electron transfer is sluggish, the oxidation/reduction peak-sizes are reduced and widely separated, and hence $\Delta \mathrm{Ep}$ is different from $0.059 / \mathrm{n} \mathrm{V}$. More information on cyclic voltammograms can be obtained from references [136] and [137].

The purpose of doing CVs was to measure this difference in potential, $\Delta \mathrm{E}_{\mathrm{p}}$ for the electron transfer for different biologically important analytes. The $\Delta \mathrm{E}_{\mathrm{p}}$ is different at different carbon surfaces, graphite edge-planes, basal-planes and diamond. Thus, CVs helped in understanding the types of sites present at the surfaces of the crystalline carbon films. Also, CVs helped in determining the applicability of carbon nanocrystalline materials for in vivo electroanalytical sensing.

Cyclic voltammetry (CV) experiments were carried out with a Bioanalytical Systems Model CV-50W Voltammetric Analyzer equipped with a single-compartment, three-electrode cell with $\mathrm{Ag} / \mathrm{AgCl}(3 \mathrm{M} \mathrm{KCl})$ reference and $\mathrm{Pt}$ wire auxiliary electrodes. The area (geometrical and electrochemical) varied with each electrode and was determined approximately. In some CV experiments, glassy carbon and B-doped microcrystalline diamond (MCD) electrodes were employed for comparison purposes. GC electrodes were obtained from Bioanalytical Systems, while MCD electrodes were synthesized as described in section 3.2. CVs of the nanocomposite carbon deposits were obtained by immersing the head of the electrode (ball-shaped) into the solution and blanking the rest of the electrode with an insulating enamel to ensure the electrochemical response originated only from the nanocomposite material. Most of the analytes, serotonin (5-hydroxytyramine), uric acid, ascorbic acid, dopamine, potassium 
ferricyanide $\left(\mathrm{K}_{3}\left(\mathrm{Fe}(\mathrm{CN})_{6}\right)\right)$, catechol, 4-methyl catechol, ruthenium hexamine hydrochloride, etc. were obtained from Sigma-Aldrich. CVs were usually run in $0.1 \mathrm{M}$ $\mathrm{KCl}$ electrolyte, but a $0.2 \mathrm{M}$ phosphate buffer solution was used occasionally to maintain a pH of 7. De-aerating the solutions had little effect on the observed CVs.

\subsubsection{Electrochemical impedance spectroscopy (EIS)}

The theory of electrode double layer was developed by Helmholtz in 1850 . According to this theory, an electrode surface is assumed to have an excess charge. In order to keep the surface neutral, the electrode attracts ions of opposite charge. The charges on the electrode surface are separated from the ions by a small distance in the order of $\AA$. This scenario is similar to a capacitor. However, the outer layer of ions has two layers, one called the inner Helmholtz plane (IHP) and the outer region is called the outer Helmholtz plane (OHP). IHP is formed by ions that are adsorbed on the surface, while OHP represents the solvated ions at the closest distance to the surface. Hence, the surface behaves like a double layer capacitor. This double layer capacitance exists even when an electrode is taken out from the solution.

Impedance analysis is used to measure the double layer capacitance of the electrode surface. Electrochemical impedances are measured by applying an AC potential to an electrochemical cell and measuring the current through the cell. The equivalent circuit model of an electrochemical cell is given by Figure 3.5 [138]. As shown in the figure, the total current through the working interface is a sum of the distinct contributions from faradaic processes $\left(i_{f}\right)$ and double layer charging $\left(i_{c}\right)$. The double layer capacitance is a pure capacitance, and hence is represented as C. The 
faradaic processes, in the case of a simple irreversible reaction, can be considered as a pure resistance independent of frequency, and is called $R_{c t}$ (charge transfer resistance). All the current must pass through the solution resistance; therefore $R_{\Omega 2}$ is inserted as a series element to represent this effect in the equivalent circuit. EIS measures the way the total impedance $\mathrm{Z}$ changes with frequency.

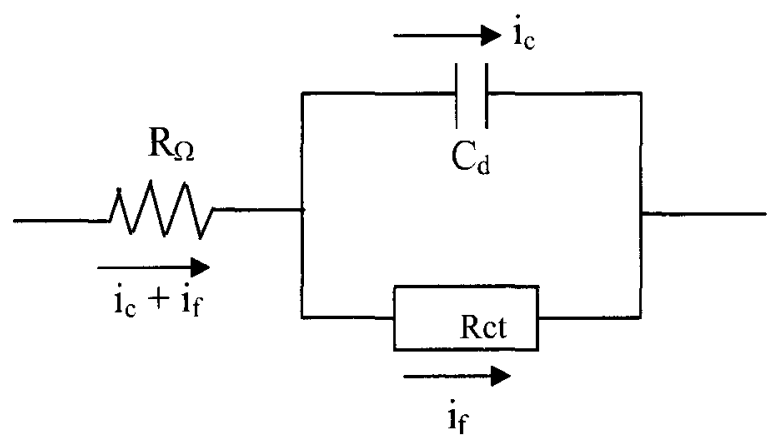

Figure 3.5 Equivalent circuit model of an electrochemical cell.

The solution to this equivalent circuit model, yields the total impedance (sum of resistances when in series as in Figure 3.5) being equal to $R_{\Omega}+R c t$ at low frequencies and $R_{\Omega}$ at very high frequencies. Hence, the logarithmic plot of total impedance versus frequency exhibits a slope of -1 , with an intercept equal to $1 / C_{d}$ at $\omega=1$ radians. This logarithmic plot is called Bode plot, which is used to determine $\mathrm{R}_{\Omega}$ and $\mathrm{C}_{\mathrm{d}}$. One could also use the Nyquist plot for determining $C_{d}$. Electrode Impedance Spectroscopy (EIS) was performed with a 273A Princeton Applied Research (PARC) Potentiostat/Galvanostat in $0.1 \mathrm{M} \mathrm{KCl}$ solution. The frequency range used was 11.31 $\mathrm{mHz}$ to $100 \mathrm{kHz}$. 


\subsection{Summary}

This chapter provided the description of the synthesis and characterization tools used in this work. Due to the nanometer scale of the carbon materials studied in this work, a variety of materials characterization tools were used to yield complementary information for identifying their structure and morphology. 


\section{CHAPTER 4}

\section{STRUCTURES OF CARBON NANOCRYSTALS}

\subsection{Introduction}

Carbon deposition from the vapor phase under most conditions of low pressure chemical vapor deposition (Figure 1.1) must result only in graphitic phases according to thermodynamics. However, the fact that diamond (a metastable phase) occurs at these conditions indicates that it is possible to nucleate carbon into various thermodynamically unfavorable phases. Once a phase is nucleated (achieves a stable cluster size), it can continue to grow without hindrance. Thus, nucleation is the critical step in synthesizing metastable phases such as diamond.

Although few reports claimed homogenous nucleation of diamond $[50,139,140]$, they could not account for the large nucleation densities observed on substrates prepared with different pre-treatments. Hence, diamond nucleation is believed to occur primarily by heterogeneous nucleation on the substrate surface. At the same time, classical nucleation theory is not applicable in predicting the critical nuclei size and structure for heterogeneous nucleation. High-energy sites on the surface play an important role in diamond nucleation on non-diamond substrates [141]. Thus, there has been a continuous effort to study diamond nucleation by capturing the initial stages of diamond crystal formation. 
Over the years, there have been several hypotheses regarding the mechanism of diamond nucleation from the vapor phase, many of which involve diamond nuclei formation via intermediate phases. Angus et al. [52] suggested that diamond nucleation could initiate by hydrogenation at the edge-planes of graphite followed by the formation of a nucleus containing parallel stacking errors. Further growth of this nucleus would give rise to a crystal with a hexagonal-diamond core (also called lonsdaleite) core at nanoscale. Lifshitz et al. [55] proposed that diamond nucleation occurs from hydrogenated amorphous carbon nanoclusters. Both these theories concluded diamond nucleation involves imperfect diamond intermediates.

Interestingly, some new phases of carbon with fcc structure were reported at nanoscale during the shock compression of graphite [16-20]. Later, these same phases were also observed during chemical vapor deposition of diamond [21-25]. The fcc-phase of carbon, with the same lattice parameter as diamond, was termed 'n-diamond' and with lattice parameter greater than diamond was termed ' $\mathrm{i}$-carbon'. The evidence claiming the existence of these new phases for carbon is only by using diffraction analysis. The structure of carbon in the fcc phase or its relation to the well-understood cubic phase of diamond is not yet known due to the limited quantity of these phases synthesized and the limited study to identify the structure of these phases. Thus, one of the possible ways of investigating the path of diamond nucleation would be to capture a large quantity of diamond nuclei (nanocrystals of carbon), and study the structure of the intermediate phases formed. This study could give an indication to the path of diamond nucleation as well as lead to possible discovery of new phases for carbon at nanoscale. 
This chapter discusses the extensive investigations carried out to synthesize a large density of carbon nanocrystals $(2-10 \mathrm{~nm})$ from the vapor phase and study their structure using electron nanodiffraction. The nanodiffraction measurements of the observed cubic carbon phases are complimented with theoretical studies to identify their structure. Finally, a possible link between these new phases of carbon and diamond nucleation is also discussed.

\subsection{Results from nucleation experiments}

As described in Chapter 3, the platinum wire substrates placed directly in the dense zone of the microwave plasma, under diamond growing conditions $\left(1-2 \% \mathrm{CH}_{4} / \mathrm{H}_{2}\right)$, get coated with a dense carbon deposit at the tip. As soon as the platinum wire was immersed into the plasma, it's tip formed into a ball due to the melting of platinum. Hence the temperature seemed to be highest at the tip of the platinum wire, close to the melting point of platinum $\sim 1768^{\circ} \mathrm{C}$.

Prior in situ plasma diagnostics have indicated that the temperature at the center of the plasma discharge is maximum (about $2000 \mathrm{~K}$ ) and changes by $1000 \mathrm{~K}$ with $1 \mathrm{~cm}$ change in radial position [82, 142]. Spatial profiles of temperature and neutral radical mole fractions were simulated in microwave plasmas at nearly the same gas phase conditions used in this work [143]. According to the gas phase compositions predicted, the radical $\mathrm{CH}_{3}$ is about an order of magnitude higher, and $\mathrm{CH}, \mathrm{CH}_{2}, \mathrm{C}_{2} \mathrm{H}$, are about two orders of magnitude higher at the center of the plasma discharge than at the substrate surface, which is about $2 \mathrm{~cm}$ away. $\mathrm{C}_{2} \mathrm{H}_{2}$, on the other hand, seems relatively less sensitive to the position in the plasma. Thus, at the center of the plasma, one can assume 
that there is high gas-phase supersaturation of carbon, leading to a high tendency of nucleating crystalline phases of carbon. On the other hand, reports have indicated that the gas composition close to the substrate is insensitive to the substrate temperature, i.e. by simply increasing the substrate temperatures one cannot really achieve a high density of gas phase radicals and hence increase nucleation [80]. Thus, the method of capturing high density of nuclei by immersing substrates into the dense zone of the plasma is quite novel.

Equilibrium gas phase calculations at high temperatures predict that the precipitated phase would be mostly non-diamond [144]. However, the plasma and radical species composition is far from equilibrium, and one could expect that reaction kinetics may favor nucleation of metastable phases including diamond.

As expected, a thick carbon deposit was obtained after about few hours of growth at the tip of the Platinum wires as shown in Figure 4.1. This deposit, which from here on will be referred to as ball-shaped deposit, was crushed and used for analysis by singlecrystal nanodiffraction. The crystal sizes typically observed was about $2-10 \mathrm{~nm}$.

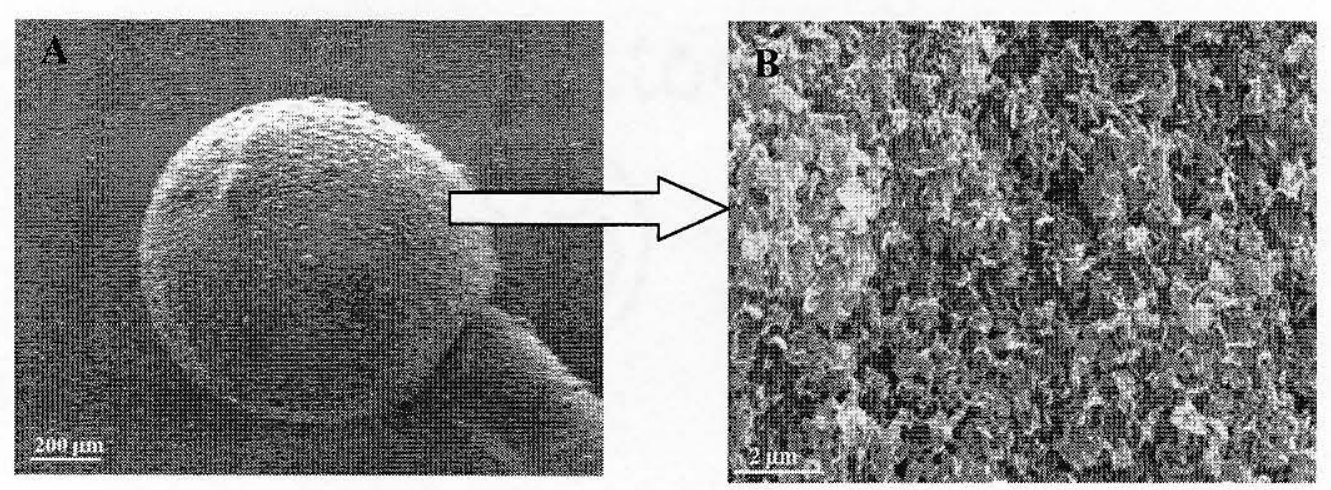

Figure 4.1 SEM image of the thick carbon deposit at the tip of a platinum wire obtained after deposition at (a) low magnification (500 X) and (b) high magnification (10k X). 
One $\mathrm{cm}$ away from the tip of the wire, clear facets of microcrystalline diamond were observed. However, these micro-diamond crystals were not a subject of investigation for crystalline structure investigations.

\subsection{Structures of carbon crystals analyzed by diffraction}

Nanodiffraction analysis was done using a scanning transmission electron microscopy (STEM) instrument, in which the incident beam of $100 \mathrm{keV}$ electrons was focused to a diameter of less than $1 \mathrm{~nm}$ at the specimen level [145]. As described in Chapter 3, this technique is capable of producing diffraction patterns from individual nanocrystals instead of obtaining diffraction patterns from a cluster of crystals like selected area diffraction. The diffraction patterns came from crystals in arbitrary orientations. So, it was not feasible to tilt the nanocrystals into axial orientations, so that one had to accept the patterns that appeared as the incident beam was moved over the specimen. For most beam positions, the patterns showed only a few widely spaced spots, which could not be interpreted in terms of any recognizable phase and were not recorded. Those patterns showing regular two-dimensional arrays of spots were recorded. About a hundred crystals in different zone axes were individually analyzed for their diffraction patterns. As described earlier, once a spot pattern is obtained, based on the ratio of distances from the center spot, the crystal symmetry can be easily indexed. The real distances between spots were determined after accounting for the magnification in the camera lenses, and this information was to estimate the lattice parameter of the nanocrystal. 
Two-thirds of the crystals observed exhibited diffraction patterns corresponding to bulk graphite. In all these crystals, for orientations almost perpendicular to the c-axis, the strong rows of spots corresponding to the c-spacing of $0.67 \mathrm{~nm}$ were prominent. With the beam parallel to the c-axis, the hexagonal spot pattern of graphite was readily identified by the characteristic intensity distribution of the (10.0) spots being much weaker than the (11.0) spots. These graphite crystals will be discussed in more detail in Chapter 7. The other one-third was indexed as cubic phases of carbon.

It was observed that nearly $80 \%$ of the indexed non-graphite carbon crystals had either the face-centered, body-centered, or hexagonal structure, very different from the perfect cubic-diamond structure. As mentioned in Chapter 2, the fcc phase of carbon with a lattice parameter of $0.356 \mathrm{~nm}$ has been reported before. This fec phase is termed as "n-diamond" [19]. Another modification of this phase termed as "i-carbon" exhibited lattice parameter greater than $0.356 \mathrm{~nm}$. The hexagonal structured diamond was called "lonsdaleite". There have not been any prior reports on the observation of a bcc structure for carbon.

TEM/EDS did not indicate the presence of any other element, other than carbon. More reliable results were obtained by attempting to detect heavier elements using the lattice parameters calculated by diffraction analysis for the crystals and eliminating the possibility of heavier element contamination.

Diffraction spots in nanodiffraction patterns typically tend to be rather large and often of irregular shape (see Figure 4.2). This, combined with the errors associated with the calibration of the instrument, gave an accuracy for the determination of lattice spacing 
that was rarely better than a few percent. This limited accuracy was sufficient, however, to distinguish the various phases.

Of the single-crystal diffraction patterns from other phases, the most common patterns were as follows:

(1) The typical pattern from fcc carbon in [110] orientation with strong (111) and (200)-type spots, is presented in Figure 4.2a. Crystals showing this diffraction pattern, and having a corresponding unit cell dimension $\mathrm{a}=0.36 \mathrm{~nm}$ were attributable to $\mathrm{n}$ diamond. Some with $\mathrm{a}=0.42 \mathrm{~nm}$ and with a similar diffraction pattern, could come from i-carbon. The plot in Figure 4.2b shows the numbers of such patterns recorded for the various a-values.
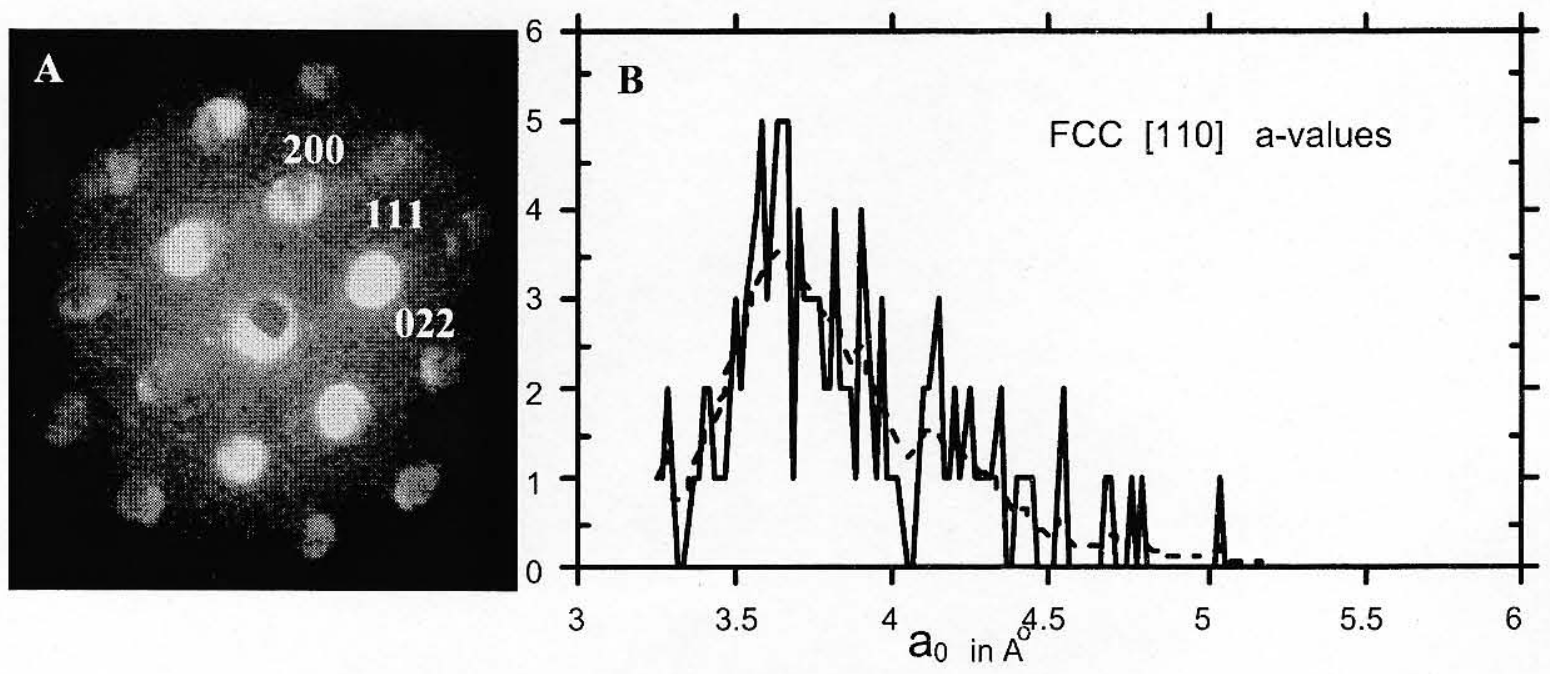

Figure 4.2 (a) Diffraction pattern of fcc carbon crystals in the [110] zone axis (b) Distribution of lattice parameters $\left(a_{0}\right)$ of crystals exhibiting pattern (a).

(2) Square patterns of spots such as that in Figure 4.3a, could come from a cubic fcc or bcc structures in [100] orientation. The plot in Figure 4.3b shows the numbers of these patterns recorded for various a-values, on the assumption of an fcc lattice. 
Figure $4.3 \mathrm{c}$ shows the distribution on the assumption of a bec lattice.

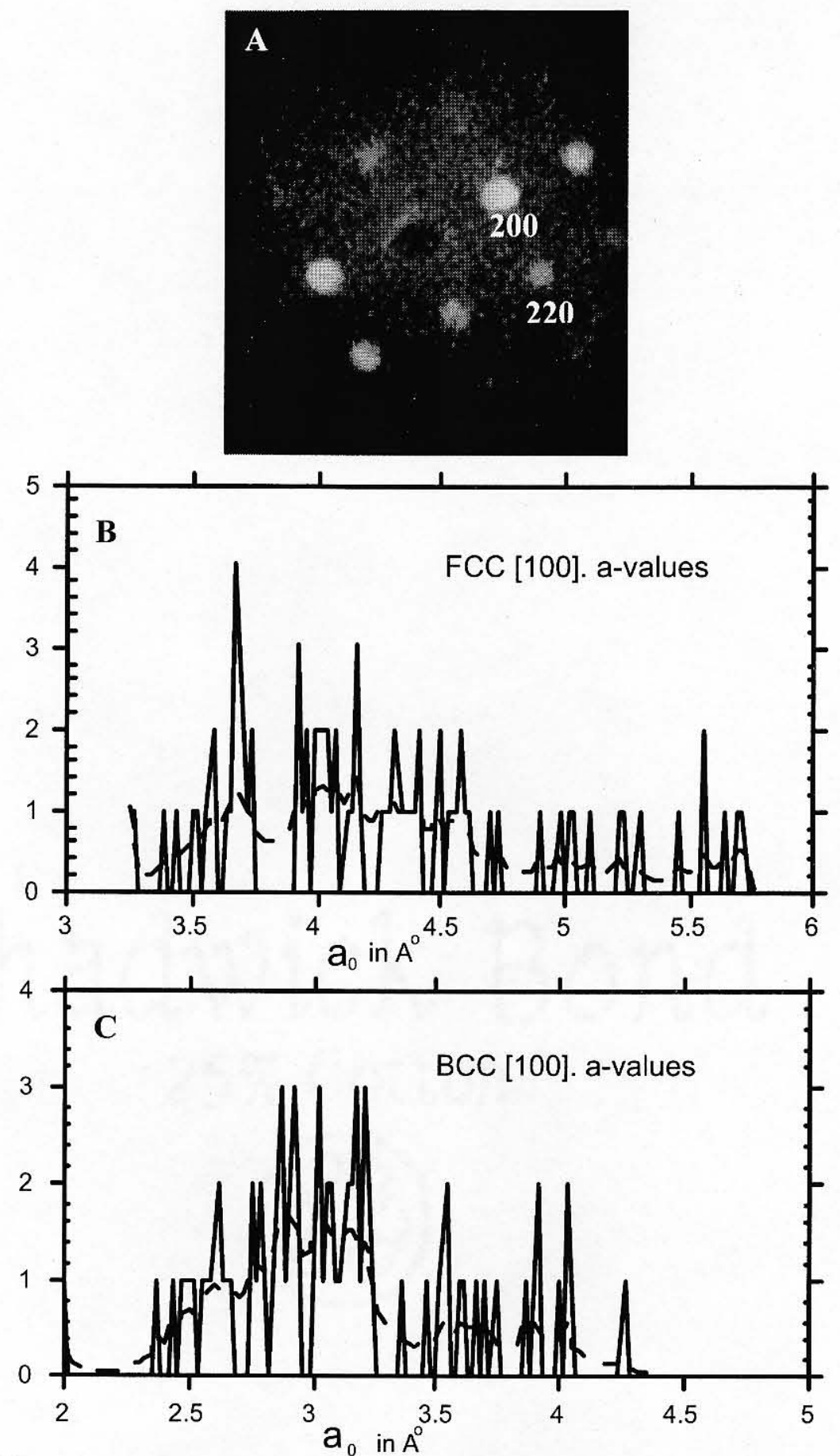

Figure 4.3(a) Diffraction pattern corresponding to [100] zone axis of a cubic lattice.

Distribution of lattice parameter of crystals exhibiting patterns (a), when indexed as: (b) fcc and (c) bcc. 
In Figure $4.3 \mathrm{~b}$ the patterns with lattice parameters close to $0.36 \mathrm{~nm}$ could come from an fcc lattice (n-diamond). But this peak also corresponds to the peak in Figure 4.3c for a bcc lattice with $\mathrm{a}=0.25 \mathrm{~nm}$. The group of patterns with approximately $\mathrm{a}=0.42 \mathrm{~nm}$ in Figure $4.3 \mathrm{~b}$ could come from i-carbon. This peak also corresponds to the peak in Figure 4.4 for a bcc cell with $\mathbf{a}=0.3 \mathrm{~nm}$. There is a small group of patterns in Figure $4.3 \mathrm{~b}$ with dimensions corresponding to fcc with about $0.39 \mathrm{~nm}$, which we assume to be close to the ones for $\mathrm{i}$-carbon at $0.42 \mathrm{~nm}$. Others, with greater a-values such as a $=0.55$ nm may come from larger fcc crystals described before [26-28]. These values still correspond to the peaks coming from bcc of lattice parameter $\mathrm{a}=0.35$ and $0.39 \mathrm{~nm}$ as shown in Figure $4.3 \mathrm{c}$. The main peak at about $0.31 \mathrm{~nm}$ in Figure $4.3 \mathrm{c}$ can be attributed to a bcc structure only, because of its presence in Figure 4.4b, and 4.5c.

(3) A rectangular diffraction pattern, with principal dimensions in the ratio of the square root of 2 is shown in Figure 4.4a. This can be attributed to a bec structure with $\mathrm{a}=$ $0.31 \mathrm{~nm}$, approximately. The distribution of unit cell a-values for bcc structures in [110] orientation is shown in Figure 4.4b. 

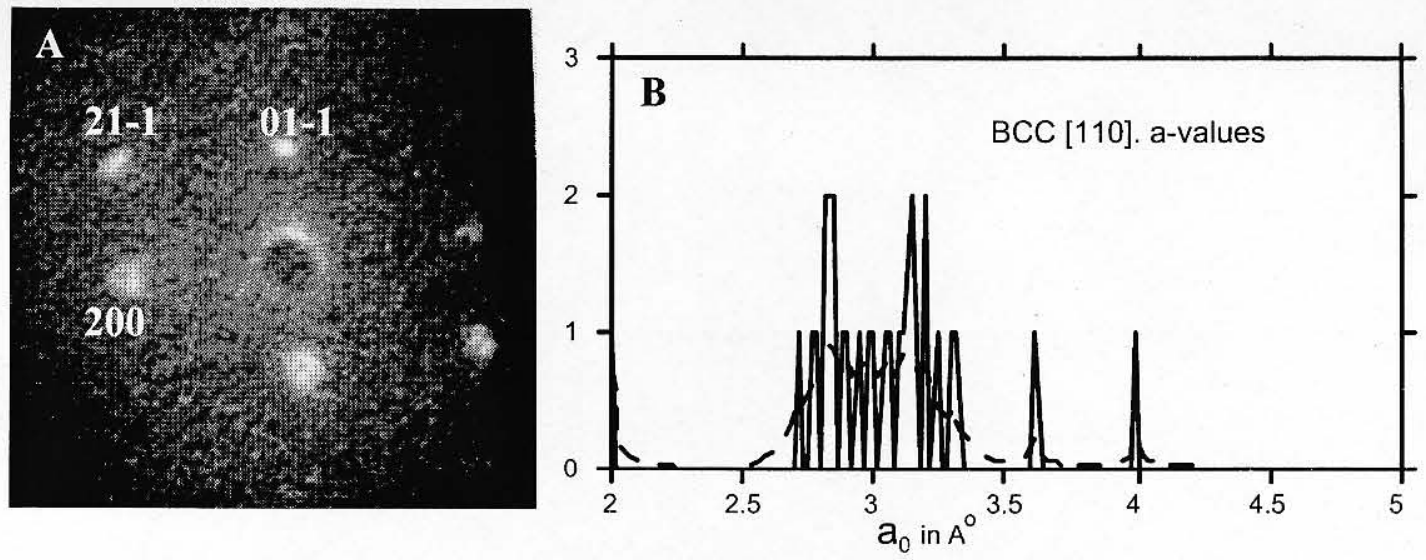

Figure 4.4(a) Rectangular diffraction pattern of bec carbon crystals in the [110] zone axis.

(b) Distribution of lattice parameters of crystals exhibiting pattern (a).

(4) In the hexagonal patterns of Figure 4.5a, the (100) and (110) spots are of comparable intensities, which is distinct from the hexagonal patterns from graphite. The observed distribution of lattice parameters, a-values for a hexagonal cell, is shown in Figure 4.5b. Such patterns could also be attributed to cubic unit cells in [111] orientation. If they are assigned to a bcc cell in [111] orientation, the distribution of observed a-values is as shown in Figure $4.5 \mathrm{c}$. The main peak of Figure $4.5 \mathrm{c}$, at about $0.3 \mathrm{~nm}$, corresponds to the main peak of Figure $4.5 \mathrm{~b}$, at about $0.252 \mathrm{~nm}$ (lattice parameter for hexagonal diamond). Hence, there is an uncertainty as to the relative amounts of the bcc structure in [111] orientation and the hexagonal structure in $[00,1]$ orientation. It would be expected that the number of patterns produced with beam directions approximately perpendicular to the c-axis, would be comparable to the number of patterns parallel to the $\mathrm{c}$-axis for hexagonal diamond $(a=0.252 \mathrm{~nm})$. However, there were very few patterns of hexagonal diamond with c-axis perpendicular to the beam direction. Hence it is suggested that most of the main peak in Figure 4.5b comes from bcc in [111] orientation. 

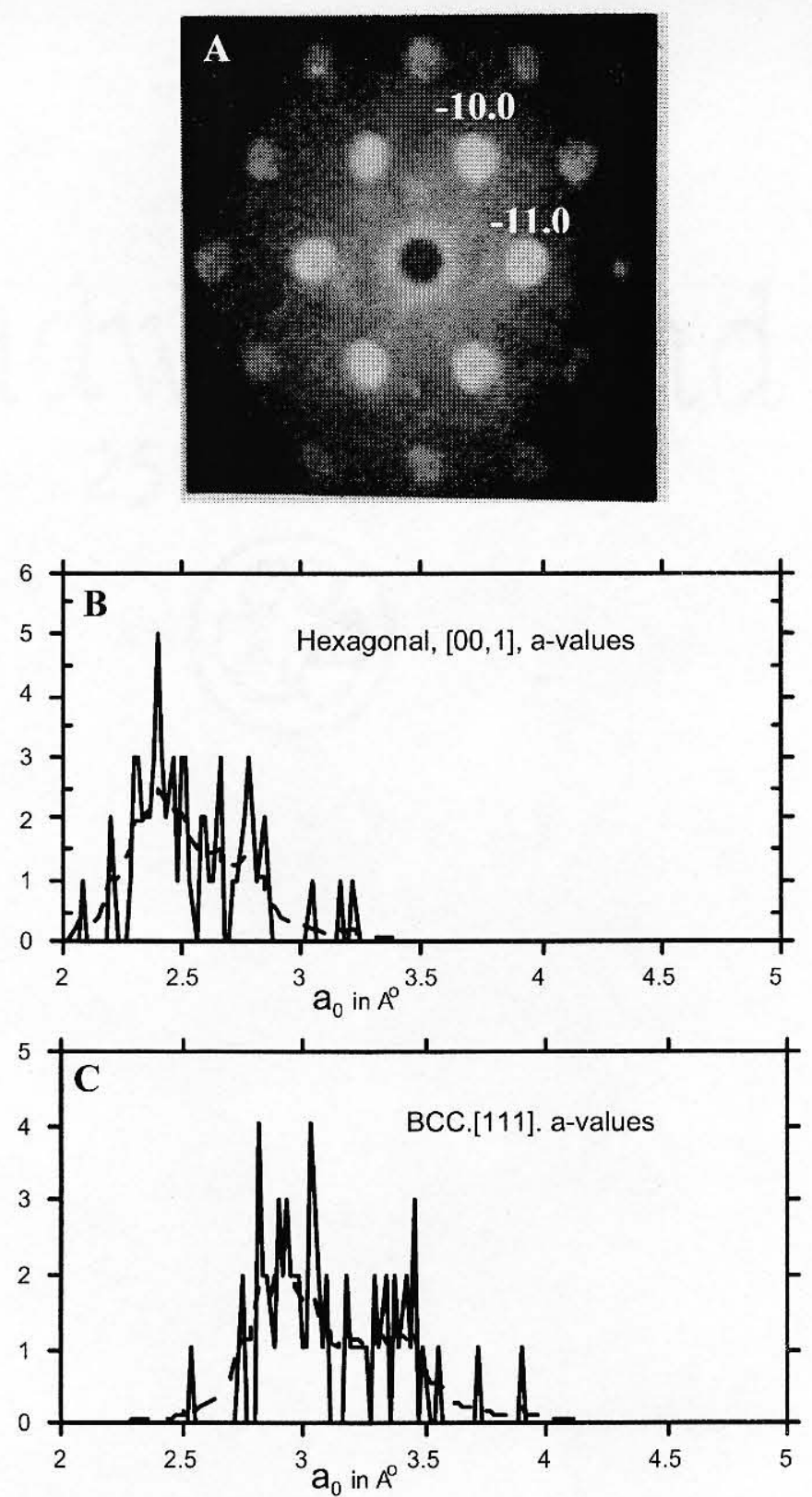

Figure 4.5(a) Hexagonal diffraction pattern corresponding to lonsdaleite in [001] zone axis or bcc in [111] zone axis. Distribution of lattice parameters of crystals exhibiting patterns (a), when indexed as (b) lonsdaleite and (c) bcc. 
The appearance of a subsidiary peak at about $0.34 \mathrm{~nm}$ in Figure $4.5 \mathrm{~b}$ and the equivalent $0.28 \mathrm{~nm}$ in Figure $4.5 \mathrm{c}$ could not be interpreted. This is also equivalent to the $0.35 \mathrm{~nm}$ peak visible in Figure 4.3c but only weakly present in Figure 4.4b.

The main conclusions that can be drawn from this data on lattice dimensions is that the most common phase present is the fcc $n$-diamond $(a=0.36 \mathrm{~nm})$. Other phases include the i-carbon (again, an fcc phase with a lattice parameter of $0.42 \mathrm{~nm}$ ), a bcc phase with cell dimension approximately $0.31 \mathrm{~nm}$, a small amount of hexagonal diamond, and possible small amounts of other phases.

\subsection{Understanding the n-diamond (fce) structure by electron diffraction simulations}

The n-diamond phase appears to have a simple fcc structure, as suggested by the presence of the (200), (222) and (420) reflections, which are forbidden for normal diamond. Hence, it has the $\mathrm{Fm} \overline{3} \mathrm{~m}$ symmetry of an fcc lattice instead of a $\mathrm{Fd} \overline{3} \mathrm{~m}$ of a diamond cubic lattice. The implication is that there are only 4 carbon atoms in the unit cell instead of the 8 atoms for diamond. The difference in these two structures and their respective diffraction patterns is shown in Figure 4.6 

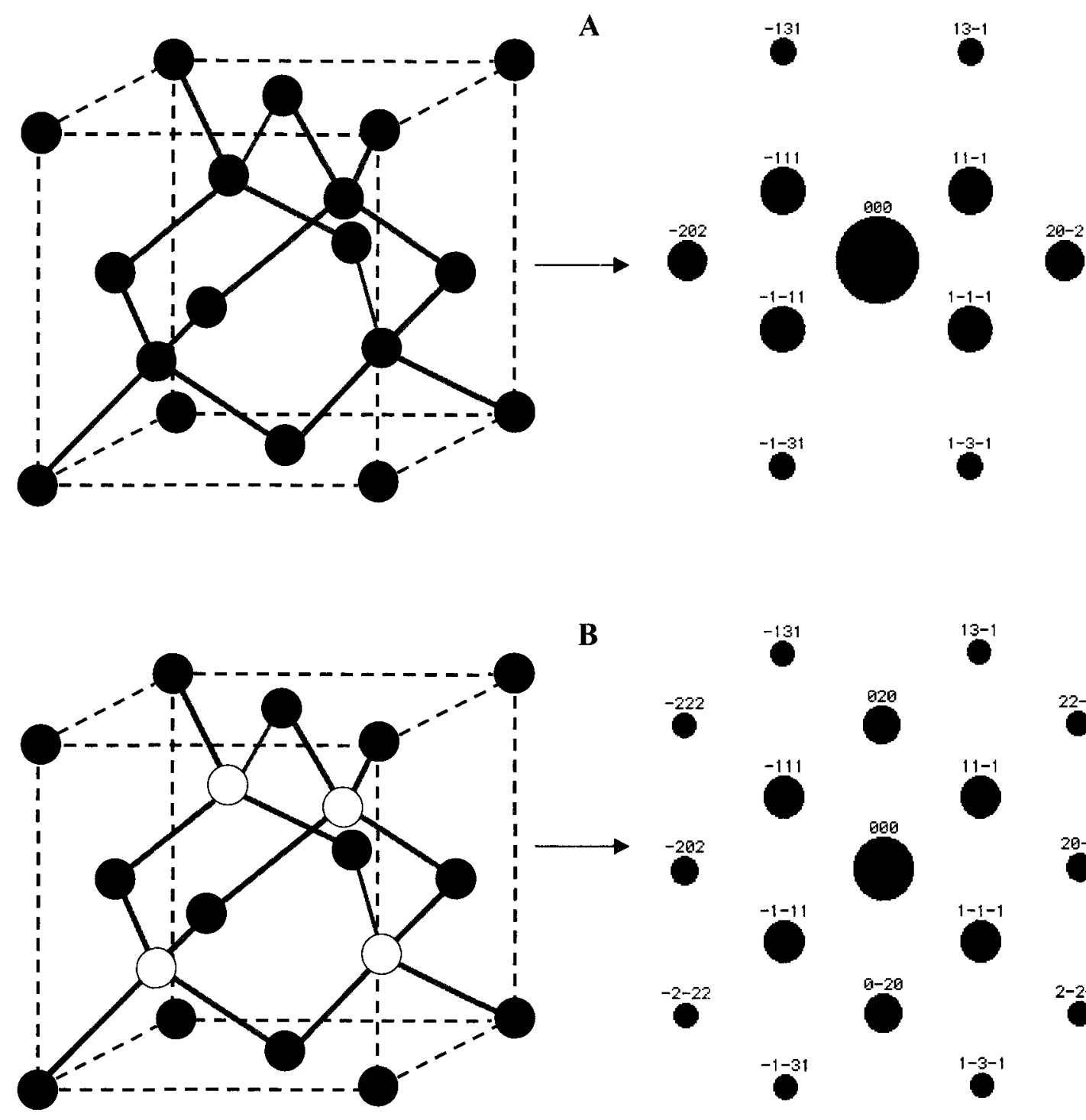

B
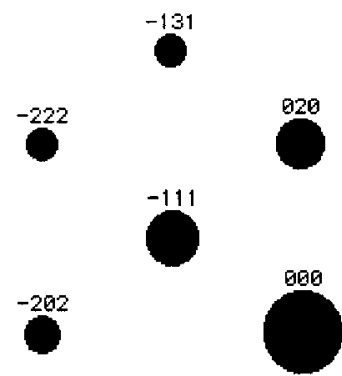

18-1
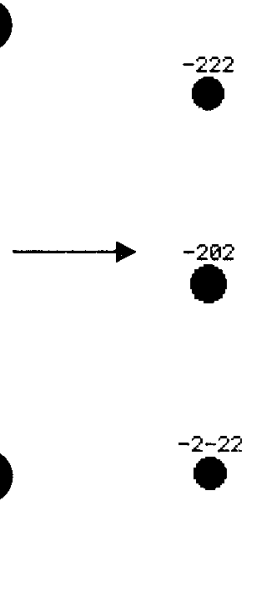

$-1-11$
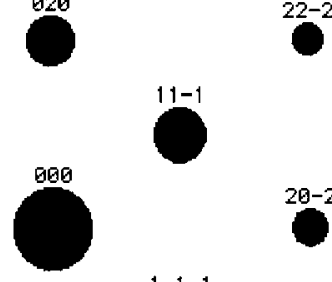

$0^{20-2}$
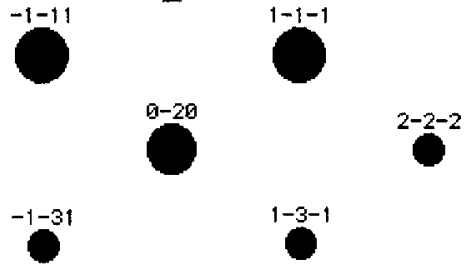

$\mathbf{Q}^{1-3-1}$

Figure 4.6 (a) The crystal structure of diamond $(\mathrm{Fd} \overline{3} \mathrm{~m})$ and corresponding diffraction pattern in the [110] zone axis. (b) The crystal structure of fcc $(\mathrm{Fm} \overline{3} \mathrm{~m})$ and corresponding diffraction pattern in [110] zone axis.

There can be several possible reasons for the observations of a diffraction pattern that do not correspond to a usual expected pattern: (1) contamination of the sample with 
another element (2) spurious diffraction patterns due to large thickness of the sample, often called double-diffraction (3) changes/defects in the crystal structure (4) a new phase Diffraction patterns taken from these samples did not indicate any large lattice parameters that could be attributable to platinum phases. TEM/EDS performed to check for contamination of the samples also confirmed the absence of any other element other than carbon, within the limits of elements detectable by EDS.

It is well known that the intensities of electron diffraction single-crystal patterns may be strongly affected by dynamical scattering except for very small crystal thicknesses with only light atoms. This phenomenon is called double-diffraction [129]. Calculations of intensities for diamond crystals of increasing thickness were made using the multislice programs for dynamical scattering using the software MacTempas (developed by Roar Kilaas, NCEM, CA). The crystal size and beam voltage assumed for these calculations were $50 \mathrm{~nm}$ and $200 \mathrm{keV}$. In Figure 4.7a the calculated intensities of the (200) and (111) for the diamond structure are plotted against thickness for the [110] orientation and in Figure 4.7b, a similar plot is given for the [100] orientation. It is evident from Figure 4.7a that for the [110] orientation the forbidden (200) reflection may be produced by dynamical scattering effects. Referring back to Figure 4.6a, the 111 beam acts like a new incident beam and is re-diffracted by the (111) plane. Thus the result is that the $(200)$ reflections appear: $(11-1)+(1-11)=(200)$. However, from Figure 4.7a the (200) intensity is very small compared with the dominant (111) reflection for a crystal thickness of less than $10 \mathrm{~nm}$. The crystals that were observed experimentally were of the size of $2-10 \mathrm{~nm}$. For the [100] orientation, the (200) reflection never gains appreciable intensity for diamond. The reason for this is that unlike the [110] ZA, no two 
vectors add up to give the (200) in the [100] ZA. The fact that the (200) was observed in the [100] ZA patterns indicated that they must be coming from an fcc phase. Hence, these calculations concluded that the forbidden spots of the n-diamond phase were not due to spurious diffraction effects.

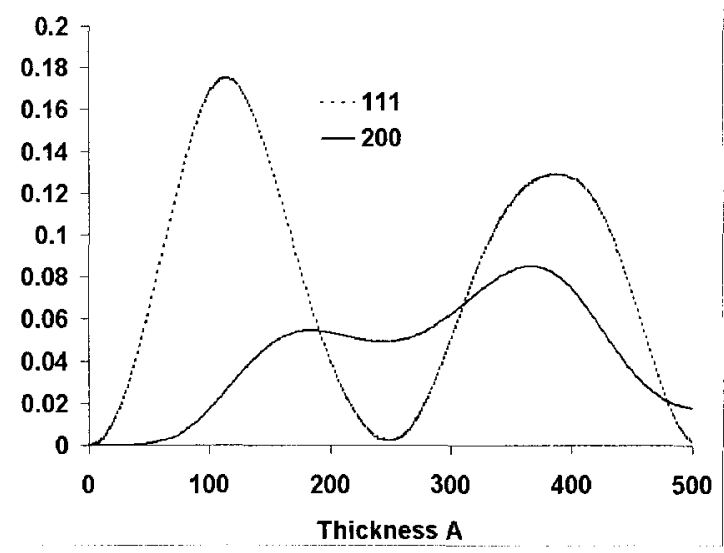

A

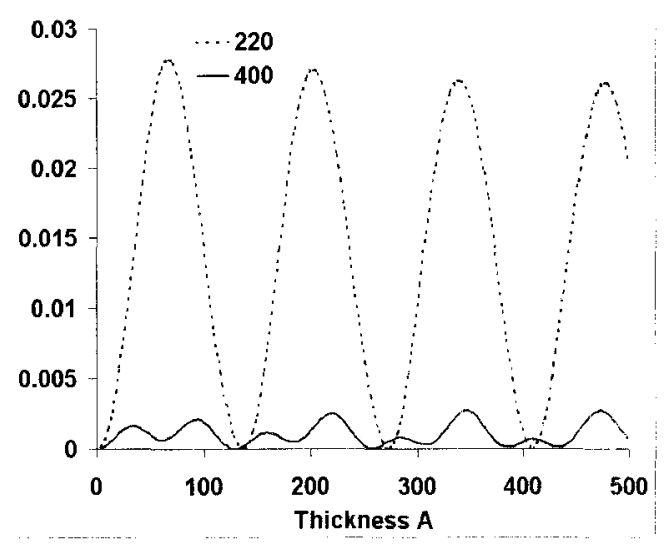

B

Figure 4.7 Dynamical diffraction calculations for variation of intensities of spots with diamond crystal thickness in the (a) [110] and (b) [100] zone axis.

Attempts were made to eliminate the possibility of defects in the diamond structure as being responsible for observing diffraction patterns that are forbidden for perfect diamond structure. Several simulations were carried out to grow clusters with defects such as mixture of $\mathrm{sp}^{2}$ and $\mathrm{sp}^{3}$ phases, vacancies, stacking faults at the surface, etc. These clusters (typically $2 \mathrm{~nm}$ in size) were simulated using a Monte Carlo code (described in Chapter 5), that was further used for crystal morphology simulations.

A number of studies reporting the fcc phase of carbon, specially the n-diamond, saw this new phase along with a large amount of graphite, similar to this study. Hence, the possibility of a mixed phase of $\mathrm{sp}^{2}$ and $\mathrm{sp}^{3}$ could have been responsible for the 
observation of forbidden reflections in the diffraction patterns, corresponding to $\mathrm{n}$ diamond. Figure $4.8 \mathrm{a}$ shows the structure of a mixture of $\mathrm{sp}^{2}$ and $\mathrm{sp}^{3}$ phase that was simulated using KMC. As seen in the figure, the core of the cluster has a diamond structure ( $\mathrm{sp}^{3}$ bonding), while the surface is covered by large amounts of $\mathrm{sp}^{2}$ bonding. A similar thermodynamically stable nanocrystal was proposed, called bucky diamond [146] (Figure 4.8b). Simulation of the diffraction pattern from both these clusters gave. a diffraction pattern similar to cubic-diamond.

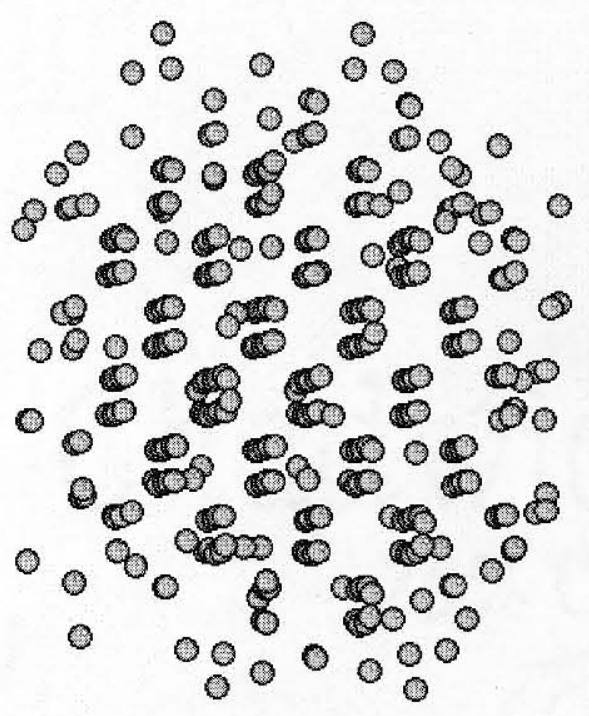

A

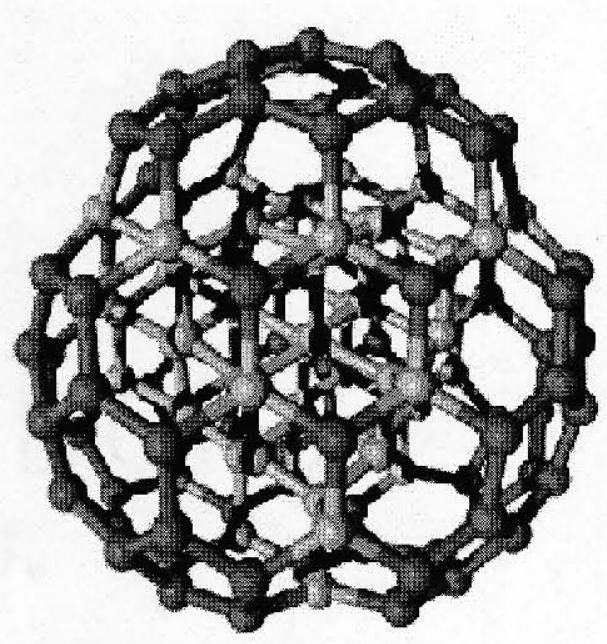

B

Figure 4.8 Model of simulated clusters (a) with $\mathrm{sp}^{3}$ core and $\mathrm{sp}^{2}$ bonds on the surface (b) bucky-diamond from Ref 146.

Figure 4.9a shows a simulated crystal with large amount of stacking faults at the surface. Stacking faults lead to multiply-twinned diamond crystals, which are commonly observed for diamond. This crystal was simulated by performing hexagonal step nucleation on $\{111\}$ planes of a growing diamond cluster with a finite probability. 
Figure $4.9 \mathrm{~b}$ shows a diamond structure with nearly $30 \%$ vacancies. This cluster was simulated by removing atoms at random from a diamond cluster, such that there are no atoms that are isolated in the crystal. Both of these extreme defective structures also did not produce the forbidden $(002)$ in the simulated diffraction patterns. It seemed that the presence of these defects did not break the symmetry of the diamond crystal structure.

Hence, these studies concluded that clearly double-diffraction, or defects in the diamond crystal structures are not responsible for observing diffraction patterns corresponding to these new phases.
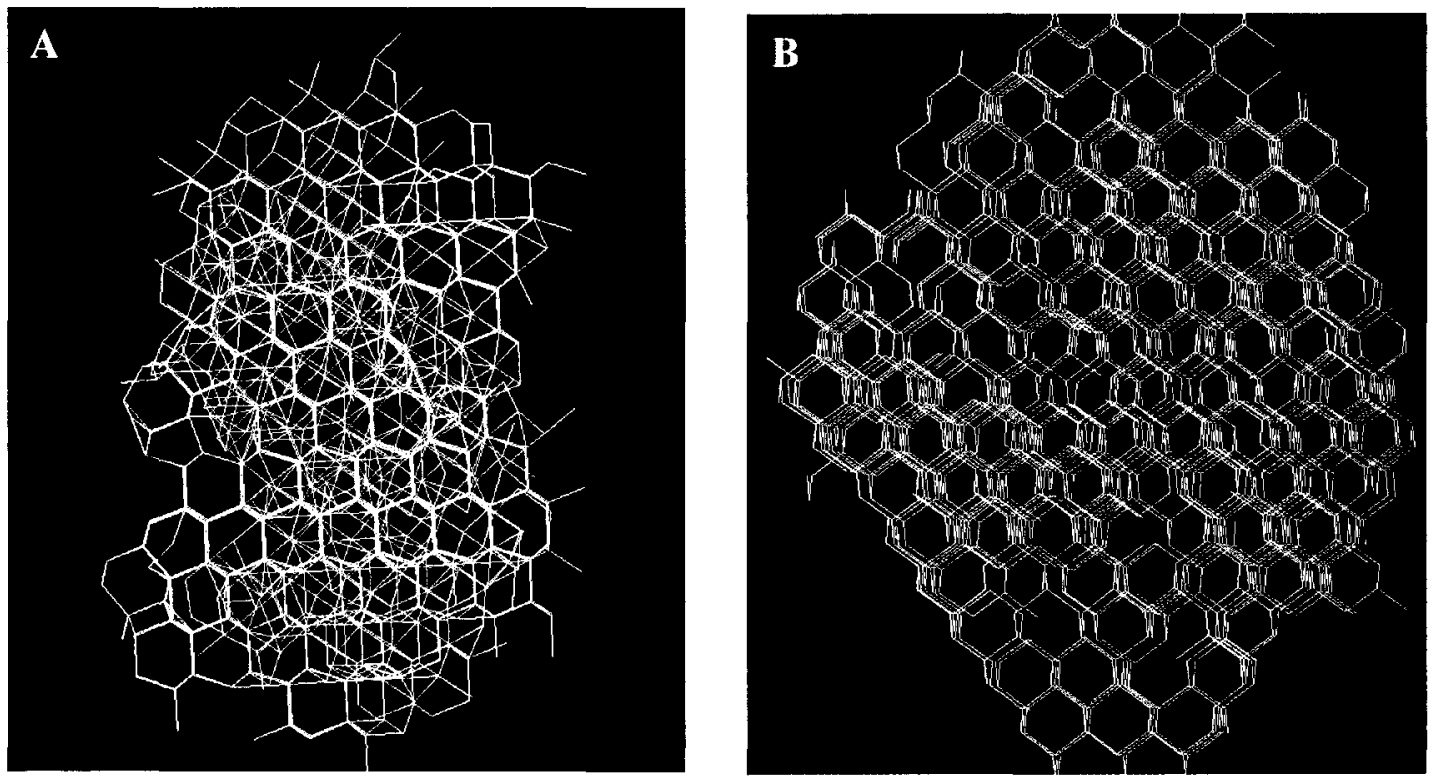

Figure 4.9 Simulated diamond clusters with (a) large amounts of stacking faults and (b) $30 \%$ vacancy in the structure.

\subsection{Intensity ratio calculations from diffraction patterns}

To resolve the mystery of the structure of the fcc carbon and bcc carbon, the relative intensities of the spots in the nanodiffraction patterns were carefully plotted for a 
large number of crystals. These nanocrystals occur in random orientation and also the relative intensities of the spots in the patterns may vary widely with the tilt of the crystal with respect to the incident beam direction. However, observations of relative intensities for recognizable two-dimensional patterns obtained close to a zone-axis orientation should give significant information.

Figure 4.10a shows the relative intensities of the (200) and (111) spots in patterns obtained from [110] orientation of fcc crystals with cell dimensions close to $\mathrm{a}=0.36 \mathrm{~nm}$. The intensities are approximate, estimated visually with the help of a calibration pattern formed by multiple exposures, but were accurate to within about 20 percent. Figure 4.10b shows similar estimates of relative intensities of the (200) and (220) spots for fcc crystals with cell dimensions close to $0.36 \mathrm{~nm}$ in the [100] orientations.
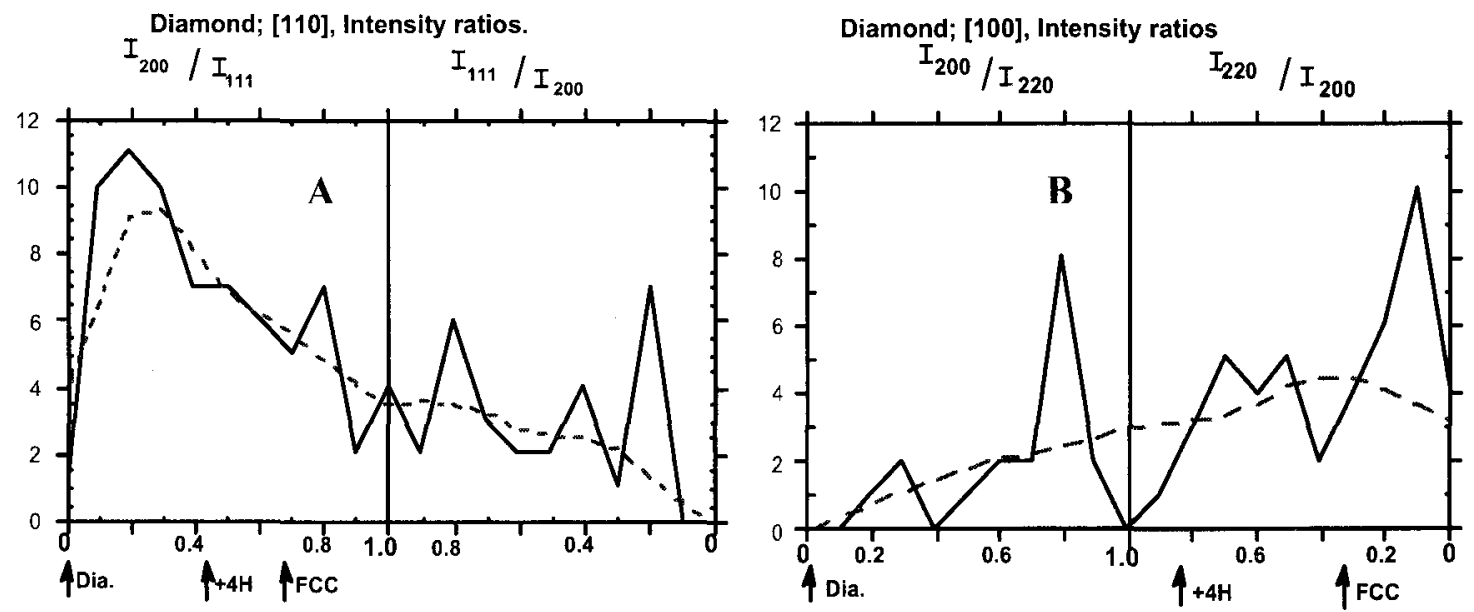

Figure 4.10 Distribution of the observed ratio of (200) and (111) intensities in the nanodiffraction patterns of $n$-diamond crystals $(a=0.36 \mathrm{~nm})$ in the (a) [110] and (b) $[100]$ zone axis. 
For both [110] and [100] orientations, the (200) intensities and the corresponding ratios are zero for normal diamond for diffraction with the usual assumption of kinematical scattering. For the fcc structure in [110] and [100] orientations are indicated in Figure 4.10a-b. The n-diamond structure was observed experimentally to have strong (200) reflections and thus high intensity ratios, for the axial orientations, as indicated in the diagrams. If the n-diamond crystals in the samples had a true fcc structure, one would expect a broad distribution of intensity ratio values around the value expected for fcc. Clearly, the distributions are not centered on the values for the axial orientations of the fcc structure, but appear to be centered about some value intermediate between that for the fcc structure and the zero values expected for normal diamond.

The deviation from the diamond values is emphasized by the observation of many patterns for which the (200) reflection is much stronger than the (111). This could be expected for a fec structure for particular tilts of the crystal, but is very unlikely if the (200) intensity for the axial orientation is very much weaker than the (111).

The thickness of the nanocrystals in our specimens could not be measured directly. Only the lateral extent of the nanocrystals could be seen in dark-field STEM images. It may be assumed that for randomly oriented crystals, the thickness is on the average, approximately equal to the lateral dimensions. For most sample areas, the lateral dimensions of the coherently diffracting regions were seen in the dark-field STEM images to be in the range of 5 to $10 \mathrm{~nm}$. Occasionally, larger crystals, up to $100 \mathrm{~nm}$ in diameter, which appeared to be in the form of thin sheets with numerous structural imperfections, were visible. As well as could be judged, the relative intensities of the diffraction spots from these larger crystals were the same as for the nanocrystals. 
Attempts were made to observe the variation of the relative intensities as the incident beam was translated across a thin edge of a larger crystal, but it was not possible to detect any variation of relative intensities with the assumed increase of thickness. It was therefore concluded that dynamical scattering effects were not an important factor for the interpretation of the relative spot intensities.

\subsection{Structure of the bec phase of carbon}

The peaks for bcc phase appeared consistently at a $=0.31 \mathrm{~nm}$ as shown in Figure $4.3 \mathrm{~b}, 4.4 \mathrm{~b}$ and $4.5 \mathrm{c}$. Also, only bec structures in [110] orientation can give the rectangular patterns such as Figure $4.4 \mathrm{~b}$. Hence, there was a strong indication of the presence of a bcc phase with approximately $\mathrm{a}=0.31 \mathrm{~nm}$.

The relative intensities in these patterns that may be attributed to bec phase did not show any pronounced features that would imply any great deviation from a simple bcc structure. This implied that the patterns came from a bcc carbon structure, with two carbon atoms in a unit cell with $\mathrm{a}=0.31 \mathrm{~nm}$. The $\mathrm{C}$-C distance for the 8 nearest neighbors would then be $0.27 \mathrm{~nm}$.

An explanation of a bcc phase with carbon is possible by imagining two interpenetrating diamond lattices as shown in Figure 4.11. This type of a structure would be quite hard to imagine, considering that diamond has the highest packing density for covalent solids. This proposed structure, if possible, would have a packing density twice the density of diamond $\left(7.08 \mathrm{gm} / \mathrm{cm}^{3}\right)$. The nearest neighbor distance for this structure was calculated to be $0.154 \mathrm{~nm}$, which was the same as that for diamond. Also, each carbon atom would be surrounded by 8 nearest neighbors, but would be bonded to only 4 
of them due to tetrahedral bonding. This structure would give perfect bec diffraction. Incidentally, Proserpio et al. suggested such a structure was suggested for $\mathrm{BeF}_{2}-\mathrm{SiO}_{2}$ and $\mathrm{BeF}_{2}-\mathrm{GeO}_{2}$ compounds [147]. This structure of interpenetrating diamond lattices was called the ice-VII structure. It was suggested that this structure made up of carbon atoms alone would be difficult as each site would be eight-coordinated.

Besides, the lattice parameter calculated for this theoretical structure was 0.178 $\mathrm{nm}$, which is nearly half of that obtained experimentally in this work. Hence, clearly, this structure although may be possible, is not an explanation for the bcc crystals that were observed in this study. 


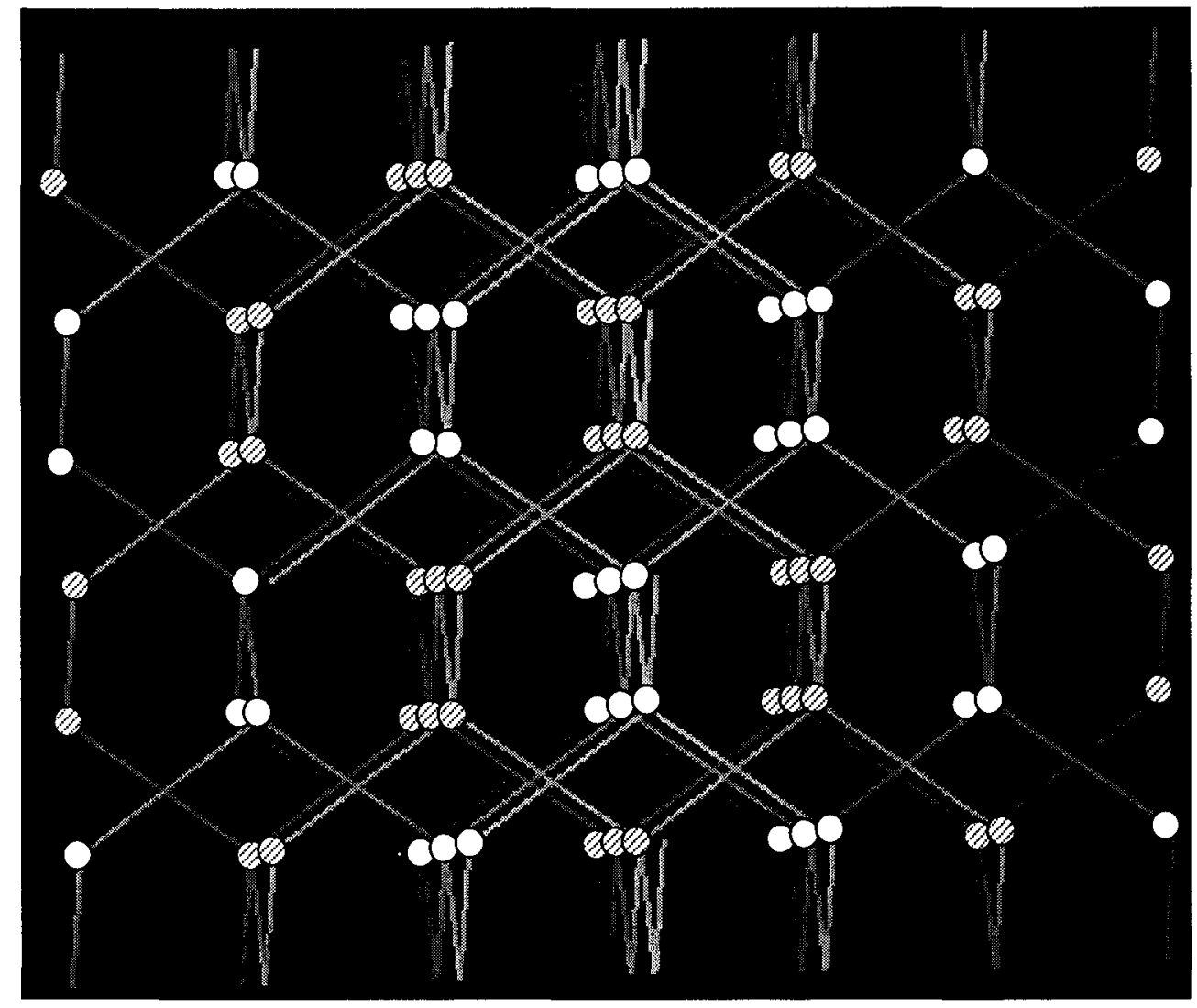

Figure 4.11 Ball and stick model of a proposed bcc phase of carbon with interpenetrating diamond lattices with a-value of $0.178 \mathrm{~nm}$ (white and hashed atoms are the two separate diamond lattices).

\subsection{Possibility of hydrogen incorporation in carbon lattice}

The accepted structure for n-diamond, and also the suggested bcc structure, seems to involve such unprecedented bonding arrangements for carbon atoms that it is inevitable that the possibility of the presence of other atoms in the lattice must be explored. Our attempts to detect a possible contaminant by TEM/EDS were unsuccessful. Also, previous attempts to detect other elements in $\mathrm{n}$-diamond samples by use of EELS 
have not been successful $[24,148]$. Since hydrogen is the key constituent of the gas phase used for synthesis of these crystals, and that it is the only atom, which cannot be readily detected by EELS, the possibility of $\mathrm{H}$ being present in the structure must be considered.

The synthesis of $\mathrm{n}$-diamond, in most cases presented in literature also involved the presence of hydrogen in the gas phase. Theoretical studies of carbon clusters have suggested that hydrogen may have an essential role in bonding at least on the surfaces of the stable clusters [149] and the similar roles for hydrogen were postulated for the explanation of some experimental observations [23, 148]. A simple surface saturation of diamond clusters with hydrogen (clusters suggested by Barnard et. al [150]) did not result in any difference in diffraction patterns from non-hydrogenated diamond clusters. The next possibility was having hydrogen in the lattice of carbon atoms.

The normal diamond structure may be described as consisting of two intertwined fcc structures, with origins at the positions $0,0,0$ and $1 / 4,1 / 4,1 / 4$. If one assumes that the 4 carbon atoms on one of these substructures are replaced by hydrogen atoms, the unit cell content is $\mathrm{C}_{4} \mathrm{H}_{4}$ (or simply $\mathrm{CH}$ ) and the symmetry is fcc, just like the zinc blende structure. The relative intensities of the (200) and (111) reflections, and of the (200) and (220) reflections are as indicated by the arrows labeled " $+4 \mathrm{H} "$ in Figure $4.10 \mathrm{a}$ and $4.10 \mathrm{~b}$. These relative intensity values provide a better account of the distributions of intensity ratios than those for either the normal diamond or pure carbon fcc structures, although some structure a little closer to that of the normal diamond structure (i.e. with a little more scattering power than $4 \mathrm{H}$ on the one sublattice) may give an even better fit.

To provide some further foundation for the suggestion of such a structure, a theoretical analysis was carried out. 


\subsection{Energetic stability of the new carbon phases with hydrogen incorporation}

Calculations were carried out using density functional theory $[151,152]$ with the gradient-corrected local exchange-correlation potential of Perdew and Wang [153, 154] using a VASP code $[155,156]$. Standard periodic boundary conditions were employed with the core electrons represented by ultrasoft pseudopotentials [157] and a plane-wave basis set for the valence electrons. A plane-wave cut-off of $500 \mathrm{eV}$ was found to be sufficient to converge the total energies and geometries. $k$-point sampling during geometry optimization for all structures was carried out on a 11x11x11 Monkhort-Pack grid [158]. Geometry optimization was undertaken using a combination of conjugate gradient (first derivative) and quasi-Newton (approximate second derivative) methods.

The value of the fcc unit cell parameter obtained from the above calculations was $0.3098 \mathrm{~nm}$, which is in good accord with some of the previous theoretical calculation results $(0.3020-0.3082 \mathrm{~nm})[58,59]$. For bcc carbon, the obtained lattice parameter was $\mathrm{a}=0.2389 \mathrm{~nm}$. There is strong evidence indicating that these phases are mechanically unstable at least at atmospheric pressure, however, no evidence exists for the rate of their transformation to relatively stable phases like diamond or graphite $[59,159]$.

The fact that the unit cell parameters have a wide range of values suggest very strongly that these phases have a variable composition, and thus the most plausible candidate is $\mathrm{CH}_{x}$. Accordingly, a number of compositions with either fcc carbon or bcc carbon were examined (Table 4.1). As can be seen from Table 4.1, the unit cell parameter increases smoothly from $\mathrm{C}$ to $\mathrm{CH}_{3}$ (fcc) and from $\mathrm{C}$ to $\mathrm{CH}_{3}$ (bcc) in most cases. The most stable phase is the "bcc" $\mathrm{CH}_{2}$ with $a=0.3107 \mathrm{~nm}$. This phase actually has a positive 
affinity for $\mathrm{H}_{2}$ i.e. for the reaction $\mathrm{CH}_{2} \rightarrow \mathrm{C}+\mathrm{H}_{2}, \Delta E=0.72 \mathrm{eV}$. In contrast to the pure carbon phase, this phase is at a local minimum of energy (metastable).

Table 4.1 Theoretically calculated values for the unit cell parameter, a, and the energy per carbon atom, $\Delta \mathrm{E} / \mathrm{C}$, for various possible $\mathrm{CH}_{\mathrm{x}}$ crystalline phases.

\begin{tabular}{|l|l|l|l|l|}
\hline Composition & Symmetry & Structure & $a(\mathrm{~nm})$ & $\Delta E_{f} / \mathrm{C}(\mathrm{eV})$ \\
\hline $\mathrm{C}$ & $F m-3 m$ & fcc & 0.3098 & 4.61 \\
\hline $\mathrm{CH}$ & $F-43 m$ & $\mathrm{ZnS}$ & 0.3583 & 5.21 \\
\hline $\mathrm{CH}_{2}$ & $F m-3 m$ & $\mathrm{CaF}_{2}$ & 0.3787 & 5.64 \\
\hline $\mathrm{CH}_{3}$ & $F m-3 m$ & $\mathrm{a}$ & 0.3953 & 5.75 \\
\hline & & & & \\
\hline $\mathrm{C}$ & $I m-3 m$ & bcc & 0.2389 & 4.43 \\
\hline $\mathrm{CH}_{2}$ & $P n-3 m$ & $\mathrm{Cu} 2 \mathrm{O}$ & 0.3107 & 3.64 \\
\hline $\mathrm{CH}_{3}$ & $P m-3 n$ & $\mathrm{~A} 15$ & 0.3096 & 7.20 \\
\hline $\mathrm{CH}_{4}$ & $I m-3 m$ & $\mathrm{~b}$ & 0.3326 & 4.22 \\
\hline
\end{tabular}
a. C: $0,0,0 ; \mathrm{H}: 1 / 2,1 / 2,1 / 2$ and $1 / 4,1 / 4,1 / 4$.
b. C: $0,0,0 ; \mathrm{H}: 1 / 4,1 / 4,1 / 4$.

It was determined that for $\mathrm{H}$ on one of the diamond sublattices, such that the unit cell contained $4 \mathrm{C}$ and $4 \mathrm{H}$, the equilibrium unit cell dimension would be $0.358 \mathrm{~nm}$. This in agreement with the experimental observations and distinctly different from the value $0.307 \mathrm{~nm}$ for vacancies in place of the hydrogen atoms (in agreement with results of Pickard et al. [58] and Murrieta et al.[59]). 


\subsection{Discussions}

It was found that a perfect fec structure did not match with the calculated diffraction intensity ratios determined experimentally. Also, the bec phase, that was never reported before for carbon was observed in this work. The possibility of heavier atom contamination, anomalies in diffraction pattern, and other possible defects in crystal structures were eliminated. Hence, the only way these structures could be consistently explained was by the possibility of incorporation of a lighter element. Since hydrogen was used in all the experiments to synthesize n-diamond, it seemed like a possibility that it played a role in these new phases. Thus, it could be possible that hydrogen may be incorporated in the lattice of carbon. Possible structures for fcc and bcc carbon proposed are $\mathrm{CH}$ and $\mathrm{CH}_{2}$ respectively. These structures are energetically stable and also match the intensity ratios discussed before.

An independent confirmation of the presence of $\mathrm{H}$ in the $\mathrm{n}$-diamond structure would be very desirable. While there may be techniques like neutron diffraction and ERDA (Elastic recoil detection analysis), which are capable of detecting hydrogen, they would be useful for a pure phase sample, rather than a sample with mixture of phases. Also, with so many phases present, one would need to know the type of crystal that is being analyzed, before detecting the amount of hydrogen, and only electron nanodiffraction seems to be capable of studying the structure of individual crystals. Any technique for which the volume of the sample examined has dimensions greater than about $10 \mathrm{~nm}$ would not be successful for these samples since the nanocrystals of $\mathrm{n}$ diamond are mostly embedded in amorphous carbon and accompanied by crystals of 
other phases including graphite. The EELS technique has the required spatial resolution but is not sensitive to hydrogen.

An alternative model for the $\mathrm{n}$-diamond structure, not involving $\mathrm{H}$, was proposed by Hirai et al [160]. However, their structures involved peculiar bonding configurations and, for their most favored model, the calculated diffraction intensities were in poor agreement with observations made in these calculations.

The calculations have shown that the proposed $\mathrm{CH}_{2}$ bcc is metastable with $\mathrm{a}=$ $0.31 \mathrm{~nm}$, as observed. The relative intensities of the bcc diffraction spots would however be modified by the inclusion of the $\mathrm{H}$ atoms but the differences would be difficult to confirm for the nanocrystals in random orientation. Some (111) reflections would appear weakly. Such reflections, too weak for reproduction, have been observed in a few of the bcc [110] patterns.

For the proposed bcc structure the insertion of four hydrogen atoms would give a tetrahedral coordination for the $\mathrm{C}$ atoms with a $\mathrm{C}-\mathrm{H}$ distance of about $0.14 \mathrm{~nm}$, as against $0.16 \mathrm{~nm}$ for the fcc structure (See Figure 4.12).

In general, the type of bonding between $\mathrm{C}$ and $\mathrm{H}$ in both these structures would be difficult to explain. As such, carbon phase in the fcc and bcc lattice would seem to involve unprecedented bonding, else they would exist simply as diamond or graphite. Hence, as a pure speculation, one can imagine the bonding in $\mathrm{CH}_{\mathrm{x}}$ to be similar to metal hydrides, or boranes [161], where hydrogen atoms can be shared by more than one atom. The electrons in this structure may be highly delocalized, hence satisfying the electrons required by $\mathrm{C}$. 


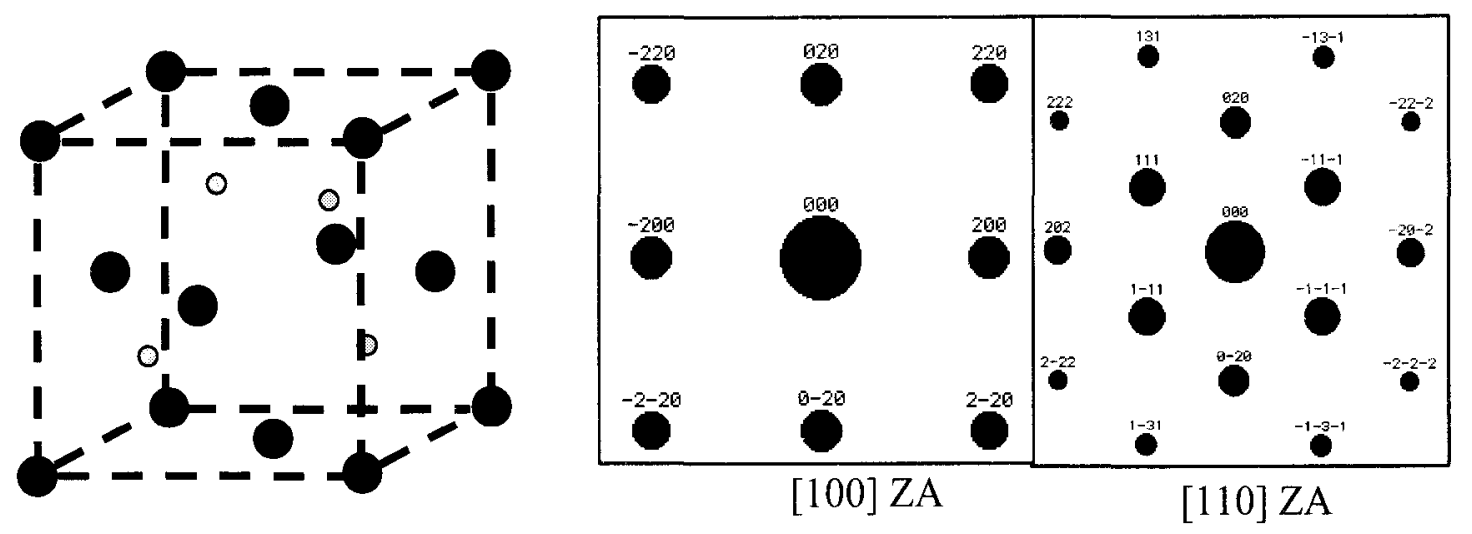

A
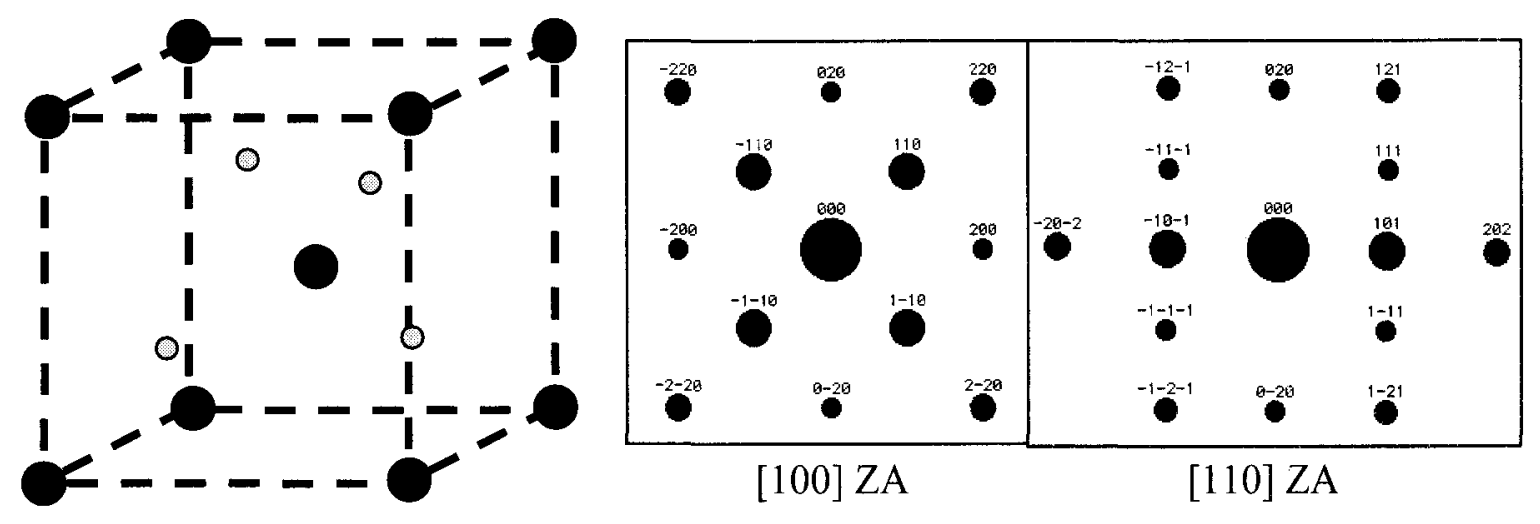

B

Figure 4.12 Proposed structures of (a) $\mathrm{CH}$ fcc crystal with a lattice parameter of $0.36 \mathrm{~nm}$ and (b) $\mathrm{CH}_{2}$ bcc structure with a lattice parameter of $0.31 \mathrm{~nm}$ and their corresponding diffraction patterns in the $[100]$ and [110] zone axis.

There was no direct evidence for a link to diamond nucleation in this study, except for the fact that a few crystals in this nanocrystalline mixed phase samples were perfect diamond cubic. The fcc and bcc crystals are also cubic phases of carbon. Two interpenetrating fcc crystals gives rise to a diamond cubic structure and two interpenetrating diamond cubic crystals give rise to a bcc structure. This may in a way suggest a possible link of these nanocrystalline carbon phases with diamond. The bias 
enhanced diamond nucleation discussed in reference [55] determined intermediate forms of amorphous clusters which contain almost 20 to $30 \%$ of atomic hydrogen. Eventually these clusters precipitated some $\mathrm{sp}^{3}$ bonded atoms. However, the crystal structure of these diamond nanocrystals was studied using selected area diffraction, which would probably not be as informative as a single crystal nanodiffraction patterns discussed here. In another work [52], diamond nucleation was explained from the hydrogenation of graphite edges. Both of the above explanations for diamond nucleation involved the role of hydrogen in the formation of diamond from non-diamond intermediate phases. Thus crystalline phases involving $\mathrm{CH}_{\mathrm{x}}$ found at the nanoscale in this study could possibly act as intermediate phases prior to diamond nuclei formation, and hence may explain the pathway for diamond nucleation. This hypothesis extends the existing theories for diamond nucleation $[52,55]$.

Figure 4.13 indicates a three-dimensional thermodynamic plot ${ }^{\dagger}$ of the free energy of hydrocarbon species in the gas and solid phase. There are two paths indicated in the plot for gas phase condensation to diamond. Path A leads to the formation of graphite, which needs to cross a high activation energy barrier before it converts to diamond, while Path B is the preferred path, which does not involve any such activation barrier. Instead, from Path $\mathrm{B}$, a minimum energy point is reach at $\mathrm{H} / \mathrm{C}$ of nearly 1 , which further gets converted to diamond or can move to higher $\mathrm{H} / \mathrm{C}$ ratios. $\mathrm{H} / \mathrm{C}$ of the proposed $\mathrm{FCC} \mathrm{CH}$ phase is 1, while that of the proposed $\mathrm{BCC} \mathrm{CH}_{2}$ phase is 2. Thus, this plot provides further indication that the proposed $\mathrm{CH}_{x}$ phases could be the intermediate phases to diamond nucleation. Obviously, the free energy of these new $\mathrm{CH}_{x}$ phases needs to be reevaluated to obtain the exact position of these new phases in Figure 4.13.

† J. C. Angus, Case Western University, unpublished data (1994). 


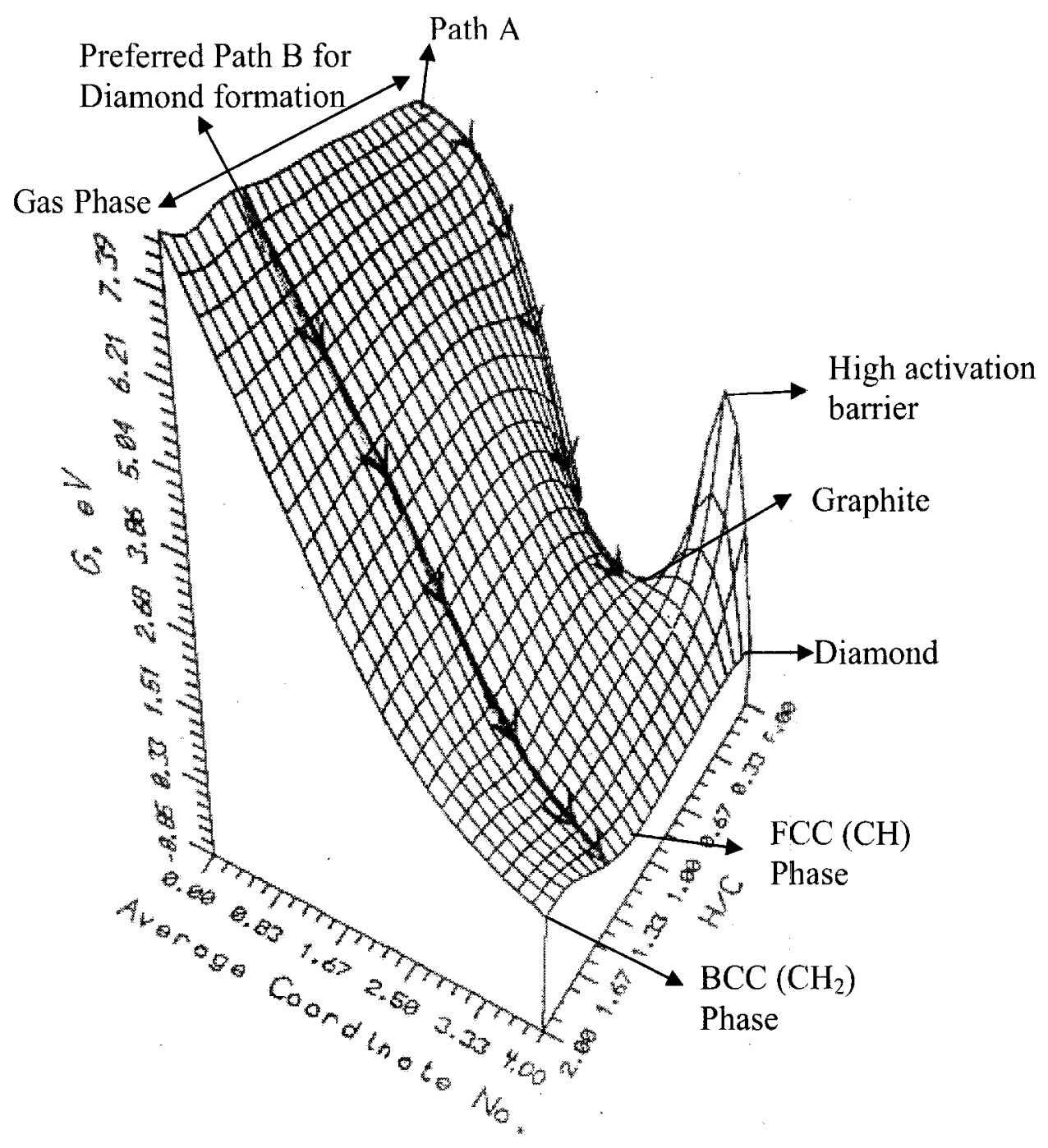

Figure 4.13 Three-dimensional thermodynamic plot of the free-energy of hydrocarbon species in the gas and solid phases as a function of co-ordination numbers and $\mathrm{H} / \mathrm{C}$ ratios.

The proposed structures for the new phases involve a very high density of hydrogen atoms suggesting their possible interest as hydrogen-storage media. These crystalline metastable $\mathrm{CH}_{x}$ phases could explain the intermediate structures that might be present during diamond nucleation. 


\subsection{Summary}

Nucleation experiments for carbon crystallization from vapor phase were conducted by condensing carbon onto platinum wires immersed directly into the dense part of a microwave plasma. Nanodiffraction technique was used extensively to study the electron diffraction from each nanocrystal. The results indicated that most of the cubiccarbon phases had structures of fcc-carbon and bcc-carbon. Theoretical calculations of diffraction patterns from simulated clusters were used to explain the structure of these new phases. The possibilities of heavy element contamination, structural defects such as stacking faults, vacancies, and mixed $\mathrm{sp}^{2} / \mathrm{sp}^{3}$ phases were eliminated using diffraction simulations. The evidence for the n-diamond with a simple fcc structure with just $4 \mathrm{C}$ in a unit cell was shown experimentally (using intensity ratio in spot-patterns) to be quite unlikely. This work suggests the incorporation of $\mathrm{H}$ in the lattice of carbon atoms, which is supported experimentally and theoretically. The seemingly improbable structure with $\mathrm{H}$ incorporation is yet less improbable than the structure without $\mathrm{H}$. The proposed existence of a bcc structure with the addition of hydrogen and $\mathrm{a}=0.31 \mathrm{~nm}$, may also seem improbable but is even more strongly supported theoretically. 


\section{CHAPTER 5}

\section{KINETIC FACETING OF MULTIPLY TWINNED DIAMOND CRYSTALS DURING VAPOR PHASE SYNTHESIS}

\subsection{Introduction}

Diamond crystal growth from the vapor phase often results in multiply-twinned crystal shapes: perfect decahedron, star-decahedron, do-decahedrons, star-dodecahedrons, perfect icosahedrons, dimpled-icosahedrons [94, 96, 98], along with untwinned crystals: octahedrons and cubo-octahedrons. Different forms of the basic five-fold and twentyfold crystals (shown previously in Figure 2.7) are shown in Figure 5.1 (for the reader's convenience). Decahedral and star decahedral morphologies are both bounded primarily by $\{111\}$ facets. Hence, the $\alpha$-factor (modified Wulff's theory for minimization of surface energies, described in Chapter 2, section 2.5) must intuitively be the same for both the crystals, making it difficult to predict the occurrence of one morphology versus the other. It is thus necessary to understand the kinetic mechanisms that differentiate these two five-fold morphologies, to predict their occurrences.

The mechanism for the formation of basic five-fold (decahedron) and twenty-fold (icosahedron) morphologies was explained with occurrence of two and three stacking errors during growth of different $\{111\}$ surfaces, respectively [97,162]. This hypothesis further suggests that a decahedral crystal with one additional stacking error during growth would transform into an icosahedral crystal. However, no experimental evidence was 
provided for this hypothesis. By performing sequential growth experiments, one could track the changes in morphology of a crystal with growth, and there would be a good probability of observing this transition of a five-fold to a twenty-fold multiply twinned crystal.
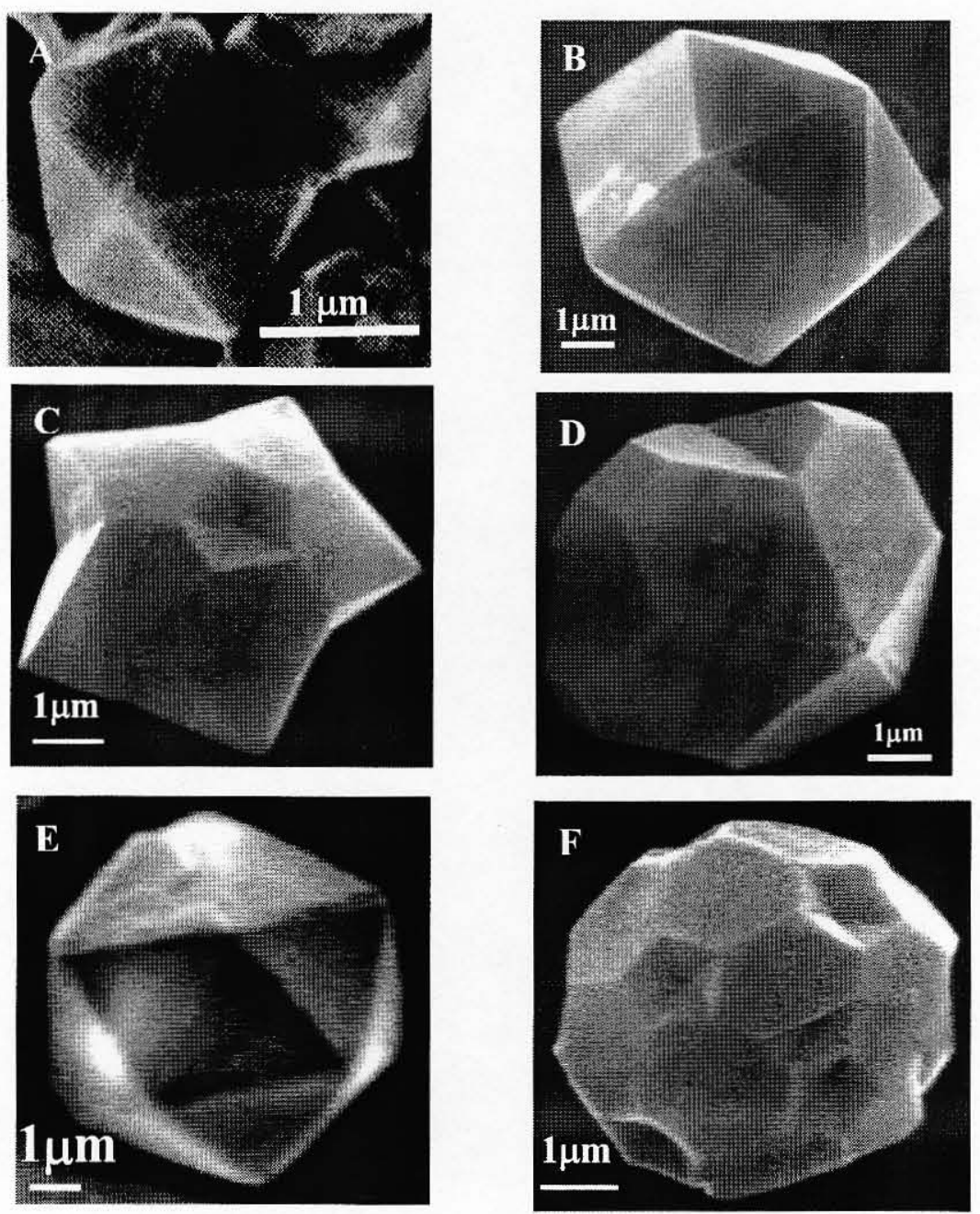

Figure 5.1 SEM images of different multiply twinned diamond crystal morphologies (a) decahedron (courtesy ref. 98) (b) do-decahedron (c) star-decahedron (d) stardodecahedron (e) icosahedron (f) dimpled icosahedron 
The growth of a diamond crystal occurs with the reaction of methyl or other radical species with the existing diamond nuclei that are already formed on the surface. The gas phase radicals chemisorb onto the surface in two ways, the staggered conformation (Figure 2.6a Chapter 2) and the eclipsed conformation (Figure 2.6b, Ch 2) with respect to the surface atom. Eclipsed conformation leads to a stacking error on the $\{111\}$ surface. The detailed adsorption and desorption kinetics of carbonaceous species with the growing crystal surface, number and position of stacking faults govern the overall morphology of diamond. Since every adsorption and desorption event cannot be tracked, simulations of crystal growth is treated with stochastic kinetics.

Kinetic Monte Carlo (KMC) is an efficient stochastic technique to simulate the evolution of crystals to physical sizes of at least several nm. The KMC simulations for studying the evolution of single crystals of diamond in three dimensions involving the adsorption and desorption of methyl radicals was first described by Sunkara and Angus $[96,97,162]$. Based on this methodology, for an untwinned precursor molecule, a number of morphologies were explained such as octahedron, cubo-octahedron while for cyclically twinned precursors the final morphologies were decahedrons and icosahedrons [97]. However, this treatment was not conducive for exploration of an expanded surface chemistry set and treating different types of surface sites. The reason for kinetic faceting like appearance of dimples, groves, additional $\{111\}$ facets that make a star-shape could not be explained using these simulations. Thus, in this study, a more detailed sitespecific chemistry with a more efficient $\mathrm{KMC}$ technique is used to explain the kinetic preference of a star-decahedral crystal to a decahedral crystal and the reasons for obtaining dimples in multiply-twinned crystals. 
This chapter begins with the results from the interrupted growth experiments to study early stages of growth of multiply-twinned diamond crystals. Theoretical studies carried out using KMC simulations are discussed next, with a detailed explanation on the simulation technique, and site-specific chemistries used. The comparisons are made between experimentally grown crystals and simulated crystals to determine the possible molecular level processes responsible for the observed morphologies and the chemistry involved in the diamond crystal growth.

\subsection{Experimental studies on the morphology evolution of twinned diamond crystals}

Interrupted growth experiments were performed to observe the change in morphology of individual crystals upon growth from the vapor phase. Atomic force microscopy (AFM) studies were used to determine angles between the various facets of the crystals.

\subsubsection{Results from interrupted growth experiments}

Diamond crystals were grown on unseeded $\mathrm{Si}$ substrates under lean growth conditions $\left(1-2 \% \mathrm{CH}_{4} / \mathrm{H}_{2}\right)$. After the growth for about 2-3 hours, the crystals shapes on the substrate were observed and mapped using an SEM. Generally, several crystals with an intermediate shape (between star-shape and icosahedron) were tracked. The substrates were loaded back into the reactor and re-grown for about 1 hour. This sequence was repeated 3-4 times.

Most of the times the crystals when re-grown developed significant extraneous faceting. Twins were developed on the $\{111\}$ faces. Hence, it was impossible to observe 
the change in the overall morphology of these crystals. Also, if the surface of the facets of the crystal were not clean, or did not have the same surface termination as existed during the previous shut-down, the development of more defects on the surface occurred. Hence, the number of significant changes in morphology observed was small due to the practical difficulties encountered during these experiments. Some important observations made from these experiments and their inferences are listed below:

(1) Almost all of the five-fold twinned crystals grown in the microwave plasma were star-shaped decahedral crystals. These crystals had a characteristic dimple at the center, with additional $\{111\}$ facets along the twin planes (these are shown clearly in Figure 5.2). .
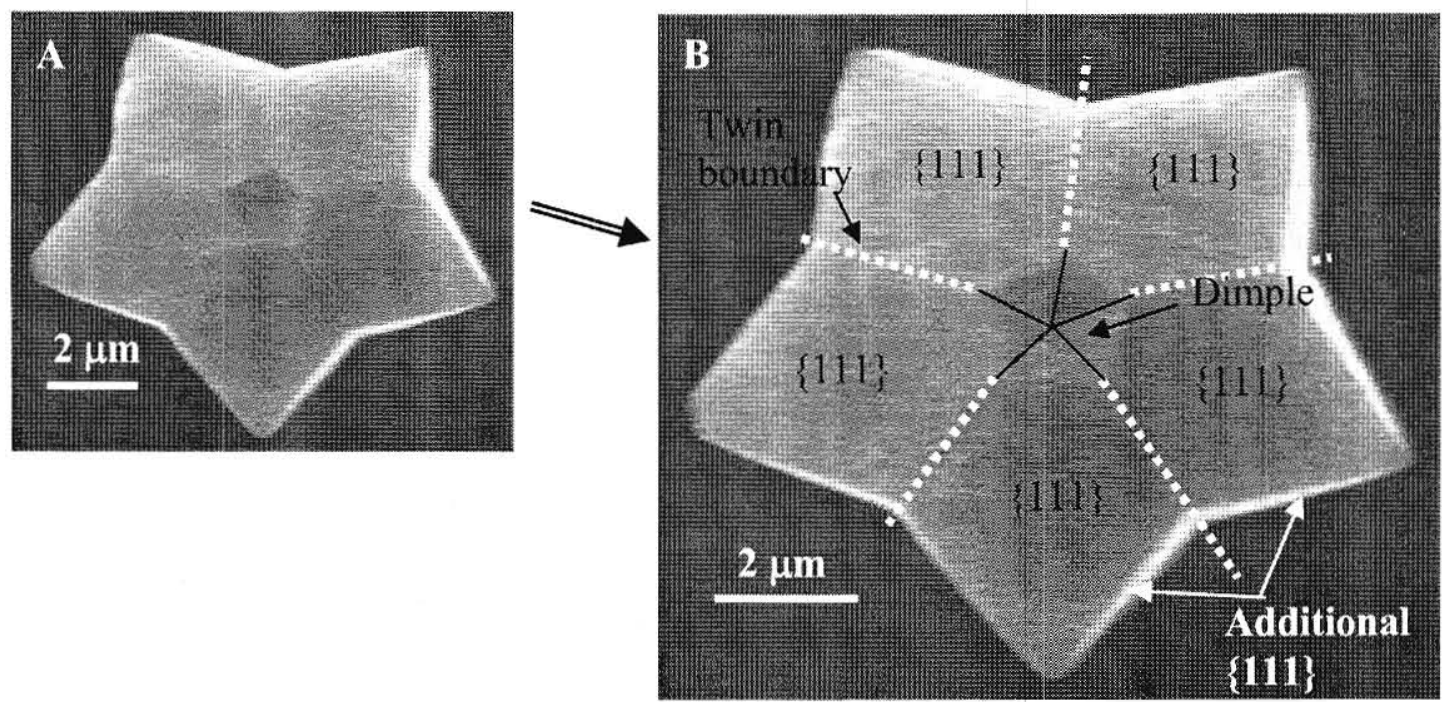

Figure 5.2 (a) SEM image of experimentally grown star-shaped diamond crystal. (b) Indexing different planes and features of the star-shaped crystal (Curly brackets indicate planes, square brackets indicate directions). 
A sequence showing a star-shaped crystal re-grown from $4 \mu \mathrm{m}$ to $12.5 \mu \mathrm{m}$ under the same growth conditions $\left(2 \% \mathrm{CH}_{4} / \mathrm{H}_{2}\right)$ is shown in Figure 5.3. Under the same growth conditions, the overall shape of the crystal did not change, but for the increase in size. After the crystal size reaches about $6 \mu \mathrm{m}$, the facets begin to roughen, and subsequently they develop defects and microfacets.

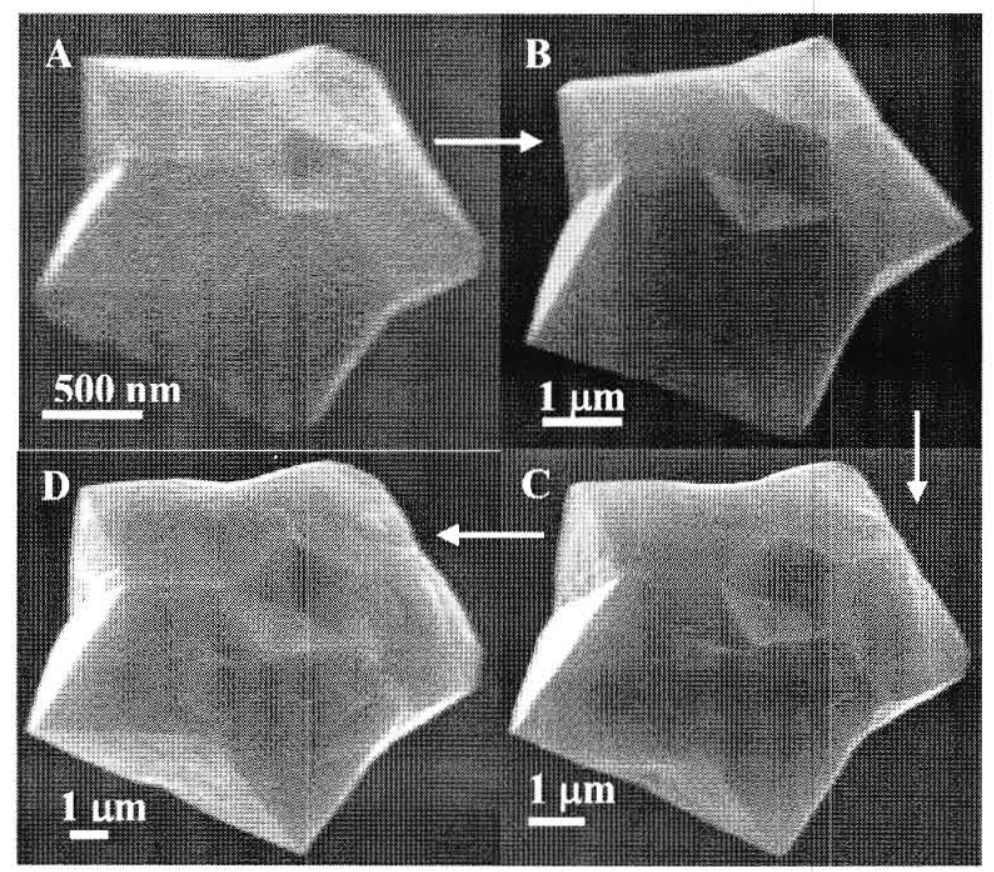

Figure 5.3 SEM images of the sequential growth of a star crystal from (a) $4 \mu \mathrm{m}$ after 3 hours, (b) $6 \mu \mathrm{m}$ after additional one hour, (c) $8.3 \mu \mathrm{m}$ after additional one hour, and (d) $12.5 \mu \mathrm{m}$ after additional 1.5 hours of growth

(2) A star-shaped crystal generally had a dimple, but in some rare cases, these dimples would fill up and then open-up again during growth. The size of the dimple at the center of the star changed with growth at the same conditions. This could occur possibly due to the bunching of steps at the five-fold twin center. A point to be noted 
here is that there were no $\{100\}$ facets with the occurrence of this phenomenon. See Figure 5.4.

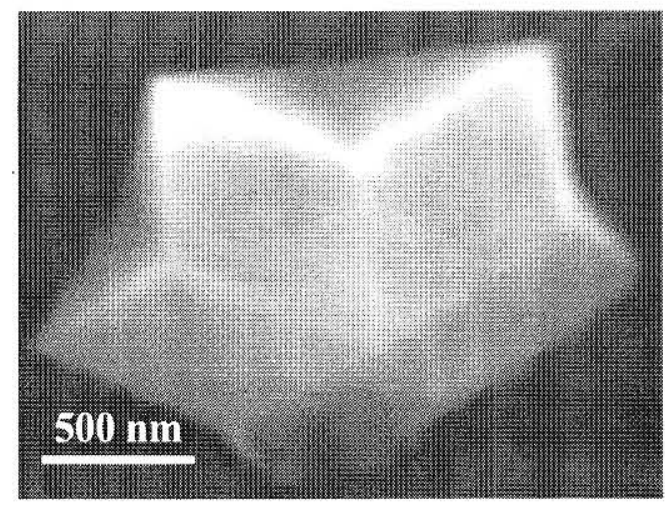

(a)

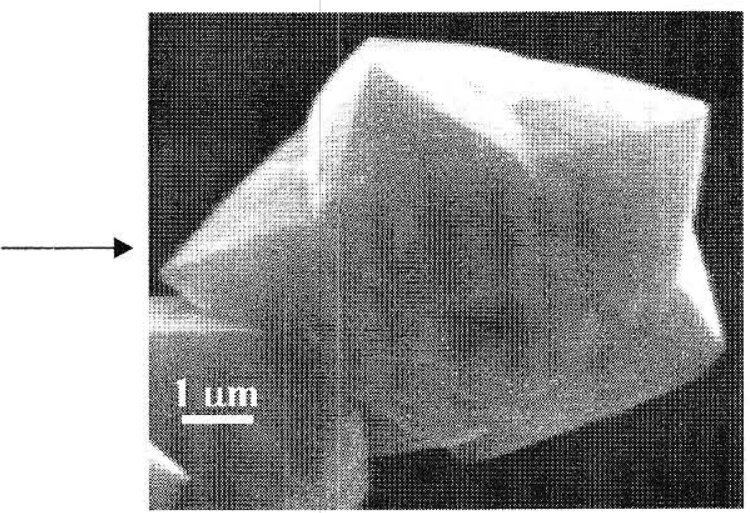

(b)

Figure 5.4 SEM image of (a) star-shaped diamond crystal with no dimple and (b) (a) regrown for one hour at same conditions with the dimple re-appearing.

(3) In a few cases, star-dodecahedral crystals (star-shaped crystals with additional $\{100\}$ facets) converted to star-shaped crystals with re-growth at lower temperatures and higher methane concentrations, making the $\{100\}$ facets disappear. See Figure 5.5a-b. The reverse case also occurred, in which the star-shaped crystals converted to dodecahedral crystals on re-growth, losing their dimples at the center, filling up the additional $\{111\}$ facets and developing $\{100\}$ facets (Figure 5.5c-d). The evolution of a star-decahedral shape to a star-dodecahedral shape (or vice versa) for crystals seemed to follow a similar relationship as that of octahedral to cubo-octahedral. 

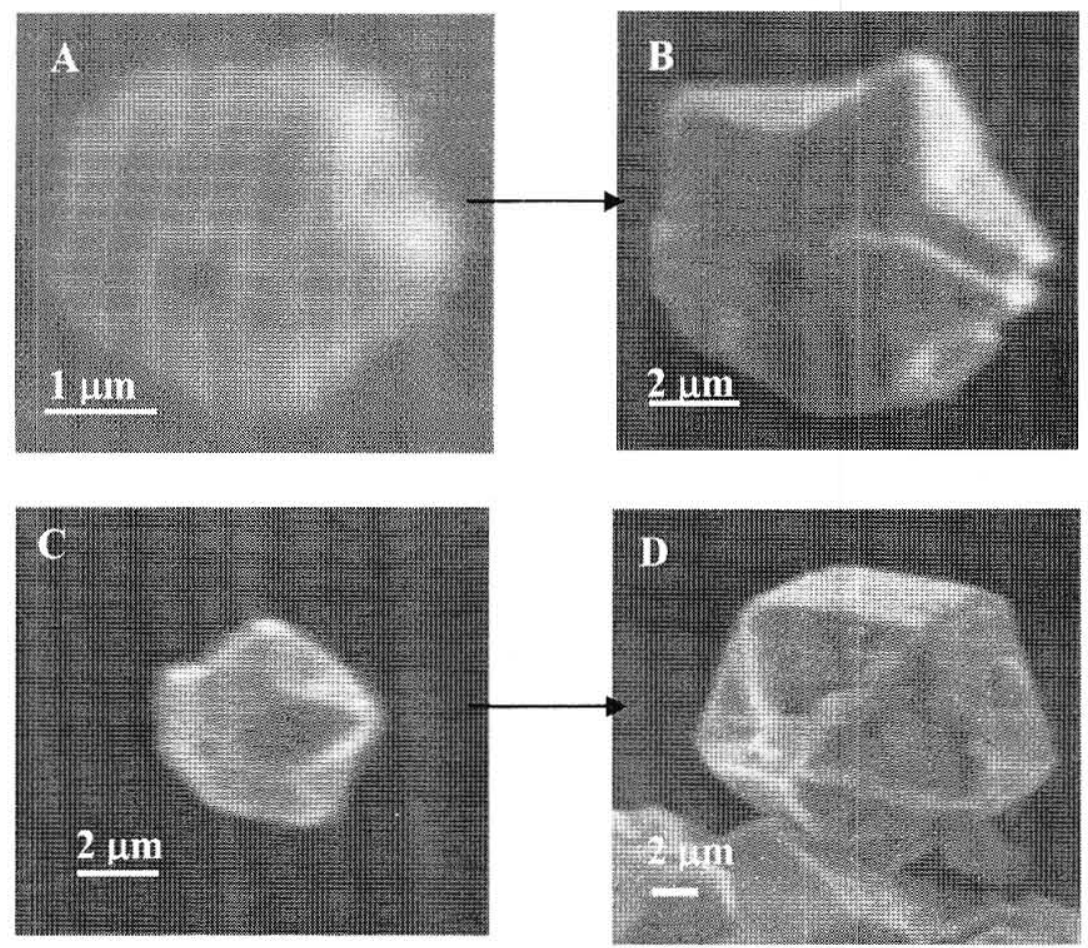

Figure 5.5 SEM images of (a-b) change in morphology of star-dodecahedral to a stardecahedral with decrease in temperature upon re-growth and (c-d) change in the morphology of a star-decahedral to a dodecahedral with increase in temperature upon regrowth.

(4) A number of experiments were performed to track the shape evolution of individual crystals at different stages of growth. In most cases, the five-fold twinned crystals maintained their shape during re-growth experiments. However, the re-growth of an intermediate form $(<1 \mu \mathrm{m})$ shown in Figure 5.6(a), containing only one five-fold dimple, into a nearly complete icosahedron (Figure 5.6(b)) by developing additional fivefold dimples, could be captured. This evolution could be attributed to an additional stacking error during growth. In another case, as shown in Figure 5.6(c), an additional stacking fault seemed to have occurred on a completed star-decahedron, forming a 
second dimpled five-fold center. However, numerous sets of experiments tracking the evolution of a star-shaped crystal failed to provide direct evidence of this evolution, i.e., a crystal starting with a perfect decahedral or star-decahedral shape converting into icosahedral shape upon re-growth at different or similar conditions. To observe such a change, one would have to track extremely small (few nanometers) sized crystals and regrow them to tens of micrometers avoiding any overgrowth, hillocks, or any damage to the surface.
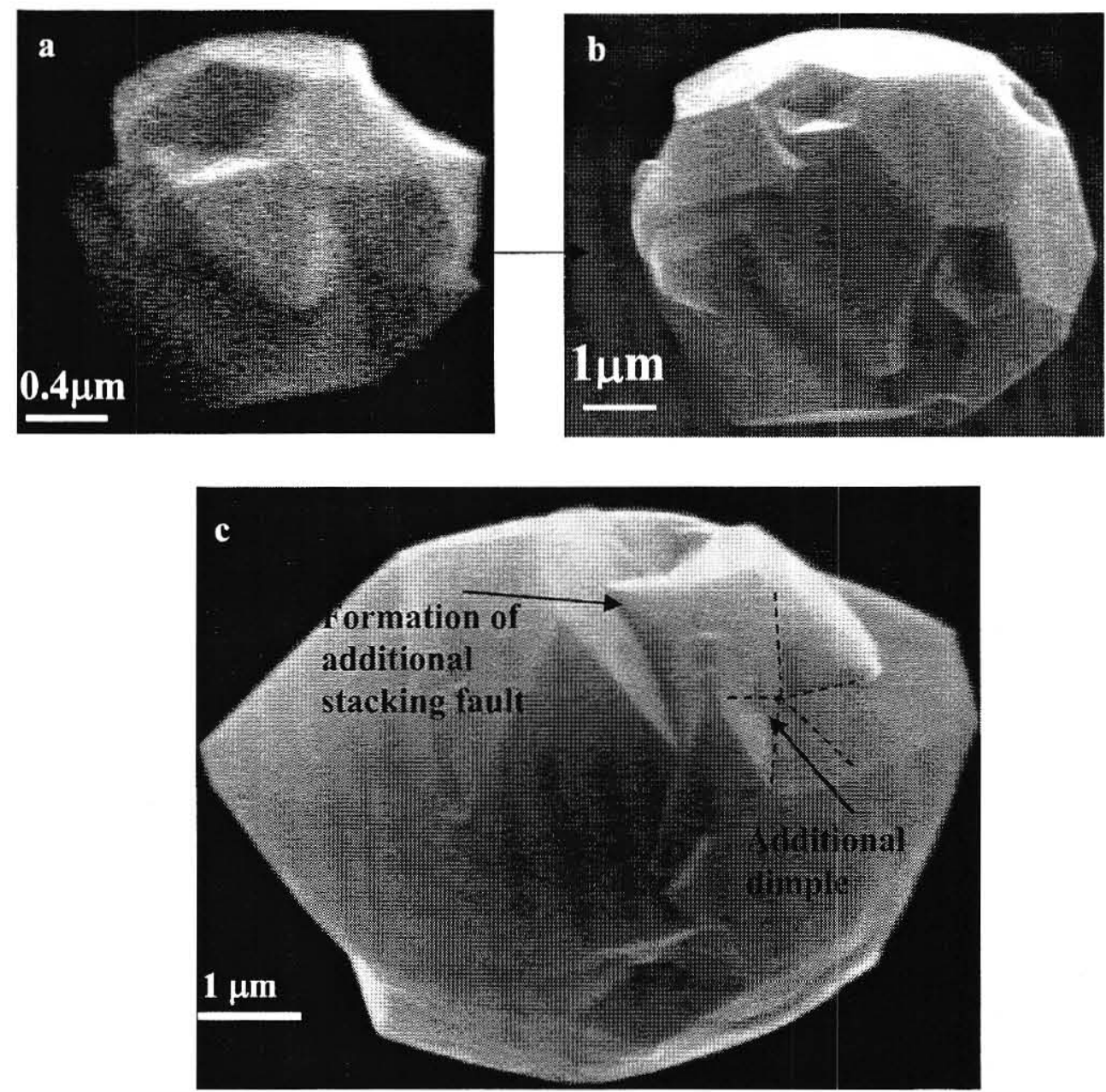

Figure 5.6 SEM image of (a-b) an intermediate shape crystal re-grown to a completed icosahedron and (c) formation of an additional stacking fault in a star-decahedral crystal, giving rise to an additional dimple 


\subsubsection{AFM studies on star-decahedral diamond crystals}

Experimentally, it was observed that the size of the dimple increases with the growth of the star-decahedron at conditions and decreases in size when the temperature during growth increases significantly, with or without the development of $\{100\}$ facets. On the other hand, dimples could be non-existent with the absence of any $\{100\}$ facets in a star-decahedron (Figure 5.4a). In order to understand the faceting inside the dimple, AFM was carried out to determine the planes that are inside the dimple.

Figure 5.7 shows the AFM image taken on a dimple of a star-crystal seeming to lie relatively flat on a substrate (as evidenced by the SEM image). Out of the 4 successful AFM scans on the dimples on four different star-decahedral crystals, three indicated the angle between the dimple plane and the outer $\{111\}$ plane $\left(\theta_{1}\right)$ to be $\sim 109^{\circ}$ while one indicated the angle to be $\sim 140^{\circ}$. Corresponding to these angles, the facets inside the dimple could be either $\{111\}$ (in agreement with prior work [163]) or $\{115\}$. In both these cases (whether $\{111\}$ or $\{115\}$ ), the angle between the facets of the dimple $\left(\theta_{2}\right)$ would be $109^{\circ}$. The observation of high index faceting inside dimples was a further indication of kinetic faceting resulting with enhanced etching/growth of certain sites to slow down the step propagation and allowing bunching of steps. 


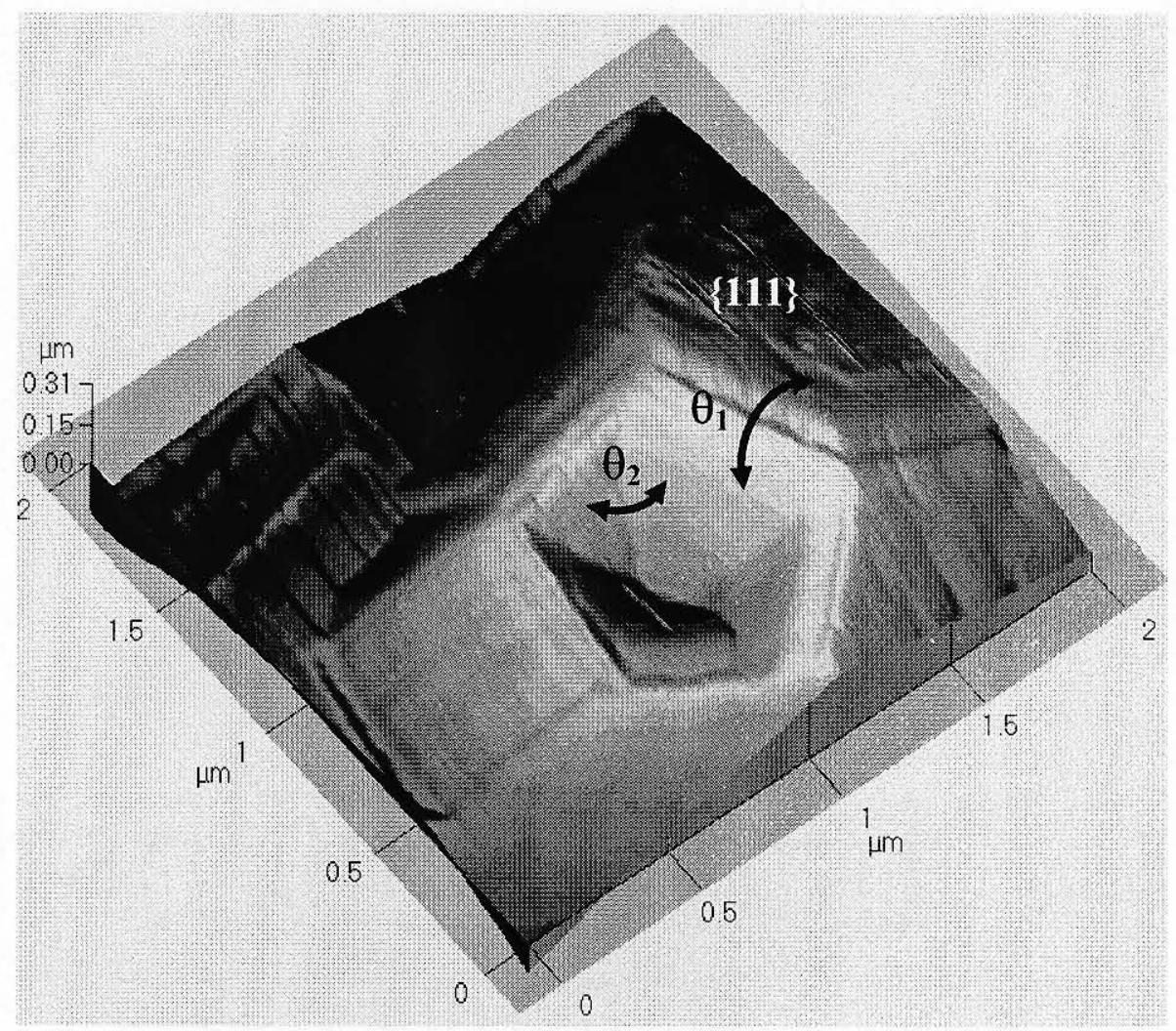

Figure 5.7 AFM scan on a star-shaped diamond crystal. $\left(\theta_{1}\right.$ and $\theta_{2}$ are the two angles measured, which have their meaning as indicated in the figure).

The main conclusions drawn from the experiments on early stages of growth of diamond were that most of the features of multiply twinned crystals such as additional $\{111\}$ facets, dimples, and $\{100\}$ facets were a result of kinetic faceting. Stacking faults mainly govern the overall shape, whether the crystal is untwinned, five-fold twinned and twenty-fold twinned. Features of star-decahedral dimples or additional $\{111\}$ facets are governed by kinetics events that occur during growth. However, the exact events that control this faceting are not known. 


\subsection{Simulations for kinetic faceting of multiply twinned diamond crystals}

Kinetic Monte Carlo (KMC) simulations are stochastic simulations, based on the algorithm of choosing an event (a reaction, in this case) at random out of a set of events at a particular time interval. Sunkara and Angus [97] were the first to use a threedimensional $\mathrm{KMC}$ simulation to explain faceting the evolution of untwinned (octahedrons, cubo-octahedrons) and multiply twinned (decahedrons, dodecahedrons, icosahedrons) single-crystals of diamond. A similar treatment is made here, but with a modified $\mathrm{KMC}$ technique, which makes it easier to incorporate a more detailed sitespecific surface chemistry.

\subsubsection{Simulation methodology}

The KMC technique, as the name suggests, is a stochastic technique for dealing with a large number of surface reactions. The simulation begins with a seed crystal (cluster of a few atoms). Detailed surface-reaction chemistry can be considered, with known or pre-defined rate constants. Reactions (adsorption or desorption events) are picked randomly, based on their respective probabilities. Thus, crystal growth is simulated.

\subsubsection{Assumptions on surface chemistry}

Certain assumptions/simplifications on surface chemistry were made in the KMC simulations used this work:

1. The rate of adsorption at any surface site, $S$, depends on its neighborhood, i.e. the first, second neighbor interactions (explained in detail further). 
2. Hydrogen adsorption and desorption events are neglected as they are much faster than methyl radical adsorption desorption events.

3. The gas phase species considered is (methyl radical) $\mathrm{CH}_{3}$. Other gas phase species can be included, for instance $\mathrm{C}_{2} \mathrm{H}_{2}$, but due to the neglect of hydrogen recombination reactions, the effect of this species would just be two consecutive carbon atom additions on the surface.

4. Growth mainly occurs by the following reaction

$\mathrm{CH}_{3} \cdot \mathrm{S} \cdot \rightarrow \mathrm{S}-\mathrm{CH}_{3}$

While etching during growth occurs by the following reaction:

$\mathrm{S}-\mathrm{CH}_{3} \rightarrow \mathrm{S} \cdot+\mathrm{CH}_{3}$.

$\mathrm{S}-\mathrm{CH}_{2}-\mathrm{S} \rightarrow 2 \mathrm{~S} \cdot+\mathrm{CH}_{2}$.

Thus, growth can occur on atoms bonded 2 or 3 times to the surface, while etching can occur on atoms bonded 1 or 2 times to the surface. Preferably, when an atom is bonded just once to the surface, without having any neighbor interactions, adsorption is not carried out to this atom, as this event would lead to the formation of polymeric chains not allowed for covalent bonding of diamond. In addition due to excess hydrogen in diamond growth environment, formation of polymeric chains are avoided [50].

5. Surface diffusion is neglected (a valid assumption for covalent solids such as diamond).

6. There is no reconstruction of the growth surfaces (valid for temperatures up to $1300 \mathrm{~K})[72]$. 
7. Stacking faults are not allowed to occur during the simulations. The number of stacking faults that can occur are incorporated into the seed itself. The reason being, the purpose of the simulations was to see the effect of surface chemistry on the kinetic faceting such as additional $\{111\}$ facets of star-shaped decahedral crystals and dimples.

\subsubsection{Type of surface sites}

Since KMC simulations are based on site selective chemistry, the surface is divided into the following different types of sites. These sites are vacant bonding positions, where an atom can adsorb. Diamond, with $\mathrm{sp}^{3}$ bonding has 4 available bonds. The available sites for bonding are always on surface atoms, which have at least one site available for bonding. Each plane of diamond is characterized by different available bonds on the surface sites. The reader is referred to Appendix 1 for the different types of primary planes and their respective characteristic surface bonding in diamond.

The following is the list of surface sites that are specified in the simulations. An important point to be noted is that these are vacant sites available at the surface atoms, and they do not refer to the surface atoms themselves.

Type 1: These sites are typically found on the $\{111\}$ surface, in which a $C$ atom is bound only once to the surface. These singly bonded carbon atoms thus have 3 available sites for bonding. Because they are bound only once to the surface, the atoms containing these sites are capable of rotation (eclipsed or staggered) in the absence of any neighbor interactions. Adsorption at such sites gives rise to polymeric chains and must be avoided 
for diamond $\mathrm{sp}^{3}$ bonding. Thus, they always desorbed from the surface. Figure 5.8 illustrates this surface site. However, these sites they tend to occupy a large fraction of surface sites.

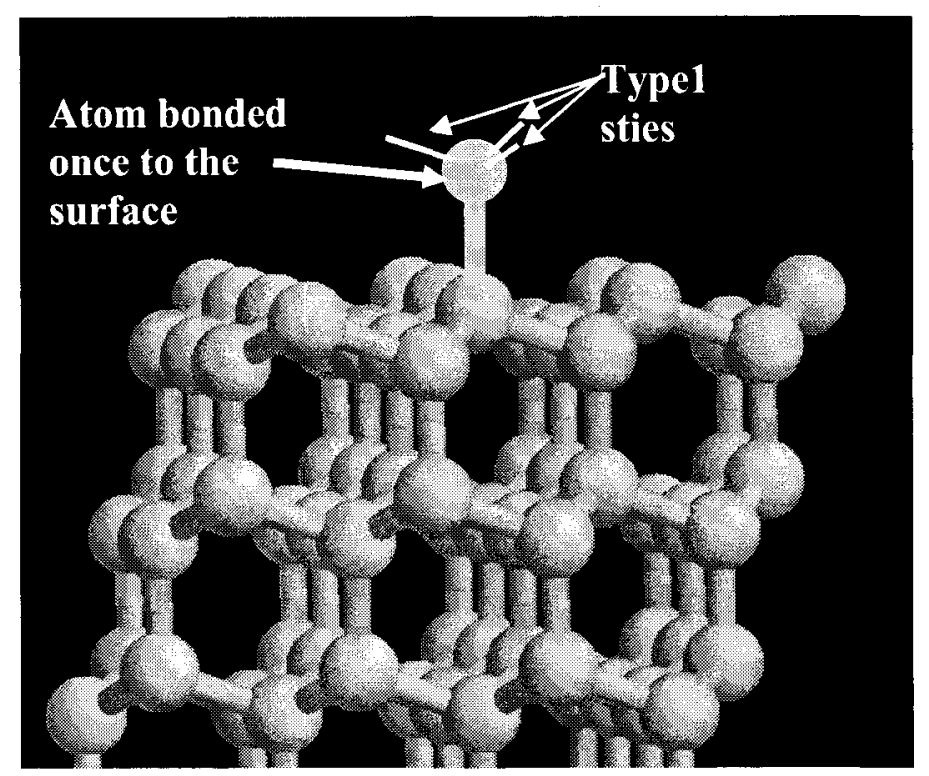

Figure 5.8 Ball and stick model of Type- 1 site, typically found on diamond $\{111\}$ surface.

Type 2: This is an available site on an atom bonded twice the surface, with a second neighbor available. The second neighbor available can be bonded once, twice or thrice. This is specifically called a "kink" site in diamond $\{111\}$. See Figure 5.9. Included in this category is also site on atoms bonded thrice to the surface, with $2^{\text {nd }}$ neighbors, which are bonded at least twice. Broadly speaking, these sites are found on $\{100\}$ surface. These sites are primarily responsible for formation of a 4 atom cluster (stable step) on $\{111\}$ surface and for step-propagation. Adsorption as well as desorption may occur at these type of sites. 


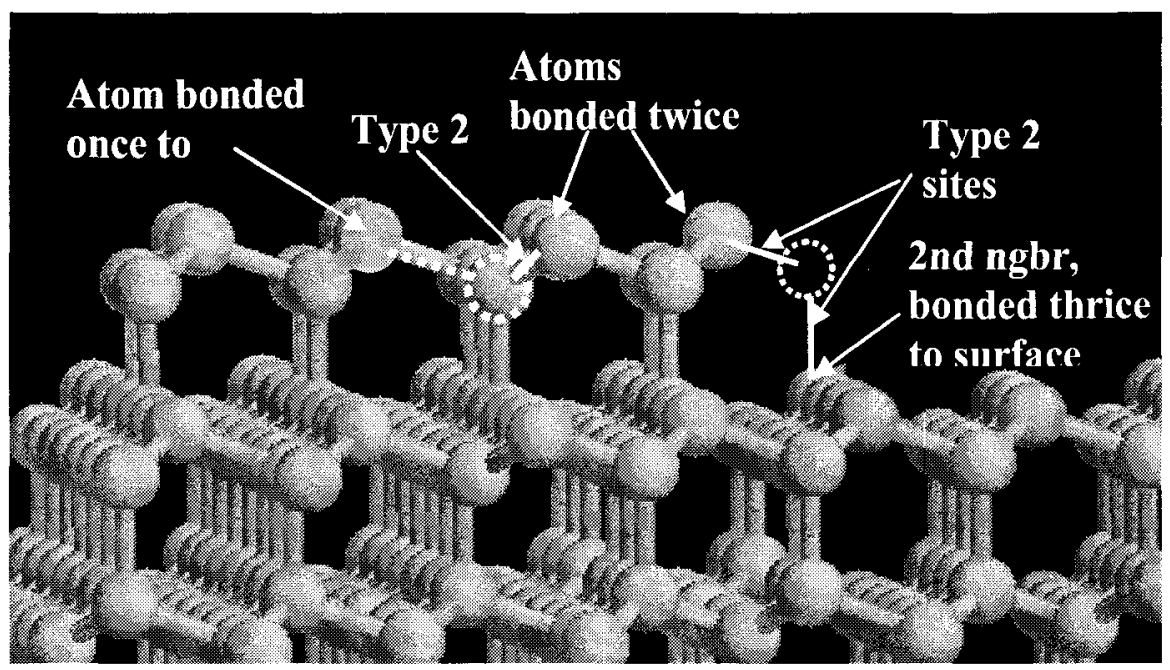

Figure 5.9 Ball and stick model of Type-2 sites on an atom bonded twice to the surface, also known as kink-sites.

Type 3: These sites are available on atoms bonded thrice to the surface (stable $\{111\}$ type of sites), which have a second neighbor that is bonded only once. This type of site initiates the formation of diamond $\{110\}$ (see fig. 5.10). Only adsorption occurs on this site.

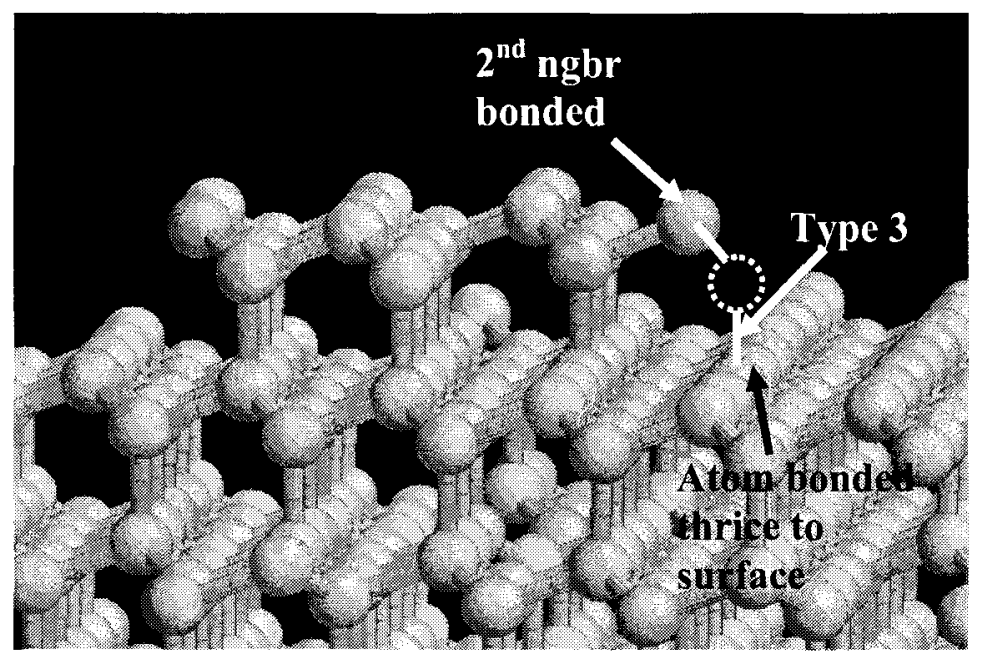

Figure 5.10 Ball and stick model of Type- 3 site found on $\{111\}$ surface, which could give rise to $\{110\}$ type surface after adsorption. 
Type 4: When two atoms bonded once to the surface are in close proximity to each other such that they are $2^{\text {nd }}$ neighbors, they will now be able to get a fixed orientation (either eclipsed or staggered) by an additional adsorption event. Their respective free bonds that make them $2^{\text {nd }}$ neighbors are now type 4 sites (see Figure 5.11). Adsorption on these sites makes a 3 atom bridge on $\{111\}$ surface. For most times, this three-atom bridge is capable of becoming a cubic step or hexagonal step (proper stacking or stacking fault respectively). However, for the simulations carried out in this work, only cubic stacking is considered. The atoms connected to these sites also have a tendency to get desorbed.

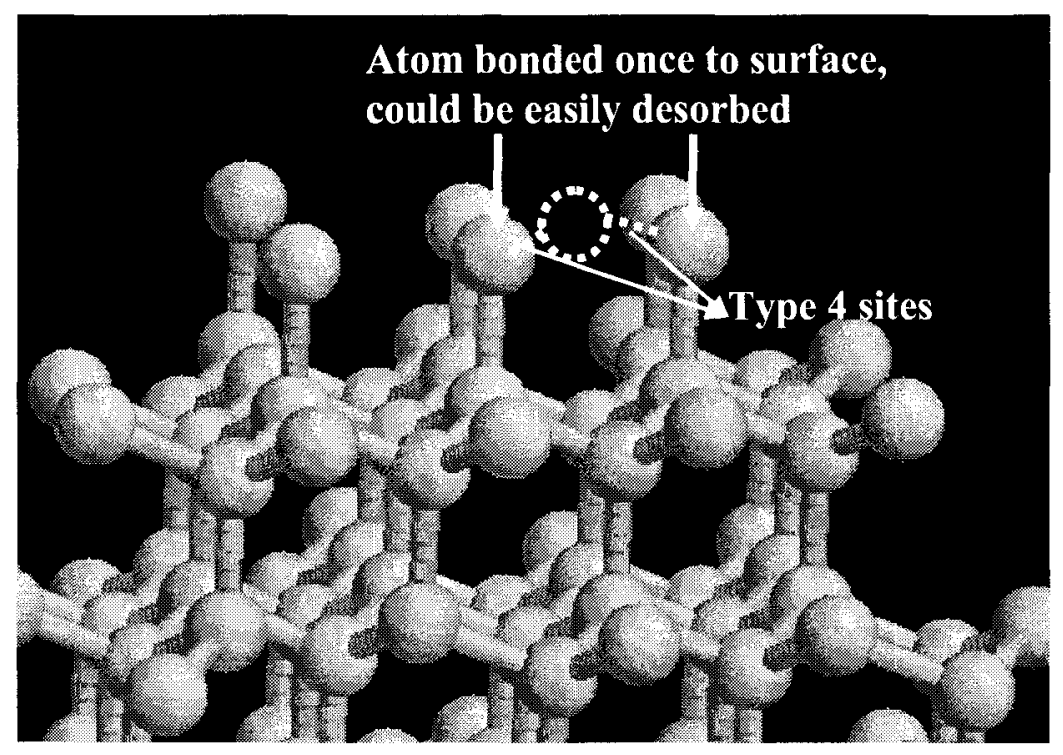

Figure 5.11 Ball and stick model of Type-4 site, which is found on singly-bonded atoms having second neighbors.

Type 5: These types of sites are found on stable $\{111\}$ planes. A site on an atom bonded thrice that has a third neighbor, which is bonded once to the surface is called a 
type 5 site. Adsorption on this type of site leads to the formation of sites of type 4 , in which two singly bonded atoms come at second neighbor distances. Only adsorption can occur at these types of sites. See Figure 5.12.

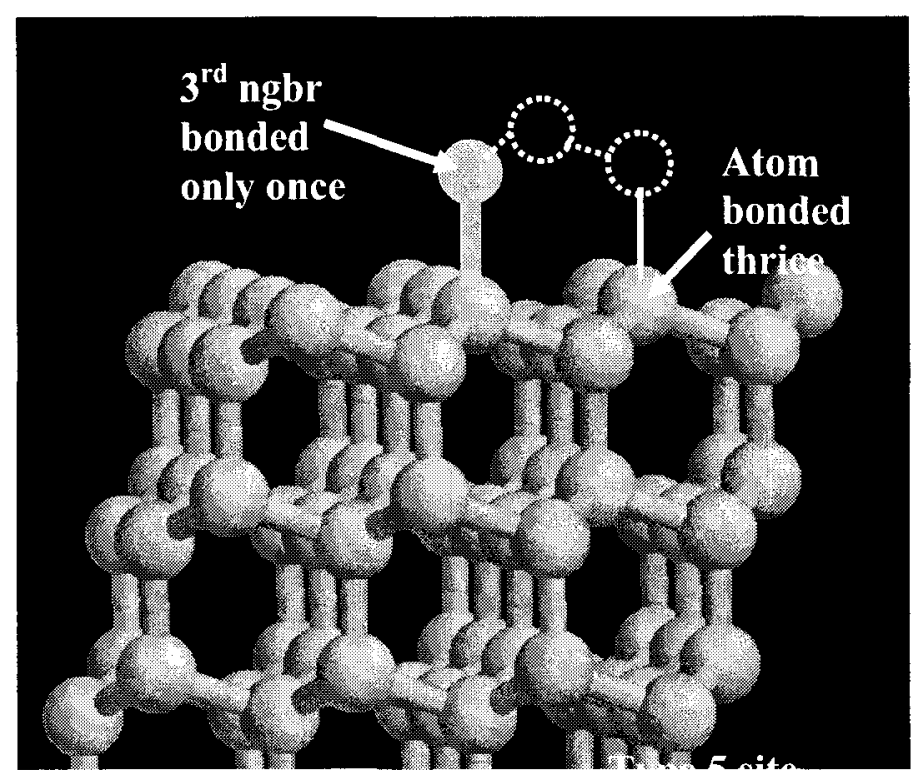

Figure 5.12 Ball and stick model of Type- 5 site, which is found on atoms bonded thrice to the surface.

Type 6: These sites are found either on atoms bonded twice or thrice to the surface with a third neighbor that is bonded more than once to the surface. Adsorption on this type of site is necessary to propagate the steps. These sites on triply bonded atoms are also found only on diamond $\{111\}$ surface, while those on doubly bonded atoms are found on $\{100\}$ surface. See Figure 5.13. 


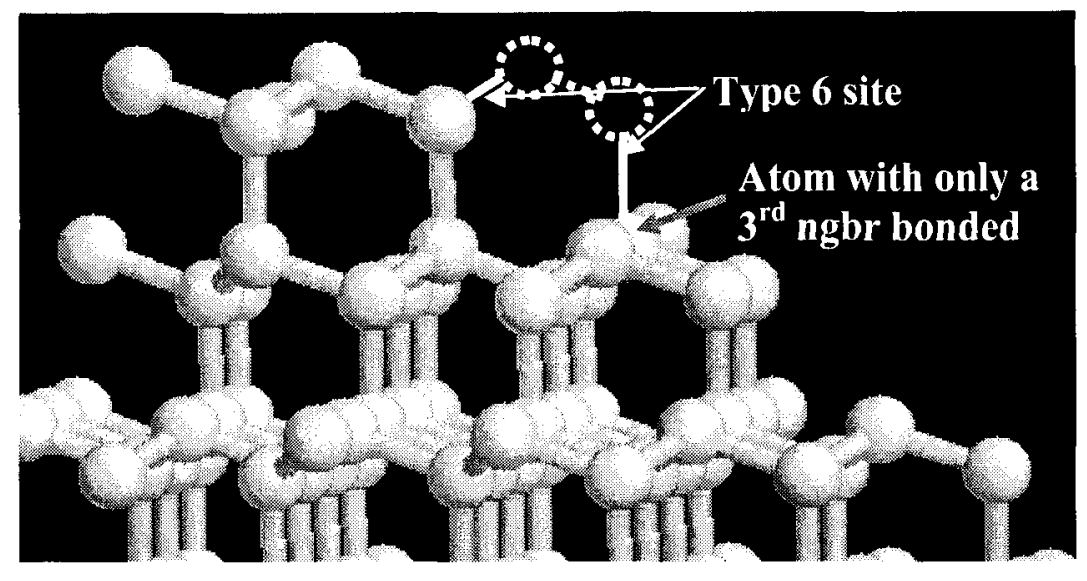

Figure 5.13 Ball and stick model of Type- 6 site, which is found on an atom bonded twice or thrice to the surface.

Type 7: This type of site is found on a doubly or triply bonded carbon atom with no $2^{\text {nd }}$, or $3^{\text {rd }}$ neighbors. They are strictly sites for adsorption. The adsorbed atoms when find a neighbor can lead to step nucleation. See Figure 5.14.

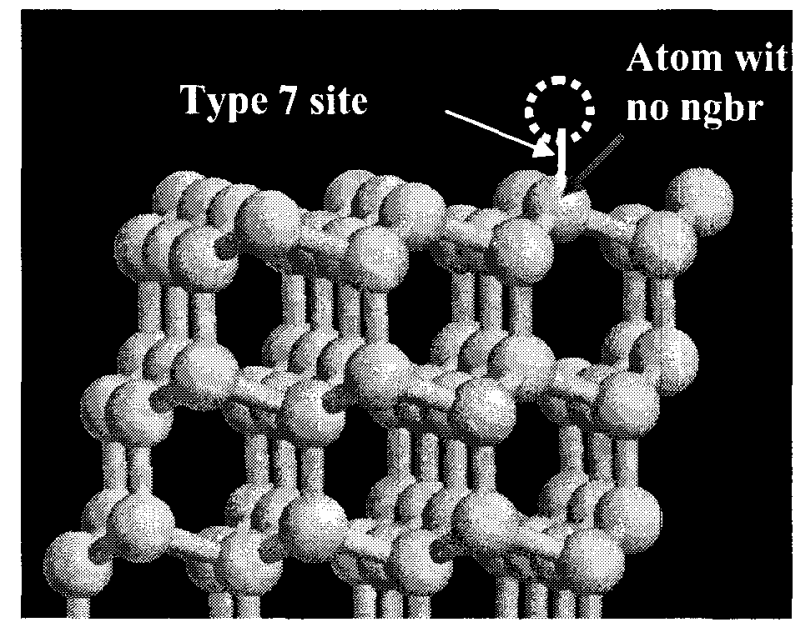

Figure 5.14 Ball and stick model of Type-7 site, which is found on singly or doubly bonded atoms with no first or second neighbors present. 


\subsubsection{Reaction rate constants}

Adsorption of single carbon species and desorption of surface atoms are termed as reactions. A rate constant is defined for each of these reactions ( $\lambda$ if adsorption, $\mu$ if desorption). Each of these reactions are assumed to be first order and irreversible. The $\lambda s$ and $\mu$ s selected for these simulations are arbitrary numbers and can be compared in ratio to realistic rate constants used in prior work [106-109]. The list of reactions with the assumed rate constants (arbitrary numbers) are listed in Table 5.1.

Table 5.1 Site-specific surface reactions and the range of rate constants used in the KMC simulations

\begin{tabular}{|l|l|l|}
\hline No. & Site-specific reaction & Range of rate constant \\
\hline 1. & Site $1 \rightarrow$ Desorption & $\mu_{1}: 1-20$ \\
\hline 2. & Site $\rightarrow$ Adsorption & $\lambda_{2}: 1-20$ \\
\hline 3. & Site $\rightarrow$ Desorption & $\mu_{2}: 0.01-0.2$ \\
\hline 4. & Site $3 \rightarrow$ Adsorption & $\lambda_{3}: 1-10$ \\
\hline 5. & Site $4 \rightarrow$ Adsorption & $\lambda_{4}: 1-5$ \\
\hline 6. & Site $4 \rightarrow$ Desorption & $\mu_{4}: 1-20$ \\
\hline 7. & Site $5 \rightarrow$ Adsorption & $\lambda_{5}: 1-5$ \\
\hline 8. & Site $6 \rightarrow$ Adsorption & $\lambda_{6}: 1-10$ \\
\hline 9. & Site $7 \rightarrow$ Adsorption & $\lambda_{7}: 1-10$ \\
\hline
\end{tabular}




\subsubsection{Algorithm}

The methodology used in the previous simulations [97] was similar to that used in Ref. 164. The algorithm of this scheme is as follows (Scheme 1):

1. A site is picked up at random.

2. The type of site is determined based on the neighborhood of parental atom, i.e. second neighbor and third neighbors of the parental atoms. The fractions of each type of sites are determined.

3. Depending on the site (for sites of type 4, 5 and 6 only), adsorption or desorption event is decided by a probability (for adsorption $=\lambda /(\lambda+\mu)$; for desorption $=\mu /(\lambda$ $+\mu)$ ). Based on this, the probability of the event is calculated as:

$$
\frac{m_{i} T_{i \rightarrow j}}{\left(\sum_{k \neq i} m_{i} T_{i \rightarrow k}+\sum_{i \neq k} m_{k} T_{k \rightarrow i}\right)}
$$

$m_{i}$ is the number of sites of type $i$ at time $t, T_{i \rightarrow j}$ is the conditional probability of transition from $i$ to $j$ per unit time. $\sum_{k \neq i} m_{k} T_{k \rightarrow i}$ is the total rate of transitions possible at time $\mathrm{t}$ from all other sites to site type i. Similarly $\sum_{k \neq i} m_{i} T_{i \rightarrow k}$ is the total rate of transitions possible from site type $\mathrm{i}$ to all other sites. Between the time $\mathrm{t}$ and $\mathrm{t}+\delta \mathrm{t}$, only a single transition event is possible. $T_{i \rightarrow j}$ becomes $\lambda_{i}$ for adsorption event and $\mathrm{T}_{\mathrm{j} \rightarrow \mathrm{i}}$ becomes $\mu_{\mathrm{j}}$ for a desorption event.

4. A random number is generated and is compared with the calculated probability. Based on the random number, the decision of the event (adsorption or desorption) is made.

5. The increment of time $\delta t$ is given as: 


$$
\left(\sum_{k \neq i} m_{i} T_{i \rightarrow k}+\sum_{i \neq k} m_{k} T_{k \rightarrow i}\right)^{-1}
$$

Scheme 2: In order to use detailed site-specific chemistry, another scheme described in reference [106] was used. The algorithm proceeds as follows (Refer to Fig. 5.15):

1. The site-fractions of each type of site are calculated.

2. The rate of reaction i (total $\mathrm{N}$ reactions) is calculated.

3. The probability of reaction i occurring is given as $P_{i}=\frac{r_{i}}{R}$, where $R=\sum_{j=1, N} r_{j}$

4. A scale of these rates is prepared. A random number is generated and if this number happened to be in the range of $\mathrm{P}_{\mathrm{i}}$, then reaction $\mathrm{i}$ would be chosen.

5. Once the type of reaction is decided, the type of site is picked up randomly from the cluster of all sites present at that instance on the surface.

6. Time is incremented dynamically and stochastically as $\delta t=-\ln (X) / R$, where $\mathrm{X}$ is a random number. 


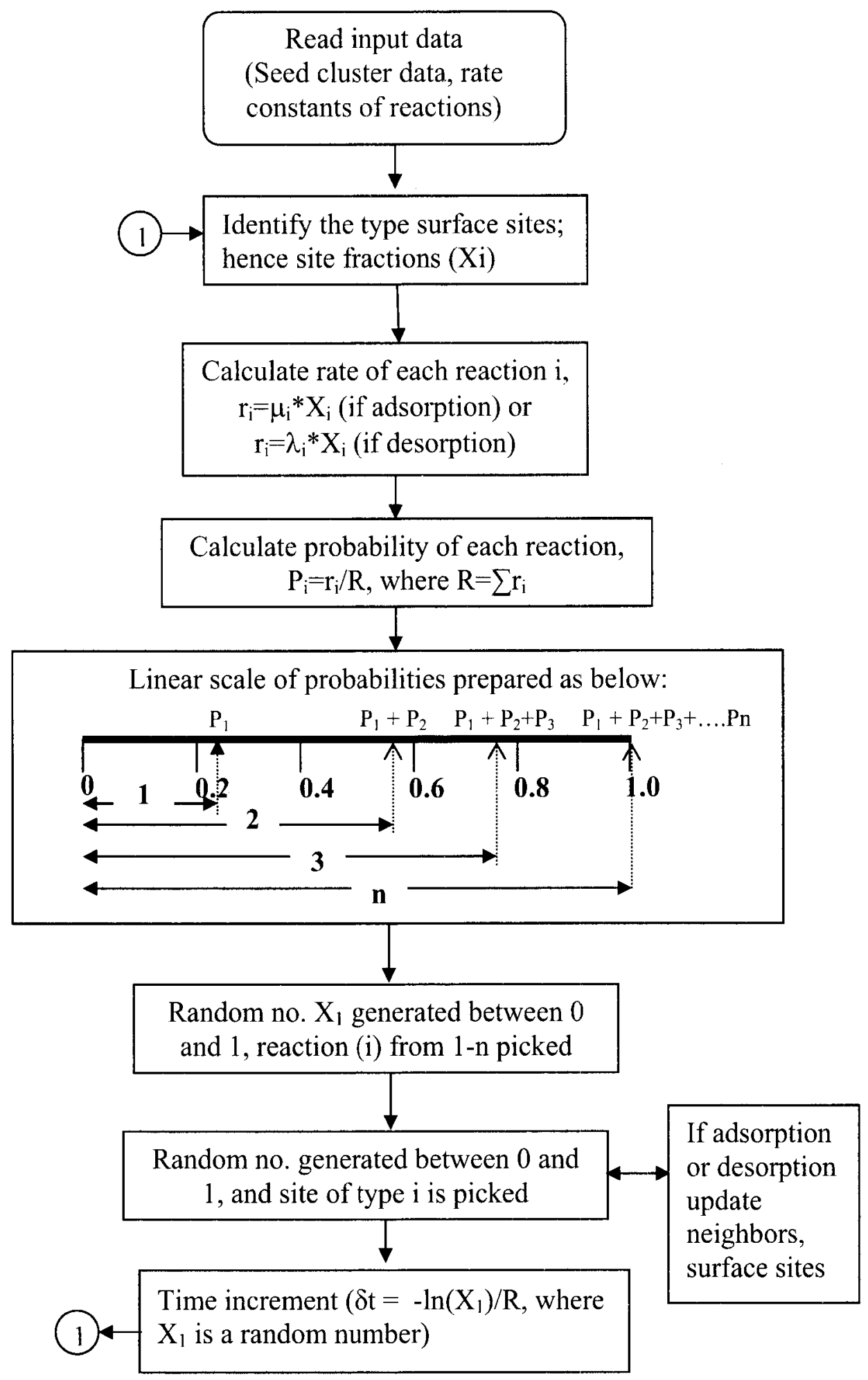

Figure 5.15 Algorithm for the KMC simulations used in this study. 
These simulations are stopped when a desired number of atoms are reached. Since simulations are done on a closed surface, cyclic boundary conditions are not necessary to account for edges. In each of the schemes, after atoms are adsorbed or desorbed, the corresponding matrices for atom bonding and sites fractions need to be updated continuously.

Both these schemes were tried for the different simulations. Comparing the two schemes, scheme 2 was chosen for the following reasons:

1. The results of the two schemes were not too different. The reason could be that Scheme 1 is always at equilibrium at any size of the crystal. Hence it would maintain the same morphology independent of size, except for very small number of atoms $(<1000)$. While, the Scheme 2 gives the actual time evolution of the crystal as it grows in three dimensions.

2. The rate constants used in the simulations have a greater control on the morphology in scheme 2. The reason being that in the Scheme 1, in reactions where adsorption and desorption occur, the event is decided by probability of $\lambda /(\lambda+\mu)$ or $\mu /(\lambda+\mu)$. While in Scheme 2 , it just depends on the rate of that reaction.

3. The time calculated by Scheme 2 give the true growth rate of the crystal. Hence one can make plots by using different rate constants.

This KMC methodologies mentioned above does not require the prior knowledge of actual rate constants of the reactions (which are very difficult to obtain for gas-surface reactions). Arbitrary numbers can be used and depending on the results of the simulations (the final morphology); they could be changed to obtain the desired 
morphology. Thus, in a way, this method gives a good estimate of the ratio of rate constants. However, the growth rate information gathered in this method would not be correct. If even one of the rate constants were known (for instance addition of a methyl radical to a $\{111\}$ surface), the rest of the rate constants would also be known with sufficient accuracy, and hence the growth rate information could be derived.

The program code was written in Fortran 77 and was run on a SGI ONYX supercomputer. The shape of the simulated crystal was visualized using RasMol $2.7^{\circledR}$ (developed by Roger Sayle) and Cerius ${ }^{2 \mathbb{B}}$ (Accelrys, CA) software packages.

\subsubsection{Time increment}

The KMC algorithm used (Scheme 2) is an $\mathrm{N}$-fold way approach. In this method one event occurs compulsorily at each time increment, and the rate of the reactions are chosen from probabilities, the time increment with each even $(\delta t)$ is also dynamic and stochastic [106].

$\delta t=-\frac{\ln (\xi)}{\sum_{i=1}^{M} r_{i}}$,

Where $\xi$ is a random number uniformly distributed in the range $(0,1)$ and the denominator is the sum of the rates of all of the events that can occur at the simulation step for which $\delta t$ is evaluated. When the reaction rates are fast, $\delta t$ becomes low, and when the reaction rates are low, $\delta t$ is fast. 


\subsubsection{Seeds used in the simulation}

KMC simulations start with a seed cluster containing 10-20 carbon atoms and are allowed to grow individual crystals of any desired number of atoms. The evolution of perfect decahedral, perfect icosahedral, and dodecahedral shapes was successfully illustrated by a Kinetic Monte Carlo (KMC) simulation scheme using single adatom adsorption and desorption and starting with a seed cluster containing two and three stacking errors using scheme 1 [97]. However, the purpose of the study undertaken was to understand the reasons for formation of star-shape in decahedron, dimples (in stardecahedron and icosahedron) and additional facets along the twin boundaries. The number of stacking faults was pre-decided in the simulations (as shown in Figure 5.16) to generate the desired 5-fold or twenty-fold crystal, since the purpose was to understand the effect of kinetics on some of the features like dimples, additional $\{111\}$ facets, etc. All the KMC simulations for five-fold twins (whether decahedron, star-decahedron or dodecahedron) were performed using the same seed cluster containing two stacking faults at different $\{111\}$ planes (Figure 5.16b), while simulations for all twenty-fold crystals (icosahedron or dimpled icosahedron) were carried out on seed with three stacking faults (Figure $5.16 \mathrm{c})$.
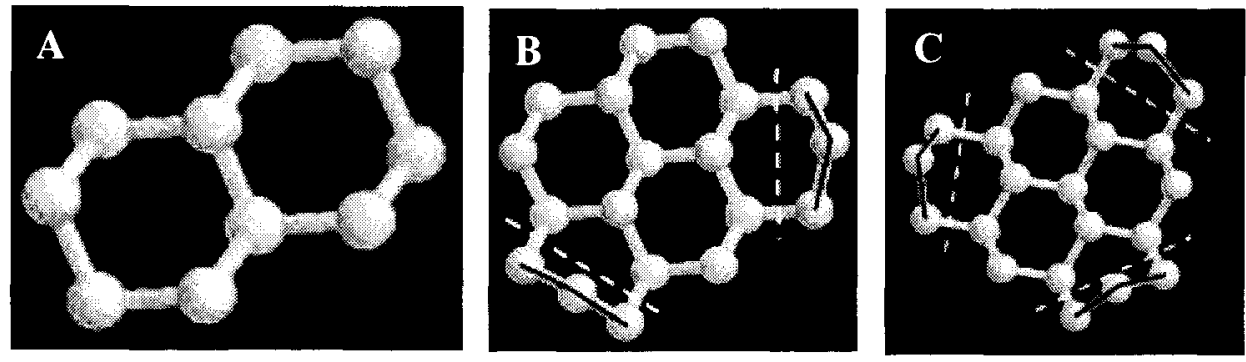

Figure 5.16 Ball and stick model of (a) untwinned seed, (b) seed with two stacking errors, and (c) seed with three stacking errors on different $\{111\}$ planes. 
Changing the size of the seeds, for instance, increasing the distance between the stacking faults governed the final size of the twinned crystal, but did not change the final overall morphology.

\subsubsection{Results on morphologies of computer grown crystals}

All the simulations were carried out for at least 5000 atoms. And in many cases, the ones with favorable results (morphology matching experimentally observed ones) were carried out up to 20,000 atoms. The simulated crystals containing 20,000 atoms correspond to a physical size of $\sim 10 \mathrm{~nm}$. Steady state, in terms of both shape and growth kinetics, was generally reached in these simulations when the crystal size reached 1000 atoms (as will be illustrated in further sections). For the size of the clusters being simulated, these simulations run relatively very fast. However, as the size of the cluster increases, the simulations slow down for processes like calculations of types of sites, picking up the selected type of site, etc. The reason for this slowing down is because the size of the matrices that contain all the bonding and neighbor information increases. The real time taken by the simulation depends on the probability of adsorption and cluster size. For a crystal size of 10,000 atoms, it varies from five hours (at highest adsorption probabilities) to more than 24 hours (at low adsorption probabilities).

\subsubsection{Simulations using a single adatom adsorption/desorption}

In the first set of simulations carried out, there was not much site selectivity used. The only 4 types of sites considered were Type 1, Type 4, Type 5 and the rest clubbed to Type 6. No selectivity was used in adsorption or desorption at these sites $\left(\lambda_{4}=\lambda_{5}=\lambda_{6}\right.$ 
$=\lambda$, and $\mu_{1}=\mu_{4}=\mu$ ). For five-fold crystals with these sites using high desorption to adsorption ratios, i.e. $\mu / \lambda=5-20$, decahedral crystals with $10\{111\}$ facets and negligible $\{100\}$ facets resulted from the simulations. The result is shown in Figure 5.17. With a relatively high ratio of adsorption to desorption rate constant ratios, i.e. $\lambda / \mu=5-100$, the same simulations resulted in a dodecahedral crystal with $10\{111\}$ facets and $5\{100\}$ facets as shown in Figure 5.18.

When the five-twinned $\{111\}$ planes, with $\mathrm{sp}^{3}$ bond angles, try to form a closed structure, a $7.5^{\circ}$ mismatch occurs $\left(360-5^{*} 70.5^{\circ}=7.5^{\circ}\right)$. This angle misfit leads to an incomplete closure of five-fold crystals. High-resolution transmission electron microscopy (HRTEM) of five-fold twinned crystals showed that the misfit ( 7.5 degrees) is primarily accommodated near one or two twin boundaries by creating additional dislocations [165] and other higher order stacking faults [166]. Hence, the simulated crystals (Figure $5.17,5.18$ ) are consistent with the HRTEM observations with only one or two twin boundaries accommodating the misfit during growth. 

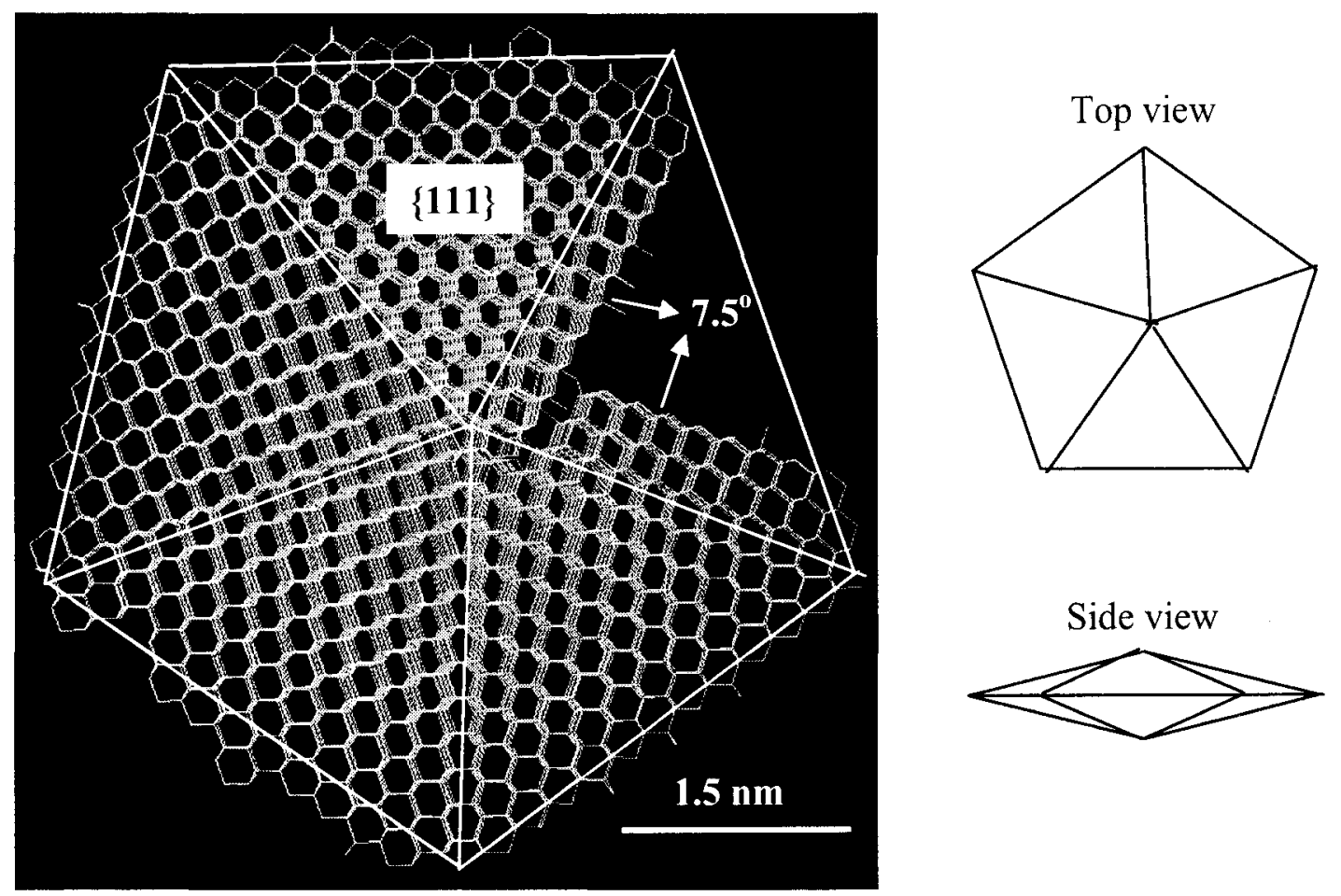

Figure 5.17 Wire-frame model of simulated decahedral crystal (5000 atoms) with single adatom adsorption/desorption kinetics $(\mu / \lambda=20)$ (approx. size $6 \mathrm{~nm}$ ).

The simulations on a seed cluster without any stacking error, using the same ratio of rate constants $(\mu / \lambda=5-20, \lambda / \mu=5-100)$ produced an octahedron and cubo-octahedron respectively. This is intuitive from the fact that high adsorption of single methyl radicals leads to quicker formation of steps. If the step flow cannot keep pace with the step nucleation, bunching of steps occurs, which gives rise to $\{100\}$ facets. On the other hand, if step nucleation were a slow event, there would be sufficient time for the already formed step to flow across the crystal. Thus, the evolution of a decahedral shape to a dodecahedral shape for crystals seems to follow a similar relationship as that of octahedral to cubo-octahedral. This suggested that the $\alpha$ factor (defined in Section 2.5) 
for a decahedral crystal would be the same as for an octahedral crystal $(\alpha=3.0)$ and that for a cubo-octahedral crystal would be the same for a dodecahedral crystal $(\alpha=1.5)$ [62].
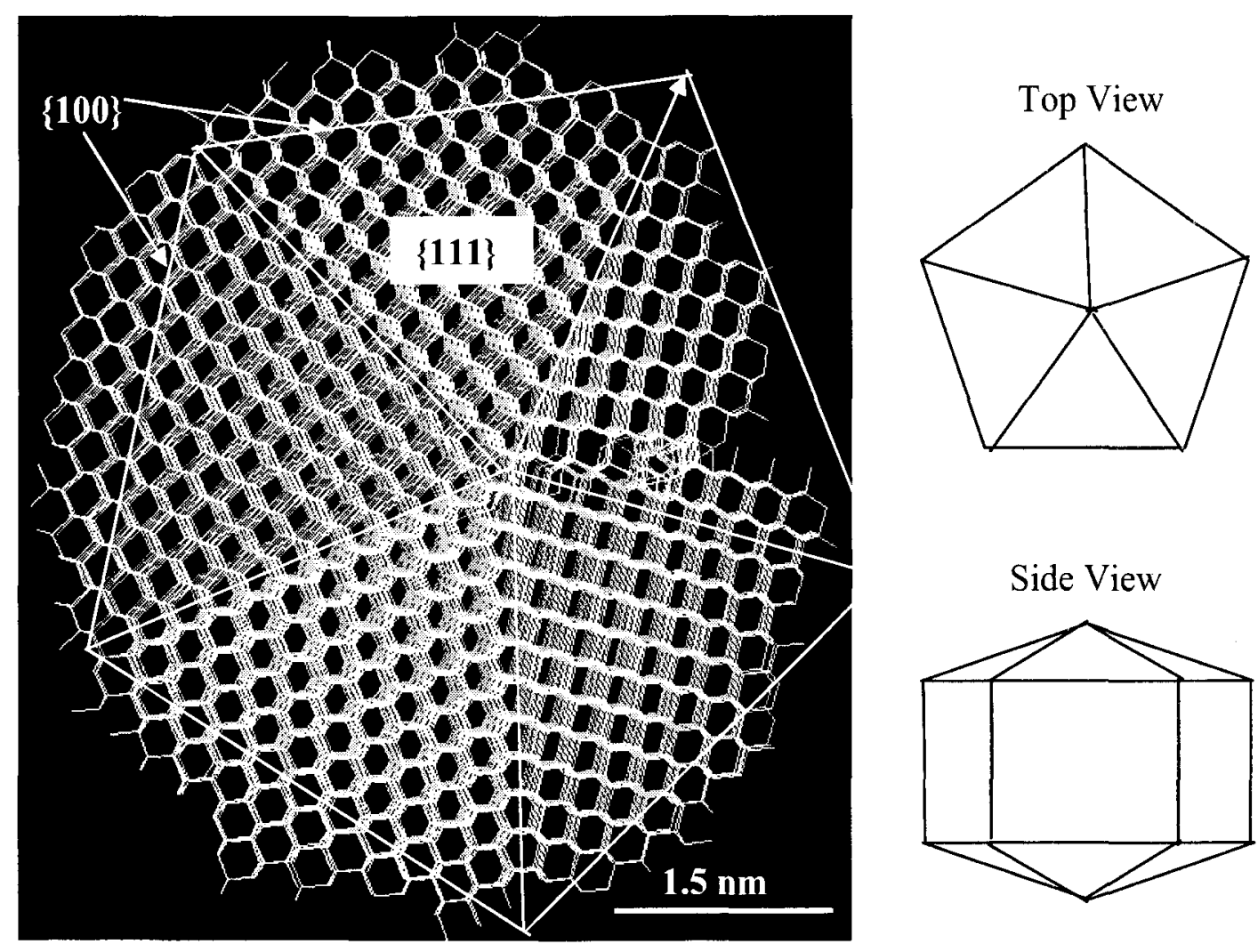

Figure 5.18 Wire-frame model of simulated dodecahedral crystal (5000 atoms) with single adatom adsorption/desorption kinetics $(\mu / \lambda=0.1)$ (approx. size $6 \mathrm{~nm}$ ). (There are 5 additional $\{100\}$ facets at the sides giving a thickness to the crystal).

Using the seed Fig. 5.16c, with high desorption to adsorption ratio $(\mu / \lambda=5-20)$ a twenty-fold icosahedral crystal was obtained (Figure 5.19). This crystal had all twelve 5fold corners pointed, and did not have any dimples. This result also agrees with that obtained from ref [97]. 

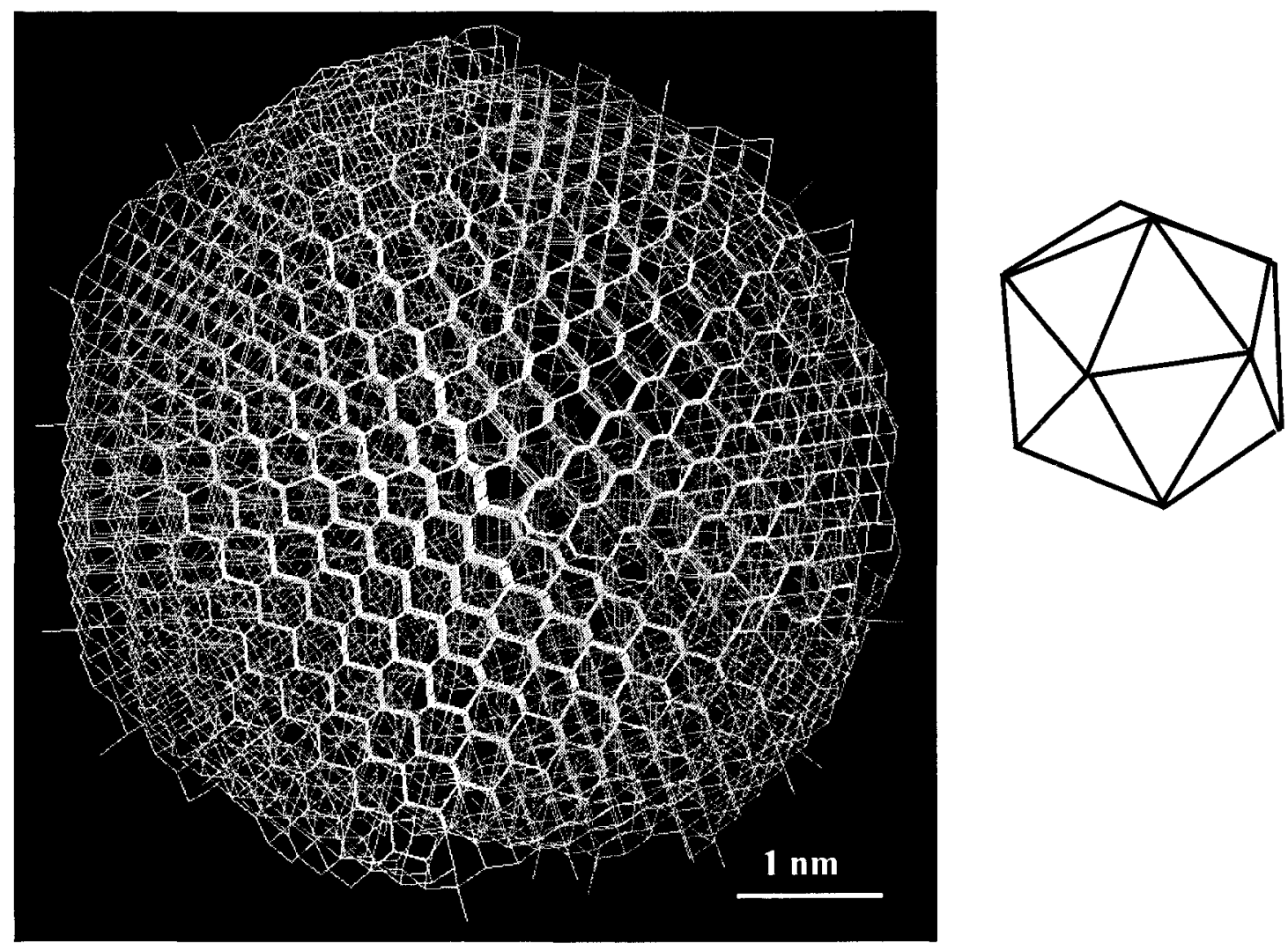

Figure 5.19 Wire-frame model of simulated icosahedral crystal (5000 atoms) with single adatom adsorption/desorption kinetics $(\mu / \lambda=20)$ (approx. size $4 \mathrm{~nm})$.

Appendix 2 shows the simulation of a five-fold crystal after 200 atoms is deliberately made to acquire an additional stacking fault, which then changes to an icosahedral crystal. This is similar to the experimental phenomena reported in section 5.2.1.4. There were few parameters that could be manipulated in these simulations $(\lambda / \mu)$, which did not explain the reason for the presence of additional $\{111\}$ faceting and dimples that appeared in star-decahedral and icosahedral crystals. 


\subsubsection{Simulations with finite etching of doubly bonded atoms}

The very appearance of a star-decahedral crystal with dimples and additional $\{111\}$ facets along twin boundaries, makes it appear as if the removal of atoms seem to be responsible for these feature, making it star-shaped. Hence, $\mathrm{KMC}$ simulations were performed using finite desorption rate constant for etching of surface sites. The types of sites considered were now Type 1, Type 2, Type 4, Type 5 and Type 3, 6 and 7 clubbed together (from now on referred to as Type 3-6-7). Thus, there were 5 main types of sites identified. No selectivity was used in adsorption at any of these sites (i.e. $\lambda_{2}=\lambda_{4}=\lambda_{5}=$ $\left.\lambda_{3-6-7}=\lambda\right)$. However, additional selective etching of site type 2 was included. Hence, $\mu_{1}$ $=\mu_{4}=\mu$ and $\mu_{2}$ were different. The simulations, over a range of desorption rate constant ratios for doubly bonded to singly bonded surface sites $\left(\mu_{2-3} / \mu=0.001-0.02\right)$, maintaining the same adsorption rate constants as $\left(\lambda_{2}=\lambda_{4}=\lambda_{5}=\lambda_{3-6-7}=\lambda=1-4\right)$, yielded starshaped decahedral crystals with truncated $\{100\}$ facets. Figure 5.20a shows the stardecahedral crystals grown by simulations. It should be noted that in order to achieve these simulation results, the allowed etching of doubly bonded surface sites was not selective (i.e. it occurred equally irrespective of the number of neighbors the site had). Figure $5.20 \mathrm{~b}$ shows the simulated star-decahedron with atom filling. Here, one can see that there seems to be no dimple at the center. 

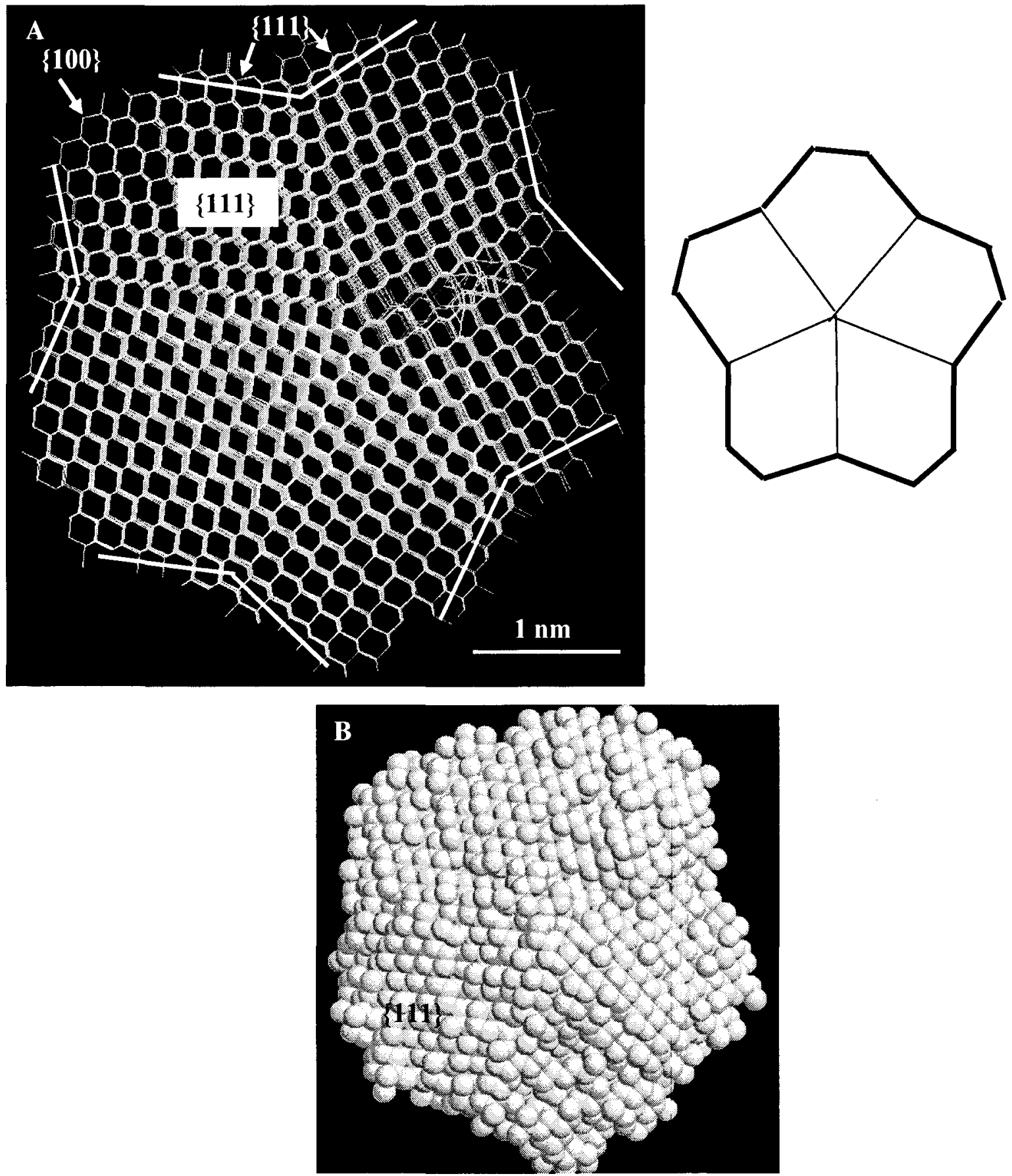

Figure 5.20 (a) Wire-frame and (b) space-filled model of a simulated star-dodecahedral with single adatom adsorption/desorption and doubly bonded atom etching $(\lambda / \mu=0.4$, $\mu_{2}=0.2$ ) (approx. size $5 \mathrm{~nm}$ ). 
The etching of doubly bonded sites was used previously to explain the triangular etch pits observed in naturally occurring samples as well as in the liquid-phase wetsolution etching of diamond crystals $[167,168]$. However, the theoretical probability for thermal desorption of doubly bonded surface sites is $9-10$ orders of magnitude less than that for the desorption of singly bonded adatoms. Gas phase selective etching of isolated doubly bonded atoms was used in reference [169] to explain slow growth rates of diamond $\{100\}$, while the etching of doubly bonded atoms at terraces and step edge sites was calculated to be unappreciable. The etching used in these simulations was not selective. Thus, the true value of $\mu_{2-3}$ is much smaller than the one used in the simulations. In this regard, the results from the above simulations are only suggestive of a chemical means of etching such surface sites. This type of etching, if allowed, would require the critical nucleus for step nucleation on a $\{111\}$ surface to contain at least four adatoms instead of three adatoms.

\subsubsection{Simulations with increased site-specific chemistry}

In order to simulate dimples as well as well-defined additional $\{111\}$ facets, it was clear that a more site-specific chemistry needed to be used. In these simulations now, all the 7 types of sites defined in section 5.3.1.2 were used. The rate constants were varied for each type of site. Etching was carried out only for singly bonded methyl radicals i.e. the only desorption events that occurred were on site type 1 and type 4 ( $\mu_{1}$, $\left.\mu_{4}\right)$. The adsorption varied on all the other site types $\left(\lambda_{2}, \lambda_{3}, \lambda_{4}, \lambda_{5}, \lambda_{6}, \lambda_{7}, \lambda_{8}\right)$. A range of rate constants was tried for the simulations. 
In general, a higher desorption vs. adsorption ratio for single adatoms resulted in $\{111\}$ faceted crystals. This is consistent with the previous simulations mentioned in section 5.3.2.1. Figure 5.21(a) shows the results for a star-decahedral crystal. The rate constants used for this simulation are $\mu_{1}=5, \mu_{4}=5, \lambda_{2}=10, \lambda_{3}=1, \lambda_{4}=1, \lambda_{5}=1, \lambda_{6}=5, \lambda_{7}=1$. A clearly noticeable dimple is seen at the center of the star-decahedral. The solid-model shown in Figure 5.21b shows the dimple appearing at the center, with clearly defined $\{111\}$ facets. The $\{111\}$ facets are identified easily by the arrangement of atoms in a triangular symmetry. There exists a clear similarity between the simulated stardecahedron and experimentally grown crystal shown in Figures 5.2a. The dimple did seem to get buried during some stages of growth; this could be simulating the actual process of growth of star-decahedral, in which the dimples would change in size. Figure 5.22a represents a large star-decahedral crystal of about 19,500 atoms $(\sim 10 \mathrm{~nm})$. This simulation took several days of running. The dimples seemed to have buried in this large crystal (Figure 5.22b), but clear additional $\{111\}$ faceting still continue to appear. 

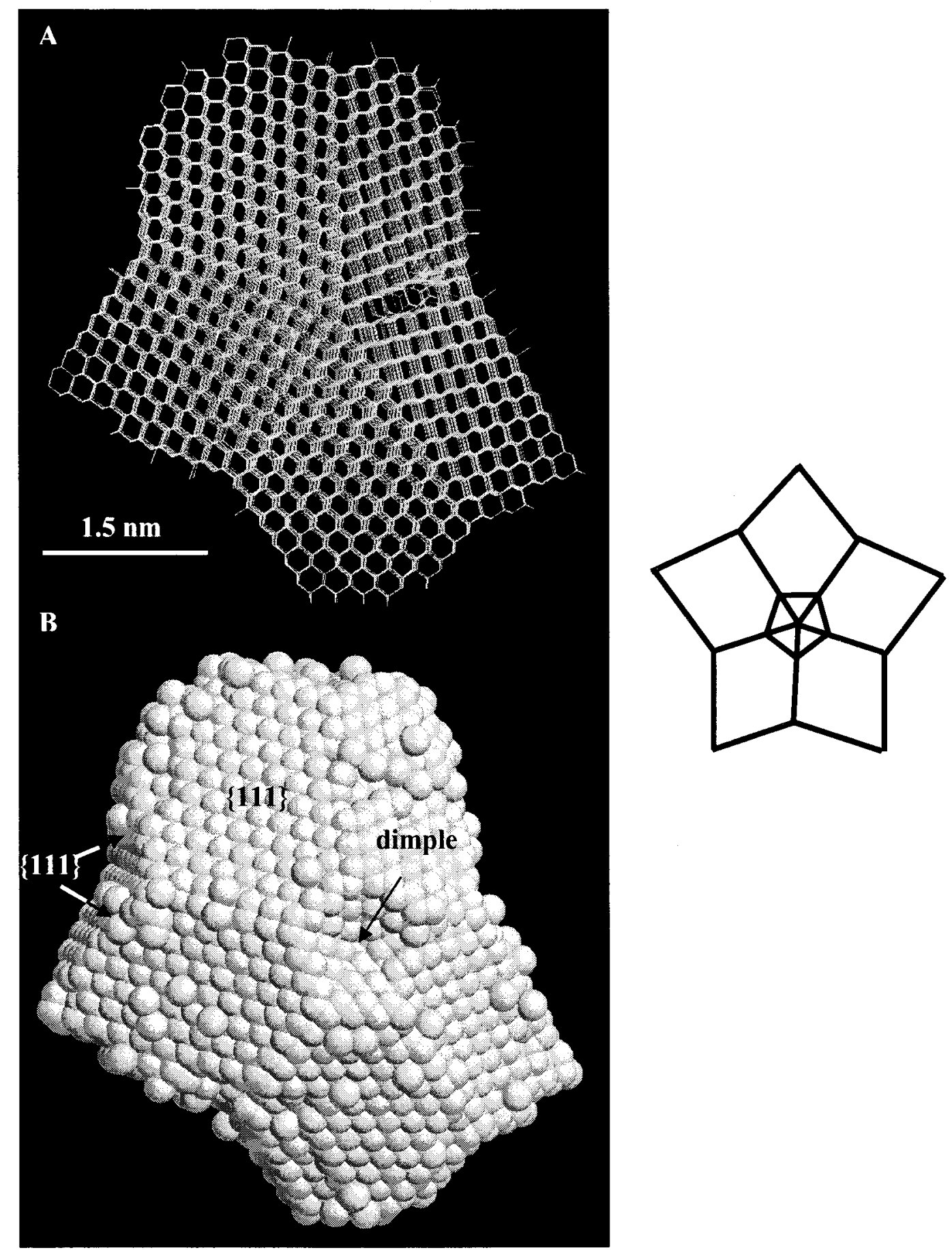

Figure 5.21 (a) Wire-frame and (b) space-filled model of a simulated star-decahedral crystal containing 7000 atoms with a prominent dimple using rate constants $\mu_{1}=5, \mu_{4}=5$, $\lambda_{2}=10, \lambda_{3}=1, \lambda_{4}=1, \lambda_{5}=1, \lambda_{6}=5, \lambda_{7}=1$ (approx. size $6 \mathrm{~nm}$ ). 

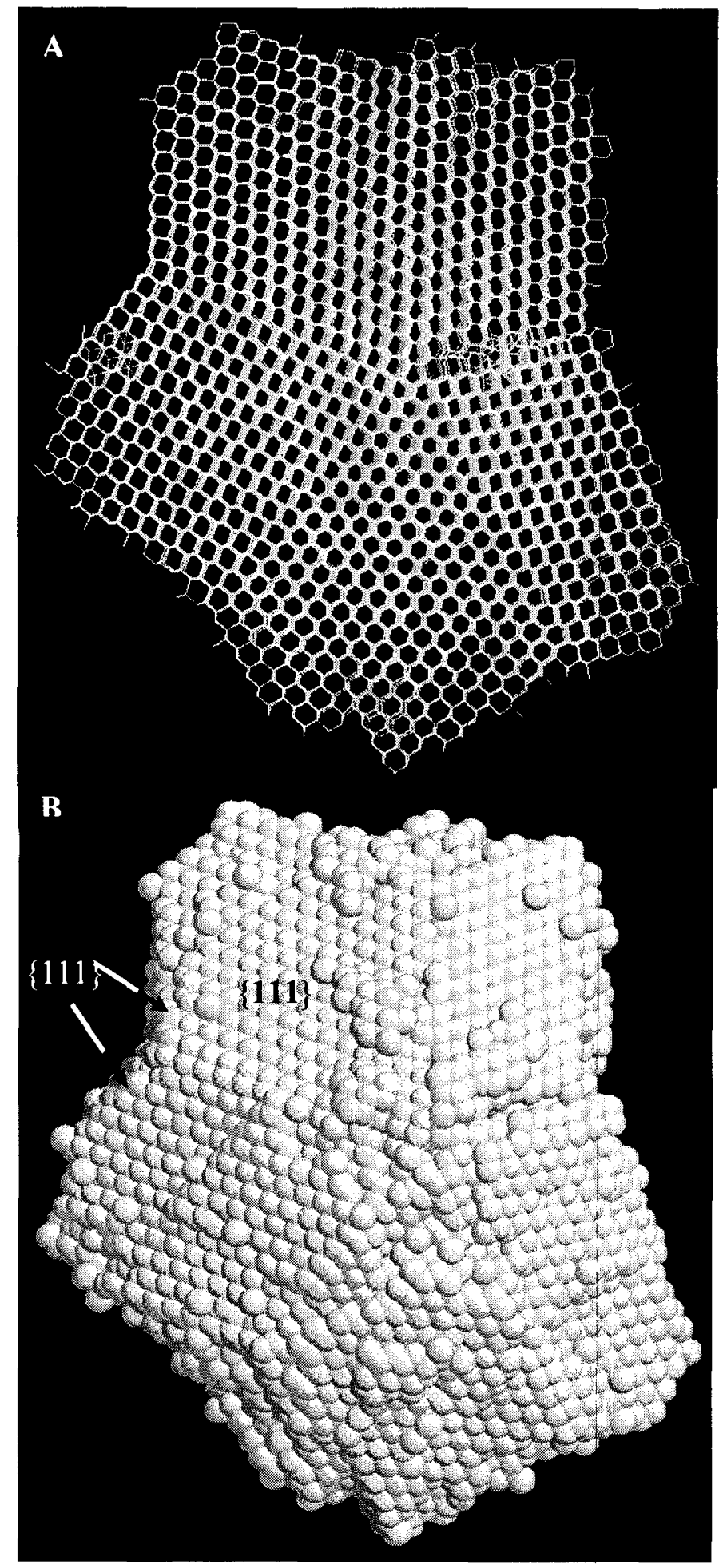

Figure 5.22 (a) Wire-frame and (b) space-filled model of a long-term simulation of a stardecahedral crystal with 19500 atoms (approx. size $10 \mathrm{~nm}$ ). 
From Figure 5.21, it may seem apparent that the planes enclosing the dimples are $\{11\}$, which is partially consistent with the AFM observations reported in section 5.2.2. However, as mentioned above, the dimples were partially filled during the simulations. Calculations of the angle, when the dimple is covered with one partial step leads to an increase of $29.7^{\circ}$, which increases the angle to $140^{\circ}$. Figure 5.23 shows a sequence of a dimple, which initially is completely bounded by $\{111\}$ (Figure 5.23a), and after growth of 500 additional atoms, the center etch pits fills up partially due to the growth of a monolayer (Figure 5.23b), increasing the angle to $140^{\circ}$. This result is again consistent with the AFM results reported in section 5.2.2, indicating at some instances the dimple planes could be high index planes. These figures can be compared to Figure 5.7 showing dimple in a real star-decahedral crystal.

It must be remembered that these simulations were performed at the scale of at least two orders of magnitude smaller than real size crystals. Hence, this partial filling of the etch pit could be visualized as few $\{111\}$ layers in the dimple developing incomplete steps, giving rise to a high index $\{115\}$ planes. 

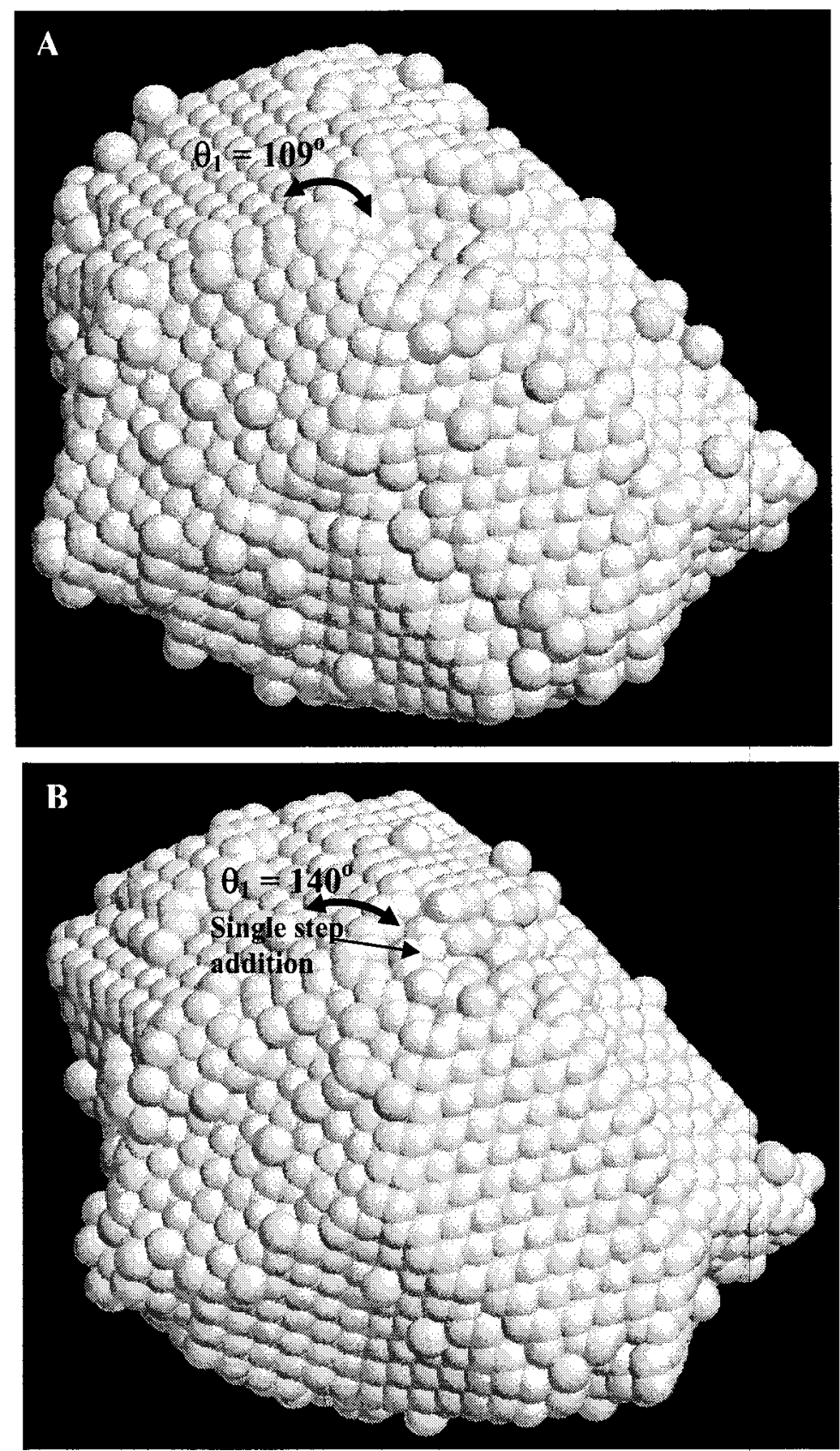

Figure 5.23 Space-filled model of a simulated growth sequence of a star-decahedral at (a) 7000 atoms with a prominent dimple and (b) 7500 atoms with dimple partially filled.

Simulations for a decahedron resulted with the rate constants $\mu_{1}=10, \mu_{4}=10, \lambda_{2}=5$, $\lambda_{3}=10, \lambda_{4}=1, \lambda_{5}=1, \lambda_{6}=10, \lambda_{7}=1$ is shown in Figure 5.24. As it is clear in the space-filled 
model (Figure 5.24b) there is no dimple or additional faceting in this crystal. Simulation for a dodecahedron is show in Figure 5.25 for rate constants $\mu_{1}=2, \mu_{4}=2, \lambda_{2}=2, \lambda_{3}=10$, $\lambda_{4}=2, \lambda_{5}=1, \lambda_{6}=1, \lambda_{7}=2$. The dodecahedral crystal has a thickness due to the additional $\{100\}$ facets. The dodecahedral crystal shows significant step bunching.

Thus, these set of rate constants and reactions seem sufficient enough to define conditions under which a star-decahedral, decahedral and do-decahedral crystals are obtained. 


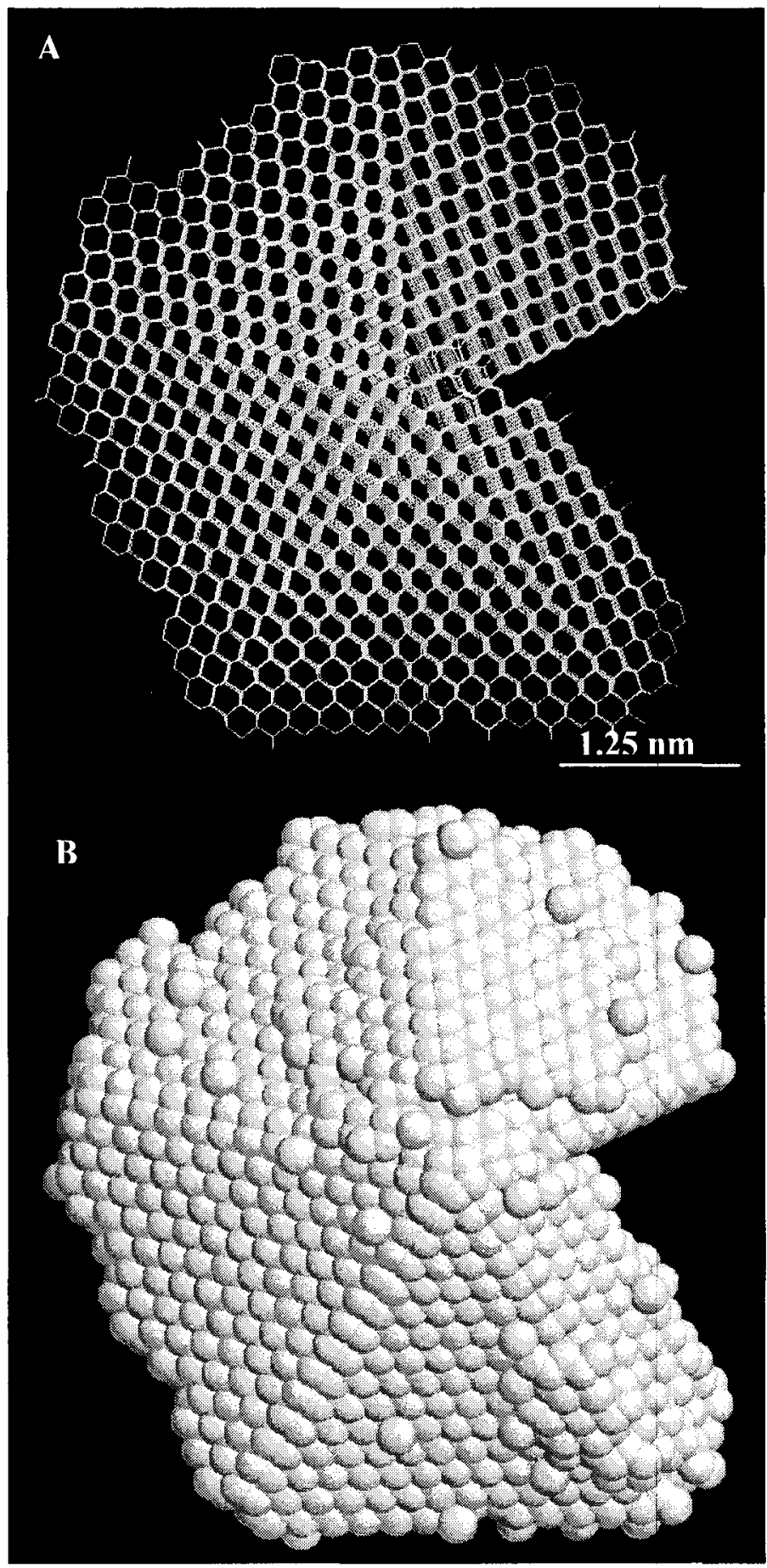

Figure 5.24. (a) Wire-frame model and (b) space-filled model of a simulated decahedral crystal containing 7000 atoms using rate constants $\mu_{1}=10, \mu_{4}=10, \lambda_{2}=5, \lambda_{3}=10, \lambda_{4}=1$, $\lambda_{5}=1, \lambda_{6}=10, \lambda_{7}=1$ (approx. size $6 \mathrm{~nm}$ ). 


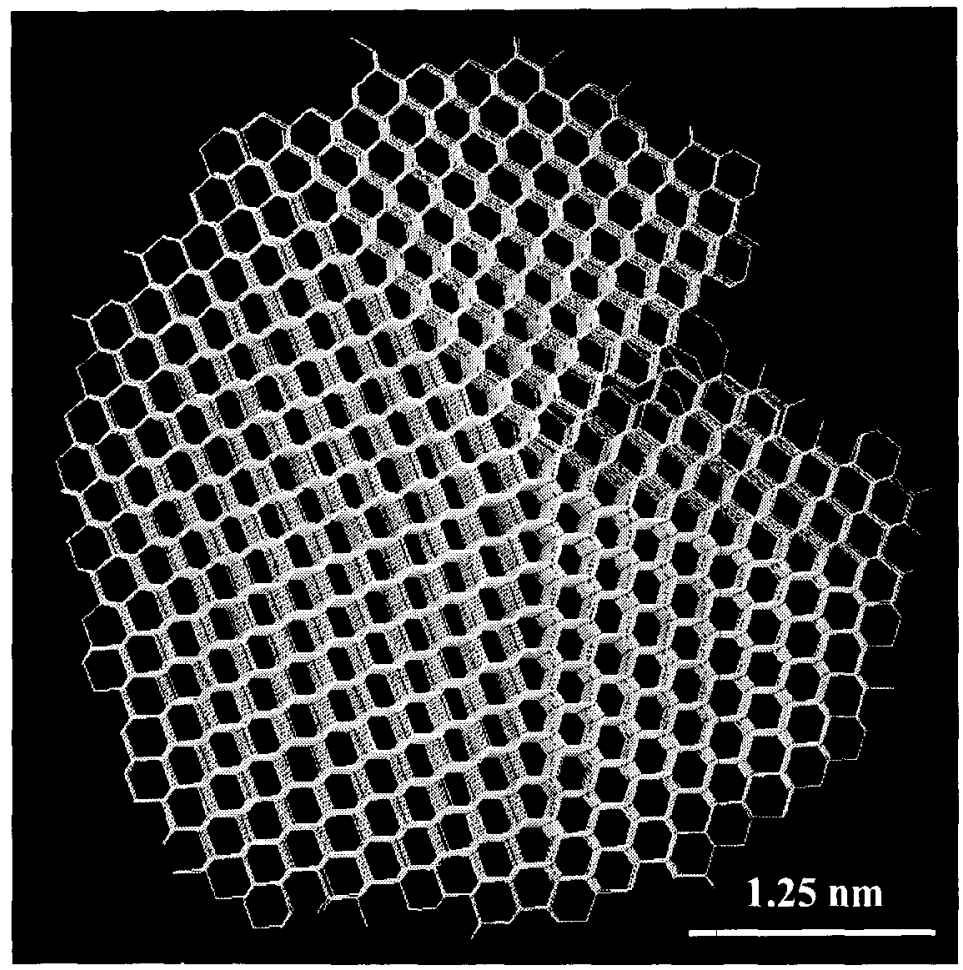

Figure 5.25 Wire-frame model of a simulated dodecahedral crystal containing 7000 atoms using rate constants $\mu_{1}=2, \mu_{4}=2, \lambda_{2}=2, \lambda_{3}=10, \lambda_{4}=2, \lambda_{5}=1, \lambda_{6}=1, \lambda_{7}=2$ (approx. size $5 \mathrm{~nm})$.

Using the rate constants that result in decahedral crystal on a seed with three stacking faults (Figure 5.16c) results in a perfect icosahedral crystal with no dimples at the twin centers (Figure 5.26a). Using the same conditions that gave rise to stardecahedral, but with a seed containing 3 staking faults, an icosahedral crystal with dimples is obtained (Figure 5.26b). It must be noted that the dimples get buried after a few thousand atoms, because these become preferential sites for adsorption than other sites. The 7.5 angle mismatch of each five-fold center makes it difficult to complete the icosahedron in general, thus, it does not close up. 


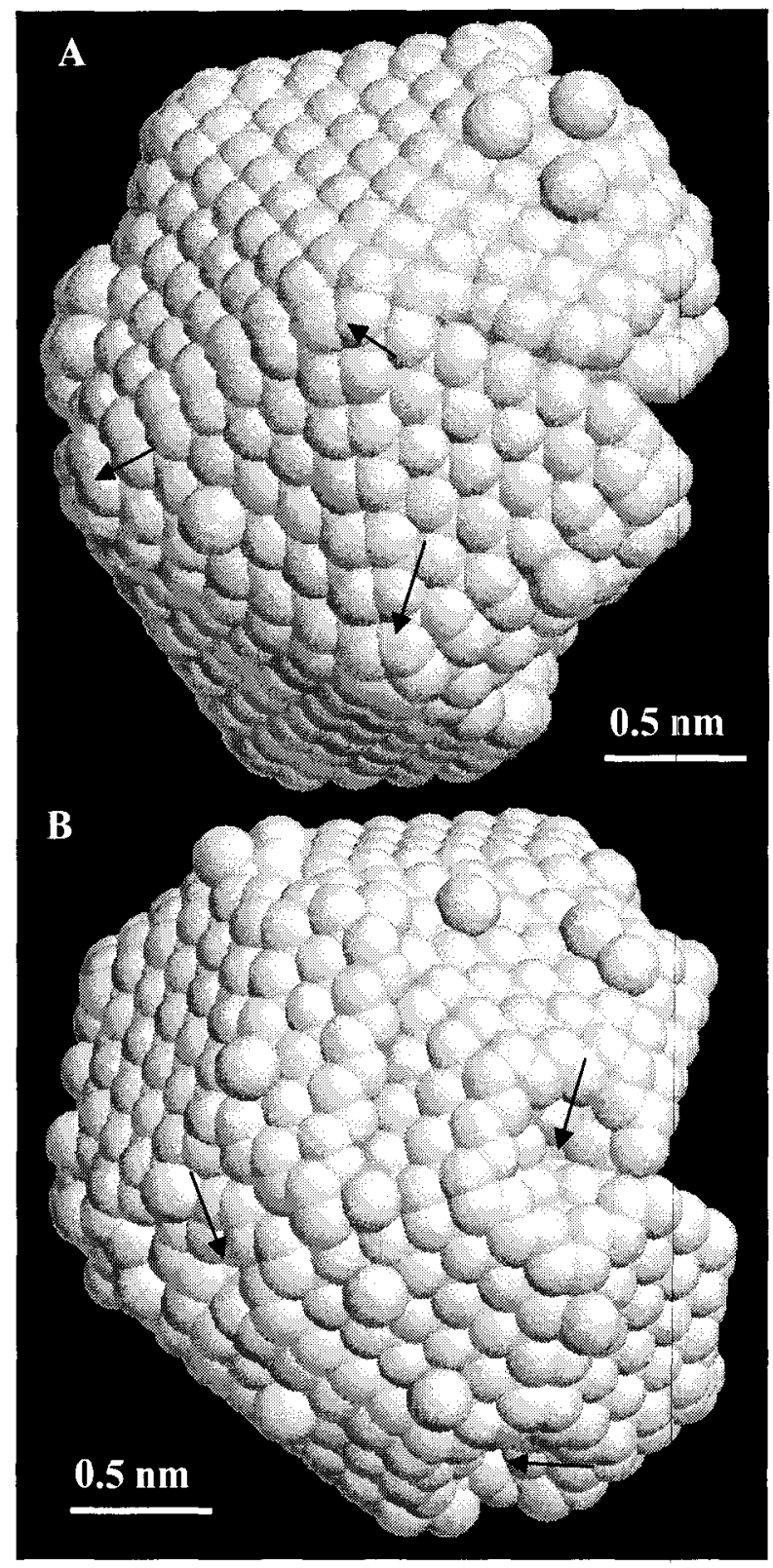

Figure 5.26 Space-filled model of (a) simulated pefect-icosahedaral (same rate constants as decahedral crystals) and (b) simulated dimpled icosahedron (same rate constants as star-decahedral). (Arrows indicate 5-fold twin centers). 


\subsubsection{Discussion on KMC results}

Among the three sets of KMC simulations used, the one described in section 5.3.2.3 with detailed site-specific chemistry seems to work best in explaining the variations of five-fold crystals (star-decahedral, decahedral, and dodecahedral), and twenty-fold crystals (icosahedral and dimpled icosahedral). The $\alpha$ factors for simulated star-decahedron and decahedron shown in Figure 5.21 and 5.24 were calculated. According to the definition, $\alpha=\sqrt{3} \cdot v_{100} / v_{111}$, (described in section 2.5) where $v_{100}$ and $\mathrm{v}_{111}$ are the velocities of the [100] and [111] directions. $\mathrm{v}_{100}$ and $\mathrm{v}_{111}$ are again given by the distance of the $\{111\}$ and $\{100\}$ facets from the center of the crystal divided by the time. Since the crystal is growing in 3-D, the time taken for $\{111\}$ and $\{100\}$ facets would be same and would cancel out. Hence, $\alpha=\sqrt{3} \cdot d_{100} / d_{111}$, where $\mathrm{d}_{100}$ and $\mathrm{d}_{111}$ are distances of the $\{100\}$ and $\{111\}$ from the center of the crystal, as indicated in the side view in Figure 5.27. For the simulated decahedral crystal this value was 2.94 and for simulated star-decahedral crystal this value was 2.9.

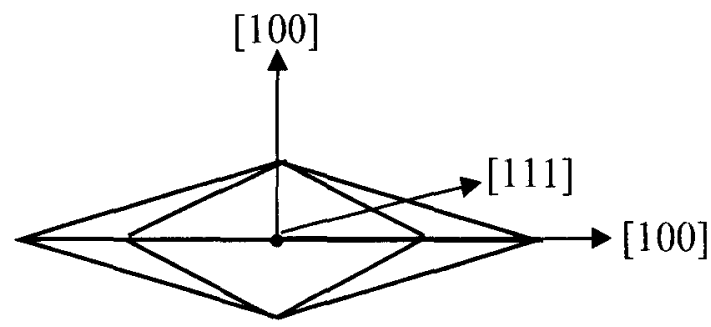

Figure 5.27 Schematic side-view of either a decahedral or star-decahedral crystal. (Square brackets indicate directions, which are perpendicular to the respective crystallographic planes). 
The same set of rate constants for decahedral and star-decahedral crystals were used on untwinned seeds (See Figure 5.16a) and simulations were carried out for the same number of atoms. These simulations resulted in the same type of octahedral crystals with a $\alpha$-factor close to 3 . It was thus apparent that there is no difference in $\alpha$ factor for star-decahedral or decahedral crystals. This is consistent with the fact that they are bound by $\{111\}$ facets.

The variation of crystal size with time is shown in Figure 5.28. The growth kinetics, after steady state, followed the expected relationship for attachment kinetics limited growth, i.e., cluster volume $(\mathrm{N})$ was proportional to $\mathrm{t}^{3}$.

Appendix 3 shows the simulation sequence of the star-decahedral and decahedral crystal formation starting with the seed containing two stacking error till about 1000 atoms. In star-decahedral and decahedral crystals, in general, desorption of single adatoms is higher than adsorption $\left(\mu_{1}, \mu_{4}\right.$ is higher than $\left.\lambda_{4}\right)$. This condition is required for $\{111\}$ bound crystals. The main difference in growth conditions (simulations) of decahedral and star-decahedral crystals is the value of the rate constant $\lambda_{2}, \lambda_{3}$, and $\lambda_{6}$. The site type 2 as described in section 5.3.1.2 is a kink site. This site is partially responsible for step propagation (Figure 5.9). The site of type 6 is also responsible for step-propagation; however, it requires two-adatom events for step propagation (Figure 5.13). In fact, once the two consecutive atom additions occur at this site, it gives rise to kink sites (type 2 sites). Hence, this event can be called "kink-nucleation". 

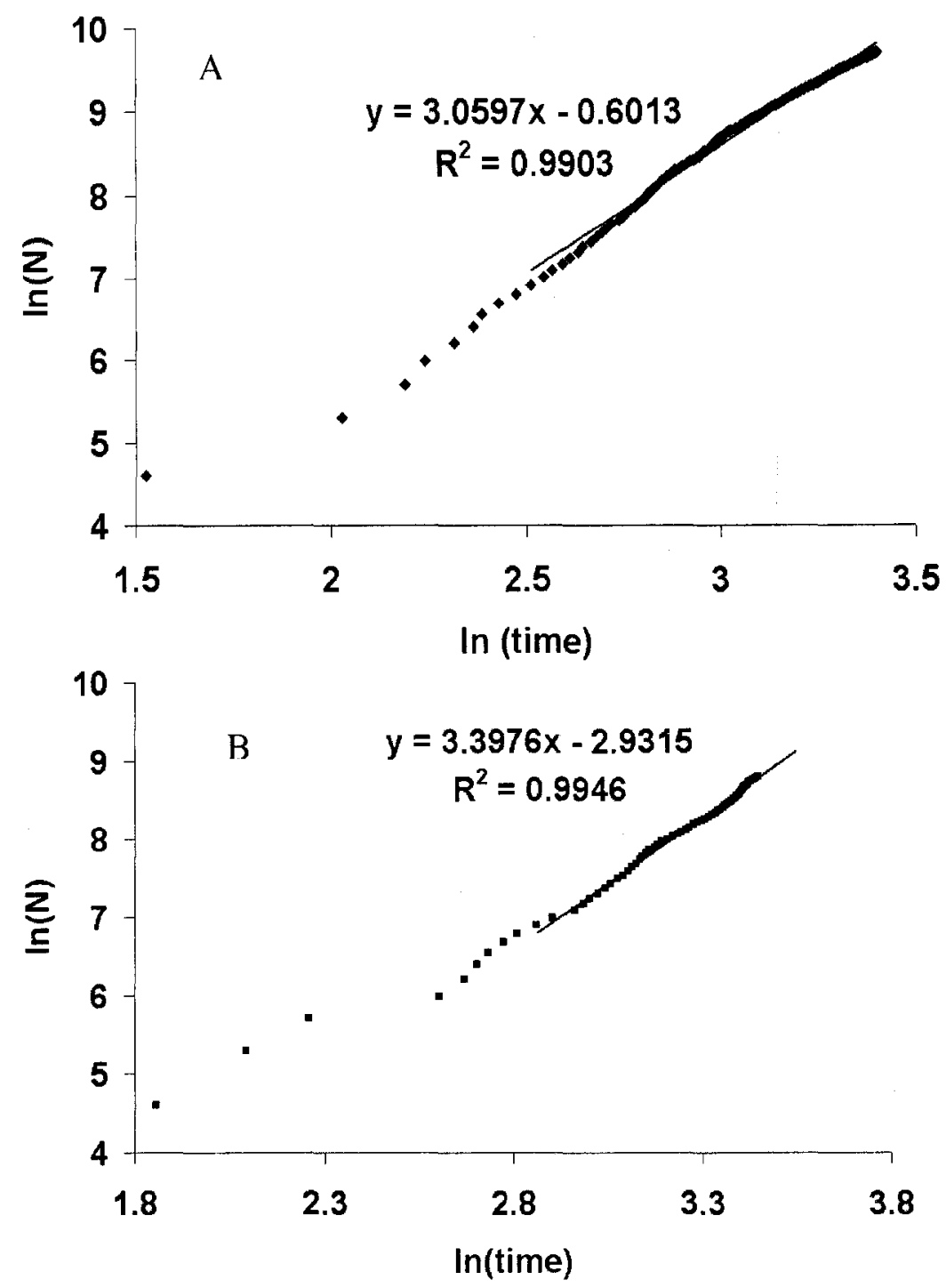

Figure 5.28 Logarithmic plot of number of atoms vs. time (arbitrary units) for (a) simulated star-decahedral crystal and (b) simulated decahedral crystal.

Under the lean growth conditions $\left(\left(\mu_{1}, \mu_{4}\right.\right.$ is higher than $\left.\lambda_{4}\right)$, once a step nucleates on a stable $\{111\}$ facet, it will propagate throughout the crystal, only after that does the next step nucleation event occur. Under these conditions, step-propagation is an 
important event. In the case of star-decahedron, due to the low adsorption on site 6 , versus site 2, step propagation event (or kink nucleation event) is limiting, while kink propagation is a very fast event. Hence, as soon as the step propagates through site 6 , kinks are nucleated, and adsorption immediately occurs on these sites. As a result, these step-islands tend to bunch up in the form of triangles bounded by $\{111\}$ sites in a stardecahedral crystal (Figure 5.29).

In a decahedral crystal, since adsorption at type 6 site is faster than type 2 site, kink nucleation and kink propagation are at least equivalent to each other. This leads to the formation of a triangular $\{111\}$ facet, but they are inverted with respect to that in a star-decahedral crystals (Figure 5.30). The point to be noted is that adsorption on type 2 and type 6 sites (even if slow) permit step flow across a convex twin plane. 


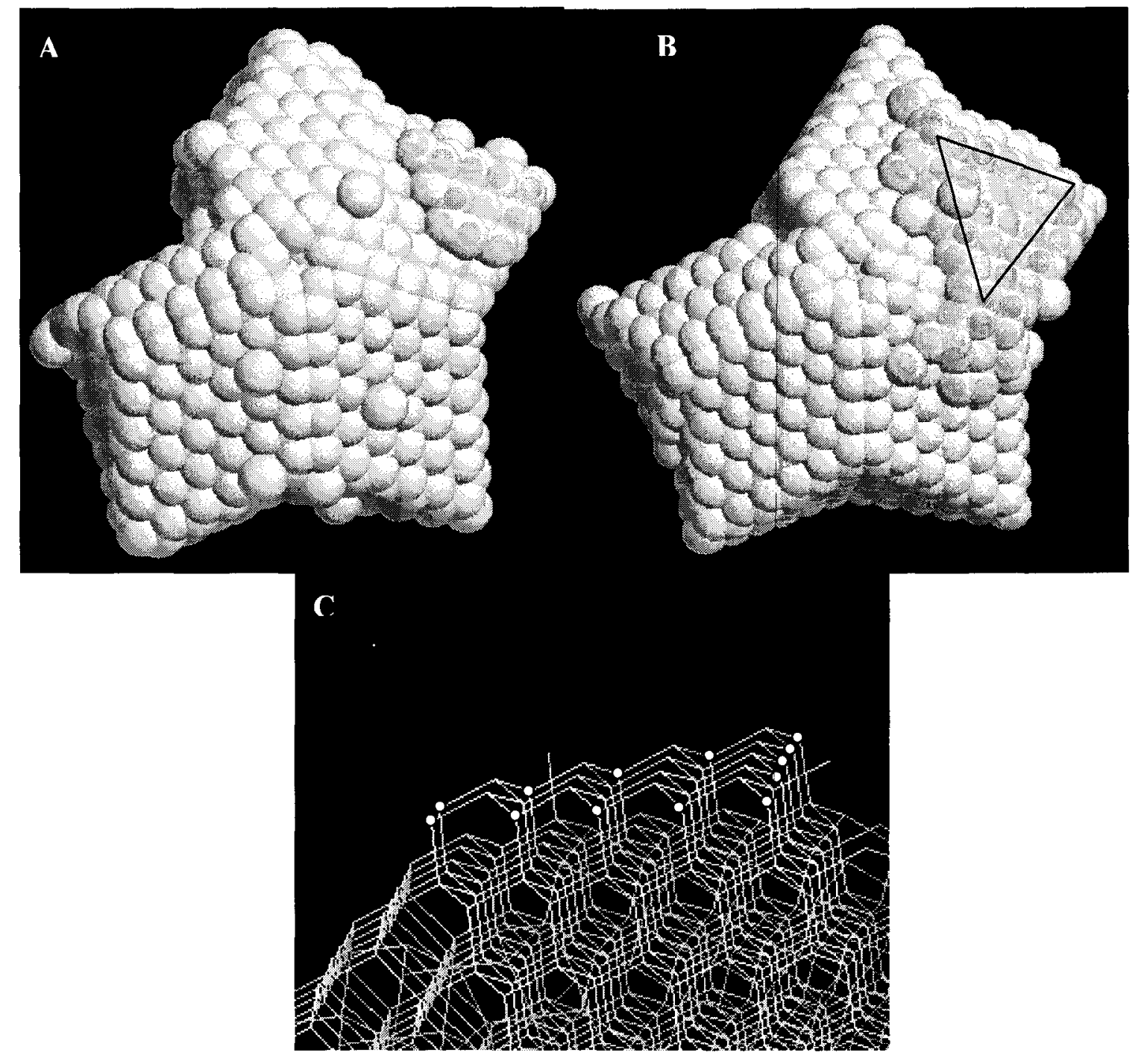

Figure 5.29 Simulated step propagation sequences on a star-decahedral crystal at different sizes: (a) 1900 atoms and (b) 2000 atoms. Wire-frame model illustrating the bonding nature of the step. (Type-6 sites are marked as white circles). 


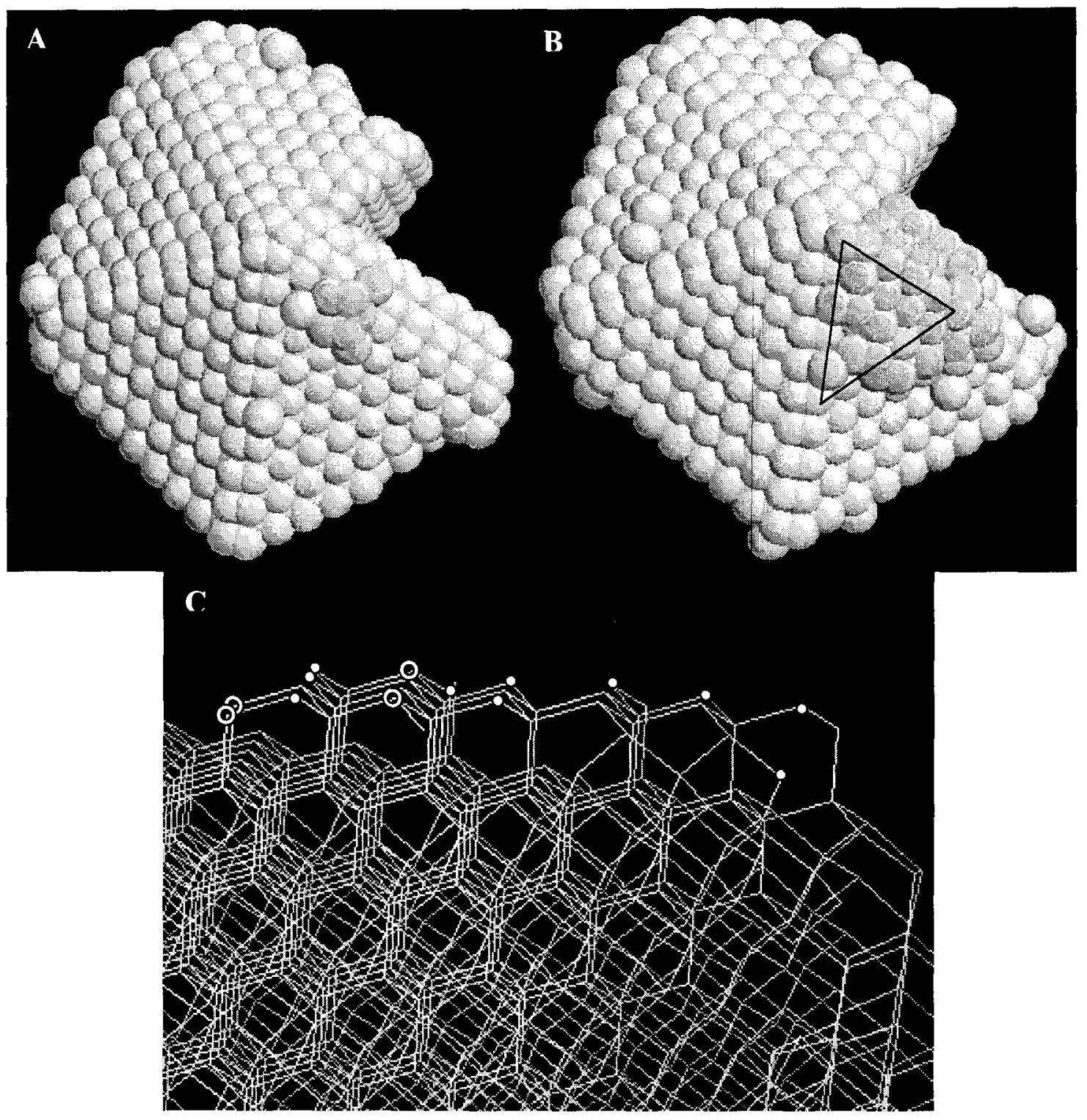

Figure 5.30 Simulated step propagation sequences on a decahedral crystal at different sizes: (a) 1700 atoms and (b) 1800 atoms. Wire-frame model illustrating the bonding nature of the step. (Type- 2 sites are marked as filled circles and type- 6 sites are marked as open circles). 
For star-decahedron, the value of $\lambda_{3}$ is almost $1 / 10$ the the value in a decahedron. As indicated in section 5.2.2, type 3 is a site occurring on a triply bonded atom which has a second neighbor that is bonded only once to the surface. In a twinned crystal, nucleation at the twin boundaries requires adsorption on this type of a site. As shown in Figure 5.31, once adsorption of a single adatom occurs at the twin center, to form a stable step, just a second adatom is required on a triply bonded atom site (which is a type 3 site). On the other hand, a step nucleation on a $\{111\}$ plane requires a 3 -atom cluster, i.e. adsorption on a type 4 site. Once a stable step is formed at both, the twin plane or $\{111\}$ plane, it propagates immediately because of adsorption reactions on type 2 and type 6 sites, and due to the fact that step-propagation step is faster than step nucleation. Hence, if adsorption is slowed on site of type 3 , it would be difficult to nucleate a step at the twin boundary. The consequence of this is additional $\{111\}$ facets along the twin planes and additional facets also where the 5 twin planes meet at the pentagonal center of the stardecahedral (i.e. a dimple). Thus, the reduction on adsorption on type 3 and type 6 site are both responsible for the formation of additional $\{111\}$ facets along the twin boundary and dimples in a star-decahedral crystal. 


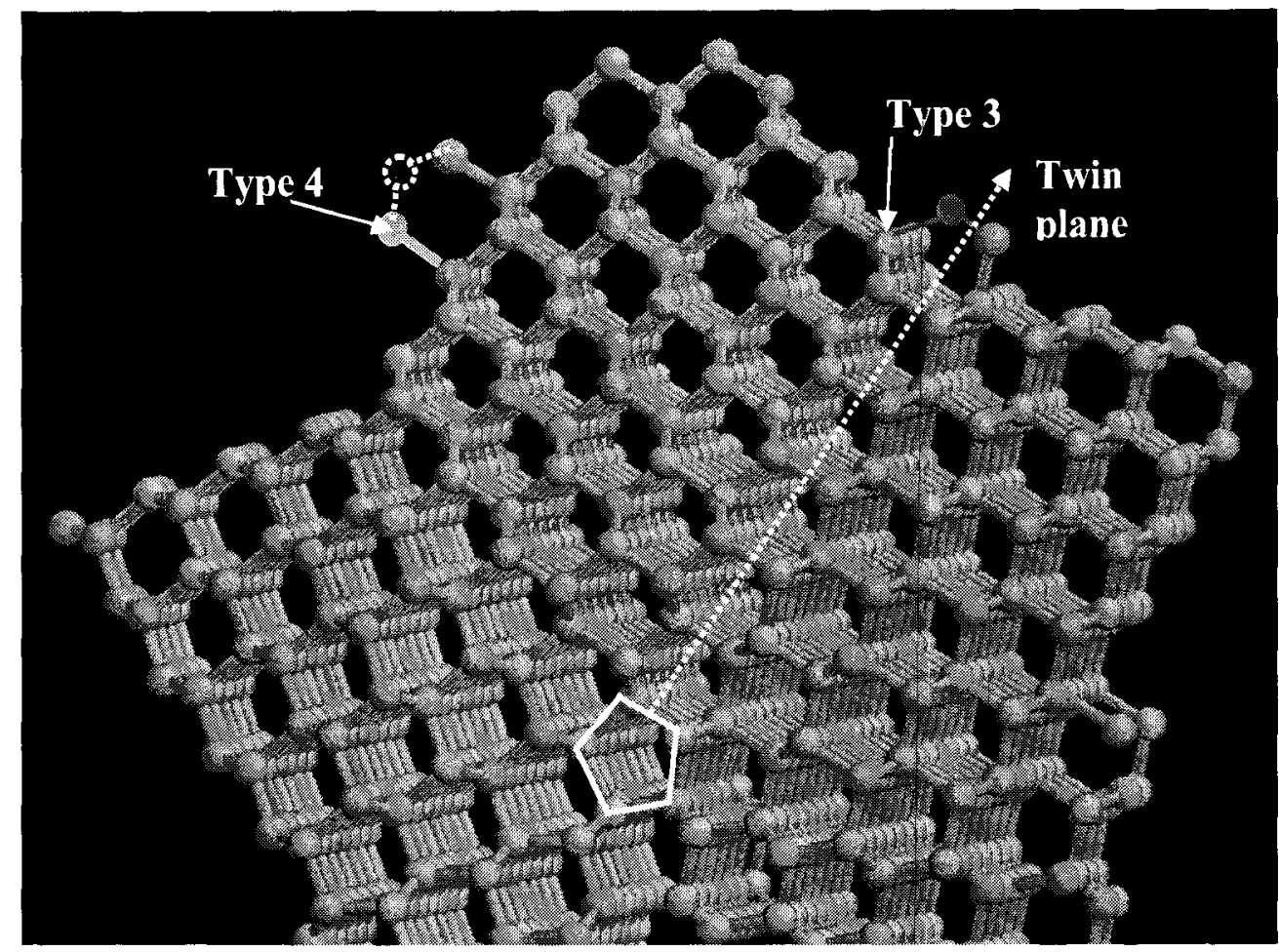

Figure 5.31 Ball and stick model indicating the two primary nucleation sites on a stardecahedral crystal: type- 3 at the twin boundary and type- 4 on a $\{111\}$ plane.

When the rate constant for adsorption at type 3 sites is increased (as in the case of decahedron), the tendency to nucleate at type 3 site increases due to the added advantage of needing only 2 atoms for a stable step. The simulations indicate that the dimples fill up and the additional $\{111\}$ facets along the twin boundaries fill up when high adsorption is assumed at these type of sites, giving a decahedral shape. In addition, the increase in kink nucleation makes the step spread out, giving it a shape of a triangle that is inverted with respect to a star-decahedral crystal. Another clear validation for this mechanism is seen in the simulations for icosahedral crystals. Twenty-fold crystals grown using the same rate constants as in star-decahedral resulted in significant dimples at the twin- 
centers, while the twenty-fold crystals with rate constants as in decahedral crystals did not have any dimples (Figure 5.26). Thus, the main reactions that control a stardecahedron vs. decahedron are 2,4 and 8 (Refer to table 5.1).

The previous set of simulations described in section 5.3.2.2 with etching of doubly bonded atoms also gave partially successful simulations for star-decahedral crystals. However, these star-decahedral crystals did not have the dimple at the center, and also they had some $\{100\}$ faceting and hence were called star-dodecahedral crystals. Doubly bonded atoms that were etched occurred on sites as indicated in Figure 5.32.

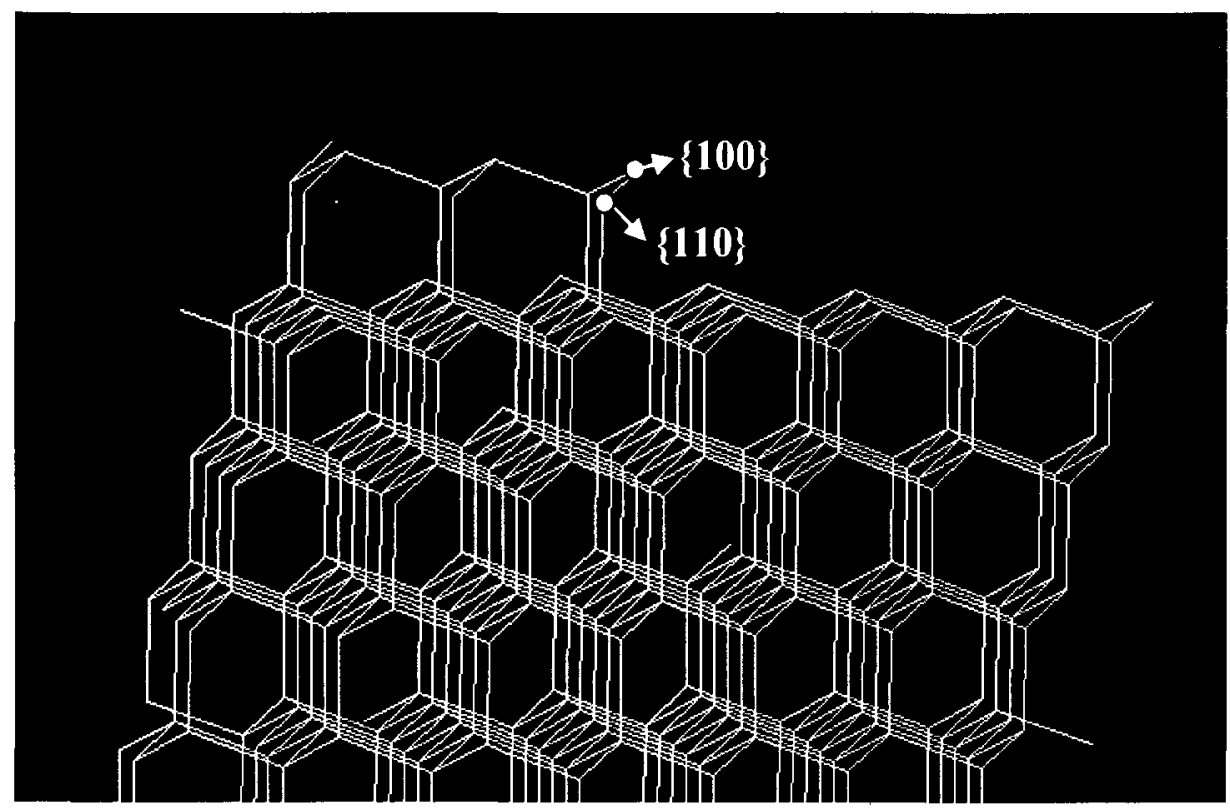

Figure 5.32 Wire-frame model indicating the types of doubly bonded atoms that would be etched in simulations where $\mu_{2} \neq 0$.

As indicated in Figure 5.32, the doubly bonded sites are of two types: $\{100\}$ sites and $\{110\}$ sites. Specifically etching of the $\{110\}$ type site, would give rise to a type 3 site. Hence, etching on these sites is one way of reducing the adsorption on type 3 site, 
which indirectly prevents the propagation of type 6 site. The etching probability was kept low (only $1 / 10$ to $1 / 100^{\text {th }}$ of adsorption probability). With such a low probability, the chances of choosing the $\{110\}$ type site would be low. This could be a reason for not observing a well-defined $\{111\}$ faceting along twin planes (i.e. the star-shape), and not observing dimples at all.

The important issue to be addressed is what would give rise to the slow adsorption on a type 3 site or type 6 sites in one case versus the other. Both these events require the adsorption of two successive adatoms to form a stable structure. It could thus be speculated that the high adsorption on site types 3 and 6 would be faster if a $\mathrm{C}_{2} \cdot$ type radical would adsorb at these sites, making them stable quickly, or in another way, the adsorption of two consecutive methyl species occurred in quick succession. It was shown earlier that acetylenic species $\left(\mathrm{C}_{2} \mathrm{H}_{2}\right)$ are more favorable for addition at the $<110>$ steps on the $\{111\}$ surface (type 3 , and type 6 ) by using quantum mechanical simulations [40] and methyl species are more favorable for $\{100\}$ type sites (type 2) [170]. Thus, it could be probable, that star-decahedral crystals grow in an environment that does not favors $\mathrm{C}_{2}$. adsorption while decahedral crystals grow in an environment that favors $\mathrm{C}_{2} \cdot$ adsorption. Further using dynamic Monte Carlo simulations, Frenklach [171] proposed that addition of $\mathrm{C}_{2} \mathrm{H}_{2}$ creates sites for $\mathrm{CH}_{3}$ adsorption and adsorption of $\mathrm{CH}_{3}$ creates sites for $\mathrm{C}_{2} \mathrm{H}_{2}$ adsorption. This is also seen in our simulations, in which a kink is nucleated by a two atom addition on type 6 site (similar to $\mathrm{C}_{2} \mathrm{H}_{2}$ ). Once this kink nucleated, it creates sites where a single methyl is required for adsorption (type 2 sites).

However, this does not necessarily imply that the gas phase composition in the growth conditions for decahedral or star-decahedral crystals would have to be different. 
In fact, it was shown that at low hydrocarbon/hydrogen ratios, the surface temperature alone determines the kinetics of gas-surface reactions $[79,80]$. Hence, what could be inferred is that in the case of star-decahedral and decahedral crystals, it is the temperature at the surface that determines which reaction is favored versus the other or which species is adsorbed faster than the other. Calculations of surface kinetics of these site-specific reactions could give further proof to this hypothesis.

\subsection{Summary}

Experimental and theoretical studies were performed to understand faceting of multiply twinned diamond crystals. In this work, the common experimentally observed twinned morphologies were star-decahedral crystals and dimpled-icosahedral crystals. These crystals had characteristic additional $\{111\}$ facets along twin boundaries and a dimple at the twin centers. For the first time, a close indication of the change in morphology of a star-decahedral to an icosahedral crystal was obtained experimentally.

The dimples and additional $\{111\}$ facets seemed to be due to specific kinetic events occurring during growth. KMC simulations were performed to understand which kinetic events decide the shape i.e. decahedral or star-decahedral. The conclusions from these simulations were that when a two-atom adsorption event for $\{111\}$ steppropagation becomes limiting (slower kink nucleation), the observed morphology is a star-decahedron. While, when the two atom addition event is fast (quick $\{111\}$ steppropagation and kink nucleation), the final morphology is a decahedral crystal. Low adsorption of $\mathrm{C}_{2}$ radicals to the surface could possibly give rise to slow two-atom addition events, leading to star-decahedral crystals. 


\section{CHAPTER 6}

\section{KINETIC FACETING OF CARBON NANOTUBES - CARBON NANOPIPTTES}

\subsection{Introduction}

Catalytic growth of carbon nanotubes and other nanostructures has been known since $1950[110,111]$. Carbon nanotubes are typically cylindrical, with single or multiwalls, whose catalyst-assisted growth mechanisms are well understood. On the other hand, the nucleation and growth of conical carbon nanostructures are difficult to explain. As discussed in Chapter 2, conical morphologies of carbon were reported in many random observations and still need to be understood in terms of both their structure and growth mechanisms. Also, these conical nanostructures are interesting because of their nanoscale tips, rigidity and different surface configurations from the carbon multi-walled nanotubes.

Hollow conical structures, with open ends, could have many interesting applications, such as fluid flow, in addition to possibly sensing applications due to their surface sites. However, these nanostructures have to be grown in controllable morphologies and in arrays, to utilize them in specific applications.

This chapter describes the discovery, synthesis and structure of a tapered morphological manifestation of carbon nanotubes termed here as "carbon nanopipettes". In the experiments on nucleation of diamond using platinum wires immersed in the 
plasma (Ch. 4), it was expected that the temperatures and concentration of gas phase radical and neutral species would vary along the length of the platinum wire [143], and thus a variety of carbon phases were expected. Carbon nanopipettes nucleated along with diamond crystals on the platinum wires. Due to their tapered morphology, very different from any of the reported cylindrical or conical carbon nanostructures, their growth mechanisms were studied to develop a method for producing them as arrays.

\subsection{Synthesis of carbon nanopipettes}

In the same experiments for growing nanocrystalline carbon phases, as the platinum wire was placed vertically in the plasma, there was a variation in the type of deposit found along the wire length due to the variation in temperature and gas phase composition along the length of the substrate. The deposit at the tip of the platinum wires contained nanocrystalline graphite, diamond and other metastable phases of carbon like fcc-carbon, bcc-carbon and lonsdaleite. In regions away from the tip, the platinum wire was coated with microcrystalline diamond (crystal sizes $\sim 10 \mu \mathrm{m}$ ). In this region along with diamond crystals, several whiskers of about $1 \mu \mathrm{m}$ in diameter at the base and $20 \mu \mathrm{m}$ in length were observed. These whiskers appeared to emerge from the microcrystalline diamond film. It must be pointed out that the gas phase composition of $1-2 \% \mathrm{CH}_{4} / \mathrm{H}_{2}$ used in the synthesis of this work was atypical for carbon nanotube growth (which typically occurs with $20-30 \% \mathrm{CH}_{4} / \mathrm{H}_{2}$ ).

These whiskers had an outer conical morphology with a pointed tip, while their base was in the sub-micron regime (Figure 6.1). They were specifically called carbon nanopipettes for the reasons that will be stated in further discussions. The SEM-EDS 
taken from these whiskers indicated the presence of carbon. However, this could have been due to the heavy signal from the underlying diamond film and hence was not too reliable.

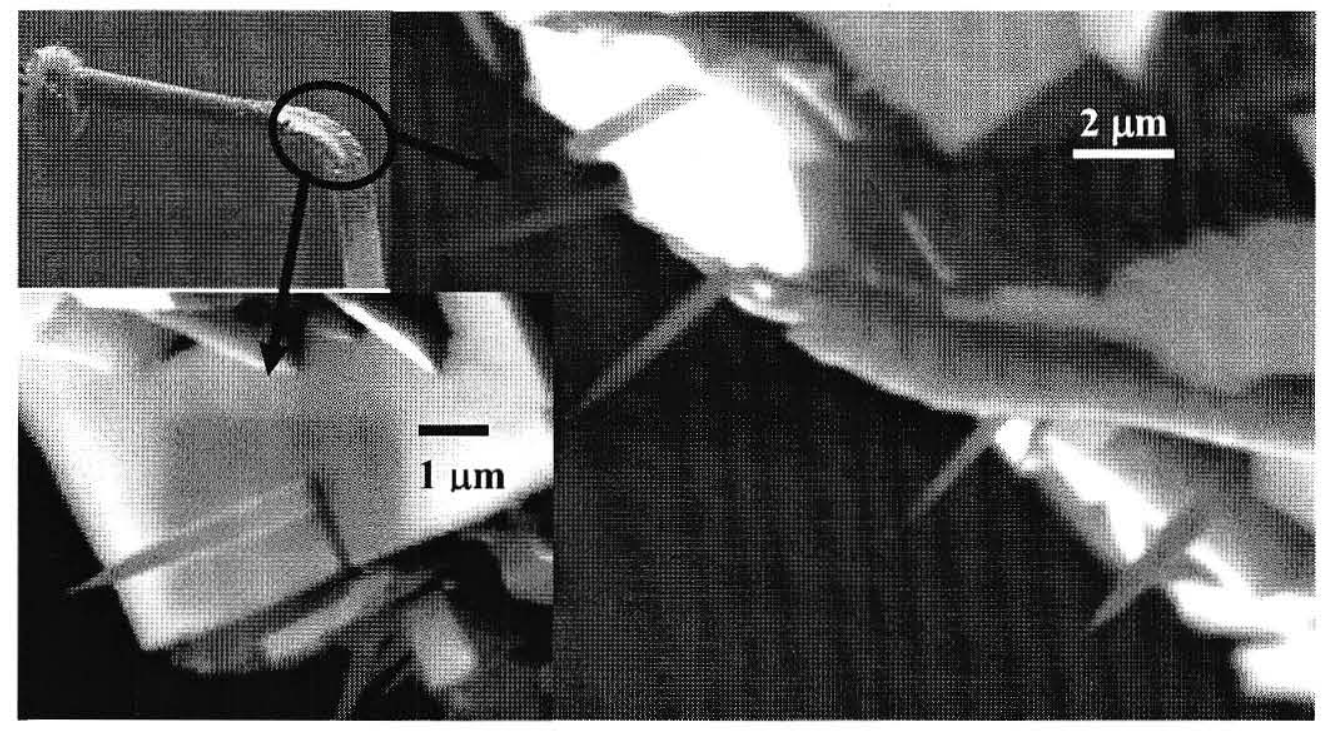

Figure 6.1 SEM image of carbon nanopipettes emerging from the microcrystalline diamond film on a platinum wire 


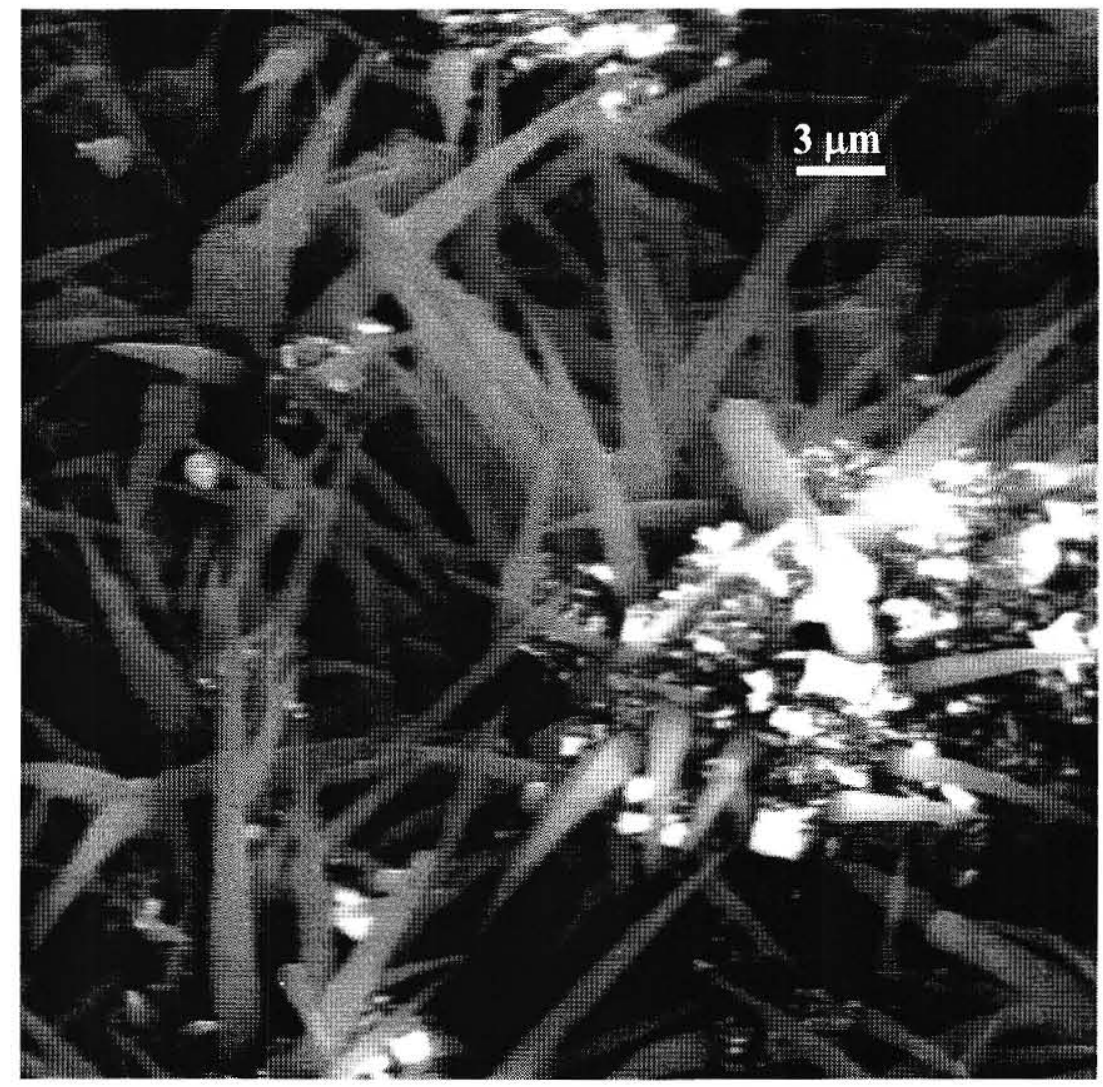

Figure 6.2 SEM image showing a large density of randomly oriented carbon nanopipettes.

Higher resolution SEM on these whiskers showed surface faceting as indicated in Figure 6.3. They seemed to be made up of some rolled up sheets. Figure 6.3 (b) shows a partially unwrapped nanopipette that could have been caused by beam damage. 


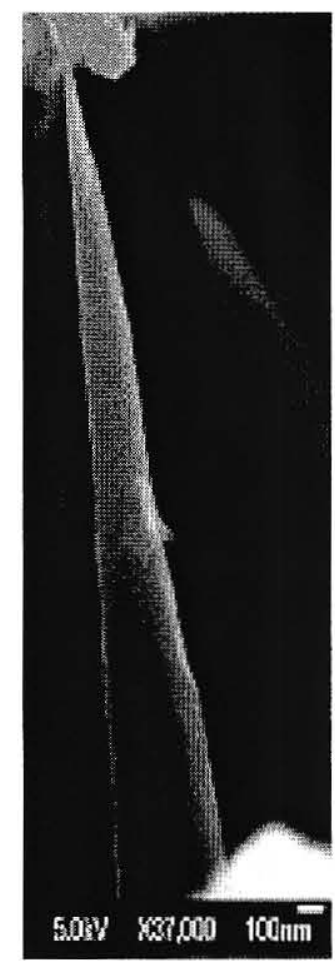

(a)

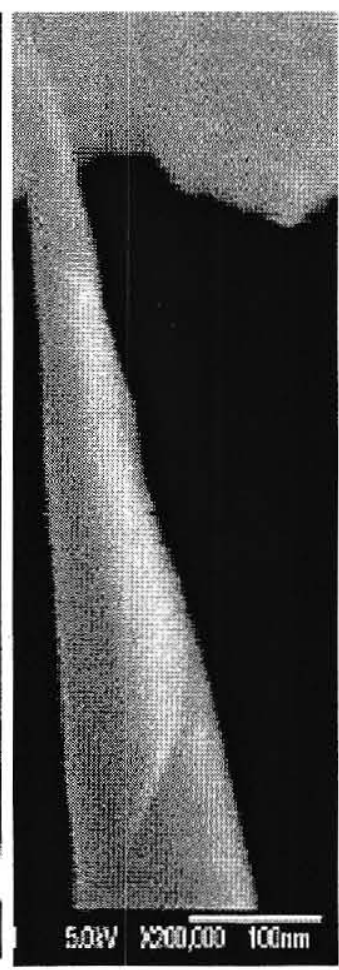

(b)

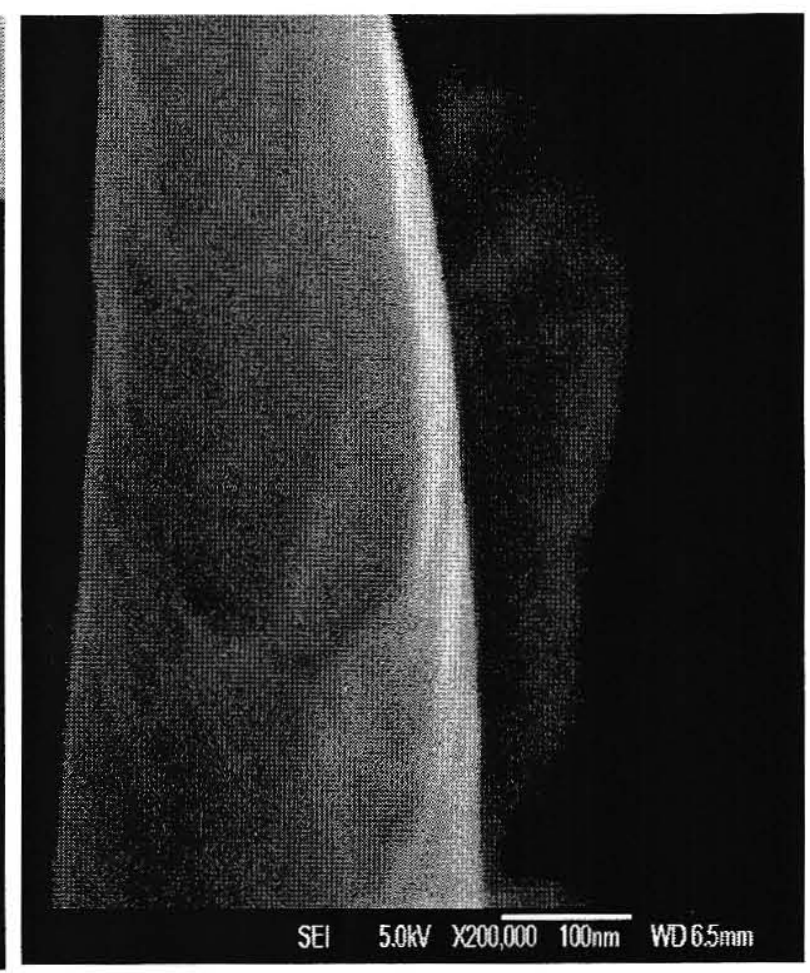

(c)

Figure 6.3 (a-c) SEM images indicating surface faceting of the nanopipettes, and appearance of rolled-up sheets.

\subsection{Structural characterization}

Micro-Raman spectra taken on these nanopipettes emerging from the diamond film is shown in Figure 6.4. The spectrum indicated as ' $a$ ' is taken from the microcrystalline diamond adjacent to the nanopipettes, and the spectrum indicated as ' $b$ ' is taken from the individual nanopipettes. As shown in the figure, the microcrystalline diamond shows peaks corresponding to 1242 and $1325 \mathrm{~cm}^{-1} .1332 \mathrm{~cm}^{-1}$ is the characteristic signature for pure diamond $\mathrm{sp}^{3}$ phase. Systematic Raman studies of borondoped diamond thin films have indicated that the $1332 \mathrm{~cm}^{-1}$ line becomes asymmetrical at higher boron concentrations due to the Fano-interference between the zone center Raman 
active phonon and an electronic continuum induced by the impurity band [172]. In addition, a new broad Raman peak is activated around $1200 \mathrm{~cm}^{-1}$ [172]. Hence, the asymmetric peak at $1325 \mathrm{~cm}^{-1}$ and $1240 \mathrm{~cm}^{-1}$ are assigned to boron doped microcrystalline diamond.

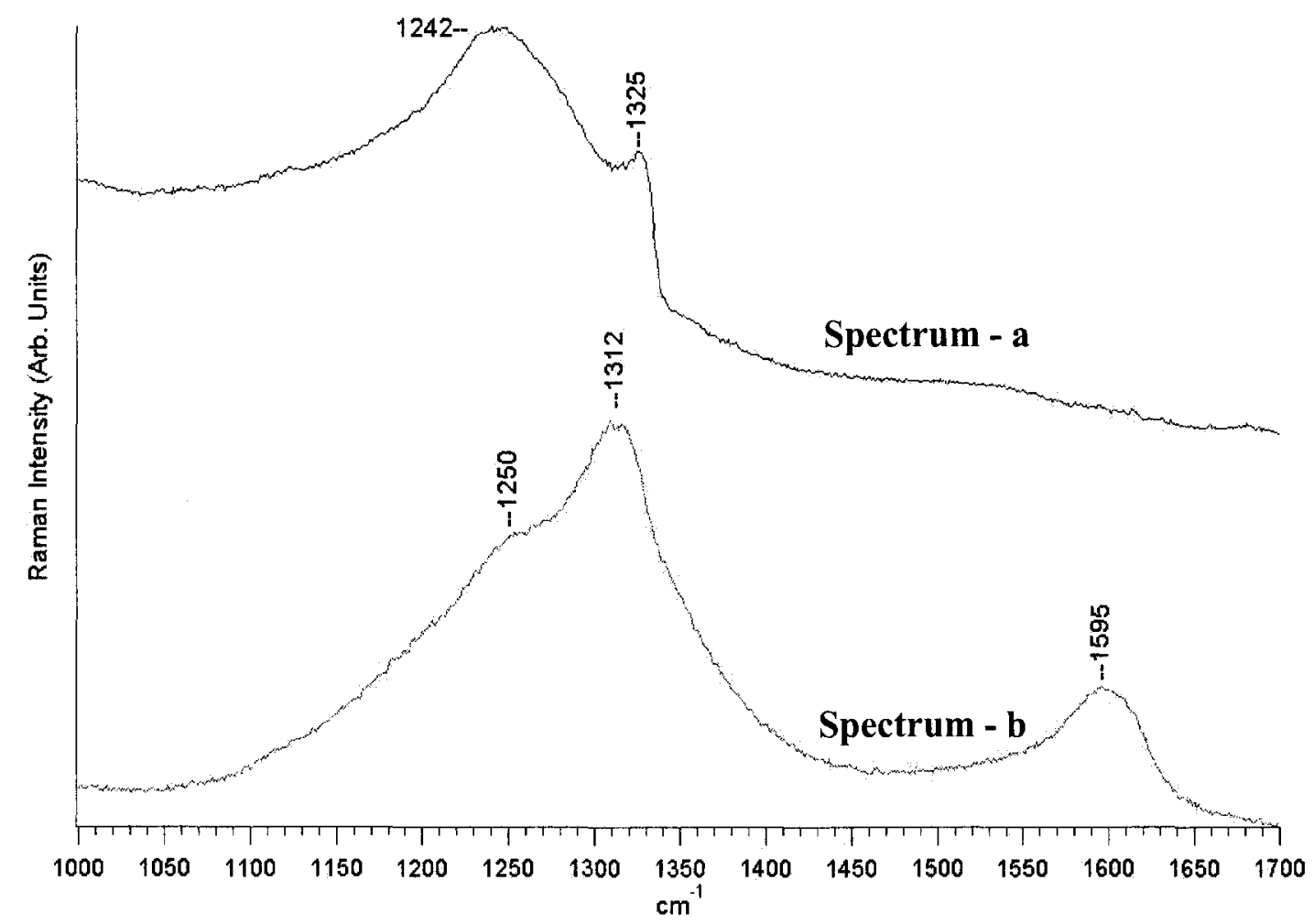

Figure 6.4 Raman spectra taken with laser wavelength $785 \mathrm{~nm}$ from (a) microcrystalline diamond film and (b) nanopipettes emerging from the diamond film

The carbon nanopipettes show peaks at $1312 \mathrm{~cm}^{-1}$ and $1595 \mathrm{~cm}^{-1}$, which are attributable to the D-and G-band of graphite for the particular wavelength of the laser used in the Raman spectra [173-175]. There appears a small shoulder at $1250 \mathrm{~cm}^{-1}$, which could be attributed to the underlying diamond film. Thus, Raman analysis indicated that the carbon nanopipettes could actually be graphitic in nature. 
Transmission Electron Microscopy (TEM) was used for detailed structural investigation. Few carbon nanopipettes were scraped from the platinum substrate and put on a TEM grid. These pipettes were analyzed in a JEOL model 2010 TEM. As seen in the bright field TEM images (Figure 6.5), the pipettes are several micrometers long with a well-defined uniform-diameter hollow core. The core is approximately $10-20 \mathrm{~nm}$ in diameter, extending throughout the length of the pipette.

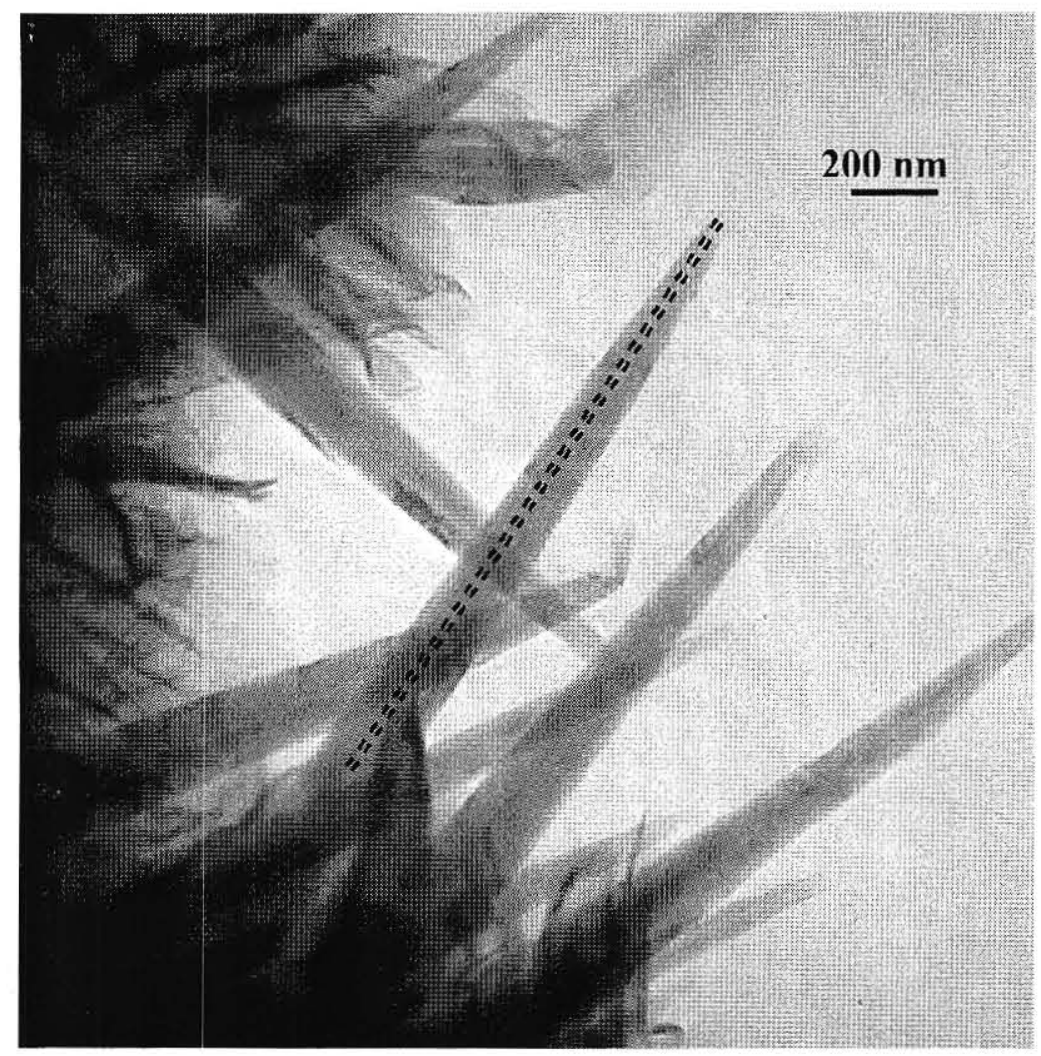

Figure 6.5 Bright field TEM image of the nanopipettes, indicating a hollow core (highlighted, running axially down as indicated).

The dark field image in Figure 6.6 highlights this structure even further showing the hollow core running across the entire length of a pipette. At the tip of the pipette, 
where the thickness permitted a reasonable signal, an energy-filtered image using the $\mathrm{sp}^{2}$ core loss peak clearly illuminated the specimen (Figure 6.6a, inset). The dark region running down the axis of the pipette corresponds to the hollow core, which evidently does not contribute to any signal (in this case inelastically scattered core loss electrons). Based on the energy loss images, the walls of the pipette at least in the tip region appeared to be graphitic in nature.

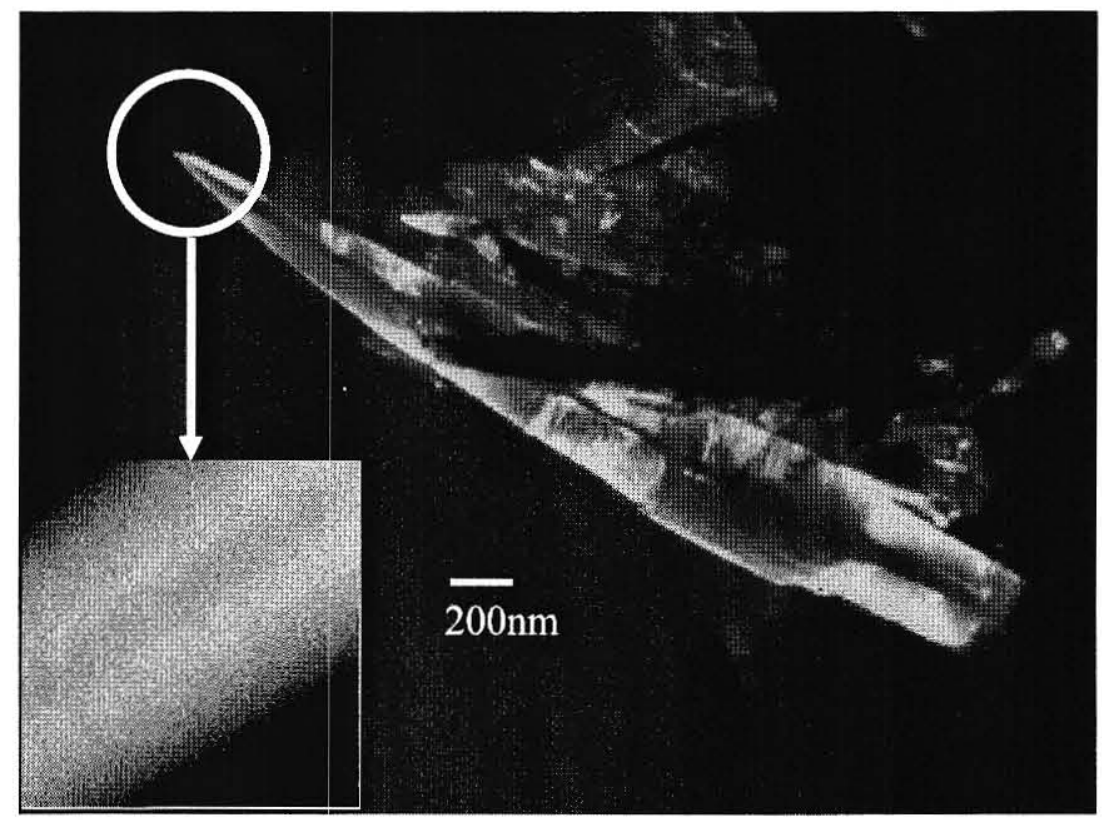

Figure 6.6 Dark field TEM image taken from the 00.4 Bragg reflection, and inset indicating the corresponding EFTEM image from the tip of the nanopipette using $\mathrm{sp}^{2}$ carbon core loss peak

The graphitic structure of the nanopipettes was confirmed by the basal plane lattice images. Electron diffraction patterns from thicker regions of the pipette (Figure 6.7) exhibited characteristic features of helical sheets of graphite. The pitch angle associated with this type of structure varied at different points (an angle of $9^{\circ}$ is indexed 
in Figure 6.7b) indicating a more complex morphology. TEM/EDS did not detect the presence of platinum throughout the length of the pipettes.
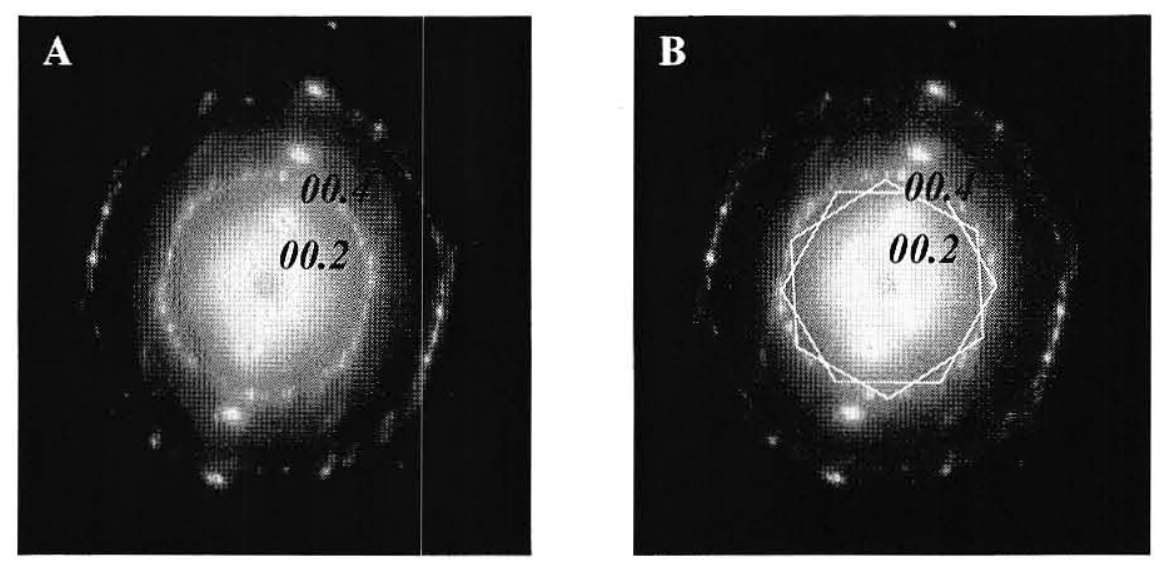

Figure 6.7 (a) Diffraction patterns from carbon nanopipettes indexed as basal plane of graphite. (b) Diffraction pattern outlining the helical sheets with angle $\sim 9^{\circ}$.

Figure 6.8(a) shows the dark field image produced by the 00.4 Bragg reflection of a broken pipette. This image clearly shows a central tube emerging from the thick pipette. The outer wall of this central tube seems to have the same brightness as the shell structure of the pipette, indicating that it has a similar crystal structure as the shell. The diffraction pattern taken at an electron transparent region of the shell in 6.8(b) is similar to that shown in 6.7 , once again indicating that this structure is made up of helical sheets of graphite. 


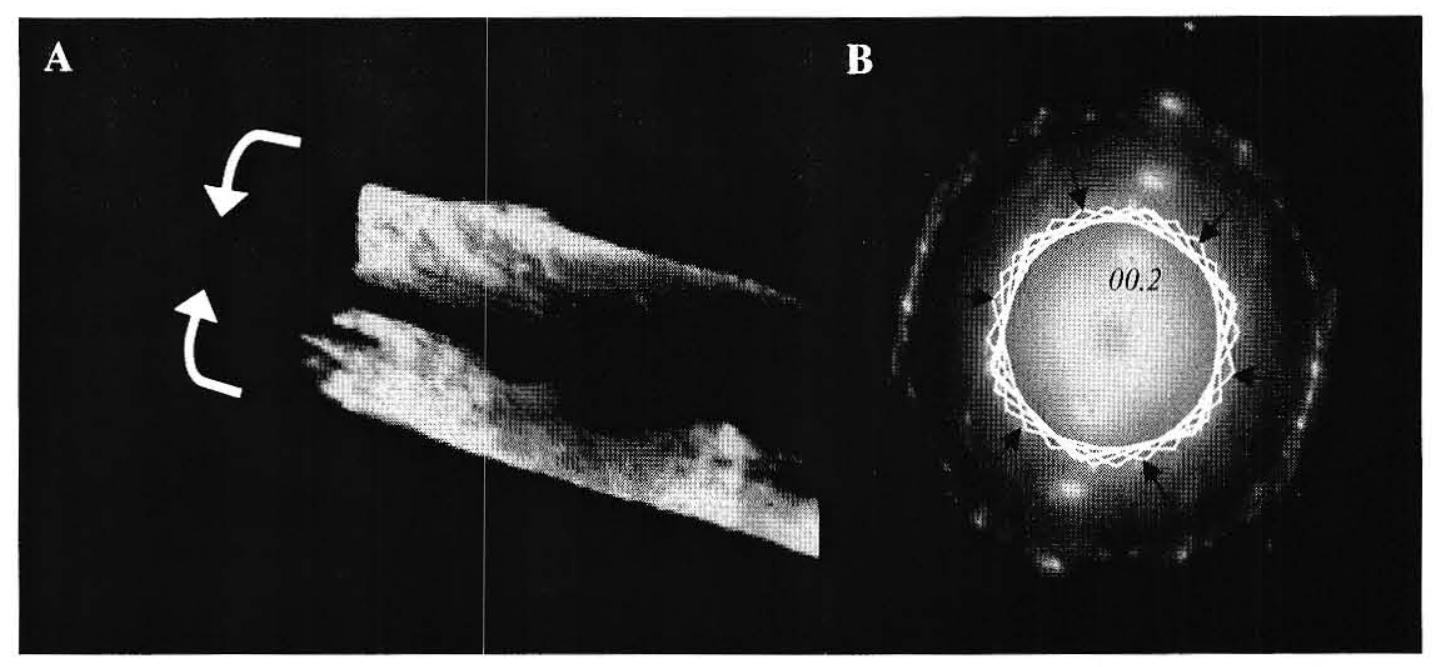

Figure 6.8 (a) Dark field image of a broken nanopipette, indicating a protruding central nanotube, which has the same brightness as the shell of the pipette. Diffraction pattern from the broken nanopipette corresponding to helical sheet of graphite.

TEM investigations suggested that these "nanopipettes" are hollow whiskers made up of helical sheets of graphite. They have a core of constant diameter of 10-20 nm running throughout their length. These nanopipettes are open at both ends because the core extends throughout their length. Their outer morphology however is conical, with a base of $1 \mu \mathrm{m}$ and a tip equal to that of the hollow core, i.e. $10-20 \mathrm{~nm}$. Their outer shell is made up of helical sheets of graphite. The overall morphology is shown diagrammatically in Figure 6.9. So, in order to understand the growth mechanism of these nanopipettes, experiments to study their early stages of growth were carried out. 
Figure 6.9 Schematic of the overall structure of the carbon nanopipette.

\subsection{Growth mechanism of nanopipettes: early stages of growth}

As discussed in Chapter 2, conical structures of graphite had been studied long before the discovery of carbon nanotubes. Some of the earliest research on onedimensional nanostructures of graphite described a model for the growth of graphite whiskers [110]. According to one of the models, a sheet of graphite (consisting of several monolayers) overlaps itself and continues to wind around the whisker axis many times rather than joining opposite edges and forming concentric tubes. This scroll structure gave rise to a simultaneous thickening and growth along the length. However, this morphology was different from the one shown in Figure 6.9. In another attempt, while heating silicon carbide crystals, graphite whiskers were obtained [120]. The explanation for columnar growth was given on the basis of classical model of whisker growth initiated by nucleation of a dislocation with a screw component perpendicular to the surface. A time dependent impurity adsorption hampers layer spreading. Another explanation for these needle-shaped crystals was that a flat graphite sheet rolled into a cone, and each time it wraps around by lifting itself to form a rotation over a small angle. 
Graphite sheets are known to grow along their edges, thus if subsequent growth takes place, a helical surface will be formed by growth along steps (to form a spiral shape) [176].

Graphite polyhedral crystals, synthesized from gas phase, had folded and closed graphene planes different from ordinary graphite but similar to multi-walled carbon nanotubes [124]. There were small angle conical crystals also [125], which were as large as $3 \mu \mathrm{m}$ and $300 \mathrm{~nm}$ in diameter. Some of these crystals were hollow, consisting of straight and well-defined $110 \mathrm{~nm}$ thick walls. Their hollow tips had a herringbone type structure, however beyond the tip, this herring bone structure transformed into a conical cavity. None of the above conical crystals had a structure similar to the carbon nanopipettes described in the previous section. Hence, none of the above mechanisms could explain the formation of a whisker that is conical on the outside and has a uniform diameter hollow core at the center.

Some studies on the spatial profiles of microwave plasmas have indicated that the gas temperatures near the center of the microwave plasma could reach as high as $2000^{\circ} \mathrm{K}$ [81]. Also, it was experimentally determined that with the change in one $\mathrm{cm}$ length along the radius of the plasma, the temperature reduced by about $1000 \mathrm{~K}[82]$. Since the substrate is placed vertically, the plasma tends to discharge at the tip, making it close to the melting point of Platinum $\left(1768^{\circ} \mathrm{C}\right)$. The pipettes seemed to be growing a few $\mathrm{mm}$ below the tip of the wire, which could be estimated to be about $500 \mathrm{~K}$ lesser than the tip. These high temperatures and the peculiar conical shape suggest the possibility that the growth mode could be due to an evaporating catalyst i.e., a platinum catalyst particle initiates the growth of multi-walled carbon nanotubes. As the pipette grows, the catalyst 
droplet evaporates. As the catalyst droplet evaporates, the outer diameter of the multiwalled shell reduces. This hypothesis could be easily tested using a sequential step experiment by growing for shorter time scales and seeing the catalyst at the tip.

Hence, in another experiment, platinum thin films $(\sim 50 \mathrm{~nm})$ were electrodeposited on platinum wires coated with microcrystalline diamond films. Diamond films acted as a non-reactive stable substrate. These Pt-diamond-Pt wire substrates were immersed into the plasma, but inclined at an angle to the substrate. The platinum coating on the diamond film melted into droplets when exposed to the plasma, due to temperature gradients. The growth experiments were performed for over a period of one hour or less. As shown in Figure 6.10 , there is a continuous variation of the morphology of the one-dimensional structures along the wire substrate. In a region close to the tip of the substrate, conical structures with a multi-walled (or single-walled) carbon nanotube emerging from the center are observed (Figure 6.10a). The nanotube is surrounded by graphite deposit. When the distance from the tip increases, there is a competition between the etching and growth of crystalline phase $\left(\mathrm{sp}^{2}\right)$ of carbon. Due to this, the central nanotube remains intact, while the surrounding graphite material tries to grow rapidly around it. However, hydrogen plasma tends to etch the graphitic deposit, and etching seems more prevalent near the tip of these nanopipettes, giving a conical structure. The relative degree of growth and etching varies with the position in the plasma. Thus, the aspect ratio of the structures increased with increasing distance from the tip of the substrate, ultimately giving rise to nanopipettes further distance away (Figure 6.10c)). The density of the pipettes also reduced along the length of the substrate. 


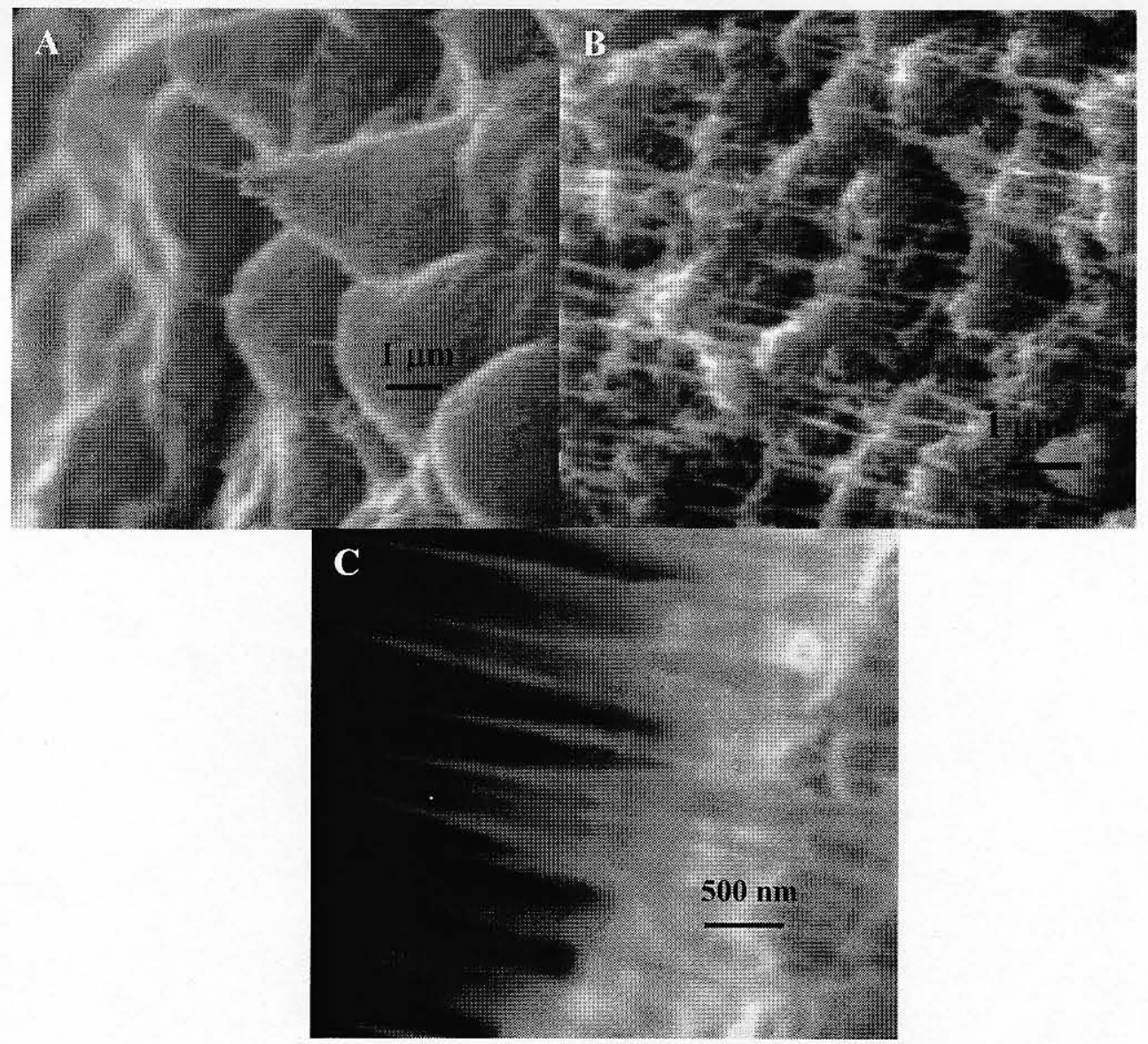

Figure 6.10 Varying aspect ratio structures along the length of the platinum wire: (a) conical structures with a central nanotube, (b) increased aspect ratio of conical structures, and (c) carbon nanopipettes.

If the model of an evaporating catalyst were true, then such a variation of morphology of one-dimensional structures along the substrate would not be observed. The morphology seen in Figure 6.10 would mean that the rate of evaporation changes abruptly during the growth of the pipette, which would be quite impossible. Hence, these experiments clearly indicate that selective etching and growth simultaneously is 
responsible for the growth of these conical nanopipettes with different aspect ratios along the length of the substrate.

Figure $6.11(a-b)$ shows a schematic of the growth mechanism of the carbon nanopipettes. Overall, there is an initial open-ended nanotube that grows out rapidly due to the presence of platinum metal catalyst. The high temperatures in the plasma eventually evaporate the catalyst particle, due to which, Pt is never observed in the final morphology. Surrounding this nanotube, helical sheets of graphite coil around each other. The continuous coiling around of graphite sheets makes the outer morphology of the nanopipette conical, while the initial nanotube maintains the inner constant-diameter hollow core. Depending on the placement in the plasma, there is competitive growth and etching of the graphite sheets surrounding the central nanotube. On the part of the substrate that is immersed into the plasma, etching as well as growth seems to dominate, because of the high concentrations of gas phase radicals present at this region, giving rise to a low aspect ratio. Away from the tip region of the substrate immersed in the plasma, growth seems to dominate giving rise to high aspect ratio pipettes. The inner core diameter is however larger than a SWNT (which is typically 1-4 nm), the reason being that it has surrounding graphite shells. Similar to a MWNT, the nanopipette is able to support larger wall diameter. The low aspect ratio pipettes grow towards the tip of the substrate exposed to the plasma, and the high aspect ratio ones grow at the regions away from the tip of the substrates. 


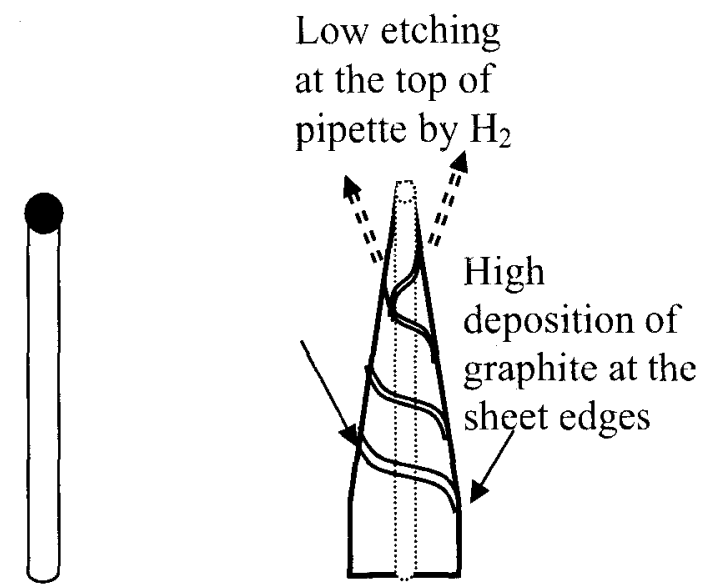

Low aspect ratio

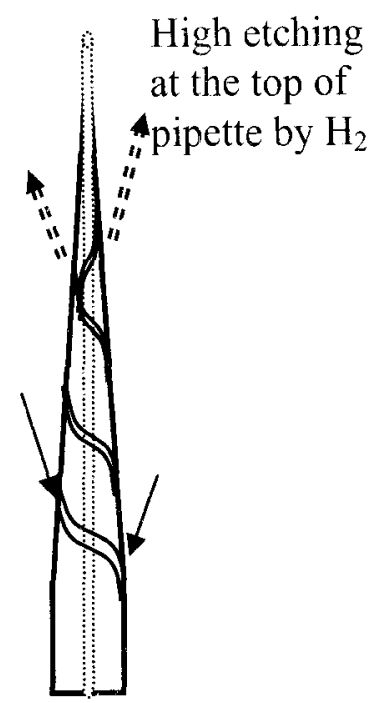

High aspect ratio (a) Growth begins with a nanotube catalyzed by $\mathrm{Pt}$ (b) Wrapping up of helical sheets of graphite around the central nanotube disappearance of catalyst droplet

Figure 6.11 Growth mechanism of carbon nanopipettes: (a) growth begins with an initial nanotube, (b) wrapping-up of helical sheets of graphite around the central nanotube (c) simultaneous growth and etching results in high aspect ratio pipettes.

The particular external faceting of carbon nanopipettes makes their surface surrounded by the highly reactive edge planes of graphite, contrary to carbon nanotubes, which have almost inert basal planes. This could make them attractive for applications that could make use of their surface reactivity such as chemical/electrochemical sensing (discussed in more detail in chapter 7).

The length of the pipettes can be controlled by increasing the growth time. As shown in Fig. 6.12, pipettes as tall as $75 \mu \mathrm{m}$ were grown. However, arrays of pipettes of this length have not yet been obtained. Also, shown in Figure 6.13 is an array of 
nanopipettes grown on a sheet of platinum, placed in the plasma in the same manner as the platinum wires. A point to be noted is that the pipettes grow at the edges of the sheet (the thickness of the sheet substrate). This could be due to the fact that the plasma tends to discharge more on the edges of a substrate, creating higher temperatures in those regions, close to the melting point of the platinum rather than the top flat surface. An array grown on a flat sheet would be easier to handle, in terms of pealing the pipette array out of the substrate.

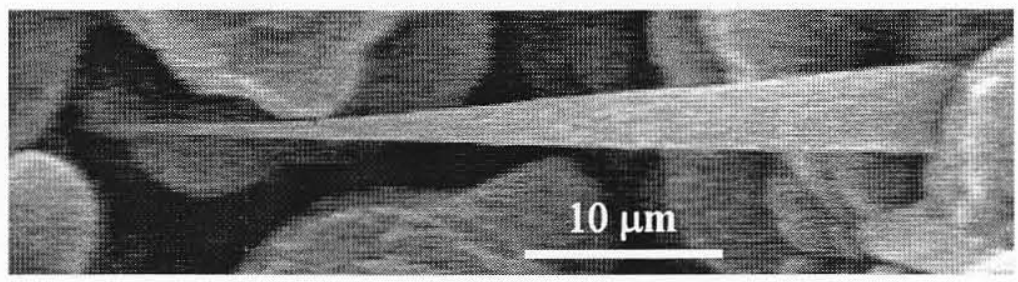

Figure 6.12 SEM image of a $75 \mu \mathrm{m}$ long carbon nanopipette.

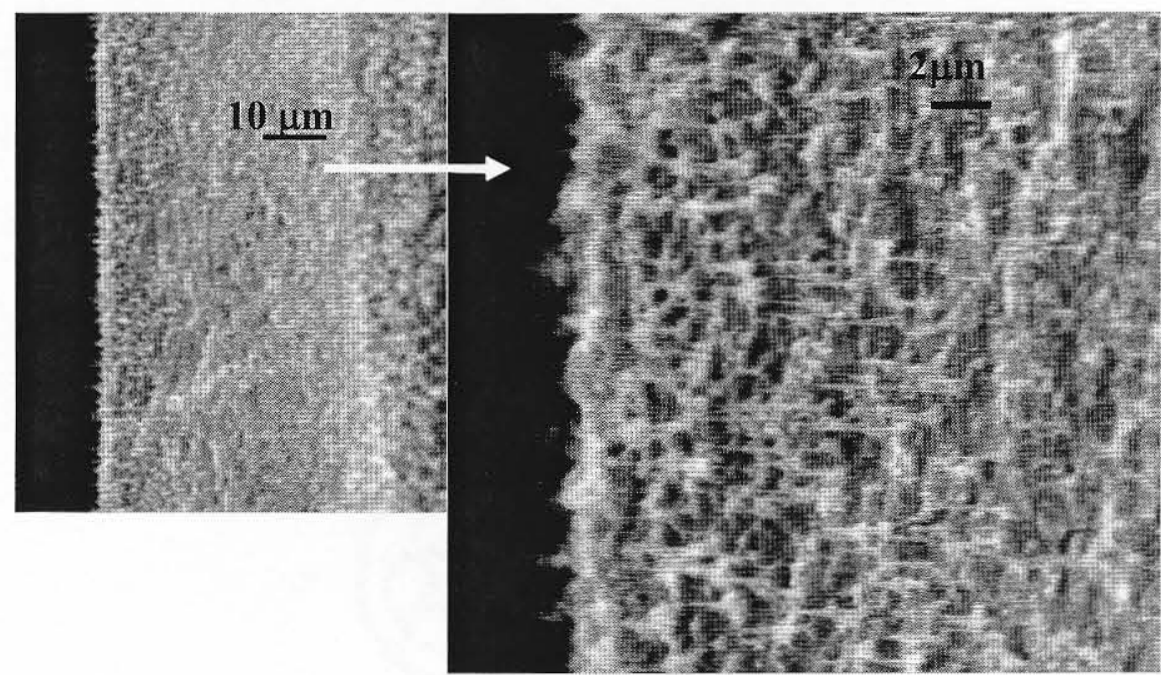

Figure 6.13 SEM image of carbon nanopipette array on a platinum sheet substrate. 


\subsection{Nanostructures grown on gold substrates}

When gold wires were immersed into the microwave plasma (in the same manner as platinum) at the same gas phase conditions of $1-2 \% \mathrm{CH}_{4} / \mathrm{H}_{2}$, one dimensional structures that were similar to nanotubes, with constant outer wall diameters of about 80 $90 \mathrm{~nm}$ were obtained. In some regions, a graphite deposit appeared to be growing around the central nanotube (See Figure 6.14).

Structural investigations using TEM indicated that they are multi-walled carbon nanotubes, although the actual diffraction patterns of these samples were not recorded. Also, no other experiments were carried out on gold to investigate these structures further. A possible explanation could be that the catalyst used (gold here, instead of platinum) could affect the morphology of the nanostructures, however, this would require further experiments to understand this phenomena. 

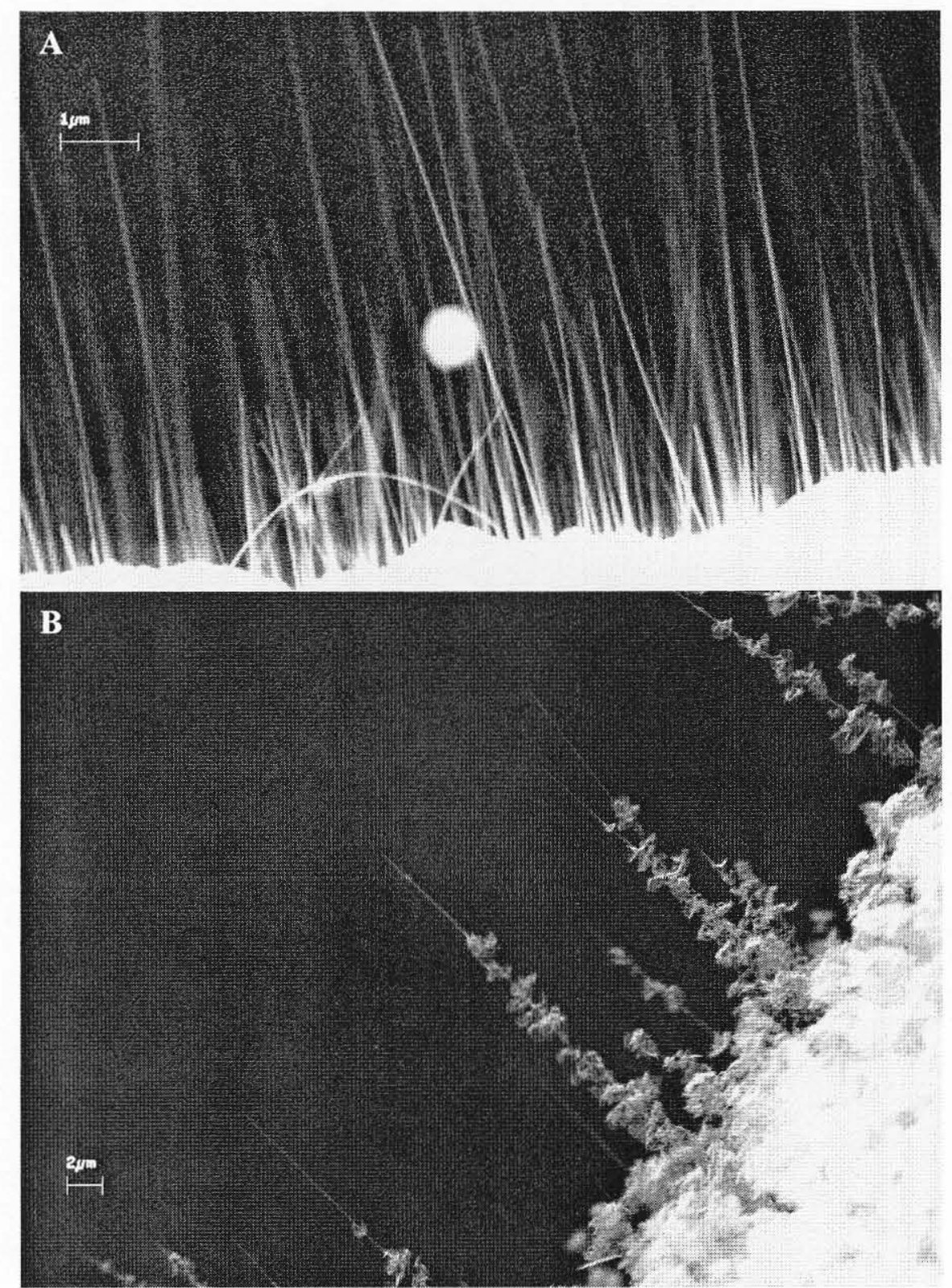

Figure 6.14 SEM images of (a) carbon nanostructures grown on gold wire-substrate and

(b) graphite deposit growing around the nanostructures. 


\subsection{Summary}

The synthesis, structural characterization and growth mechanisms of a unique morphology of carbon nanotube, called carbon nanopipettes was discussed in this chapter. Carbon nanopipettes have an internal hollow core $(10-20 \mathrm{~nm})$ and an outer conical shell due to helical sheets of graphite surrounding the internal core. Their peculiar structure arises due to the simultaneous growth and etching of graphite in the methane-hydrogen plasma. They can be grown in the form of arrays, aligned perfectly vertical to the substrate. Due to their peculiar structure, their surfaces can be expected to be made up of highly reactive edge sites making them an excellent candidate for electrochemical sensing. Their sharp tips would make them useful for piercing through a barrier, such as for transdermal drug delivery and for several electronic applications like AFM tips, field emission, etc. The additional property of a hollow core could make nanopipettes useful for fluid flow applications. 


\section{CHAPTER 7}

\section{ELECTROANALYTICAL SURFACE CHEMISTRY ON NANOCRYSTALLINE GRAPHITE MORPHOLOGIES}

\subsection{Introduction}

Nanocrystals of graphite, including new 1-D morphologies like carbon nanopipettes, are expected to have high edge-site density due to their small crystal domains [29]. Surface-site characterization is quite difficult for nanomaterials compared to single crystal surfaces. Fortunately, graphite is an anisotropic material with highly reactive edge-plane sites and nearly inert basal-plane sites. So, the electron-transfer kinetics at edge sites is expected to be significantly different from the basal plane sites. Thus, electrochemical techniques could allow one to characterize the surface sites of different forms of nanocrystalline graphite.

Carbon is one of the most promising electrode materials for sensing and fuel cell applications due to its inertness and ease of polarizability. So, there is an ongoing interest to improve carbon based materials for electrode applications by using different phases at different length scales. Due to its biocompatibility, carbon has a great potential for electrochemical detection of biological solutes for both in vivo and in vitro environments. The neurological solutes of interest, such as dopamine, are mainly 
catechol and quinone-type compounds. The electron transfer reactions of these compounds are very sensitive to the type of carbon surfaces. Thus, dopamine has been used as a model compound for characterizing electrode surfaces.

Graphite is one of the first forms of carbon investigated as electrodes. Spectroscopic graphite, carbon paste electrodes (CPE), and Pyrolytic graphite (PG) were some of the well-studied forms of graphite electrodes [177-179]. Later, glassy carbon (GC) was introduced as an electrode material [180].

More recently, the electrochemical behavior of boron-doped microcrystalline diamond (MCD) has been extensively explored due to its many desirable properties including high chemical and electrochemical inertness, low background current and capacitance, wide working potential window for most solvents and electrolytes, and reproducible electrochemical activity without any pre-treatment [181-185]. All of these characteristics were a clear advantage over the different forms of bulk graphite and GC. The low and stable background current and capacitance characteristics of MCD have been attributed to factors such as a relative absence of electroactive carbon-oxygen functionalities on the hydrogen-terminated diamond surface and a lower density of surface electronic states near the Fermi level due to the boron doping [181]. However, due to the lack of carbon-oxygen adsorption sites [186], microcrystalline diamond films exhibit a high degree of irreversibility for redox reactions such as those of catechols and quinones. Nanocrystalline diamond has also been explored as an electrode due to the possibility of having different electrochemical characteristics on account of its higher surface/volume ratio and the specific nature of its surface sites [187-189]. However, the 
electrochemical behavior of nanocrystalline diamond was also shown to be sluggish for adsorption-related electron transfer reactions with catechols and quinones [187].

In comparison to any other material, $\mathrm{GC}$ has still been attractive for the analysis of catechol and quinone compounds (neurological fluids). It has been shown that the surface preparation of GC is crucial to its electrochemical performance. In particular, the surface preparation affects the observed electron transfer kinetics, but optimum surface treatment techniques vary widely [190]. Furthermore, the prepared GC surface may also be short-lived necessitating frequent reactivation, which subsequently brings degradation to its performance.

Graphite, on the other hand, could serve as an attractive electrode material provided one could make predominant use of the edge planes over the basal planes. Nanocrystalline graphite (NCG) offers such a possibility. Though there have been a few attempts to obtain NCG [116-119], no information exists regarding the electrochemical behavior of graphite crystals at the nanoscale.

Other than the above discussed materials as bulk electrode, micro [191] and nanoelectrodes [192] are necessary for probing smaller areas, and for several other advantages such as fast steady state time scales, high currents for the same electrode area, small capacitance currents. Nano/micro electrodes have increased mass transport rate because of non-linear diffusion, which leads to an increase in current density and causes the electrode process to reach steady state very quickly. Thus, microelectrodes are useful for fast cyclic voltammetry and detection of intermediate, short-lived compounds, not achievable by macro electrodes. With the same view in mind, carbon nanotubes have recently been studied for their electrochemical properties [193-198]. In the case of 
carbon nanotubes, it has been shown that the pentagons at the tip of the nanotube, pentagon and heptagon defect pairs in the lattice of the nanotube are responsible for the oxygen reduction at their surfaces [193]. In fact, one would have to rely on the defect states within the graphene sheets for exterior parts of the nanotubes to be active toward significant electron transfer kinetics. Using one-dimensional carbon nanostructures having predominantly graphite edge-sites, such as carbon nanopipettes, may be very useful as nanoelectrodes in comparison to basal-plane dominated carbon nanotubes.

This chapter begins with the detailed structural characterization of nanocrystalline graphite films. The study of the type of surface sites at nanocrystalline graphite and carbon nanopipettes (graphitic whiskers) using surface electroanalytical techniques is presented next using dopamine as the model compound. Based on the type of their surface sites, the applications of nanocrystalline graphite films and carbon nanopipettes for electroanalytical sensing of important biological fluids are presented.

\subsection{Nanocrystalline graphite films: synthesis and characterization}

In the experiments to grow high density of carbon nanocrystals, a dense carbon deposit was obtained at the tip of the platinum wires immersed into a microwave plasma. The analysis of the crushed part of this deposit for identifying the structures of carbon nuclei was discussed in Chapter 4. Without destroying this bulb-shaped deposit, at the tip of the platinum wire, several characterizations was performed like scanning electron microscopy-electron dispersive spectroscopy (SEM-EDS), X-ray Diffraction (XRD), Raman spectroscopy, and scanning tunneling electron microscopy (STEM). Each of these will be described here in detail. 
At the end of 24 hours of deposition, the tip of the platinum wire immersed vertically into the plasma had a black-colored, ball-shaped deposit. The SEM image and corresponding EDS is shown in Figure 7.1. As indicated in the figure, the ball-shaped tip is covered by a deposit that is composed of carbon only. The silicon signal seen in Figure $7.1 \mathrm{~b}$ is due to the underlying substrate holder on which the sample was placed for EDS. No platinum was detected in this deposit within the detection limit of the EDS.

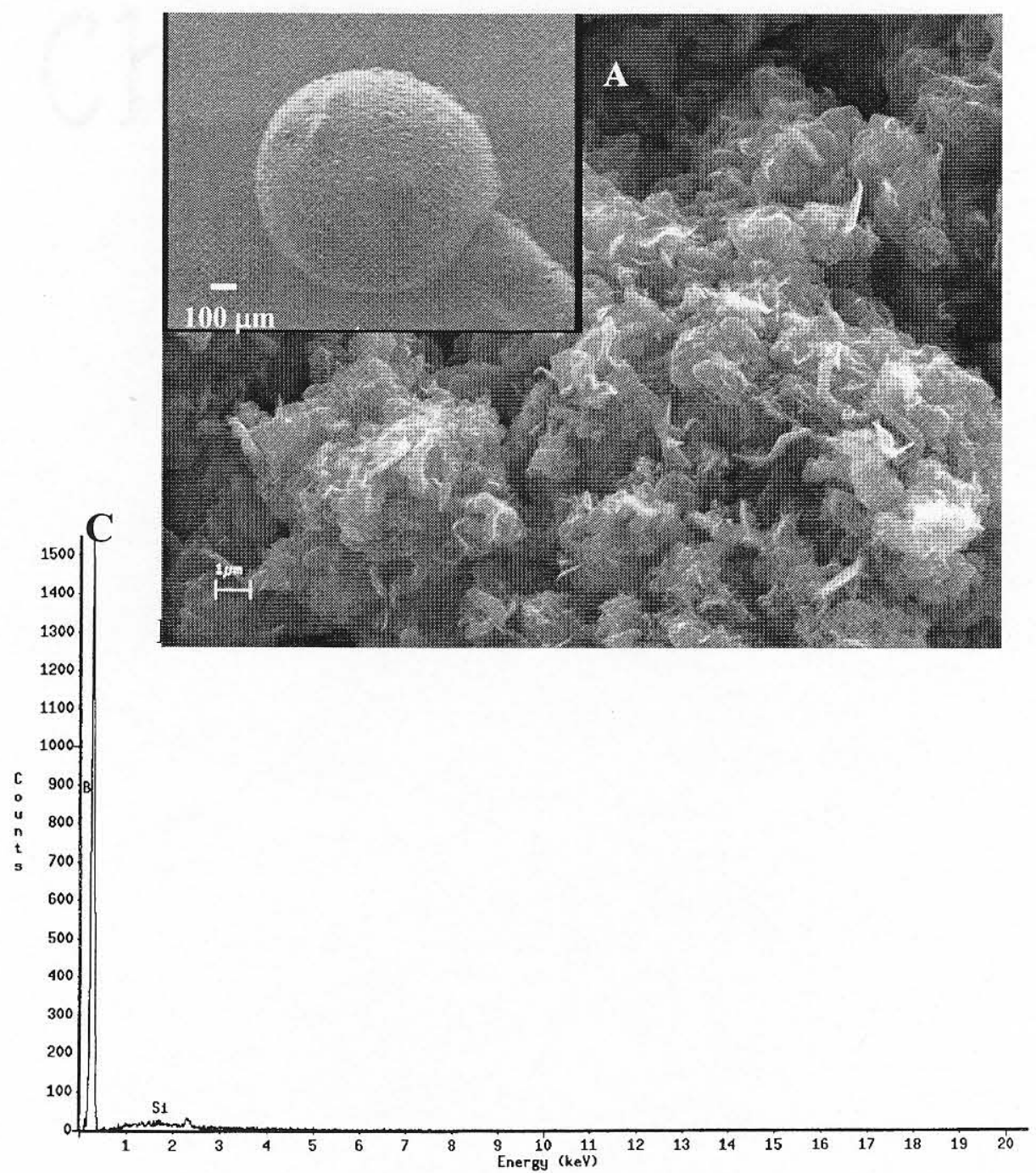

Figure 7.1 (a) SEM image of the ball-shaped deposit on the platinum wire (inset shows the overall tip). (b) EDS of the ball-shaped deposit. 
XRD was performed on the as-synthesized deposit, by carefully scraping the top few layers and placing them on a sample holder. The powder diffraction pattern is shown in Figure 7.2. The main peak $26.25^{\circ}$ corresponds to the (002) plane of graphite.

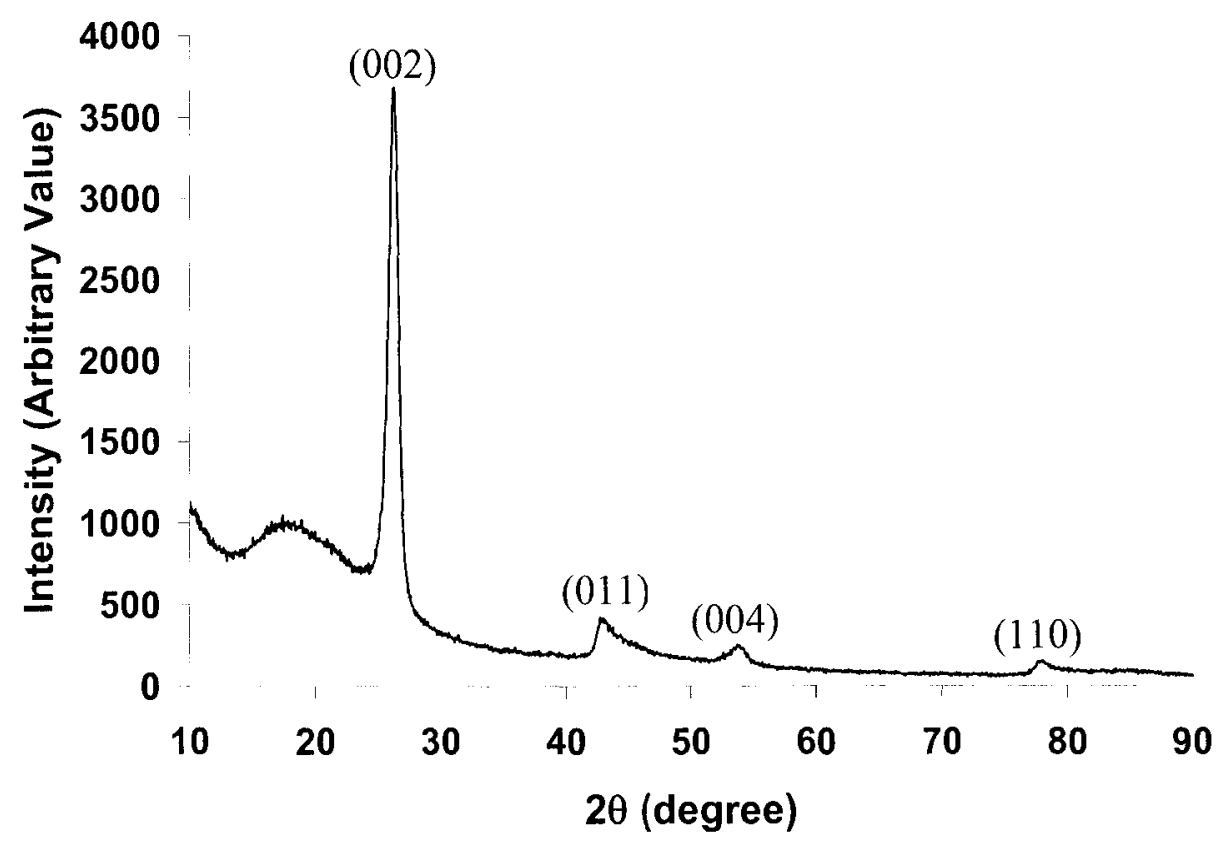

Figure 7.2 XRD spectrum of the scraped ball-shaped deposit (the values in the brackets indicate the crystallographic planes corresponding to the respective peaks).

The full width at half max (FWHM) for this peak is $0.0164 \mathrm{rad}$. The copper wavelength used was 1.54 A. Using Scherrer's formula (Eq. 3.3), the graphite crystal size was estimated to be $9.4 \mathrm{~nm}$. Peaks were observed at $44.7^{\circ}, 54.6^{\circ}$ and $77.5^{\circ}$, which correspond to (011), (004) and (110) of graphite. Clearly, at least on the surface, this material did not contain any diamond phase. However, as mentioned in Chapter 3, the diamond crystals comprised of nearly $1 / 3^{\text {rd }}$ the sample, and were mostly found in the bulk of the deposit, embedded in an amorphous matrix. 
Raman spectra taken at different wavelengths on the nanocomposite material are presented in Figure 7.3. A number of studies have indicated that the presence of the two peaks one around $1350 \mathrm{~cm}^{-1}$ and the other around $1580 \mathrm{~cm}^{-1}$ are clearly indicative of D and $\mathrm{G}$ band of graphite [199]. An additional peak at $1620 \mathrm{~cm}^{-1}$ is indicative of the D'band [200]. The D and D'- band arise due to disorder in the graphite, i.e. perfect graphite sheets would yield only the G band [199]. Both the position and the intensity of the Dband depend on the crystallite size and the laser excitation wavelength. With decreased excitation wavelength D-bands shift to higher wave numbers and decrease in intensity. Raman spectroscopy is very sensitive to the presence of $\mathrm{sp}^{2}$ carbon in comparison to $\mathrm{sp}^{3}$ carbon [201]. Thus, for materials potentially containing both diamond and graphite crystalline phases, short laser wavelengths $(\sim 244 \mathrm{~nm})$ must ideally be used to distinguish between the diamond signature at $1332 \mathrm{~cm}^{-1}$ band and the D-band close to $1350 \mathrm{~cm}^{-1}$ [173]. Decreasing the wavelength from $785 \mathrm{~nm}$ to $488 \mathrm{~nm}$ shifts the D-band from 1313 $\mathrm{cm}^{-1}$ to $1376 \mathrm{~cm}^{-1}$, while the G-band at $1583 \mathrm{~cm}^{-1}$ remains relatively constant. The dependence of the position of D-band on the laser energy (which is obtained from the laser wavelength) is indicated in the inset of Figure 7.3. The D-band changes the wave number position by $50 \mathrm{~cm}^{-1} / \mathrm{eV}$, consistent with previous reports [173-175, 199]. 


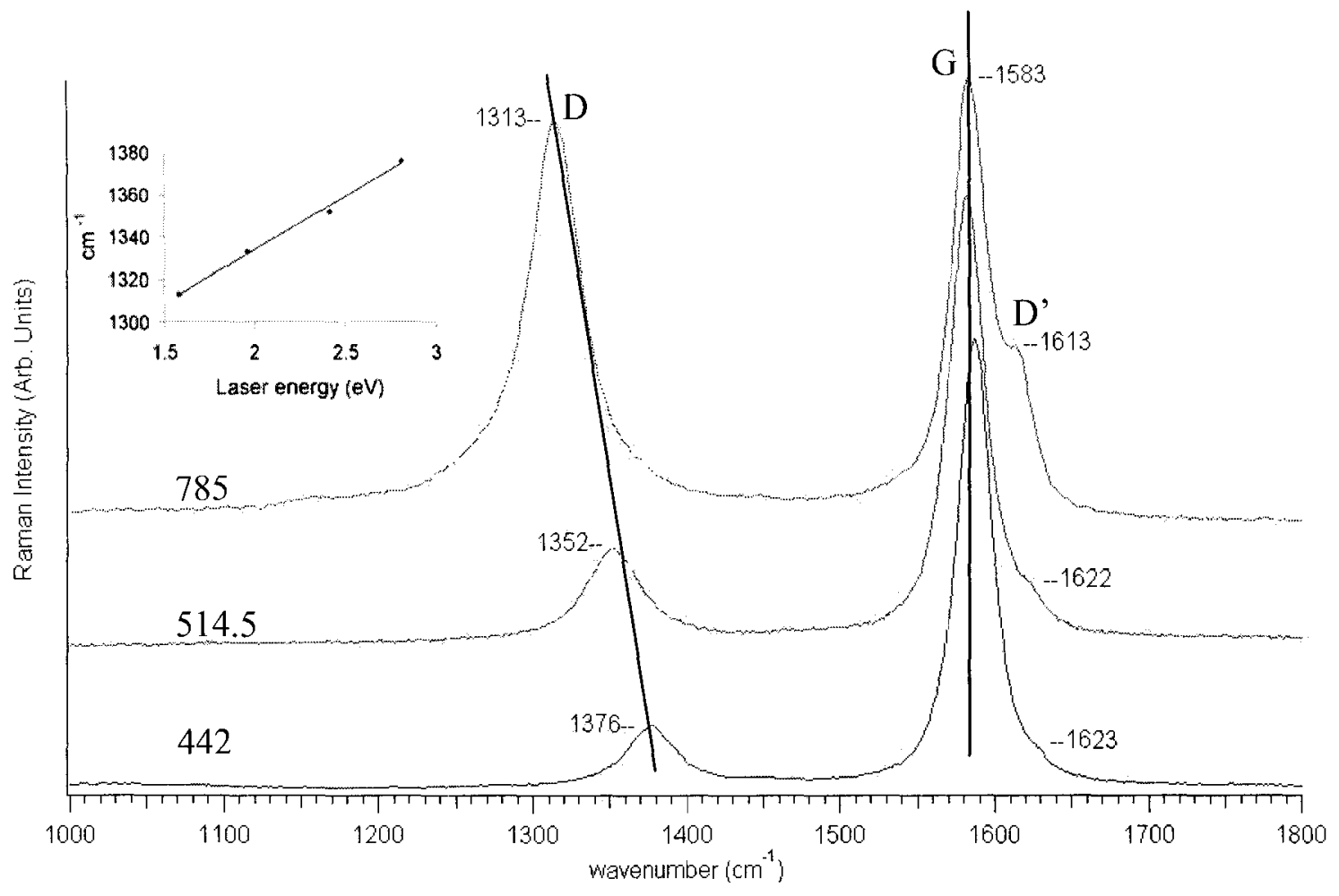

Figure 7.3 Micro-Raman spectra taken from the ball-shaped nanocomposite deposit using different laser excitation wavelengths of $785 \mathrm{~nm}, 514.5 \mathrm{~nm}$, and $442 \mathrm{~nm}$. The inset shows the dependence of D-band wavelength on the laser energy, with a slope of $50 \mathrm{~cm}^{-1}$ / eV.

The relation between the ratio of the $\mathrm{D} / \mathrm{G}$ band intensity and the graphite cluster size $[174,175,199]$ according to Knight's formula is:

$\mathrm{L}_{\mathrm{a}}=\mathrm{C}\left(\mathrm{I}_{\mathrm{G}} / \mathrm{I}_{\mathrm{D}}\right)$

Where $L_{a}$ is the cluster size of graphite, $I_{G}$ and $I_{D}$ are the $G$ - and D-band intensities, and $C$ is a wavelength dependent term. If one were to use a linear relation of $\mathrm{C}$ for the dependence on wavelength as suggested by Matthews et al. [175] in the range 400-785 $\mathrm{nm}$, the value of $\mathrm{C}$ for a wavelength of $633 \mathrm{~nm}$ and $785 \mathrm{~nm}$ would be $\sim 83 \AA$ and $\sim 133 \AA$. The integrated intensity ratio $\left(\mathrm{I}_{\mathrm{G}} / \mathrm{I}_{\mathrm{D}}\right)$ calculated for the nanocrystalline material at laser 
excitation wavelengths $633 \mathrm{~nm}$ was 1.147 and at $785 \mathrm{~nm}$ was 0.68 (See Figure 7.4). Using Knight's formula the crystallite size obtained for the nanocomposite material is lesser than $10 \mathrm{~nm}$. A similar crystal size for $514 \mathrm{~nm}$ and $422 \mathrm{~nm}$ laser wavelengths was obtained. For a Raman laser with wavelength $785 \mathrm{~nm}$, the expected penetration depth is lesser than $1 \mu \mathrm{m}$. This indicates that at least the surface of the nanocomposite material is dominated by nanocrystals of graphite.
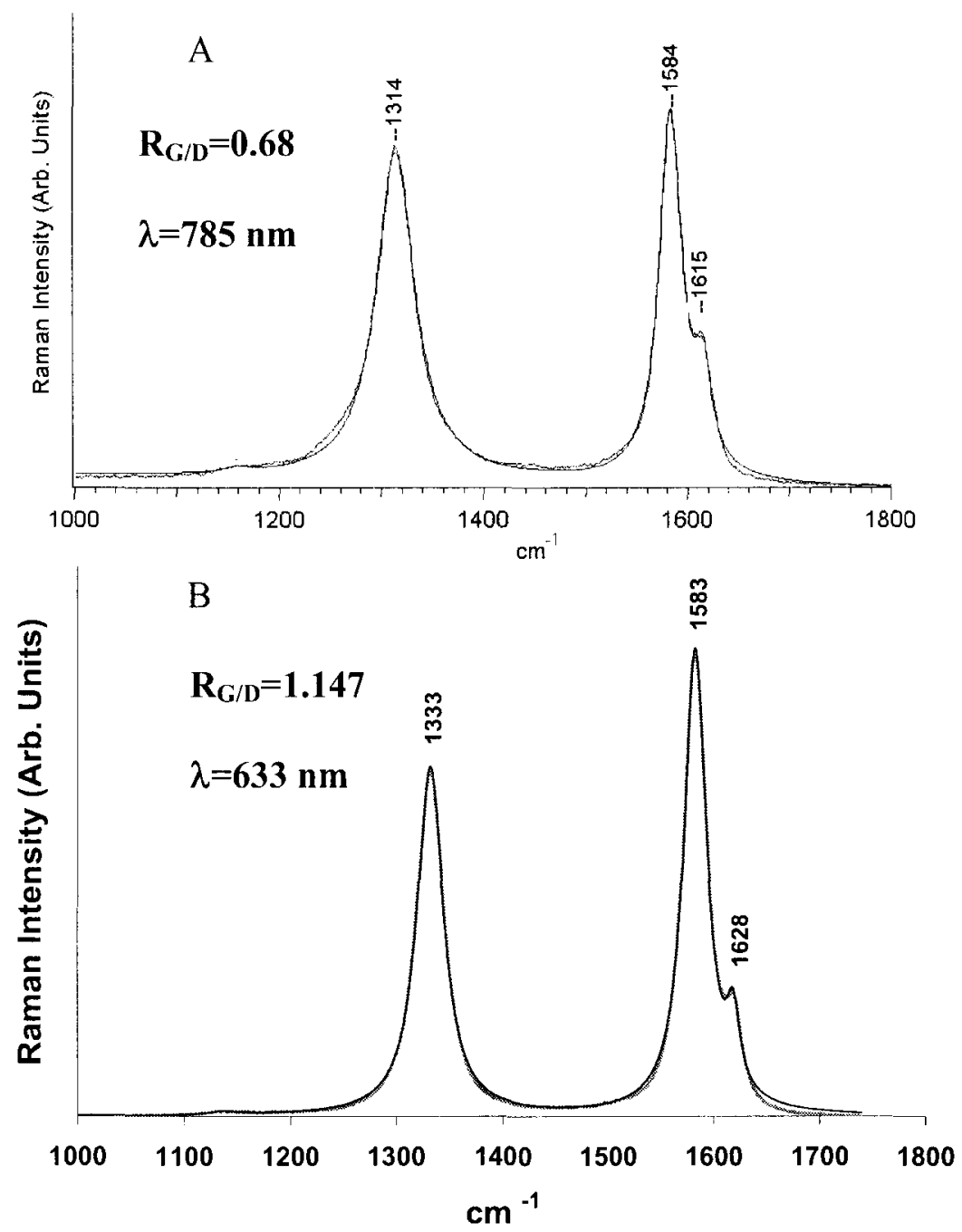

Figure 7.4 Raman spectra with calculated integrated intensity ratio for G/D band (R) for laser wavelengths of (a) $785 \mathrm{~nm}$ (b) $633 \mathrm{~nm}$. 
Nanodiffraction studies were also performed on $\sim 1 \mathrm{~nm}$ regions of the ball-shaped tip. The bulb was taken intact and mounted on a grid. The STEM observations illustrated that the bulb was relatively smooth, and gives graphite diffraction patterns consistent with the basal plane oriented roughly parallel to the surface. The dark field images of the nanocrystals along with their corresponding electron nanodiffraction patterns (taken from $1 \mathrm{~nm}$ regions) are shown in Figure 7.5. The dark-field image shows that the graphite crystals were often only as small as $10 \mathrm{~nm}$ in diameter with sharp edges. The observed graphite crystallite size agreed with the value obtained by Raman analysis. The diffraction patterns showed abrupt changes in lattice orientation between the crystallites.

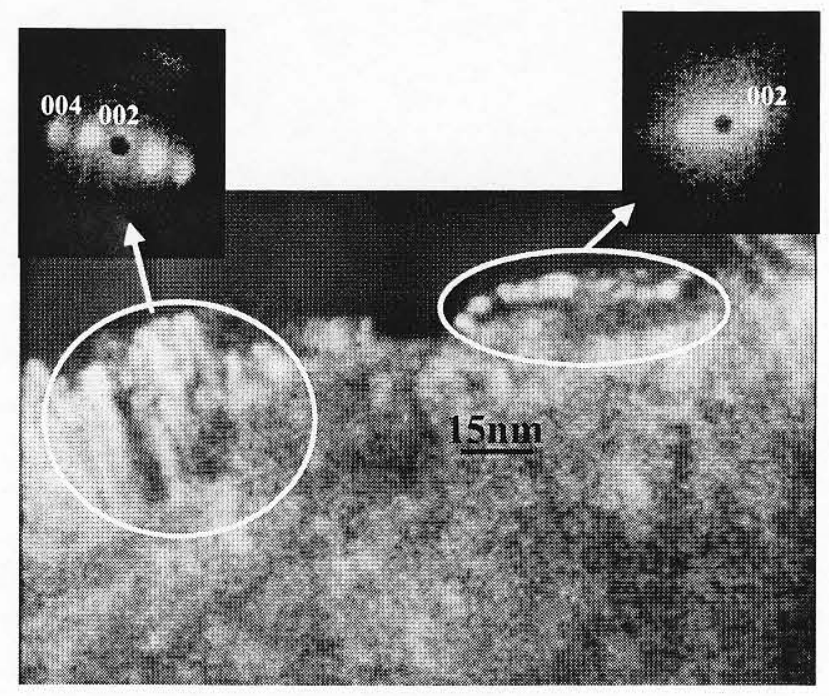

Figure 7.5 Dark field image taken from the intact bulb-part of the electrode. Corresponding electron nanodiffraction patterns indicated in the insets are from $1 \mathrm{~nm}$ region. 
The right hand inset in Figure 7.5 shows only strong first order reflections indicative of very small distorted crystals. This may be due to the presence of many exposed graphite edge planes. When the samples were crushed and the bulk phase of the material was analyzed by STEM, almost a third of the crystallites appeared to be graphitic. In addition, there was a significant amount of amorphous material. However, the proportion of the diamond phase present was quite small $\left(1 / 3^{\text {rd }}\right)$ in comparison to the amount of graphite. The diamond crystals were mostly embedded within the amorphous matrix.

Thus, XRD, Raman spectroscopy and electron nanodiffraction indicated that the deposit surface was covered with graphite crystals whose size was about $10 \mathrm{~nm}$. From now on, this material will be referred to as "nanocrystalline graphite" (NCG). Because this material had nanocrystals of graphite, it was expected that its structure would be dominated by edge planes, and hence would be expected to demonstrate surface electroanalytical properties similar to edge planes of graphite.

\subsection{Electroanalytical chemistry at NCG}

Since the nanocrystalline graphite films and carbon nanopipettes synthesized were already deposited on platinum wires, and hence already had an electrical contact, it was convenient to measure electron transfer reactions on its surface. The following were the series of investigations carried out on NCG and carbon nanopipettes. 


\subsubsection{Working potential window in aqueous solutions}

At sufficiently high over-potentials, gas-evolution (oxygen, hydrogen or chlorine) reactions occur at the electrode-electrolyte interface. The potential range over which no gas-evolution occurs is called the working potential window. A wide potential window allows the detection of molecules oxidizing at high positive potential, which may not be detected with high sensitivity due to the interference of gas evolution. Figure 7.6 shows the Cyclic Voltammetry (CV) behavior in $\mathrm{H}_{2} \mathrm{SO}_{4}$ obtained for $\mathrm{NCG}$ electrode in comparison with that for $\mathrm{MCD}$ and $\mathrm{GC}$. The potential window for nanocrystalline diamond is relatively broad, with the potential range extending from approximately +1.8 $\mathrm{V}$ to $-1.1 \mathrm{~V}$ (vs. $\mathrm{Ag} / \mathrm{AgCl}$ ). This was a slightly narrower range by $200-300 \mathrm{mV}$ than obtained for MCD or GC.

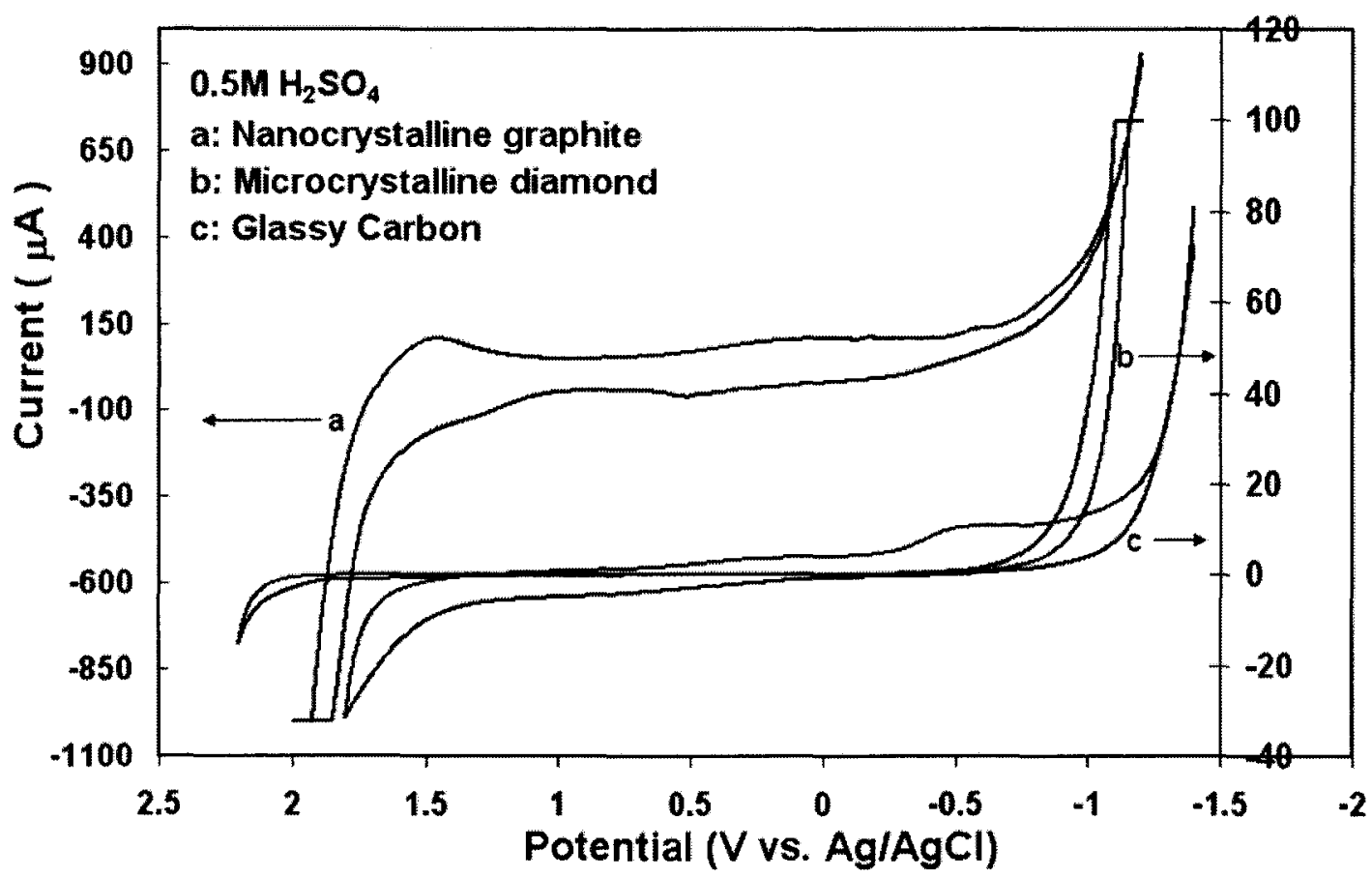

Figure $7.6 \mathrm{CV}$ in $0.5 \mathrm{M}$ sulfuric acid solution at $100 \mathrm{mV} / \mathrm{s}$ scan rate at the (a) $\mathrm{NCG}$, (b) MCD, and (c) GC electrode. 
The reason for high overpotentials for hydrogen and oxygen evolution at diamond was due to the low interaction of diamond surface with the dissolved species, consistent with hydrogen termination of the surface of CVD-diamond. With the increase of $\mathrm{sp}^{2}$ carbon at the grain boundaries, the potential window reduces [182]. Since nanocrystalline graphite is dominated by $\mathrm{sp}^{2}$ sites, the lower potential window could be attributed to these sites.

\subsubsection{Background current and double layer capacitance}

Graphite is a semimetal, with properties similar to that of a semiconductor. When a semi-conductor comes in contact with an electrolyte, electric current initially flows across the junction until electronic equilibrium is reached, where the Fermi level of the solid electrode $\left(E_{\mathrm{f}}\right)$ equals the redox potential of the electrolyte $\left(\mathrm{E}_{\mathrm{rcdox}}\right)$. The transfer of electric charge from electrolyte to the solid electrode produces a region on either side of the interface, where the charge distribution differs from the bulk material [202]. This region is called the space-charge layer on the electrode side and double-layer (Helmholtz) on the electrolyte side, which is followed by the diffuse layer (see Section 3.5.2).

In electroanalysis, the current observed in blank electrolyte solutions (in absence of any analyte) has several components, including double-layer charging, redox reactions of the electrode surface (like surface oxide formation), and redox reactions of the impurities in the electrolyte solution, particularly oxygen [177]. The most important contribution comes from the double-layer charging current. This charging current generally increases linearly with scan-rate for a planar or macroscopic electrode, depending on the type of surface. Low background current is desirable for detection of 
analytes at low concentration, with the consequence of improving signal to background $(\mathrm{S} / \mathrm{B})$ ratio. As seen in Figure 7.6, MCD has exceptionally low (an order of magnitude) background current due to its lower double layer capacitance, in comparison to NCG and GC, which have a higher value of double-layer capacitance. Forms of carbon other than diamond may exhibit a non-classical form of charging current that is greater than the double-layer effect alone. This is attributed to surface components such as ion adsorption, porosity, or oxidation of the surface. However, all these components are clubbed into the general "capacitance" reported for these materials. Capacitance values will be discussed in the EIS measurements in Section 7.3.4.

In electroanalytical sensing, signal vs background is important, and it will be evident from the following discussions that the signal obtained at NCG is significant even though it has a high background current, thus making the ratio of S/B sufficiently high.

\subsubsection{Ferricyanide redox reactions}

In general, electron exchange reactions are classified as outer sphere and inner sphere reactions. The former involve outer coordination sphere of reacting ions, while the inner co-ordination sphere remains untouched by the electron transfer, and also there are no breaking or forming of bonds. The inner-sphere electron transfer is accompanied by breaking or forming of interatomic bonds. Thus, outer-sphere reactions are less sensitive to the type of surface. Potassium Ferricyanide $\mathrm{K}_{3} \mathrm{Fe}(\mathrm{CN})_{6}$ is one of the wellstudied outer-sphere electrochemical reactions on carbon surface. However, this analyte is sensitive to the type of carbon surface. 
Figure 7.7 shows the response of $\mathrm{NCG}$ to $1 \mathrm{mM} \mathrm{Fe}(\mathrm{CN})_{6}^{3-14-}$ in $0.1 \mathrm{M} \mathrm{KCl}$ at different scan rates from $20 \mathrm{mV} / \mathrm{s}$ to $200 \mathrm{mV} / \mathrm{s}$. The $\Delta \mathrm{Ep}$ is remains constant at $82 \mathrm{mV}$ throughout the $\mathrm{CV}$ runs at different scan rates. Since this reaction is a single-electron transfer reaction, according to Nernst equation, the peak separation should be $\Delta \mathrm{Ep}=59 / \mathrm{n}$ $=59 \mathrm{mV}$. However, the response to $\mathrm{Fe}(\mathrm{CN})_{6}{ }^{3-14}$ is very dependent on the type of carbon surface. Hence NCG shows a quasi reversible response. In comparison, diamond shows a peak separation of $88 \mathrm{mV}$ [181], GC shows a wide range from $64 \mathrm{mV}$ for a fractured surface [177] to $120 \mathrm{mV}$ for surface pre-treated with organic solvents [203]. However, for glassy carbon, the $\Delta \mathrm{Ep}$ changes with exposure to air for a freshly cleaved surface. NCG and MCD on the other hand have a stable response. Basal plane of graphite shows $>700 \mathrm{mV}$ peak separation [204]. 


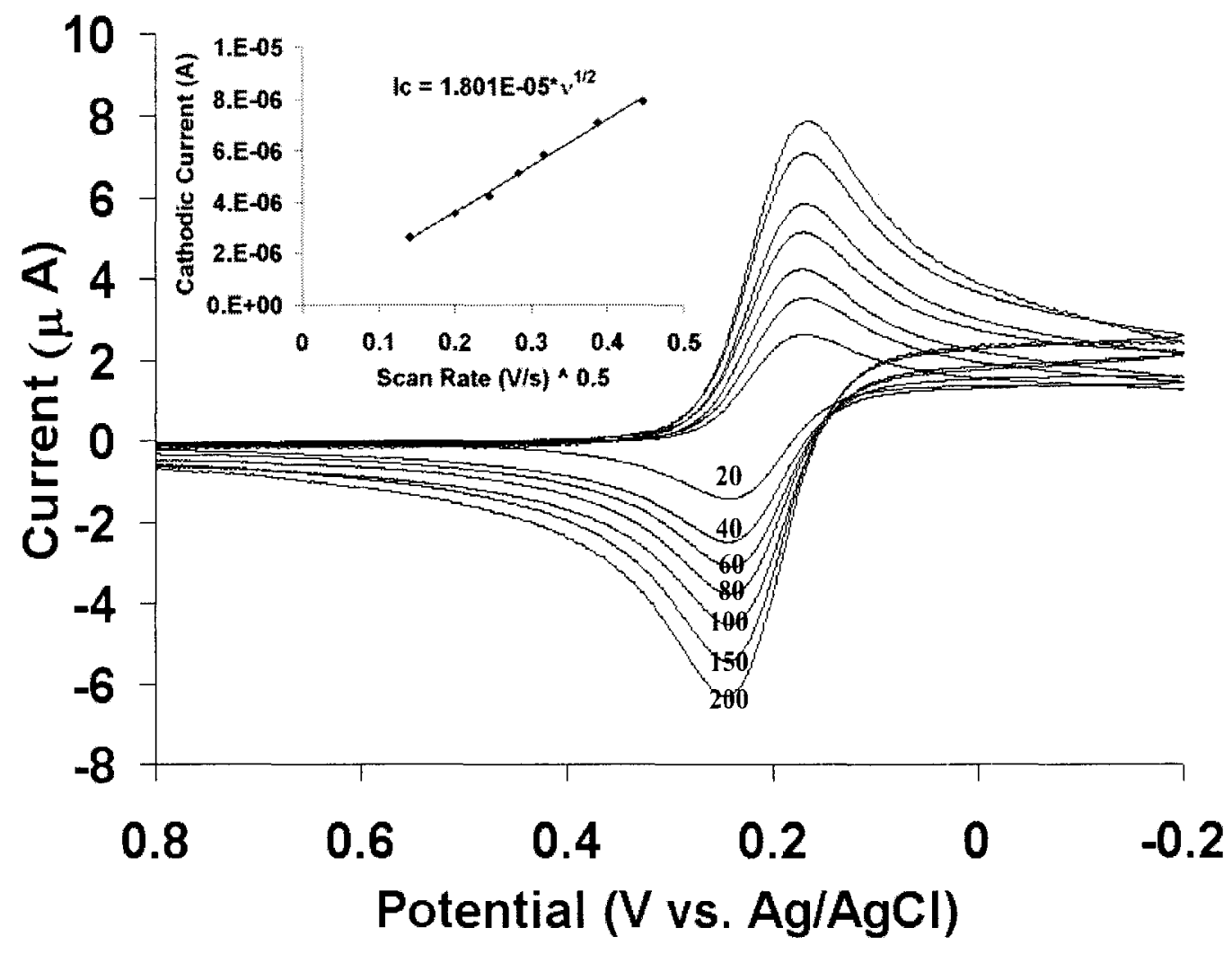

Figure $7.7 \mathrm{CV}$ of $1 \mathrm{mM} \mathrm{K}{ }_{3} \mathrm{Fe}(\mathrm{CN})_{6}$ in $0.1 \mathrm{M} \mathrm{KCl}$ solution at scan rates from $20 \mathrm{mV} / \mathrm{s}$ to $200 \mathrm{mV} / \mathrm{s}$ at NCG. Inset shows that the variation of cathodic current with scan rate ${ }^{\wedge} 0.5$ is linear.

In a CV, initially, both the surface of the electrode and the solution are at the same bulk concentration of the analyte. As the potential is scanned across the electrode, the analyte begins to get consumed due to electron transfer reaction only when the $E_{0}$ is reached. The current is proportional to the mass flux of the analyte at the surface. Due to the consumption by reaction, more of the analyte is transported to the electrode surface from the bulk solution. Hence, a diffusion boundary layer is set-up in the vicinity of the electrode surface due to the concentration gradient. The thickness of this boundary layer 
depends on the geometry of the electrode. For a planar electrode, the thickness of this boundary layer increases with time, and the concentration of the analyte at the surface decreases with time. Eventually, when E $>$ Eo (high over potentials), the electron transfer is governed by the mass transport of the analyte to the surface. This leads to a decrease in current, and hence a peak shaped behavior of the current Vs E. When the mass transport to the surface of the electrode is solved using Fick's law, the current (i) dependence with time for planar diffusion is given by the famous Cottrell equation:

$i(t)=\frac{n F A D C}{(\pi D t)^{0.5}}$

Where, $\mathrm{n}$ is the number of electrons transferred, $\mathrm{F}$ is Faraday's constant (96487 C), A is the area of the electrode, D is the diffusion coefficient of the analyte in the solution, and $\mathrm{C}$ is the concentration of the analyte in the bulk. Thus, current is inversely proportional to the square root of time $(\mathrm{t})$. Time for a cyclic voltammogram is given by $(\mathrm{RT} / \mathrm{nFv})$, where $\mathrm{R}$ is the gas constant, $\mathrm{T}$ is the temperature, and $v$ is the scan rate. This leads to the Randles-Sevick equation:

$i_{p}=\left(2.69 \times 10^{5}\right) n^{3 / 2} A C D^{1 / 2} v^{1 / 2}$

Where, $\mathrm{i}_{\mathrm{p}}$ is the peak current (anodic or cathodic) in $\mathrm{A}$, and the units of $\mathrm{A}$ is $\mathrm{cm}^{2}, \mathrm{C}$ is in $\mathrm{mol} / \mathrm{cm}^{3}, \mathrm{D}$ is in $\mathrm{cm}^{2} / \mathrm{s}$ and $v$ is in $\mathrm{V} / \mathrm{s}$. The plot of $\mathrm{i}_{\mathrm{p}} \mathrm{vs} v^{1 / 2}$ should be a straight line if the current is just limited by the diffusion of species to the electrode surface (i.e. not limited by kinetics of the reaction or any competing process). The inset in Figure 7.7 shows that the cathodic current (since $\mathrm{Fe}(\mathrm{CN})_{6}{ }^{3-14-}$ first gets reduced) depends linearly with the square-root of the scan rate, indicating that the reaction is diffusion controlled. 
The slope of the line in the inset of the Figure 7.7 gives the geometrical area, A, by taking the value of $\mathrm{D}$ reported in literature. This experiment was used to determine the geometrical area of all electrodes.

\subsubsection{Detection of catechols and quinones}

The as-synthesized electrode material contains nanocrystalline graphite, which may possibly have high fraction of edge planes. Hence, it is expected to have good electron transfer rates for catechols and quinones, whose electrochemistry is catalyzed by their surface adsorption. Figure 7.8(a-f) shows the CVs obtained using the electrode material for a series of model electroactive analytes in $0.1 \mathrm{M} \mathrm{KCl}$ solution at $100 \mathrm{mV} / \mathrm{s}$ scan rate. $0.1 \mathrm{M} \mathrm{KCl}$ was used as the electrolyte in order to compare the $\Delta$ Ep obtained with those reported in the literature. 

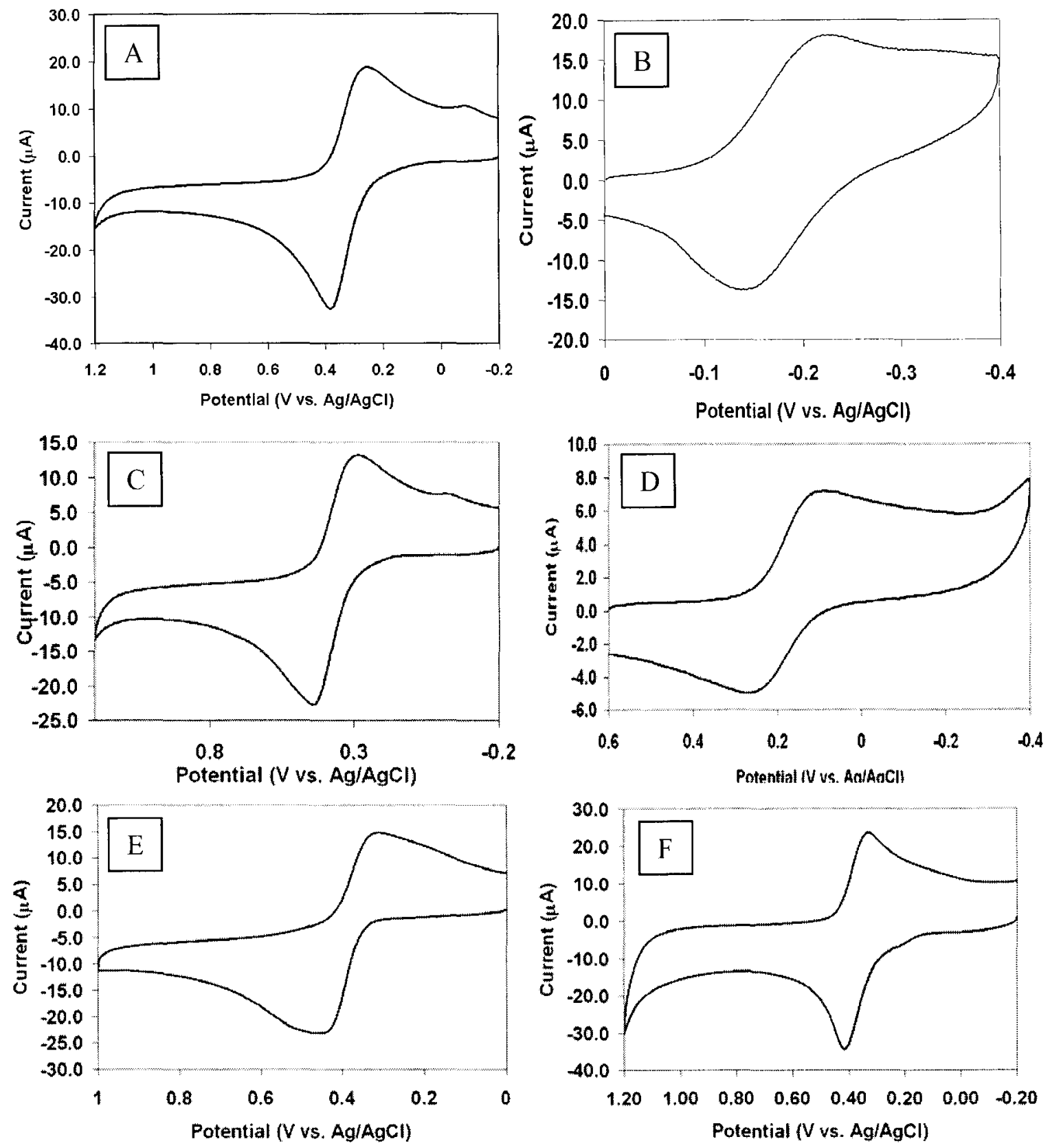

Figure 7.8 CV of $1 \mathrm{mM}$ (a) 4-Methyl catechol, (b) ruthenium hexamine hydrochloride, (c) catechol, (d) ferricyanide, (e) hydroquinone, and (f) dopamine in $0.1 \mathrm{M} \mathrm{KCl}$ solution at $100 \mathrm{mV} / \mathrm{s}$ scan rate at $\mathrm{NCG}$ electrode. 
The specific values of peak separations $(\Delta \mathrm{Ep})$ are listed in Table 7.1 and compared with those obtained using untreated GC and MCD electrodes. In comparison to the near-reversible response seen at the NCG electrode, both untreated GC and MCD gave much less reversible behavior for most of the analytes. These results illustrate that NCG clearly presents as an interesting electrode material for electroanalytical applications.

Table 7.1 Comparison of the $\Delta$ Ep values obtained experimentally for different analytes at untreated GC, MCD and NCG electrode materials.

\begin{tabular}{|l|l|l|l|}
\hline Compounds & $\mathrm{GC}$ & $\mathrm{MCD}$ & $\mathrm{NCG}$ \\
\hline 4-Methyl catechol & $0.49 \mathrm{~V}$ & $1.06 \mathrm{~V}$ & $0.13 \mathrm{~V}$ \\
\hline $\mathrm{Ru}\left(\mathrm{NH}_{3}\right)_{6}{ }^{2+}$ & $0.07 \mathrm{~V}$ & $0.09 \mathrm{~V}$ & $0.08 \mathrm{~V}$ \\
\hline Catechol & $0.55 \mathrm{~V}$ & $1.02 \mathrm{~V}$ & $0.14 \mathrm{~V}$ \\
\hline $\mathrm{Fe}(\mathrm{CN})_{6}{ }^{3+}$ & $0.5 \mathrm{~V}$ & $0.65 \mathrm{~V}$ & $0.08 \mathrm{~V}$ \\
\hline Hydroquinone & $0.55 \mathrm{~V}$ & $1.2 \mathrm{~V}$ & $0.21 \mathrm{~V}$ \\
\hline Dopamine & $0.65 \mathrm{~V}$ & $1.0 \mathrm{~V}$ & $0.12 \pm 0.02 \mathrm{~V}$ \\
\hline Ascorbic Acid & $1.2 \mathrm{~V}^{*}$ & $1.6 \mathrm{~V}$ & $0.21 \mathrm{~V}^{*}$ \\
\hline
\end{tabular}

* Oxidation irreversible at all electrode materials

A series of tests were employed on several electrodes to gain more insight into the electrochemical activity of the NCG. The electrodes were tested with regard to stability, nature of surface sites, and selectivity in mixtures of analytes. In all of these tests, we used dopamine as the model analyte due to its importance as a neurological fluid. 


\subsubsection{Dopamine redox reactions}

Dopamine is a very important neurological compound found in neural cells. Deficiency of dopamine leads to Parkinson's disease [205]. As a treatment, patients of Parkinson's disease are given doses of dopamine. The effect of the dopamine dosage eventually falls after a few hours and thus detecting dopamine levels in vivo is essential to know when the next dosage becomes necessary. The brain is a challenging environment for chemical sensing due to the low concentrations of analytes that need to be detected in presence of a number of interfering compounds.

As shown in Figure 7.9, dopamine gave a nearly reversible $\mathrm{CV}$ with the nanocomposite diamond electrode with a peak separation $\Delta \mathrm{E}_{\mathrm{p}}$ of only $100 \mathrm{mV}$ between the anodic and cathodic waves. The reversible peaks were generally observed without any pre-treatment. This behavior was much more reversible than that for the other electrodes examined as $\Delta \mathrm{E}_{\mathrm{p}}$ values for $\mathrm{GC}$, platinum, and $\mathrm{MCD}$ were $700 \mathrm{mV}, 500 \mathrm{mV}$, and $900 \mathrm{mV}$ respectively ( $\mathrm{GC}$ or $\mathrm{MCD}$ were not pre-treated in any way). 


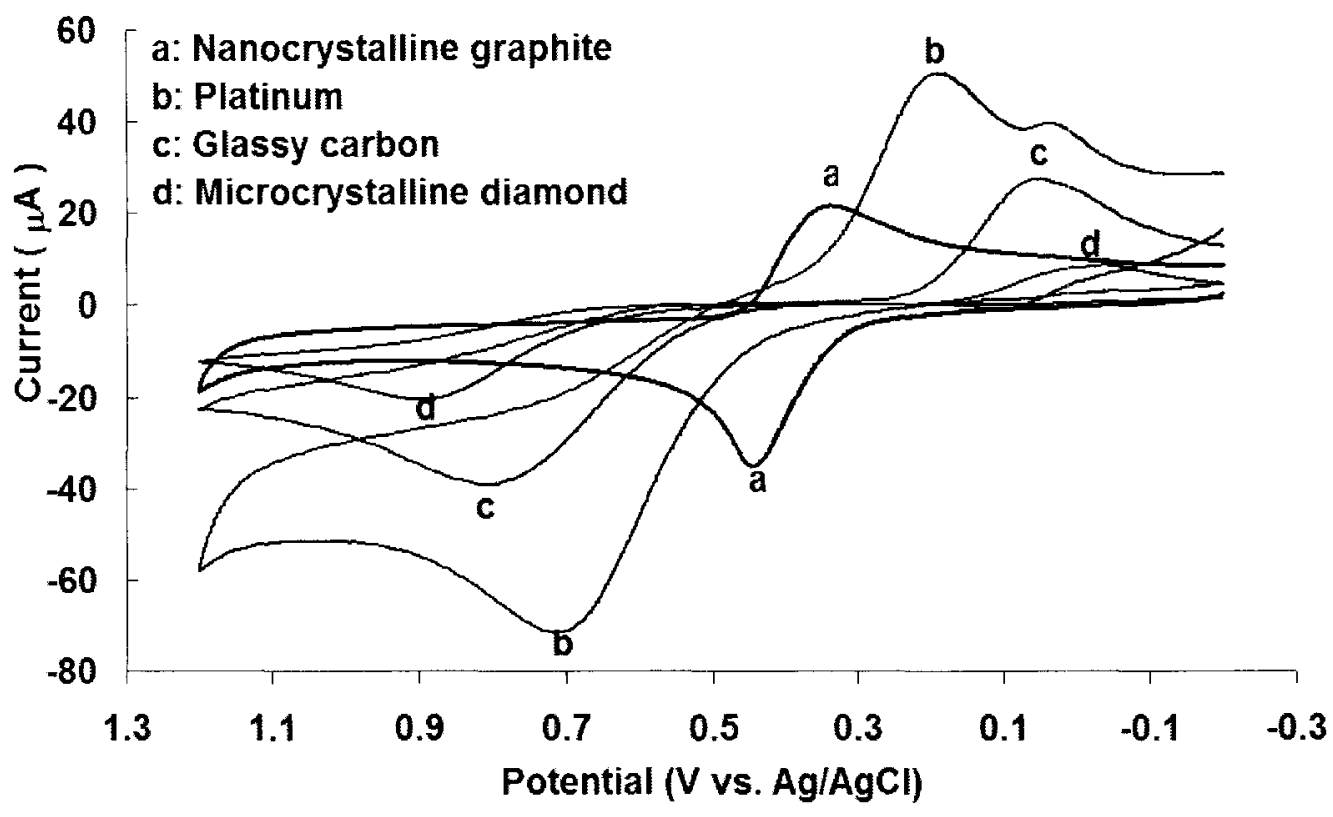

Figure $7.9 \mathrm{CV}$ of $1 \mathrm{mM}$ dopamine in $0.1 \mathrm{M} \mathrm{KCl}$ solution at (a) NCG, (b) Pt, (c) GC, and (d) MCD electrodes at $100 \mathrm{mV} / \mathrm{s}$ scan rate.

Dopamine/Dopamine orthoquinone redox systems follows the "scheme of squares", with two electron transfer steps combined with two fast proton transfer steps [206]: DA $\rightarrow$ DOQ $+2 \mathrm{H}^{+}+2 \mathrm{e}^{-}$. The theoretical peak separation according to Nernst equation should be $59 \mathrm{mV}$. Hence, clearly the response seen for dopamine at NCG was quasi-reversible. The peak currents seen for both anodic and cathodic dopamine waves were found to increase linearly with potential scan rate from $10 \mathrm{mV} / \mathrm{s}$ up to at least 200 $\mathrm{mV} / \mathrm{s}$ with the nanocrystalline graphite film, and the cycling could be continued indefinitely with no change or deterioration observed in the response (Figure 7.10). This indicates that the overall electrochemical process for dopamine at the NCG electrodes is diffusion-controlled. 


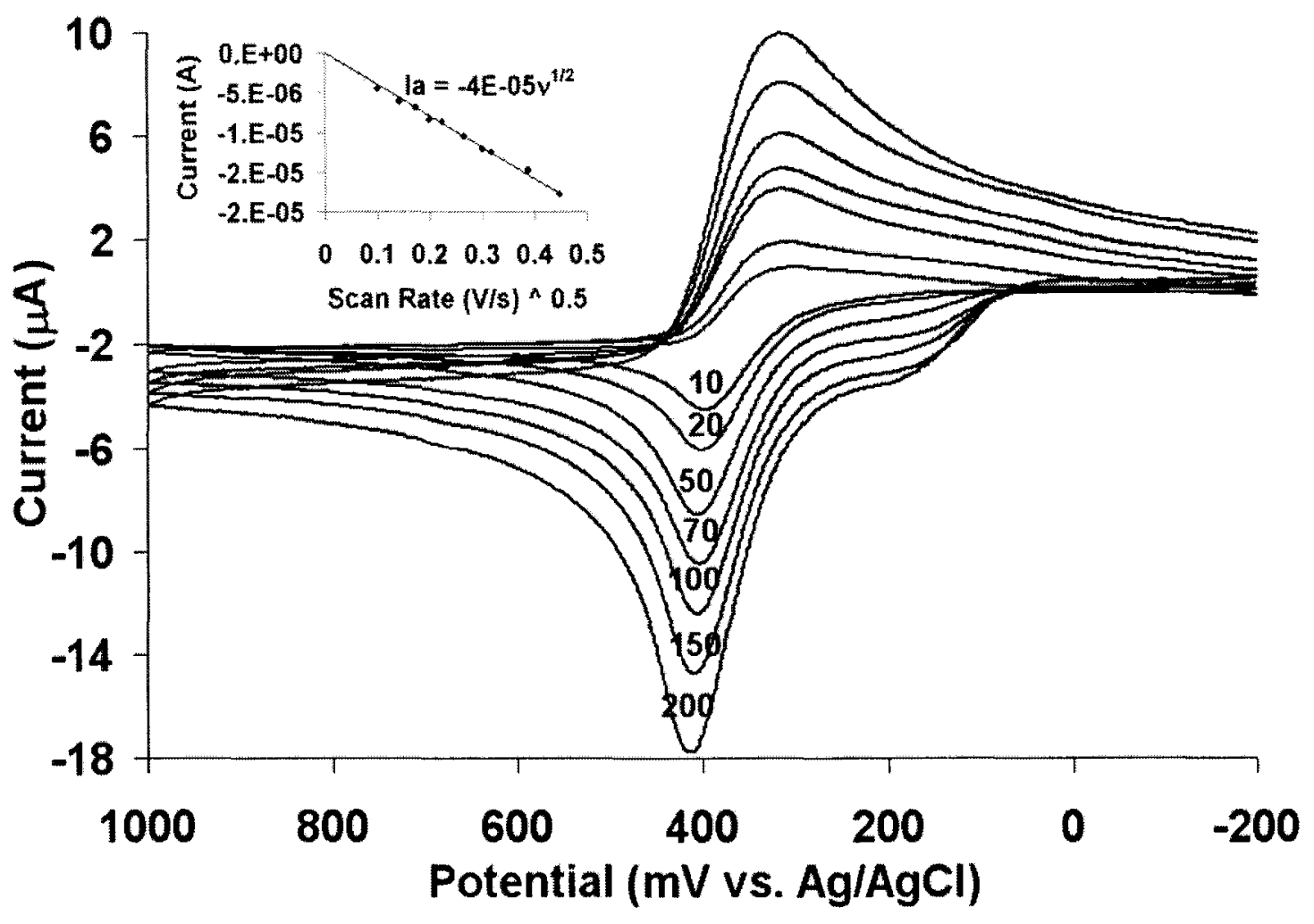

Figure $7.10 \mathrm{CV}$ of $1 \mathrm{mM}$ dopamine in $0.1 \mathrm{M} \mathrm{KCl}$ at different scan rates at $\mathrm{NCG}$ electrode. Inset shows that the variation of anodic current with scan rate ${ }^{\wedge} 0.5$ is linear.

The minimum concentration of dopamine in $0.1 \mathrm{M} \mathrm{KCl}$ solution that could be detected by cyclic voltammetry was in the order of $10 \mu \mathrm{M}$.

\subsubsection{Effect of anodic pretreatment for some electrodes}

The as-synthesized, NCG electrodes showed a quasi-reversible peak separation for dopamine without any pre-treatment $(\sim 100 \mathrm{mV}$ in $0.1 \mathrm{M} \mathrm{KCl}$ solution, and $\sim 60 \mathrm{mV}$ in pH 7 phosphate buffer solution). However, a few of the electrodes (approx. 1 in 5) required anodic oxidation at $+2.0 \mathrm{~V}$ in $0.5 \mathrm{M} \mathrm{H}_{2} \mathrm{SO}_{4}$ in order to reduce the peak separation for dopamine in $0.1 \mathrm{M} \mathrm{KCl}$ from $350 \mathrm{mV}$ to a nearly reversible $100 \mathrm{mV}$ (see 
Figure 7.11). The change in the peak separation indicates the change in the rate controlling electron transfer step, as suggested for the case of catechols [207].

The decrease in peak separation was also accompanied by a general increase in the background current, which was mainly due to an increase in the active surface sites as explained later. However, the signal to background ratio is still comparable to that obtained before anodic oxidation. These electrodes, after the one-time anodic oxidation treatment and the remaining electrodes without pre-treatment, exhibited stable and reversible response with usage over the entire period under consideration, i.e., over a year. $\Delta \mathrm{Ep}$ of about 10 electrodes for $1 \mathrm{mM}$ dopamine in $0.1 \mathrm{M} \mathrm{KCl}$ solutions was $0.12 \mathrm{~V}$ $\pm 0.02 \mathrm{~V}$.

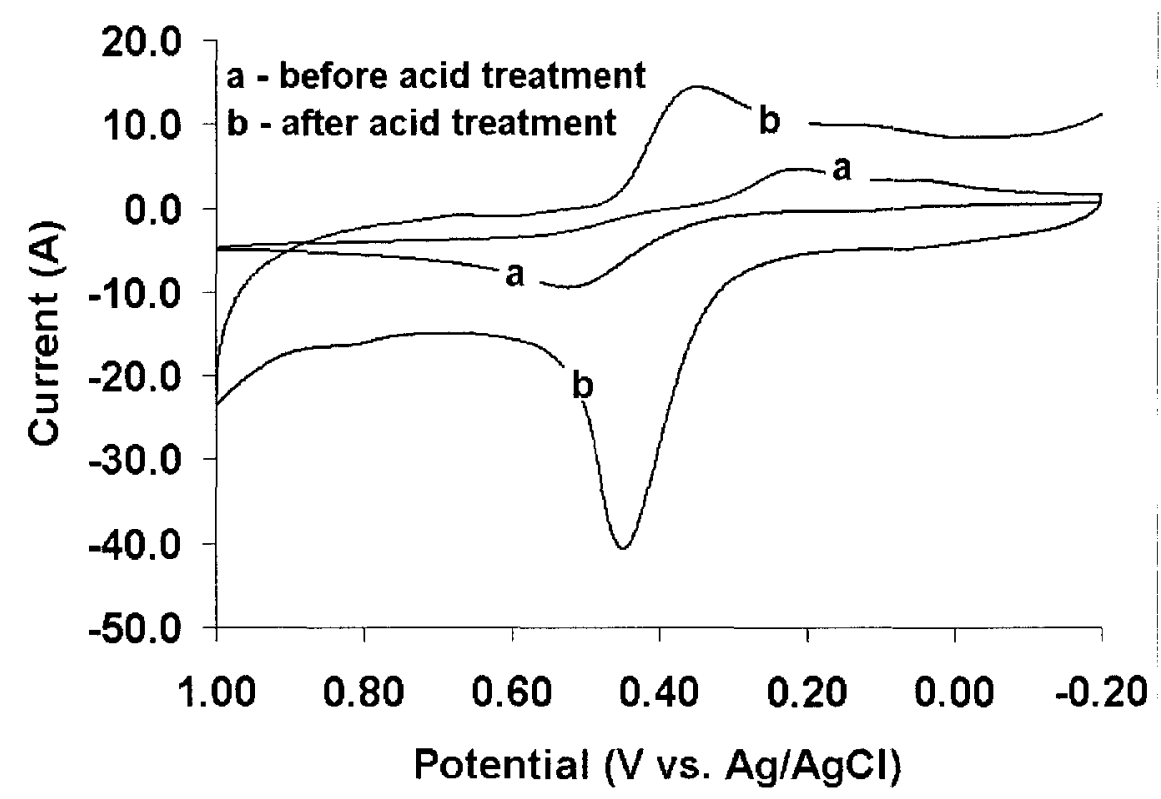

Figure $7.11 \mathrm{CV}$ of $1 \mathrm{mM}$ dopamine in $0.1 \mathrm{M} \mathrm{KCl}$ at $100 \mathrm{mV} / \mathrm{s}$ scan rate at NCG electrode before and after acid treatment. 


\subsubsection{X-ray photoelectron spectroscopy (XPS) investigations}

In order to understand the effect of surface pre-treatment by anodic oxidation on NCG, XPS measurements were performed. McCreery's pioneering studies demonstrated that the electron transfer kinetics increases with increasing adsorption of catechols on $\mathrm{GC}$ surfaces [208]. There are several ways by which catechols can adsorb on the surface of carbon electrodes. One adsorption pathway is through oxygen functionalization of the surface. An example is the formation of $-\mathrm{COOH}, \mathrm{C}=\mathrm{O}$ on the surface of diamond [182], or GC [207], through anodic oxidation. The electrostatic interactions between the oxygen-terminated surface and the catechols lead to increased adsorption of catechols. Electron transfer then occurs from the catechol species in the solution to the surface already saturated with adsorbed oxidized and reduced species [208]. The second pathway to increased adsorption is by means of surface defect sites on graphite i.e. edgeplanes [209]. Surface spectroscopy was used to identify surface functionalities in order to determine which of the above pathways was primarily responsible for the quasireversible behavior seen for dopamine at NCG.

The XPS analysis was performed both on as-synthesized electrodes that provided an immediate reversible response to dopamine and on non-ideal electrodes (before and after electrochemical anodization) that showed slight irreversibility when used assynthesized. The true quantification of oxygen or oxide functionalities at the surface was difficult to determine due to the extremely small area of the electrode in comparison to the region over which the spectrum was taken. The $\mathrm{C}(1 \mathrm{~s})$ from chemical treatment of identical electrodes is presented in Figure 7.12. The $\mathrm{C}(1 \mathrm{~s})$ window can provide additional useful information on chemical state after appropriate deconvolution of the 
data. A simple Gaussian model was used to analytically extract spectral features. As shown in Figure 7.12, three main features were seen, $284.9 \mathrm{eV}, 286.36 \mathrm{eV}, 288.28 \mathrm{eV}$, which have been interpreted elsewhere as $\mathrm{C}-\mathrm{C}$ graphite carbon ring, $\mathrm{C}-\mathrm{OH}$ and $\mathrm{C}=\mathrm{O}$ respectively $[210,211]$.

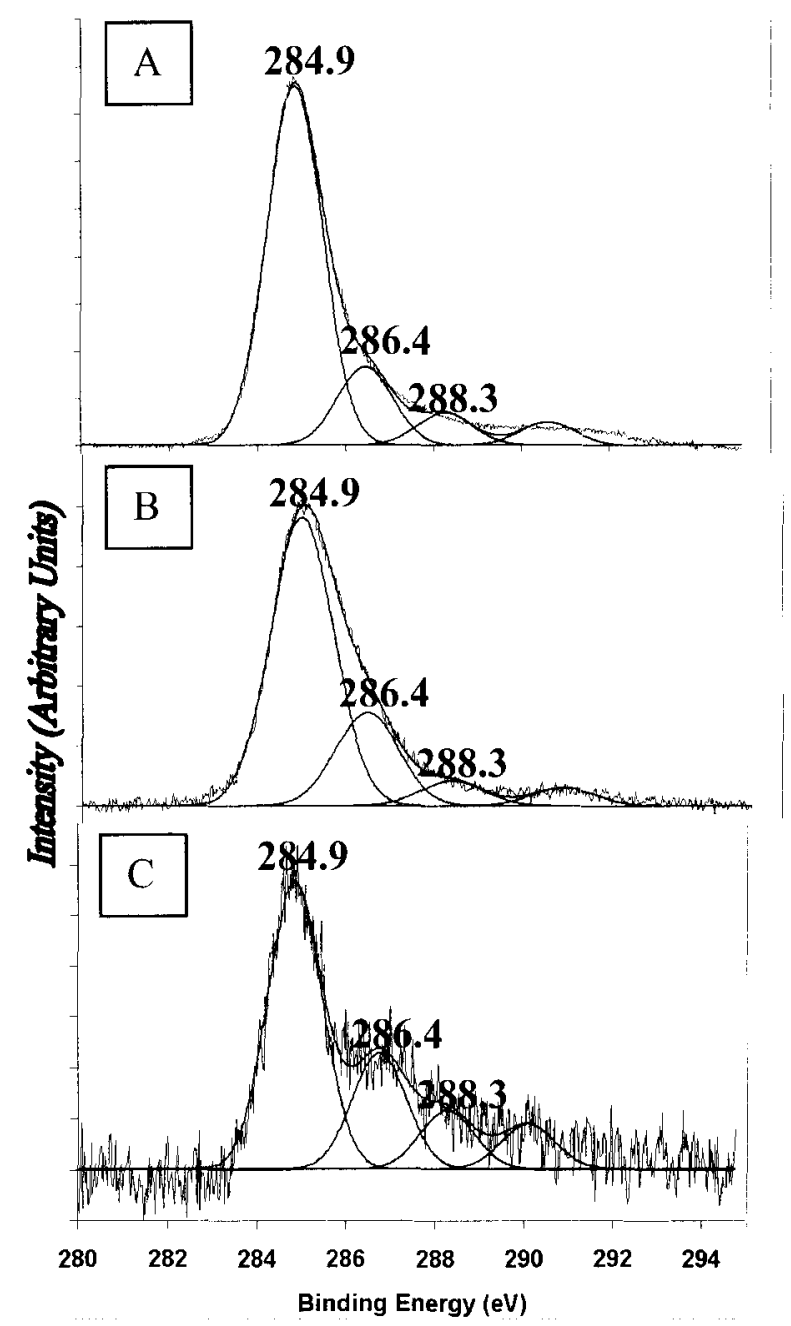

Figure 7.12 XPS spectra for C(1s) taken on the (a) as-synthesized well-working NCG electrode, (b) as-synthesized NCG electrode with irreversible behavior, and (c) after anodic oxidation of (b) in $0.5 \mathrm{M} \mathrm{H}_{2} \mathrm{SO}_{4}$ at $2.0 \mathrm{~V}$ 
Table 7.2 presents the relative composition of the deconvoluted $\mathrm{C}(1 \mathrm{~s})$ spectra in Figure 7.12. It can be seen that with oxidation, the amount of carbonyl functional group increases significantly (column (b) to (c) of Table 7.2). This is consistent with that previously reported for GC [212], graphite [213, 214] and diamond [182,215]. The electrodes with poor and good performance before any electrochemical treatment show a similarity in terms of fractional surface functional groups. Hence, the oxygen-containing surface species coverage was clearly not responsible for the reversible electrochemical behavior.

Table 7.2 Deconvoluted $\mathrm{C}(1 \mathrm{~s})$ peak of the XPS spectrum obtained from the NCG electrode material and percentage composition of each functional group

\begin{tabular}{|l|l|l|l|}
\hline $\begin{array}{l}\text { Peak position/ } \\
\text { assignment }\end{array}$ & $\begin{array}{l}\text { (a) Electrode with } \\
\text { reversible response }\end{array}$ & $\begin{array}{l}\text { (b) Electrode with } \\
\text { irreversible response }\end{array}$ & $\begin{array}{l}\text { (c) Electrode (b) } \\
\text { anodically oxidized }\end{array}$ \\
\hline $\begin{array}{l}284.9 \mathrm{eV}-\text { graphitic } \\
(\mathrm{C}=\mathrm{C})\end{array}$ & 76.4 & 71 & 62 \\
\hline $\begin{array}{l}286.4 \mathrm{eV}-\text { alcoholic } \\
(-\mathrm{OH})\end{array}$ & 16.7 & 22.8 & 25.3 \\
\hline $\begin{array}{l}288.3 \mathrm{eV}-\text { carbonyl } \\
(-\mathrm{COO})\end{array}$ & 6.9 & 6.2 & 12.7 \\
\hline
\end{tabular}

\subsubsection{Electrode capacitance measurements}

Electro impedance spectroscopy (EIS) measurements were performed to estimate the capacitance of nanocrystalline graphite. These measurements indicated that the capacitance of the as-synthesized NCG material was about $5.5 \mu \mathrm{F}$ for $0.03 \mathrm{~cm}^{2}$ 
geometrical area, which is an order of magnitude greater than that of MCD (0.05-5 $\left.\mu \mathrm{F} / \mathrm{cm}^{2}\right)$ [181], GC $\left(25-30 \mu \mathrm{F} / \mathrm{cm}^{2}\right)$ [181] and edge-plane graphite $\left(70 \mu \mathrm{F} / \mathrm{cm}^{2}\right)$ [213]. The geometrical area of the electrodes were calculated from the slope of the cathodic peak current (diffusion-limited current) and square root of scan rate for a $1 \mathrm{mM}$ solution of $\mathrm{K}_{3} \mathrm{Fe}(\mathrm{CN})_{6}$. (described in section 7.3.3). The high values for the specific capacitance of NCG could be due to the higher concentrations of graphite edge planes on the electrode surface. The significant presence of graphite edge planes can lead to faster kinetics and hence increased accumulation of surface charge.

As mentioned in section 7.3.2, the background current is very sensitive to capacitance, which depends upon the surface functional groups to a large extent. Figure 7.13 is the Bode plot (Section 3.5.2) obtained from EIS measurements before oxidation and after two steps of anodic oxidations. This is a plot of $\log Z$ vs $\log \omega$. The slope at lower frequencies must be -1 , while the intercept at $\omega=1$ equals to $1 / \mathrm{Cd}$.

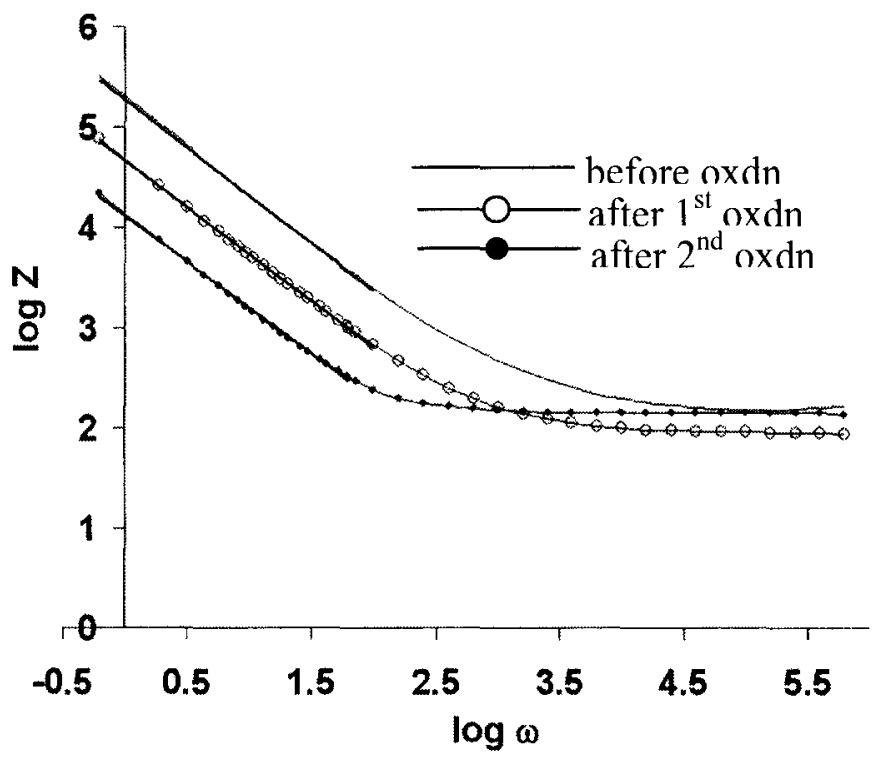

Figure 7.13 Bode plot in $0.1 \mathrm{M} \mathrm{KCl}$ solution showing the effect of anodic oxidation on the double layer capacitance of NCG electrode. 
Further, the capacitance of the material, determined using EIS, also increased dramatically from $\sim 5.5 \mu \mathrm{F}$ to $\sim 80 \mu \mathrm{F}$ for the same geometrical area $\left(0.03 \mathrm{~cm}^{2}\right)$ after two successive anodic oxidations, but did not increase significantly further. Also, successive anodic oxidations did not change the $\Delta \mathrm{Ep}$ value for dopamine redox couple. It is speculated that the electrodes displaying poor performance on initial CV experimentation had larger grain sizes or had edge planes that were bent and hence not directly exposed to the solution. Thus, they did not give a reversible response for dopamine readily. Previously, McCreery et al. showed that oxidation of the surface by electrochemical treatment creates defects on graphite [209], which may in turn increase the reactivity as noted earlier. The observed increase in capacitance and electrochemical area lend support to the hypothesis that the electrochemical anodization here creates a large number of such surface defect sites. In comparison, when MCD was anodically treated, an increase in oxygen containing functional groups on the surface was reported [216]. However, diamond surface being inert to adsorption even when terminated by oxygen containing functional groups after anodization continues to give a highly irreversible peak for the redox of dopamine $[216,217]$

\subsubsection{Stability of electrochemical response}

The NCG electrode performance, with or without electrochemical pretreatment, was reasonably stable over a large period of time. One electrode was tested with daily usage over a month and the resulting $\mathrm{CV}$ performance with dopamine is presented in Figure 7.14. As shown by Figure 7.14, the $\Delta \mathrm{Ep}$ had a negligible change over this period. The background, however, did change while maintaining the same signal to background 
ratio. This observed behavior can be explained on the basis of change in the exposed geometrical area each time the $\mathrm{CV}$ was taken. This stable behavior (of CV) is similar to that observed for MCD but contrary to the behavior of $\mathrm{GC}$, which requires frequent reactivation of the surface to overcome sluggish kinetics.

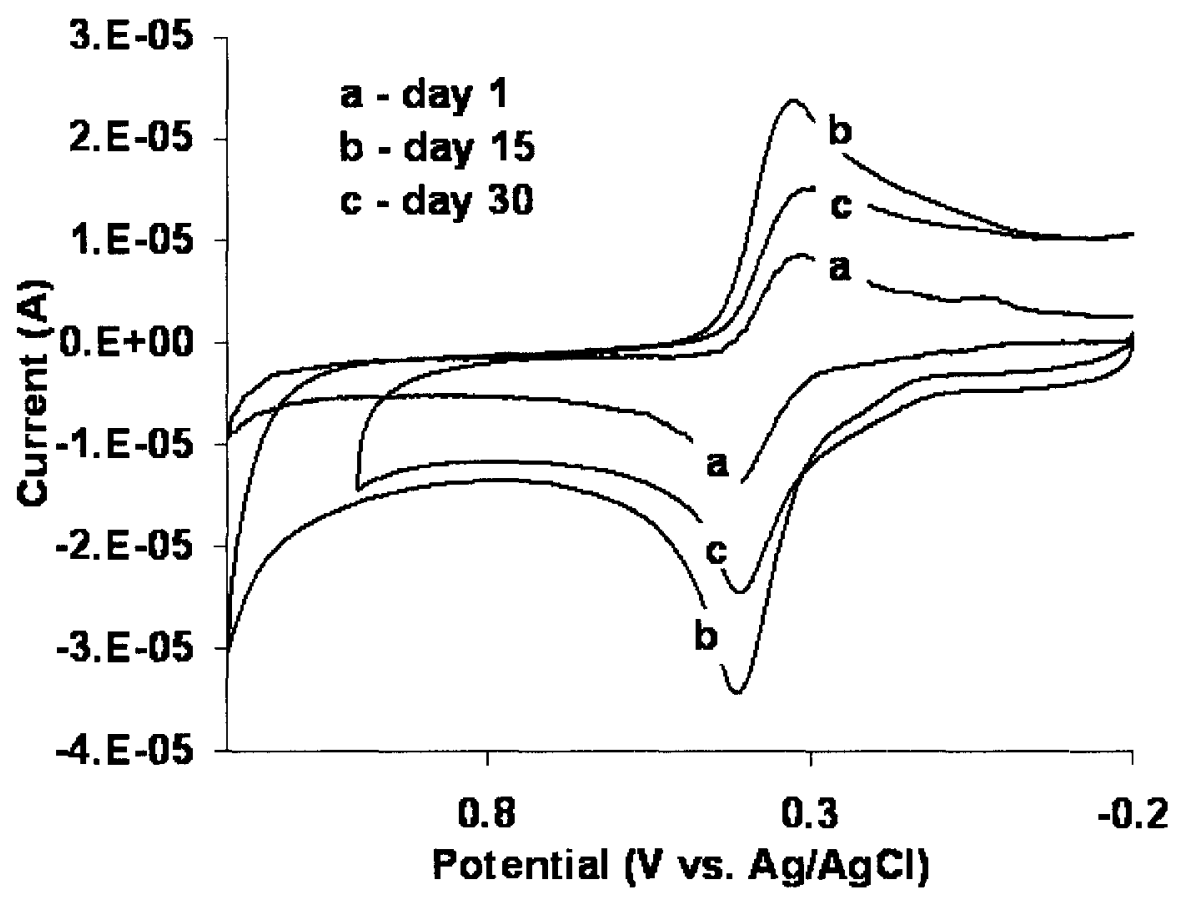

Figure $7.14 \mathrm{CV}$ of $1 \mathrm{mM}$ dopamine in $0.1 \mathrm{M} \mathrm{KCl}$ at $100 \mathrm{mV} / \mathrm{s}$ scan rate repeatedly on the same NCG electrode over a period of 1 month.

\subsubsection{Selectivity in mixtures}

The extracellular fluid in neural cells contains an excess of ascorbic acid and comparatively small amounts of dopamine, DOPAC, serotonin, etc. It is desirable to have a material that can clearly resolve the peaks for all the analytes present in a mixture. Figure 7.15 shows the $\mathrm{CV}$ obtained for a mixture of $10 \mu \mathrm{M}$ of dopamine and serotonin in 
$2 \mathrm{mM}$ ascorbic acid in $0.1 \mathrm{M} \mathrm{KCl}$. These are also the approximate ratio of concentrations in neural cells. As indicated in the plot, three peaks corresponding to ascorbic acid, dopamine and serotonin can be distinctly seen at $210,450,560 \mathrm{mV}$ respectively. The NCG electrode material exhibits reversibility for dopamine also in mixtures of analytes, where ascorbic acid and serotonin are irreversible. The result in Figure 7.15 indicates the usefulness of the NCG electrode material to detect dopamine and serotonin without interferences from ascorbic acid.

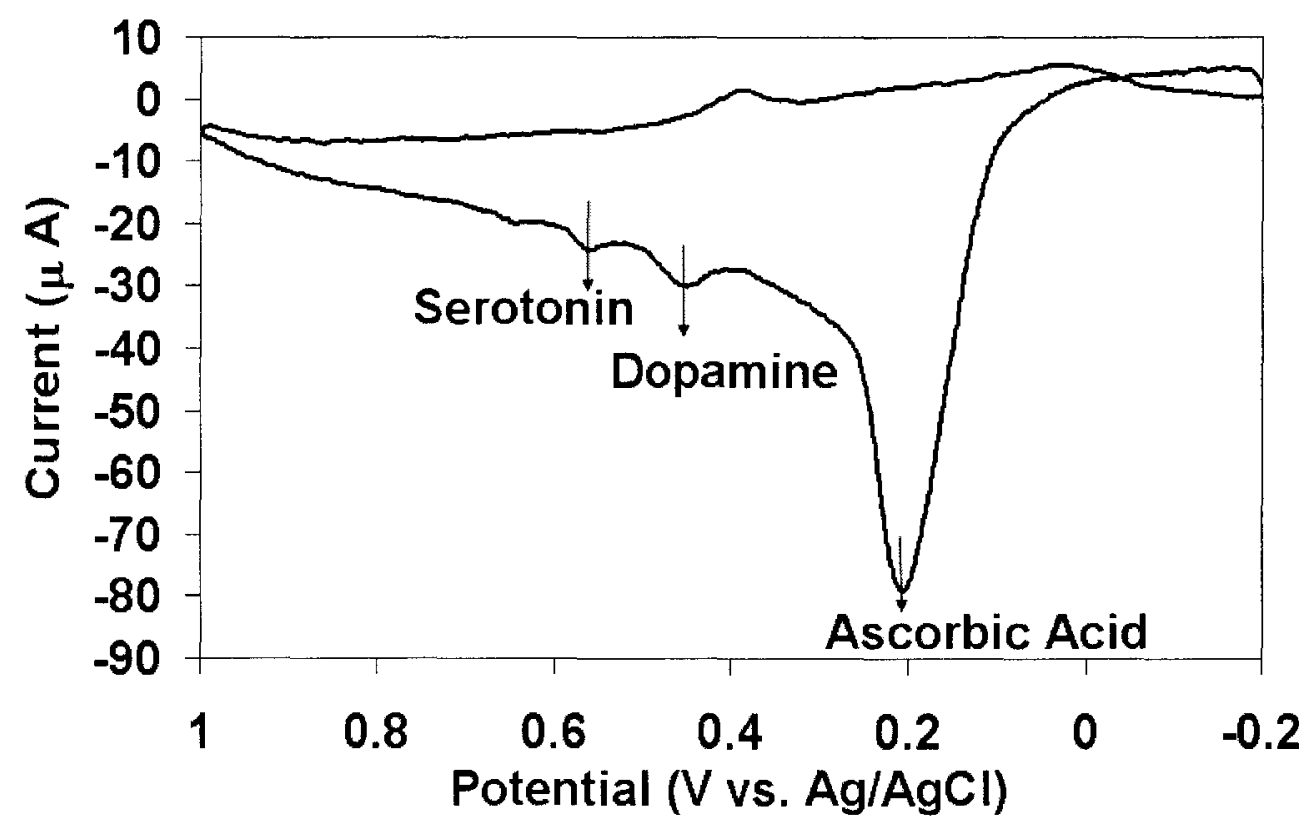

Figure $7.15 \mathrm{CV}$ of a mixture of $2 \mathrm{mM}$ of ascorbic acid, $10 \mu \mathrm{M}$ dopamine, $10 \mu \mathrm{M}$ serotonin in $0.1 \mathrm{M} \mathrm{KCl}$ solution at $100 \mathrm{mV} / \mathrm{s}$ at $\mathrm{NCG}$ electrode.

In addition to interferences with ascorbic acid and other analytes in neurological samples, the $\mathrm{pH}$ of the neurological environment is also close to 7 . The oxidation potential of dopamine is quite sensitive to $\mathrm{pH}$. At $\mathrm{pH} 7$, dopamine and ascorbic acid may oxidize at nearly the same potentials. CVs were also performed on the NCG electrodes at 
pH 7, with $10 \mathrm{mM}$ ascorbic acid and $0.1 \mathrm{mM}$ dopamine. The as-synthesized electrodes could not distinguish between ascorbic acid and dopamine oxidation at $\mathrm{pH} 7$ solutions. This was because both ascorbic acid and dopamine oxidize nearly at $\sim 200 \mathrm{mV}$ at the NCG electrode. But a single anodic treatment in $0.5 \mathrm{M} \mathrm{H}_{2} \mathrm{SO}_{4}$ reveals two distinguishable oxidation peaks for ascorbic acid and dopamine as shown in Figure 7.16 (a). This was because the anodic oxidation treatment shifted the ascorbic acid oxidation peak to lower potentials $(\sim 40 \mathrm{mV})$ but did not change the dopamine redox couple position. After about 100 continuous scans, the ascorbic acid signal in the mixture started to reduce. A similar effect has been observed for anodically oxidized graphite-epoxy electrode [218] and vacuum heat-treated GC [219]. Contrary to this result, at the surface of MCD, the oxidation peak for ascorbic acid shifts to higher potentials due to anodic pre-treatment [216].

Anodic treatment introduces oxygen functionalities at the surface as discussed previously in the XPS analysis. These oxygen functionalities presumably catalyzed the oxidation of ascorbic acid, moving its oxidation potential to lower values. As shown in Figure $7.16 \mathrm{~b}$, continuous scanning of the electrode after anodic oxidation did not change the response of ascorbic acid even after hundred scans. However, when the electrode was introduced in a mixture containing dopamine and ascorbic acid, the signal of ascorbic acid reduced to a small extent over the period of the hundred scans as shown in Figure 7.16a. 

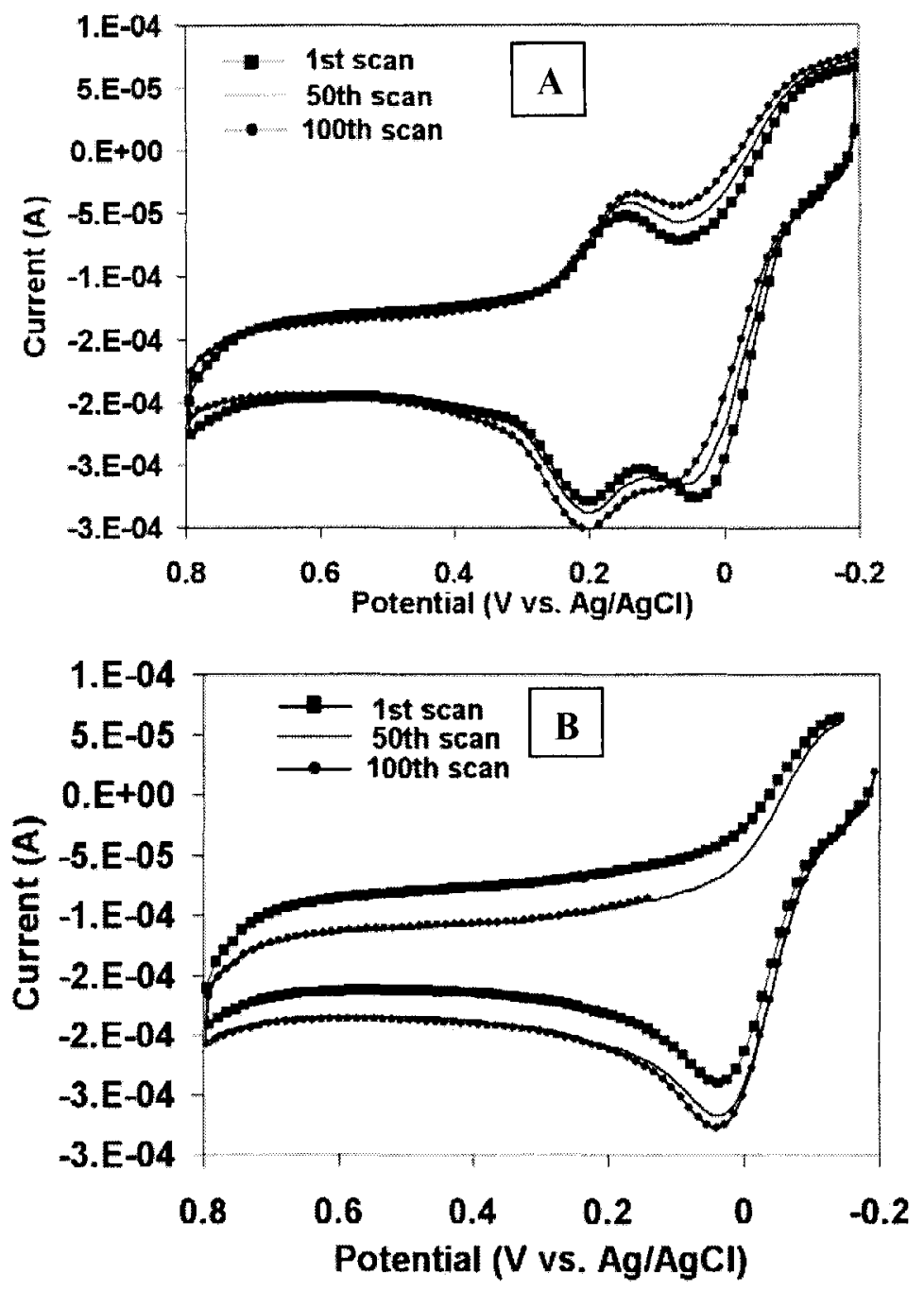

Figure 7.16 CV obtained after anodic oxidation during continuous scanning (100 scans) at $100 \mathrm{mV} / \mathrm{s}$ at $\mathrm{pH} 7$ phosphate buffer solution containing (a) mixture of $10 \mathrm{mM}$ of ascorbic acid, $0.1 \mathrm{mM}$ dopamine mixture (b) $10 \mathrm{mM}$ ascorbic acid at NCG electrode.

Dopamine is known to adsorb strongly at the surface of graphite. This adsorption is enhanced because of the increase in surface oxides with anodic treatment in acid (Section 7.3.7). It is assumed that eventually dopamine covers all the active sites available on the electrodes surface, which were initially available as oxide sites to 
catalyze ascorbate oxidation. The results in Figure 7.16 indicate the possibility of using of the NCG electrode to detect in vivo concentrations of dopamine in the presence of excess ascorbic acid.

Thus, the as-synthesized NCG electrode material exhibited high capacitance values and fast kinetics for adsorption-related electron transfer processes indicating the presence of a high density of exposed edge sites. This NCG electrode, when used as an electrochemical sensor, provides good $\mathrm{CV}$ peak-separation and response for dopamine and ascorbic acid in both $\mathrm{KCl}$ and $\mathrm{pH} 7$ buffer solutions. The only surface treatment that was needed for a few electrodes was a one-time anodic oxidation in $0.5 \mathrm{M} \mathrm{H}_{2} \mathrm{SO}_{4}$. The attractive features of this NCG material are the ease of synthesis, and the stable and reversible electrochemical response for catechols and quinones and their mixtures with others such as serotonin and ascorbic acid for extended periods of usage. The NCG material presents an interesting possibility for in vivo electroanalytical detection of neurological fluids.

\subsection{Carbon nanopipettes as electrochemical sensors}

As described in Section 6.3, carbon nanopipettes are carbon nanostructures with an outer cone made up of wrapped up sheets of graphite. They have an constant diameter hollow core, which is a single/multi-walled carbon nanotube. Hence, due to the high density of edge sites at the outer wall, they could exhibit a reversible electrochemistry similar to NCG. Also, due to their small dimensions, they can be used as a nanoelectrode. 
Irrespective of the shape of an electrode, the concentration profile (analyte solution concentration as a function of the distance from the surface of the electrode) is obtained by solving the Fick's second law, choosing the appropriate co-ordinate system. However, the concentration profiles change depending on the co-ordinate system. Taking an example of a spherical / hemispherical electrode system (e.g., just the tip of a nanotube), the Fick's law for diffusion is given by [137]:

$\frac{\partial C(r, t)}{\partial t}=D\left\{\frac{\partial^{2} C(r, t)}{\partial r^{2}}+\frac{2}{r} \frac{\partial C(r, t)}{\partial r}\right\}$

where $r$ is the radial distance from the electrode center. The boundary conditions then are:

$\mathrm{C}(\mathrm{r}, 0)=\mathrm{C}_{\mathrm{b}}\left(\mathrm{r}>\mathrm{r}_{\mathrm{o}}\right)$

$\operatorname{Lim}(r \rightarrow \infty) C(r, t)=C_{b}$

$C\left(r_{0}, t\right)=0(t>0)$, where $r_{0}$ is the radius of the electrode and $C_{b}$ is the bulk concentration of the specie. Solving equation (7.4) gives the concentration of species as a function of time and radial distance from the electrode surface $r_{0}$.

The mass flux at the electrode surface is proportional to the current, hence:

$-J\left(r_{o}, t\right)=\frac{i(t)}{n F A}=D\left[\frac{\partial C(r, t)}{\partial r}\right]_{r=r o}$

The resulting equation, after solving equation 7.4 with appropriate boundary conditions, gives the diffusion current for a spherical electrode as:

$i_{d}(t)=n F A D C_{b}\left[\frac{1}{(\pi D t)^{0.5}}+\frac{1}{r_{o}}\right]$,

which can be written as:

$\mathrm{i}_{\mathrm{d}}($ spherical $)=\mathrm{i}_{\mathrm{d}}($ linear $)+\mathrm{nFADC}_{\mathrm{b}} / \mathrm{r}_{\mathrm{o}}$ 
Comparing this equation with equation 7.2 for a planar electrode at infinite time, the diffusion current approaches zero for a planar electrode, while for a spherical electrode,

$$
\lim _{t \rightarrow \infty} i_{d}=\frac{n F A D_{o} C_{o}^{*}}{r_{o}}
$$

The reason for this non-zero current is that the surface is not able to draw enough of flux from a continually larger area at its outer limit. With ultra-microelectrodes, steady state currents can be achieved at much shorter times.

The concentration profile for electroactive species near the spherical electrode is given by [137]:

$$
C(r, t)=C_{b}\left[1-\frac{r_{o}}{r} \operatorname{erfc}\left(\frac{r-r_{o}}{2(D t)^{0.5}}\right)\right]
$$

This equation can be used to obtain the boundary layer for a given time and radius of a spherical electrode. Figure 7.17 gives the boundary layer thickness as a function of radius of an electrode for $t=0.1 \mathrm{~s}$. As seen from the figure, for small electrodes, the boundary layer thickness (estimated at $98 \%$ of $C_{b}$ ) is much greater than the radius of the electrode. Table 7.3 indicates that the $\delta / \mathrm{r}$, where $\delta$ is boundary layer thickness, becomes larger for smaller radius.

The solution of the Fick's equation in cylindrical co-ordinates is far more complicated and has a solution in terms of Bessel functions. 


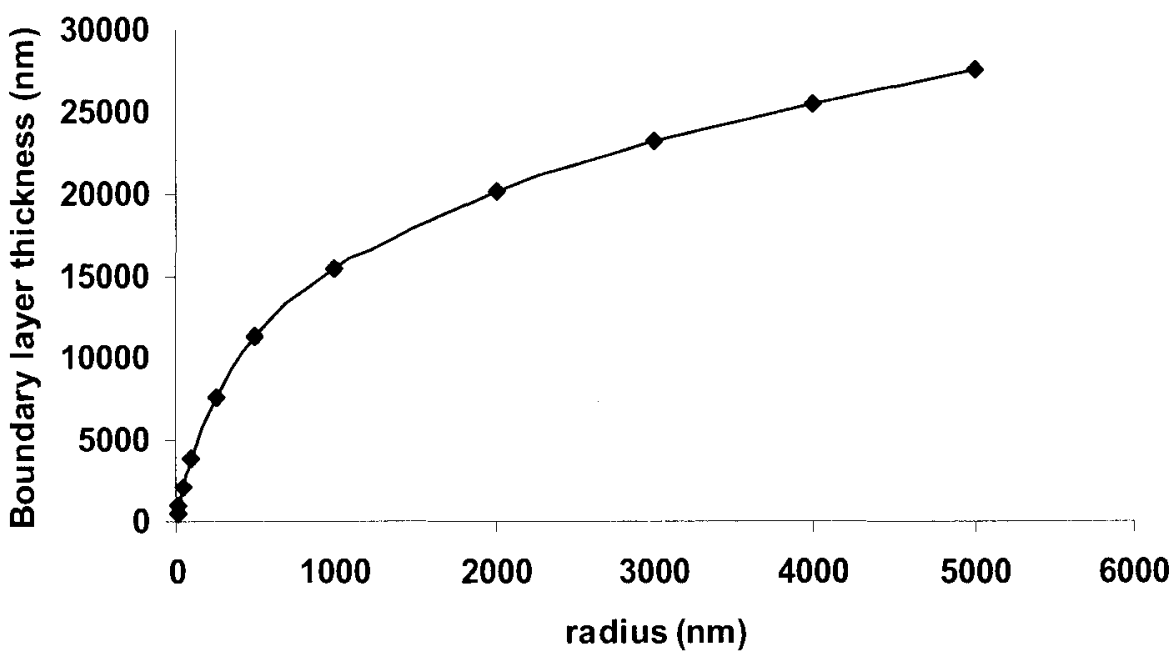

Figure 7.17 Dependence of the boundary layer thickness, $\delta$, with spherical nanoelectrode radius.

Table 7.3 Dependence of boundary layer thickness, $\delta$ (at $98 \% \mathrm{C}_{\mathrm{b}}$ ), and $\delta / \mathrm{r}$ on the nanoelectrode radius $(r)$.

\begin{tabular}{|l|l|l|}
\hline $\mathrm{rnm}$ & $\delta \mathrm{nm}\left(98 \%\right.$ of $\left.\mathrm{C}_{\mathrm{b}}\right)$ & $\delta / \mathrm{r}$ \\
\hline 10 & 520 & 52 \\
\hline 20 & 1010 & 50.5 \\
\hline 50 & 2180 & 43.6 \\
\hline 100 & 3970 & 39.7 \\
\hline 250 & 7660 & 30.6 \\
\hline 500 & 11360 & 22.7 \\
\hline 1000 & 15420 & 15.4 \\
\hline 2000 & 20060 & 10.0 \\
\hline 3000 & 23164 & 7.72 \\
\hline 4000 & 25528 & 6.38 \\
\hline 5000 & 27596 & 5.52 \\
\hline
\end{tabular}

Electrodes with at least one dimension (critical dimension) lesser than $25 \mu \mathrm{m}$ are called ultra-microelectrodes (UME), while electrodes with $<10 \mathrm{~nm}$ dimensions are called 
nanodes [137]. Since carbon nanopipettes are about $1 \mu \mathrm{m}$ at the base and about 10-20 nm at the tip, they are classified as UME.

The concentration profile and steady state currents change with change in the electrode shape. Hence, in general, for UME, irrespective of geometry, for short-times, where the diffusion layer thickness is small compared to the critical dimension, the current at any UME behaves like that for a planar electrode and follows the Cottrell equation 7.2. At long enough times, the diffusion layer thickness becomes large compared to the critical dimensions. Hence, the current at the UME approaches a steady state or a quasi-steady state. This steady state current is given by:

$\mathrm{I}_{\mathrm{ss}}=\mathrm{nFAm}_{\mathrm{o}} \mathrm{C}_{\mathrm{b}}$

where $m_{0}$ is the mass transfer coefficient, which depends on the geometry. For a sphere, $m_{0}$ is given by $D / r_{0}$, and for a cylinder $m_{0}$ is given by $\left(2 D_{0}\right) /\left(r_{0} \ln \left(4 D_{0} R T / n F v r_{o}{ }^{2}\right)\right)$. Thus, often for spherical UME, the cyclic voltammograms are sigmoidal in shape at reasonably large $\mathrm{t}$ and reach peak shaped behavior at very small $\mathrm{t}$. However, for a cylindrical microelectrode, steady state is never reached.

There are several advantages of using nanopipettes as nanoelectrodes. The small dimension of the electrode with fast kinetics helps in reducing the time scales for detection. This can be advantageous in detecting transient analytes rcleased during a complex reaction. In the case of planar electrodes, the ratio of faradaic currents $\left(i_{i}\right)$ (current due to redox reactions) and charging current $\left(i_{c}\right)$ is inversely proportional to the scan rate and double layer capacitance. Thus, as the scan rate increases, it becomes difficult to differentiate between $i_{f}$ and $i_{c}[220]$. In the case of microelectrodes operating 
at steady state, the ratio of $i_{f} / i_{c}$ becomes inversely proportional to $1 / r_{0}$. Thus, the smaller the electrode, the higher will be $\mathrm{i}_{\mathrm{f}} / \mathrm{i}_{\mathrm{c}}[220]$.

Carbon nanopipettes can be approximated as cylindrical electrodes due to their small conical angles. When the concentration profile is assumed to be constant with the length of a cylinder, the diffusion equation must be solved for cylindrical co-ordinates, which is quite complicated. For the sake of an order of estimation, the boundary layer thickness variation with radius of the pipette can be assumed to follow that of a spherical electrode shown in Figure 7.17. In order to use carbon nanopipettes as nanoelectrodes, the separation between the pipettes must be such that their diffusion boundary layers do not overlap. Typically, for a pipette diameter of $0.5 \mu \mathrm{m}$, assuming mass transfer just at the tip, the diffusion boundary layer is on the order of $4 \mu \mathrm{m}$ (from spherical/hemispherical diffusion). Thus, to show a true nanoelectrode behavior, they must be sparsely located on a substrate. This nanoelectrode can then be used for fast cyclic voltammetry (as steady state is achieved fairly quickly compared to a macroelectrode).

The carbon nanopipettes were tested for their electrochemical response in the following solutions: $1 \mathrm{mM} \mathrm{K}{ }_{3} \mathrm{Fe}(\mathrm{CN})_{6}$ in $0.1 \mathrm{M} \mathrm{KCl}$, and $1 \mathrm{mM}$ dopamine in $0.1 \mathrm{M} \mathrm{KCl}$. The first substrate tested for electrochemical response had a very small region exposed to the solution. The approximate geometrical area of this region is $10^{-4} \mathrm{~cm}^{2}$. This small region had a huge density of nanopipettes, which are not grown in any particular direction (Figure 7.18(a)). Thus, the diffusion boundary layers of the individual pipettes significantly overlapped. However, since the area of exposure was so small, the electrode in essence was behaving like a microelectrode. Accordingly, the electrochemical 
response of dopamine $(1 \mathrm{mM}$ in $0.1 \mathrm{M} \mathrm{KCl})$ as indicated in figure $7.18 \mathrm{~b}$ was sigmoidal in shape i.e. steady state was achieved. When increasing the scan rate (in Figure 7.18(c), the steady state current increased with scan rate $v(\mathrm{~V} / \mathrm{s})^{\wedge} 0.22$, unlike diffusion limited cases, where the current depends on the square root of scan rate. The currents were calculated by subtracting the background current as indicated in Fig. 7.18b, a method given in ref [196]. Increasing the scan rate beyond $1 \mathrm{~V} / \mathrm{s}$ did initiate the peak shaped response, but this was accompanied by an increase in the background current, making it more difficult to detect the signal from the analyte.
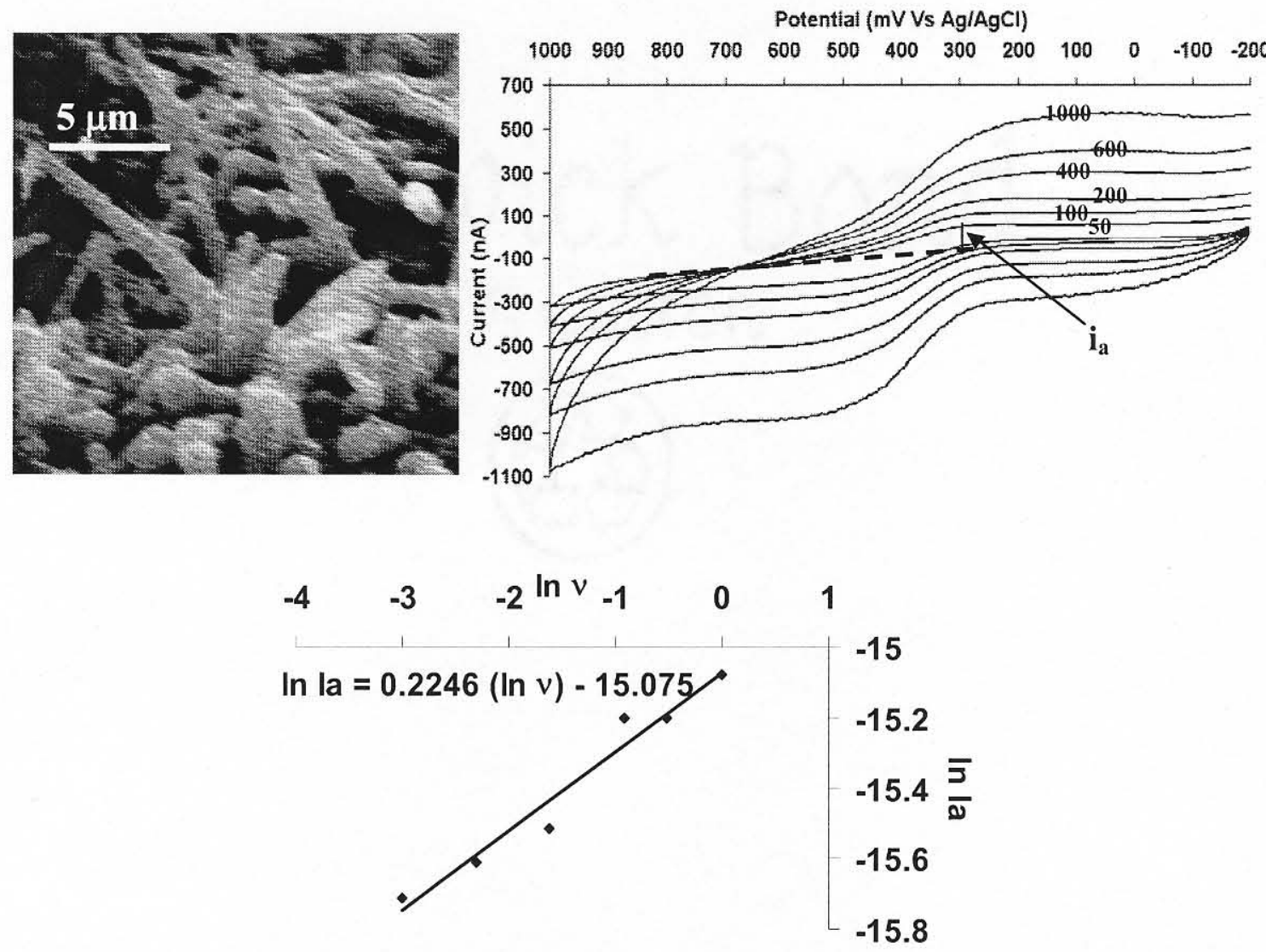

Figure 7.18 (a) SEM image of randomly oriented pipettes on a platinum wire. (b)

Steady-state response from the pipettes to a $1 \mathrm{mM}$ dopamine in $0.1 \mathrm{M} \mathrm{KCl}$. (c) Dependence of anodic current on scan rate. 
The approximate electrochemical area calculated from the response to dopamine (by Randles-Sevick equation) was very close to the geometrical area of the exposed pipette array. The reason for such a behavior could be that in the case of this randomly oriented nanopipette electrode, the area of the bunch of nanopipettes that were exposed to the solution was itself extremely small. This was making the electroactive material exposed to the solution act as a microelectrode. Thus, although the diffusion boundary layers of the nanopipettes were actually overlapping, but due to the small active area, the response was similar to a microelectrode.

In order to make use of the nanoscale of the pipettes in a nanoelectrode, one would need to have them in the form of arrays, such that the distance between them crosses the minimum needed for non-overlapping diffusion boundary layers [195]. To understand this type of a behavior, a sample in which the nanopipettes were grown for short time on a platinum-plated diamond-coated wire for 1 hour was used. The sample contained nanostructures with varying aspect ratio as described in section 6.4 Figure 6.10. Regions at the tip of the wire substrate contained a conical nanostructure with a central nanotube. As we move away from the tip, due to competing growth and etching of the surrounding graphite sheets, the aspect ratios of these nanostructures change eventually giving nanopipettes. One such an electrode was carefully coated with epoxy such that just this region of nanostructures with varying aspect ratios pipettes were exposed to the solution (Figure 7.19). 


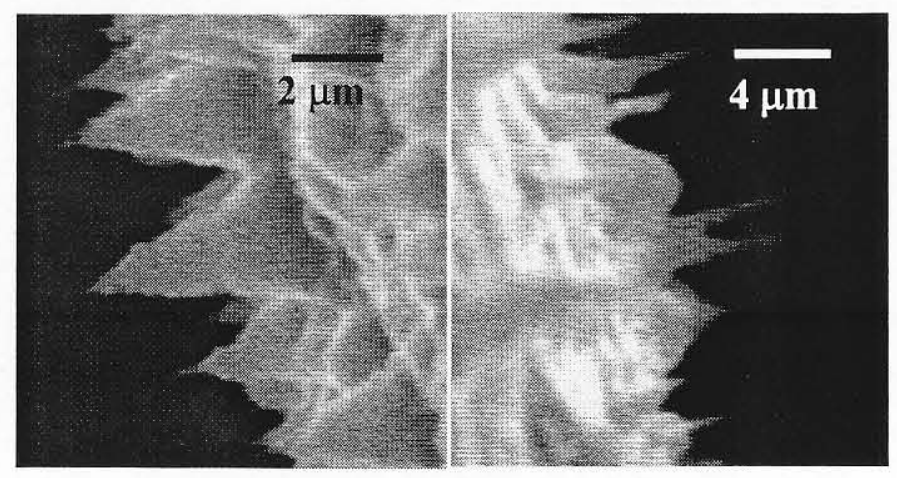

(a)

(b)

Figure 7.19 (a) SEM image of array electrode containing cones with a central nanotube.

(b) SEM image of nanopipettes about $2 \mathrm{~mm}$ away from the tip.

Figure 7.20(a) shows the response of such an electrode to $1 \mathrm{mM}$ Dopamine in $0.1 \mathrm{M} \mathrm{KCl}$ solution before and after treatment in $0.5 \mathrm{M} \mathrm{H}_{2} \mathrm{SO}_{4}$ at a scanning window of $2 \mathrm{~V}$ to $-2 \mathrm{~V}$. As seen in the figure, the peak separation $(\Delta \mathrm{Ep})$ before acid treatment was $300 \mathrm{mV}$ and after acid treatment was $100 \mathrm{mV}$. This is similar to what was observed for nanocrystals of graphite with edge planes exposed to the solution. The reaction for 1 $\mathrm{mM} \mathrm{K}_{3} \mathrm{Fe}(\mathrm{CN})_{6}$ in $0.1 \mathrm{M} \mathrm{KCl}$ was also reversible at the electrode surface and seemed to have a square root dependence with scan rate (Figure $7.20(\mathrm{~b})$ ). Thus, once again, the electrode was behaving like a macroscopic electrode. The diffusion boundary layers were overlapping, causing the electrode to have an electrochemical area equivalent to the geometric area. 


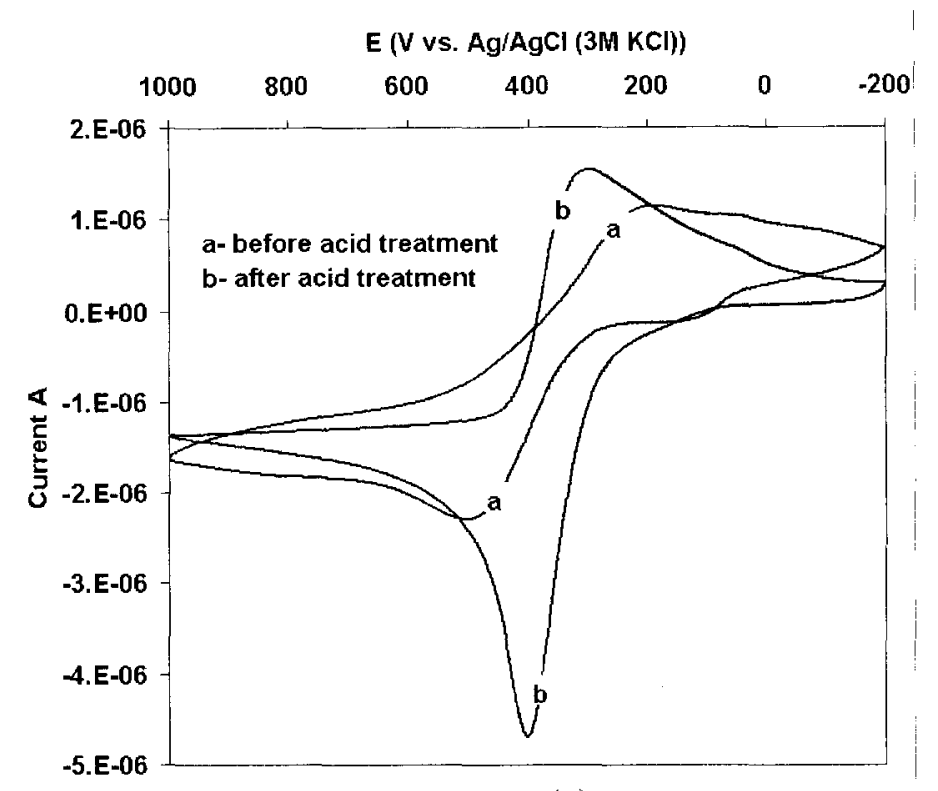

(a)

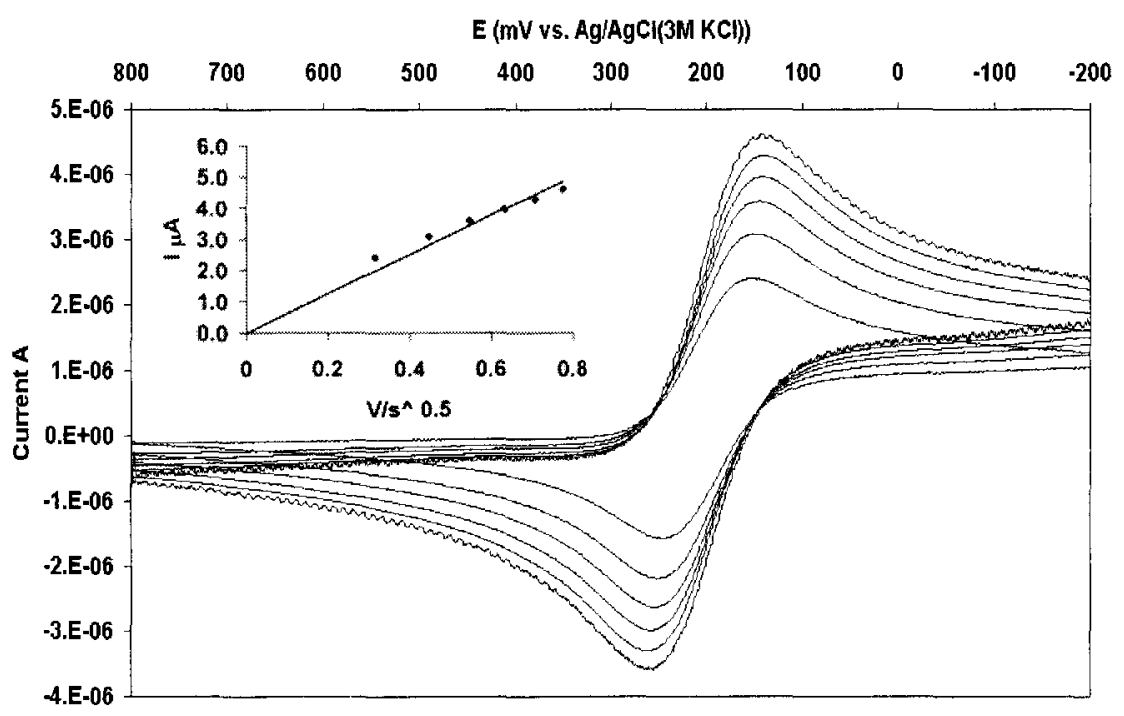

(b)

Figure 7.20 (a) $\mathrm{CV}$ of $1 \mathrm{mM}$ dopamine in $0.1 \mathrm{M} \mathrm{KCl}$ at $100 \mathrm{mV} / \mathrm{s}$ before and after electrochemical treatment in $0.5 \mathrm{M} \mathrm{H}_{2} \mathrm{SO}_{4}$ at the nanopipette array electrode. (b) $\mathrm{CV}$ of 1 $\mathrm{mM} \mathrm{K}{ }_{3} \mathrm{Fe}(\mathrm{CN})_{6}$ in $0.1 \mathrm{M} \mathrm{KCl}$ at $100 \mathrm{mV} / \mathrm{s}$ to $600 \mathrm{mV} / \mathrm{s}$. Inset shows dependence of cathodic current with scan rate $(\mathrm{V} / \mathrm{s})^{\wedge} 0.5$. 
The area calculated again from this experiment was approximately equal to the geometrical area. This indicates that even though the pipettes are in an array form, the distance between each individual pipette was smaller than the boundary layer thickness. However, it seems certain from Figures 7.18 and 7.20 that the nanopipettes are electrochemically active.

\subsection{Nanoarray sensor}

The distance between the pipettes could be further increased to get the response from each individual pipette. A very simple technique could be used for this by taking advantage of the conical morphology of the nanopipettes. The nanopipette array in a sample can be coated with a polymer such that only the top few tens of nm are exposed to the solution (in effectively, one would utilize the conical geometry of the nanopipettes to engineer the spacing between the exposed nanopipette tips). The diameter of the tip of the pipettes are only about $10-20 \mathrm{~nm}$, while their base is about $1000 \mathrm{~nm}$, hence, automatically, the tips of the pipettes are well spaced in the array (See Fig. 7.21). 


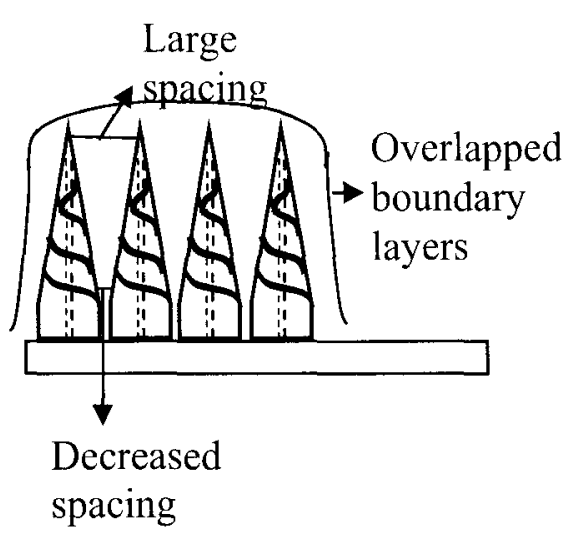

(a)

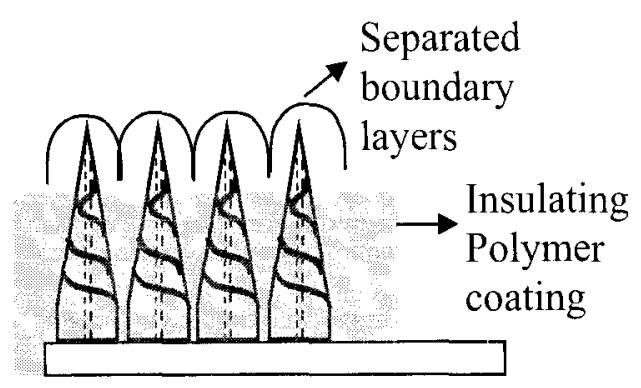

(b)

Figure 7.21 Schematic of a closely spaced array of nanopipettes (a) without coating and (b) with polymer coating.

Figure 7.22 shows such an array of nanopipettes dip-coated with an insulating enamel. However, successful electrochemical measurements from these pipette arrays have not yet been achieved due to some practical problems of adherence of the enamel to the substrate. Using a polymer as an insulating layer between the pipettes may be a better solution due to the uniformity of coating achievable and control in the thickness of the coating. 

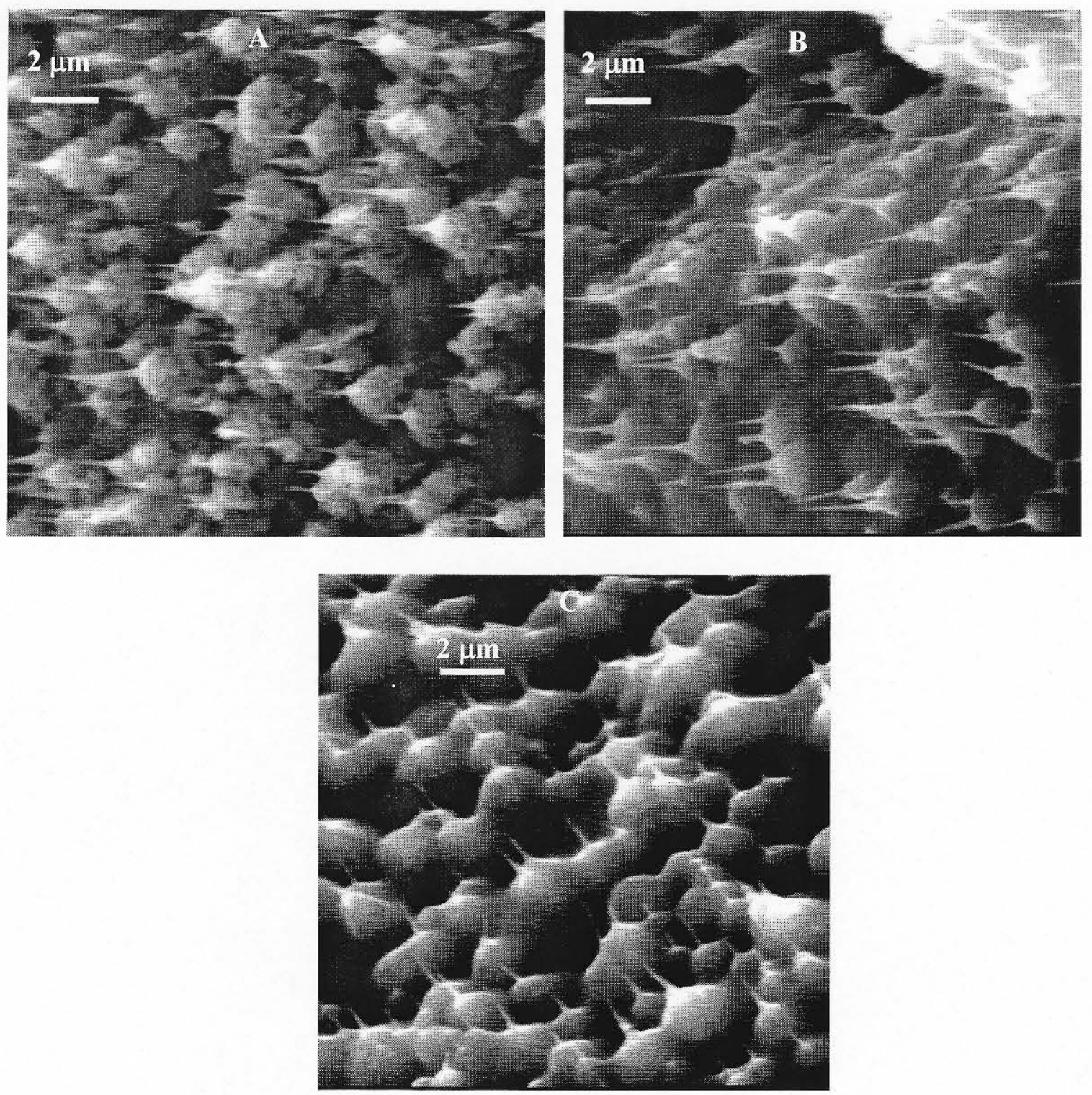

Figure 7.22 SEM images of (a) an uncoated array of nanopipettes, sequential coating of the array with enamel (b) after $1^{\text {st }}$ dip-coating, and (c) after second dip-coating.

Heat treatment to remove the impurities associated with multi-walled carbon nanotubes such as amorphous carbon, graphitic particles and catalyst particles increases the peak separation for $\mathrm{K}_{3} \mathrm{Fe}(\mathrm{CN})_{6}$ analyte from $100 \mathrm{mV}$ to $230 \mathrm{mV}$ with an increase in 
the volume specific capacitance [196]. This was attributed to the removal of impurities exposing only the basal planes of graphite, which is known for its slow electron transfer kinetics. Hence, one cannot really make use of the longitudinal area of carbon nanotubes for sensing because they are nearly inert. Thus, even when used as a paste electrode, carbon nanotubes rely on defects at their basal planes or the amorphous and graphitic particles for electrochemical response.

To use carbon nanotubes as nanoelectrode arrays, they were grown on patterned silicon substrates, such that there were specific regions of the nanotube-bundles on the substrate [196]. The space between the nanotube bundles were covered by an insulating material, such that only the tips of the nanotube bundles were exposed. The nanotube bundles were separated sufficiently from each other such that they had non-interfering boundary layers. This type of a fabrication of a sensor requires several expensive steps of microfabrication. In comparison, carbon nanopipettes array sensors discussed above would require a simple and cheap process of dip coating in a polymer and curing.

When used individually as a single nanoelectrode, carbon nanopipettes are expected to be more electrochemically active due to their reactive edge sites along their length, compared to carbon nanotubes, which have comparatively inert basal planes. Thus carbon nanopipettes have shown a promise as an interesting electrode material. 


\subsection{Summary}

NCG films synthesized by CVD exhibited high capacitance values and fast kinetics for adsorption-related electron transfer processes indicating the presence of a high density of exposed edge sites. This NCG electrode, when used as an electrochemical sensor, provides good $\mathrm{CV}$ peak-separation and response for dopamine and ascorbic acid in both $\mathrm{KCl}$ and $\mathrm{pH} 7$ buffer solutions. 'The only surface treatment that was needed for a few electrodes was a one-time anodic oxidation in $0.5 \mathrm{M} \mathrm{H}_{2} \mathrm{SO}_{4}$. The attractive features of this NCG material are the ease of synthesis, and the stable and reversible electrochemical response for catechols and quinones and their mixtures with others such as serotonin and ascorbic acid for extended periods of usage. The NCG material presents an interesting possibility for electroanalytical detection of neurological fluids in vivo.

Carbon nanopipettes on the other hand have a similar edge-site dominated structure, because of which they gave a similar electrochemical response like NCG electrodes. However, to utilize their potential as nanoelectrode sensors, arrays of nanopipettes must be covered by insulating polymers such that only their tips are exposed to the solution and their diffusion boundary layers do not overlap. Such an array sensor is easy to synthesize because of the geometry of the nanopipette. Even when used as individual nanodes, carbon nanopipettes would be superior to carbon nanotubes because of their highly reactive edge-plane sites, compared to carbon nanotubes with inert basalplane sites. 


\section{CHAPTER 8}

\section{CONCLUSIONS}

Nucleation experiments were carried out to understand the path for diamond nuclei formation and the structure of the intermediate phases to diamond nucleation. Early stages of growth experiments were carried out to understand the kinetic mechanisms that control the morphology of diamond and graphite crystals.

To study nucleation, large densities of carbon nanocrystals were synthesized on platinum wire substrates and their structure was extensively analyzed using electron nanodiffraction and theoretical simulations. The main conclusions from these studies are summarized below:

- Most of the non-graphitic nanocrystals $(\sim 2-5 \mathrm{~nm})$ had crystal structure different from cubic-diamond. They were identified as hexagonal diamond (lonsdaleite), fcc-carbon or bcc-carbon. Bcc-carbon was a new phase discovered in these experiments.

- Simulations of electron diffraction patterns using Mac Tempas multislice method from clusters of carbon atoms clearly eliminated the possibility of stacking faults, vacancies, or mixed $\mathrm{sp}^{2}$ and $\mathrm{sp}^{3}$ phases as being responsible for observing forbidden spots corresponding to the fcc-carbon or bcc-carbon phase. 
- Using intensity ratios of electron diffraction patterns and calculated lattice parameters, the structures of fcc and bcc phases of carbon were hypothesized as possibly $\mathrm{CH}_{\mathrm{x}}$ phases with hydrogen incorporation within the carbon lattice.

- Energy calculations (DFT) predicted a metastable phase of $\mathrm{CH}$ structure with lattice parameter of $0.358 \mathrm{~nm}$, which matches the indexing and intensity ratios of electron diffraction patterns for fcc carbon. The new bec phase discovered was explained possibly as a $\mathrm{CH}_{2}$ structure with a lattice parameter of $0.31 \mathrm{~nm}$, which matched the experimentally determined electron diffraction patterns.

- The metastable fcc and bcc phases $\left(\mathrm{CH}_{\mathrm{x}}\right.$ phases) support previous theories on the possible role of hydrogen in diamond nucleation.

- Nanocrystals of graphite synthesized were similar to bulk graphite in structure, but the surface sites seemed to have large edge-site density, as confirmed by electroanalytical surface chemistry.

Early stages of growth studies for diamond were carried out using interrupted growth experiments and Kinetic Monte Carlo simulations. The main conclusions are as follows:

- Experimental evidence for the change in morphology of a decahedral crystal to an icosahedral crystal was observed during growth, which supported previous results from theoretical studies.

- Using KMC simulations it was found that the slow step-propagation of $\{111\}$ and fast kink propagation at $\{100\}$ sites lead to the formation of star-shaped crystals, characterized by dimples and additional $\{111\}$ facets. The same mechanism 
gives rise to dimples in an icosahedral crystal, which are commonly observed experimentally.

- Fast step-propagation of $\{111\}$ type sites, along with relatively fast kink propagation on $\{100\}$ sites give rise to decahedral crystals.

A novel morphological manifestation of carbon nanotube called carbon nanopipette were also synthesized. Carbon nanopipettes are made up of a central, uniform diameter, hollow core of 10-20 nm, with an outer conical shape. They are thus open-ended structures, with a base of a micron, and tip of 10-20 nm, and controllable lengths from $10 \mu \mathrm{m}$ to about $75 \mu \mathrm{m}$. Growth mechanism studies for carbon nanopipettes can be summarized as below:

- Carbon nanopipette growth begins with a central carbon nanotube, and because of simultaneous growth and etching of the surrounding helical sheets of graphite, they develop a conical morphology.

- Depending on the competition between growth and etching, their aspect ratios can be controlled. Successful growth of nanopipettes in the form of arrays was accomplished by using thin films of platinum.

Surface electrochemical characterization for nanocrystalline graphite and carbon nanopipettes resulted in the following conclusions:

- Nanocrystalline graphite and carbon nanopipettes were both dominated by edge planes of graphite, due to which they exhibited reversible and stable electrochemical response for adsorption-related catechol and quinone electron transfer reactions. 
- Nanocrystalline graphite also showed good sensitivity for an important biological compound, dopamine, in a mixture of neurological solutes at neural cell concentrations, demonstrating its importance as an in vivo sensor.

- By taking advantage of their conical geometry, nanoelectrode arrays could be easily fabricated out of a synthesized array of carbon nanopipettes using a very simple method of dip-coating. Nanoelectrode arrays of carbon nanopipettes could be used for fast cyclic voltammetry. 


\section{CHAPTER 9}

\section{FUTURE SCOPE}

The outcomes from this dissertation will directly impact three broad areas: the discovery of new phases of carbon, modeling the morphological evolution of crystals and nanostructures using Kinetic Monte Carlo (KMC) simulations, and fluid sensing/delivery applications using novel nanostructures such as carbon nanopipettes.

\subsection{New phases of carbon}

Metastable phases of carbon with structures different from the known phases of diamond and graphite were discovered at the nanoscale. On the basis of lattice parameter, intensity ratios in diffraction patterns, and energy calculations, it is suggested that there may be hydrogen incorporation in the carbon lattices. This involves some unprecedented bonding for carbon and hydrogen, not known till now to exist. A direct evidence of hydrogen incorporation in the carbon crystal lattice would be desirable. If the newly discovered fcc and bcc phases of carbon could be synthesized in a nanocrystalline film form, then one could use neutron diffraction or electron recoil detection analysis to selectively detect hydrogen within the structure of these phases.

The knowledge on the bonding state of carbon and hydrogen would also be of a lot of interest. Also, if such a structure of carbon and hydrogen were possible, a $\mathrm{CH}_{\mathrm{x}}$ 
compound would be very useful as a light hydrogen storage material, and may be explored for fuel cell applications.

\subsection{KMC simulations for other materials}

KMC studies carried out as a part of this dissertation was successful in explaining the reasons for the appearance of dimples and additional faceting for star-shaped decahedral crystals and icosahedral crystals. The star-shaped crystals are also observed in other crystal systems like hexagonal boron nitride, gallium nitride, gibbsite as well as fcc gold, silver etc. (See Figure 9.1). It would be very interesting to apply these simulations to other covalent solids like gallium nitride and boron nitride, which have similar tetrahedral bonding.

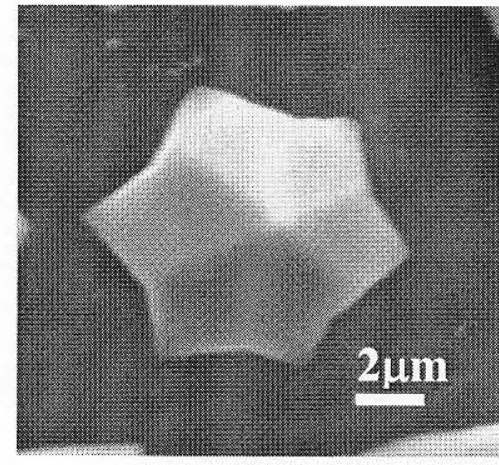

(a)

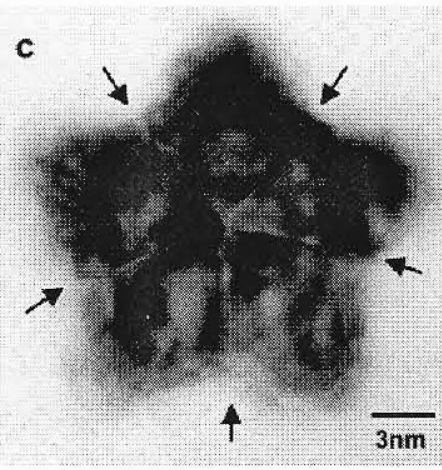

(b)

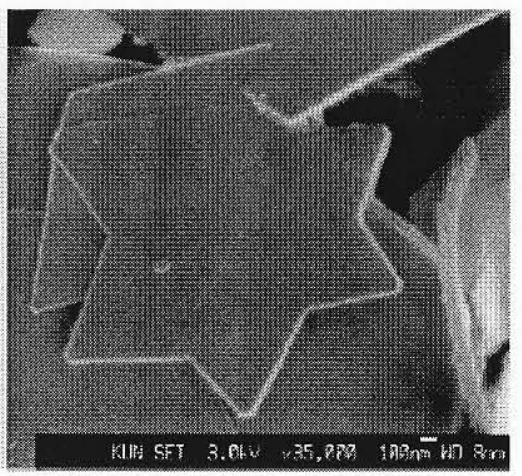

(c)

Figure 9.1 SEM images of star-shaped morphologies in other crystalline materials like (a) gallium nitride (cubic or hexagonal crystal) [courtesy H. Li], (b) hexagonal boron nitride [221], and (c) hexagonal gibbsite crystals [222]. 
Recently, there has been an unprecedented interest in one-dimensional structures of various inorganic materials. The KMC simulations described in this work could be valuable for studying kinetic faceting of nanowires/nanotubes. Particularly, predictive modeling on the morphology of nanowires based on their growth direction could help in determining their properties and help in understanding their growth processes. For instance, it would be interesting to understand kinetic faceting of carbon nanopipettes with the goal to determine the necessary changes in growth conditions leading to higher aspect ratio pipettes.

\subsection{Smart drug delivery systems using carbon nanopipettes}

Nanocrystalline graphitic phases discovered as a part of this work (nanocrystals and nanopipettes) are both useful for in vivo sensing of neurological fluids. Nanopipettes were shown to be desirable as nanoelectrodes. The other advantage apart from electroanalytical sensing is drug delivery applications. These nanostructures can be synthesized in the form of arrays and when transferred onto polymer patches, they would be ideal for transbarrier fluid delivery due to their sharp tips and hollow internal channels. Research has shown that microscopic needles when pierced through the skin do not cause pain and increase the skin permeability four folds [223]. Using these nanoscale syringes, one can not only improve the permeability, but also permit the deliver drugs at a desired rate through the hollow channels. To achieve fluid flow through these nanochannels, one would have to come up with a pressure inducing mechanism. Studying fluid flow through these nanochannels would be a challenge by itself. The patch of nanopipettes would be attached to an electronic circuitry that can 
create pressure to flow the fluid out of the pipette in the form of pulses. One could think of smart-nanobio systems, which can sense the drug level in vivo and simultaneously deliver fluids through these pipettes depending on the need (see Figure 9.2).

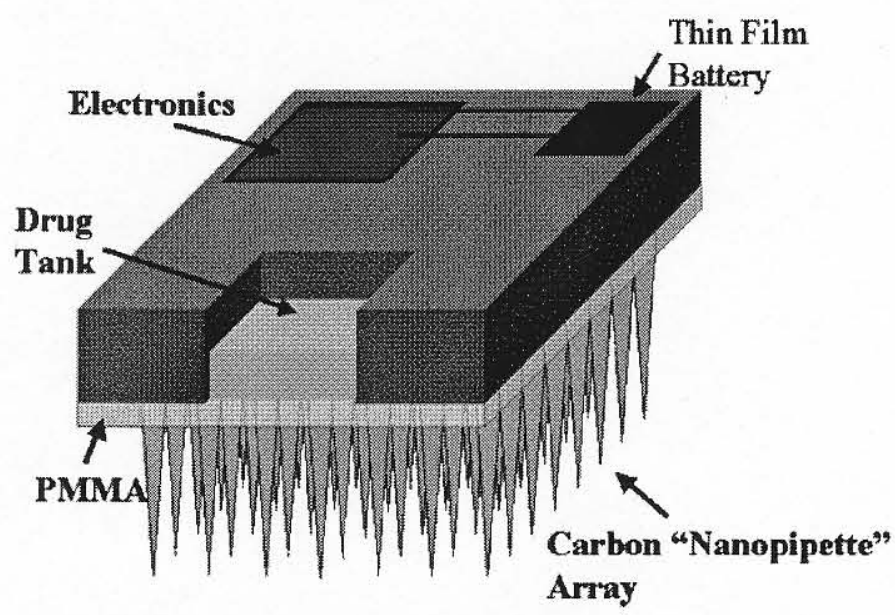

Figure 9.2 Schematic of the proposed smart drug-delivery patch using an array of carbon nanopipettes. 


\section{REFERENCES}

1. Kroto, H. W., Heath, J. R., O'Brien, S.C., Curl, R. F., \& Smalley, R. E. (1985) C60 Buckminster Fullerene. Nature 318 (6042), 162-163.

2. Bundy, F.P. (1980). The P,T phase and reaction diagram for elemental carbon, 1979. Journal of Geophysical Research 85 (NB12), 6930-6936.

3. Bundy, F. P., Bassett, W. A., Weathers, M. S., Hemley, R. J., Mao, H. K., \& Goncharov, A. F. (1996). The pressure-temperature phase and transformation diagram for carbon; Updated through 1994. Carbon 34(2), 141-153.

4. Bundy, F. P., Hall, H. T., Strong, H. M., \& Wentorf, R. H. (1955). Man-made diamonds. Nature 176, 51-55.

5. Cowan, G., Dunnington, B., \& Holtzman, A. (1968). Process for Synthesizing Diamond, U.S. Patent 3,401,019.

6. Derjaguin, B. V., Fedoseev, D. V., Spitzyn, B. V., Lukyanovich, D. V., Ryabov, B. V., \& Lavrentev, A. V. (1968). Filamentary diamond crystals. Journal of Crystal Growth 2(6), 380-384.

7. Angus, J. C., Will, H. A., \& Stanko, W. S. (1968). Growth of diamond seed crystals by vapor deposition. Journal of Applied Physics 39(6), 2915-2922.

8. Eversole, W. G., Synthesis of Diamond, U.S. Patents 3,030,187, and 3,030,188 (April 17, 1962) 
9. Stoner, B. R., Sahaida, S. R., Bade, J. P., Southworth, P., \& Ellis, P. J. (1993). Highly oriented, textured diamond films on silicon via bias-enhanced nucleation and textured growth. Journal of Materials Research 8(6), 1334-1340.

10. Gruen, D. M. (1999). Nanocrystalline diamond films. Annual Review of Materials Science 29, 211-259.

11. Bhattacharyya, S., Auciello, O., Birrell, J., Carlisle, J. A., Curtiss, L. A., Goyette, A. N., Gruen, D. M., Krauss, A. R., Schlueter, J., Sumant, A., Zapol, P. (2001). Synthesis and characterization of highly-conducting nitrogen-doped ultrananocrystalline diamond films. Applied Physics Letters 79(10), 1441-1443.

12. Lee, S. T. \& Lifshitz T. (2003). The road to diamond wafers. Nature, 424(6948), $500-501$.

13. Schreck, M., Roll, H., \& Stritzker, B. (1999). Diamond/Ir/SrTiO 3 : A material combination for improved heteroepitaxial diamond films. Applied Physics Letters $74(5), 650-652$.

14. Wang, W., Moses, T., Linares, R. C., Shigley, J. E., Hall, M., \& Butler, J. E. (2003). Gem-quality synthetic diamonds grown by a chemical vapor deposition (CVD) method. Gems and Gemology 39(4), 268-283.

15. Linares, R. C. \& Doering P. J. (2003) System and method for producing synthetic Diamond, U. S. Patent 6582513.

16. Whittaker, A. G. (1978) Carbon: A new view of its high-temperature behavior. Science 200, 763-764. 
17. Weissmantel, C., Bewilogua, K., Dietrich, D., Erler, H. J., Hinneberg, H. J., Klose, S., Nowick, W., \& Reisse, G. (1980). Structure and properties of quasiamorphous films prepared by ion beam techniques. Thin solid films 72(1), 19-31.

18. Vora, H. \& Moravec, T. J. (1981) Structural investigation of thin-films of diamondlike carbon. Journal of Applied Physics 52(10), 6151-6157.

19. Hirai, H. \& Kondo, K. (1991) Modified phases of diamond formed under shock compression and rapid quenching Science 253(5021), 772-774.

20. Endo, S., Idani, N., Oshima, R., Takano, K. \& Wakatsuki, M. (1994). X-Ray diffraction and transmission-electron microscopy of natural polycrystalline graphite recovered from high pressure Physical Review B. 49(1), 22-27.

21. Gogotsi, Y., Weiz, S., Ersoy, D. A., \& McNallan, M. J., (2001) Conversion of silicon carbide to crystalline diamond-structured carbon at ambient pressure Nature 411(6835), 283-286.

22. Lian, G. D., Dickey, E. C., Ueno, M. \& Sunkara, M. K. (2002), Ru-doped naostructured carbon films Diamond and Related Materials 11(12), 1890-1896.

23. Peng, J. L., Bursill, L. A., Jiang, B., Orwa, J. O., \& Prawer, S. (2001) Growth of c-diamond, n-diamond, and i-carbon nanophases in carbon-ion-implanted fused quartz. Philosophical Magazine B-Physics of Condensed Matter Statistical Mechanics Electronic Optical and Magnetic Properties 81 (12), 2017-2087.

24. Konyashin, I., Zern, A., Mayer, J., Aldinger, F., Babaev, V., Khvostov, V., \& Guseva, M. (2001) A new carbon modification: 'n-diamond' or face-centered cubic carbon? Diamond and Related Materials 10(1), 99-102. 
25. Fatow, M. Konyashin, I., Babaev, V., Guseva, M., Khvostov, V. \& Savtchenko, N. (2002). Carbon Modification with the fcc crystal structure Vacuum 68(1), 75 78.

26. Aust, R. B. \& Drickamer, H. G. (1963) Carbon: a new crystalline phase. Science $140(3568), 817-819$.

27. Mikiyoshida, M., Rendon, L., \& Joseyacaman, M. (1993). Cubic phases of carbon obtained from soot. Carbon 31(5), 843-846.

28. Yamada, K. (2003). Shock synthesis of a new cubic form of carbon Carbon 41(6), $1309-1313$.

29. Andersson, O. E., Prasad, B. L. V., Sato, H., Enoki, T., Hishiyama, Y., Kaburagi, Y., Yoshikawa, M., \& Bandow, S. (1998). Structure and electronic properties of graphite nanoparticles. Physical Review B 58 (24), 16387-16395.

30. Wild, C., Kohl, R., Herres, N., Mullersebert, W., \& Koidl, P. (1994) Oriented CVD diamond films: twin formation, structure and morphology Diamond and Related Materials 3(4-6), 373-381.

31. Liu, H. \& Dandy D. (1995). Diamond chemical vapor deposition: Nucleation and early growth stages, New Jersey: NY, Noyes.

32. Ferro, S. (2002). Synthesis of diamond. Journal of Materials Chemistry 12(10), 2843-2855.

33. Davis, R. F. (1993) Diamond films and coatings New Jersey, NY: Noyes.

34. Kalish, R. and Saguy, C. (2004). Electrical evaluation of defects and dopants in diamond. New Diamond and Frontier Carbon 14(2), 109-128. 
35. Pierson, H. O. (1993). Handbook of Carbon, Graphite, Diamond and fullerenesproperties, processing and applications. New Jersey, NY: Noyes.

36. Rao, A. M. \& Dresselhaus, M. S. (2001). Nanostructured carbon for advanced applications. Dordrecht: Kluwer Academic Publishsers.

37. Dresselhaus, M. S. \& Dai, H. (2004). Carbon nanotubes: Continued innovations and challenges. MRS Bulletin 29 (4), 237-239.

38. Derjaguin, B. V., Fedoseev, D. V., Lukyanovich, V. M., Spitzin, B. V., Ryabov, V. A., \& Lavrentyev, A. V. (1968). Filamentary diamond crystals. Journal of Crystal Growth 2 (6), 380-384.

39. Poferl, D. J., Gardner, N. C., \& Angus, J. C. (1973). Growth of boron doped diamond seed crystals by vapor-deposition. Journal of Applied Physics 44(4), $1428-1434$.

40. Railkar, T. A., Kang, W. P., Windischmann, H., Malshe, A. P., Naseem, H. A., Davidson, J. L., \& Brown, W. D. (2000) A Critical Review of Chemical VaporDeposited Diamond for Electronic Applications. Critical Reviews in Solid State and Materials Sciences 25(3), 163-277.

41. Ashfold, M. N. R., May, P. W., Rego, C. A., \& Everitt, N. M. (1994) Thin Film Diamond by Chemical Vapor Deposition Methods. Chemical Society Reviews 23(1), 21-30.

42. May, P. W. (2000). Diamond thin films: a $21^{\text {st }}$ centaury material. Philosophical Transactions of the Royal Society of London Series A 358(1766), 473-495. 
43. Lee, S. T., Lin, Z., \& Jiang, X. (1999) CVD diamond films: nucleation and growth. Materials Science and Engineering Reports A: A Review Journal 25(4), 123-154.

44. Bachmann, P.K., Leers, D., \& Lydtin, H. (1991). Towards a general concept of diamond chemical vapor deposition. Diamond and Related Materials 1(1), 1-12.

45. Eaton, S.C. \& Sunkara, M. K. (2000). Construction of a new C-H-O ternary diagram for diamond deposition from the vapor phase. Diamond and Related Materials 9(7), 1320-1326.

46. Bhimarasetti, G. \& Sunkara, M. K. (2003). The process window for diamond deposition from the vapor phase with sulfur in the $\mathrm{C}-\mathrm{H}-\mathrm{O}$ feed gas mixtures. Thin Solid Films 440(1-2), 78-86.

47. Mahalingam, P., Liu, H., \& Dandy, D. (1997). A model for the nucleation of diamond clusters on Si(111) substrates. Journal of Applied Physics 81(4), 19661977.

48. Matsumoto, S. \& Matsui, Y. (1983). Electron-microscopic observation of diamond particles grown from the vapor-phase. Journal of Materials Science $18(6), 1785-1793$.

49. Stein, S. E. (1990). Diamond and Graphite precursors Nature, 346(6284) 517-517.

50. Frenklach, M., Kematick, R., Huang, D., Howard, W., Spear, K. E. Phelps, A. W., Koba, R. (1989). Homogenous nucleation of diamond powder. Journal of Applied Physics 66(1), 395-399.

51. Badziag, P., Verwoerd, W. S., Ellis, W. P., \& Greiner, N. R. (1990). Nanometersized diamonds are more stable than graphite. Nature, 343(6255), 244-245. 
52. Lambrecht, W. R. L., Lee, C. H., Segall, B., Angus, J. C., Li, Z. D., \& Sunkara, M. (1993) Diamond nucleation by hydrogenation of edges of graphitic precursors. Nature 364(6438), 607-610.

53. Lee, S. T., Peng, H. Y., Zhou, X. T., Wang, N., Lee, C. S., Bello, I., \& Lifshitz, Y. (2000). A nucleation site and mechanism leading to epitaxial growth of diamond films. Science 287(5450), 104-106.

54. Sawada, H., Ichinose, H., Watanabe, H., Takeuchi, D., \& Okushi, H. (2001) Cross-sectional TEM study of unepitaxial crystallites in a homoepitaxial diamond film, Diamond and Related Materials 10, 2030-2034.

55. Lifshitz, Y., Kohler, T., Frauenheim, T., Guzmann, I., Hoffman, A., Zhang, R. Q., Zhou, X. T., \& Lee, S.T. (2002). The mechanism of diamond nucleation from energetic species. Science 297(5586), 1531-1533.

56. Bundy, F. P. \& Kasper, J. S. (1967) Hexagonal diamond - A new form of carbon. Journal of Chemical Physics 46, 3437-3446.

57. Maruyama, K., Makino, M., Kikukawa, N., \& Shiraishi, M. (1992). Synthesis of hexagonal diamond in a hydrogen plasma jet. Journal of Materials Science Letters 11, 116-118.

58. Pickard, C. J., Milman, V., \& Winkler, B. (2001) Is there theoretical evidence for a metallic carbon polymorph with space group symmetry $\mathrm{Fm} \overline{3} \mathrm{~m}$ at ambient conditions? Diamond and Related Materials 10(12), 2225-2227.

59. Murrieta, G., Tapia, A., \& de Coss, R. (2004) Structural stability of carbon in the face-centered-cubic ( $\mathrm{Fm} \overline{3} \mathrm{~m})$ phase Carbon 42(4), 771-774. 
60. Wang, S. Q. \& Ye, H. Q. (2003) First-principles study on the lonsdaleite phases of C, Si and Ge Journal of Physics- Condensed Matter 15(12), L197-L202.

61. Tsuda, M., Nakajima, M., \& Oikawa, S. (1986). Epitaxial growth mechanism of diamond crystal in $\mathrm{CH}_{4}-\mathrm{H}_{2}$ plasma, Journal of the American Chemical Society, 108(19), 5780-5783.

62. Mehandru, S. P. \& Anderson, A. B. (1990). Adsorption and bonding of $\mathrm{C}_{1} \mathrm{H}_{\mathrm{x}}$ and $\mathrm{C}_{2} \mathrm{H}_{\mathrm{y}}$ on unreconstructed diamond $\{111\}$ - dependence on coverage and coadsorbed hydrogen. Journal of Materials Research 5(11), 2286-2295.

63. Harris, S. J., Belton, D. N., \& Blint, R. J. (1991). Thermochemistry on hydrogenated diamond $\{111\}$ surface. Journal of Applied Physics 70(5), 26542659.

64. Frenklach, M. \& Spear, K. E. (1988) Growth-mechanism of vapor-deposited diamond Journal of Materials Research 3(1), 133-140.

65. Huang, D., Frenklach, M., \& Maroncelli, M. (1988). Energetics of acetyleneaddition mechanism of diamond growth. Journal of Physical Chemistry 92(22), $6379-6381$.

66. Belton, D. N. \& Harris, S. J. (1992). A mechanism for growth on diamond (110) from acetylene. Journal of Chemical Physics 96(3), 2371-2377.

67. Peploski, J., Thompson, D. L., \& Raff, L. M. (1992). Molecular dynamics studies of elementary surface reactions on of $\mathrm{C}_{2} \mathrm{H}_{2}$ and $\mathrm{C}_{2} \mathrm{H}$ in low-pressure diamondfilm formation. Journal of Physical Chemistry 96(21), 8538-8544. 
68. Brenner, D. W. (1990). Empirical potential for hydrocarbons for use in simulating the chemical vapor deposition of diamond films. Physical Review B, 42 (15), 9458-9471.

69. Larsson, K., Lunell, S., \& Carlsson, J. O. (1993). Adsorption of hydrocarbons on diamond (111) surface - an ab initio quantum mechanical study. Physical Review $B$ 48(4), 2666-2674.

70. Pederson, M. R., Jackson, K. A., Pickett, \& W. E. (1991). Local-densityapproximation-based simulations of hydrocarbon interactions with applications to diamond chemical vapor deposition. Physical Review B 44(8), 3891-3899.

71. Harris, S. J. (1990). Mechanism for diamond growth from methyl radicals, Applied Physics Letters 56(23), 2298-2300.

72. Hamza, A. V., Kubiak, G. D., \& Stulen, R. H. (1988). The role of hydrogen on the diamond C(111)-(2X1) reconstruction. Surface Science 206(1-2), L833-L844.

73. Garrison, B. J., Dawnkaski, E. J., Srivastava, D., \& Brenner, D. W. (1992). Molecular dynamics simulation of dimer opening on diamond (001) (2X1) surface. Science 255(5046), 835-838.

74. Alfonso, D. R., Ulloa, S. E., \& Brenner, D. W. (1994). Hydrocarbon adsorption on diamond (100) stepped surface. Physical Review B 49(7), 4948-4953.

75. Celii, F. G., Pehrsson, P. E., Wang, H. T., \& Butler, J. E. (1988). Infrared detection of gaseous species during the filament-assisted growth of diamond, Applied Physics Letters 52 (24), 2043-2045. 
76. Harris, S. J., Weiner, A. M., \& Perry, T. A. (1988). Measurement of stable species present during filament-assisted diamond growth, Applied Physics Letters, 53 (17), 1605-1607.

77. Hsu, W. L. (1988). Chemical erosion of graphite by hydrogen impact - A summary of the database relevant to diamond film growth. Journal of Vacuum Science and Technology A-Vacuum surfaces and films 6(3), 1803-1811.

78.W. L. Hsu. (1992). Gas-phase kinetics during microwave plasma-assisted diamond deposition - is the hydrocarbon product dictated by neutral-neutral interactions. Journal of Applied Physics, 72(7), 3102-3109.

79. McMaster, M. C., Hsu, W. L., Coltrin, M. E., \& Dandy, D. S. (1994). Experimental measurements and numerical simulations of the gas-compositions in a hot-filament-assisted diamond chemical-vapor-deposition reactor. Journal of Applied Physics 76 (11), 7567-7577.

80. McMaster, M. C., Hsu, W. L., Coltrin, M. E., Dandy, D. S., \& Fox, C. (1995). Dependence of the gas composition in a microwave plasma-assisted diamond chemical vapor deposition reactor on the inlet carbon source: $\mathrm{CH}_{4}$ versus $\mathrm{C}_{2} \mathrm{H}_{2}$. Diamond and Related Materials 4(7), 1000-1008.

81. Rego, C. A., Tsang, R. S., May, P. W., Ashfold, M. N. R. \& Rossner, K. N. J. (1996). Gas-phase composition measurements during chlorine assisted chemical vapor deposition of diamond: A molecular beam mass spectrometric study. Journal of Applied Physics 79(9), 7264-7273.

82. Leeds, S. M., May, P. W., Barlett, E., Ashfold, M. N. R., \& Rosser, K. N. (1999). Molecular beam mass spectrometry studies of gas-phase chemistry occurring 
during microwave plasma assisted chemical vapor deposition of diamond. Diamond and Related Materials 8(8-9), 1377-1382.

83. Aizawa, T., Ando, T., Kamo, M., \& Sato, Y. (1993). High-Resolution electronenergy-loss spectroscopic study of epitaxially grown diamond (111) and (100) surfaces. Physical Review B 48(24) 18348-18351.

84. Lee, S. T. \& Apai, G., (1993) Surface phonons and CH vibrational modes of diamond (100) and (111) surfaces. Physical Review B. 48(4), 2684-2693.

85. Thoms, B. D., Russell, J. N., Pehrsson, P. E., \& Butler, J. E. (1994). Adsorption and abstraction of hydrogen on polycrystalline diamond. Journal of Chemical Physics 100(11), 8425-8431.

86. McGonigal, M, Russell, J. N., Pehrsson, P. E., Maguire, H. G., \& Butler, J. E. (1995). Multiple internal-reflection infrared-spectroscopy of hydrogen adsorbed on diamond (110). Journal of Applied Physics 77(8), 4049-4053.

87. Mankelevich, Y. A., Suetin, N. V., Ashfold, M. N. R., Smith, J. A., \& Cameron, E. (2001). Experimental data vs. 3-D model calculations of HFCVD processes: correlations and discrepancies. Diamond and Related Materials 10(3-7), 364-369.

88. Wulff, G. (1901). Zur Frage der Geschwindigkeit des Wachstums und der Auflösung der. Zeitschrift für Kristallographie, 34, 449-530.

89. Van der Drift, A. (1967). Evolutionary selection, a principle governing growth orientation in vapor-deposited layers. Philips Research Reports 22, 267-288.

90. Clausing, R. E., Heatherly, L., Horton, L. L., Specht, E. D., Begun, G. M., \& Wang, Z. L. (1992). Textures and morphologies of chemical vapor deposited (CVD) diamond. Diamond and Related Materials 1 (5-6), 411-415. 
91. Wild, C., Koidl, P., Mullersebert, W., Walcher, H., Kohl, R., Herres, N., Locher, R., Samlenski, R, \& Brenn, R. (1993). Chemical vapor deposition of smooth (100) faceted diamond films. Diamond and Related materials 2(2-4), 158-168.

92. Tamor, M. A. \& Everson, M. P. (1994). On the role of penetration twins in the morphological development of vapor grown diamond films Journal of Materials Research 9(7), 1839-1848.

93. Menon, P. M., Clausing, R. E. Heatherly, L., Feigerle \& C. S. (1998). The morphology of diamond grown by hot filament chemical vapor deposition. Diamond and related materials 7(8), 1201-1206.

94. Buhler, J. \& Prior, Y. (2000). Study of morphological behavior of single diamond crystals. Journal of crystal growth 209(4), 779-788.

95. Angus, J. C., Buck, F. A., Sunkara, M. K., Groth, T. F., Hayman, C. C. \& Gat, R. (1989), Diamond growth at low pressures. Materials Research Bulletin 10, 38-47.

96. Angus, J. C., Argoitia, A., Gat, R., Li, Z., Sunkara, M. K., Wang, L., \& Wang, Y. (1993) Chemical vapor deposition of diamond, Philosophical transactions of the royal society of London series A: Mathematical, physical and engineering sciences 342 (1664), 195-208.

97. Sunkara, M. K. (1993). Monte-Carlo simulations of diamond nucleation and growth. PhD Thesis, Case Western Reserve University.

98. Angus, J. C., Wang, Y., \& Sunkara, M. (1991). Metastable growth of diamond and diamond-like phases. Annual Review of Materials Science 21, 222-248. 
99. Cleveland, C. L., Landman, U., Schaaff, T. G., Shafigullin, M. N., Stephens, P. W., \& Whetten, R. L. (1997). Structural evolution of smaller gold nanocrystals: The truncated decahedral motif. Physical Review Letters 79(10), 1873-1876.

100. Oku, T. \& Hiraga, K. (2001). Atomic structures and stability of hexagonal $\mathrm{BN}$, diamond and $\mathrm{Au}$ multiply-twinned nanoparticles with five-fold symmetry. Diamond and Related Materials 10 (3-7) 1398-1403.

101. Hayashi, T., Ohno, T., Yatsuya, S., \& Uyeda, R. (1977). Formation of Ultrafine metal particles by gas-evaporation technique. Japanese Journal of Applied Physics 16(5), 705-717.

102. Urban, J. (1998). Crystallography of clusters. Crystal Research and Technology 33(7-8), 1009-1024.

103. Dawnkaski, E. J., Srivastava, D. \& Garrison, B. J. (1995). Time-dependent Monte Carlo simulations of radical densities and distributions on diamond (001)(2X1)/H surfaces. Chemical Physics Letters 232(5-6), 524-530.

104. Xing, J. \& Scott, H. L. (1993). Diamond film growth by chemical vapor deposition - a molecular simulation. Physical Review B 48(7), 4806-4810.

105. Clark, M. M., Raff, L. M., \& Scott, H. L. (1996). Kinetic Monte Carlo studies of early surface morphology in diamond film growth by chemical vapor deposition of methyl radical. Physical Review B 54(8), 5914-5919.

106. Battaile, C. C., Srolovitz, D. J., \& Butler J. E. (1997). A kinetic Monte Carlo method for the atomic-scale simulation of chemical vapor deposition: Application to diamond. Journal of Applied Physics, 82 (12): 6293-6300. 
107. Battaile, C. C., Srolovitz, D. J., Butler, J. E. (1997) Morphologies of diamond films from atomic-scale simulations of chemical vapor deposition. Diamond and Related Materials 6 (9), 1198-1206.

108. Battaile, C. C., Srolovitz, D. J., \& Butler, J. E. (1998). Atomic-scale simulations of chemical vapor deposition on flat and vicinal diamond substrates, Journal of Crystal Growth 194 (3-4), 353-368.

109. Battaile, C. C., Srolovitz, D. J., Oleinik, I. I., Pettifor, D. G., Sutton, A. P., Harris, S. J., Butler, J. E. (1999) Etching effects during the chemical vapor deposition of (100) diamond. Journal of Chemical Physics 111(9), 4291-4299.

110. Bacon, R. (1960). Growth, structure, and properties of graphite whiskers. Journal of Applied Physics 31(2), 283-290.

111. lijima, S. (1991). Helical microtubules of graphitic carbon. Nature $354(6348), 56-58$.

112. Pekala, R. W. (1989). Organic aerogels from the polycondensation of resorcinol with formaldehyde. Journal of Materials Science 24(9), 3221-3227.

113. Yannoni, C. S., Hoinkis, M., de Vries, M. S., Bethune, D. S., Salem, J. R., Crowder, M. S. \& Johnson, R. D. (1992). Scandium Clusters in Fullerene Cages. Science 256(5060), 1191-1192.

114. Shinohara, H., Sato, K., Ohkohchi, M., Ando, Y., Kodama, T., Shida, T., Kato, T., \& Saito, Y. (1992). Encapsulation of Scandium Trimer in C82. Nature $357(6373), 52-54$. 
115. Kuznetsov, V. L., Chuvilin, A. L., Butenko, Y. V., Malkov, I. Y., \& Titov, V. M. (1994). Onion like carbon from ultra-dispersed diamond. Chemical Physics Letters 222(4), 343-348.

116. Dallas, T., Holtz, M., Ahn, H., \& Downer, M. C. (1994). Structural phase of femtosecond-laser-melted graphite. Physical Review B 49(2), 796-801.

117. Itoh, K., Miyahara, Y., Orimo, S., Fujii, H., Kamiyama, T., \& Fukunaga, T. (2003). The local structure of hydrogen storage nanocrystalline graphite by neutron scattering. Journal of Alloys and Compounds 356, 608-611.

118. Shen, T. D., Ge, W.Q., Wang, K.Y., Quan, M.X., Wang, J.T., Wei, W.D., \& Koch, C.C. (1996). Nanostructured Materials 7(4), 393-399.

119. Patterson, J. R., Kudryavtsev, A., \& Vohra, Y. K. (2002). X-ray diffraction and nanoindentation studies of nanocrystalline graphite at high pressures. Applied Physics Letters 81(11), 2073-2075.

120. Haanstra, H.B., Verspui, G., \& Knippenberg, W.F. (1972) Columnar growth of carbon. Journal of Crystal Growth 16(1), 71-79.

121. Ge, M. H. \& Sattler, K. (1994). Observation of fullerene cones. Chemical Physics Letters 220(3-5), 192-196.

122. Krishnan, A., Dujardin, E., Treacy, M. M. J., Hugdah1, J., Lynum, S, \& Ebbesen, T. W. (1997). Graphitic cones and the nucleation of curved carbon surfaces. Nature 388(6641), 451-454. 
123. Iijima, S., Yudasaka, M., Yamada, R., Bandow, S., Suenaga, K., Kokai, F., \& Takahashi, K. (1999). Nano-aggregates of single-walled graphitic carbon nano-horns. Chemical Physics Letters 309 (3-4), 165-170.

124. Gogotsi, Y., Libera, J.A., Kalashnikov, N., \& Yoshimura, M. (2000). Graphite polyhedral crystals. Science 290(5490), 317-320.

125. Gogotsi, Y., Dimovski, S., \& Libera, J.A. (2002) Conical crystals of graphite. Carbon 40(12), 2263-2267.

126. Dong, J., Shen, W., Kang, F., \& Tatarchuk, B. (2002). Whiskers with apex angle $135^{\circ}$ growing by a disclination growth mechanism. Journal of Crystal Growth 245(1-2), 77-83.

127. Tsai, C. L., Chen, C. F., \& Wu, L. K. (2002). Bias effect on the growth of carbon nanotips using microwave plasma chemical vapor deposition. Applied Physics Letters 81(4), 721-723.

128. Goldstein, J. I., Newbury, D., Eclin, P., Joy, D. C., Fiori, C. \& Lufshin, E., (Eds.) (2002). Scanning electron microscopy and X-ray Microanalysis. New York, NY: Plenum Press.

129. Williams, D. B. \& Carter, C. B. (1996). Transmission Electron Microscopy. New York, NY: Plenum press.

130. Cowley, J. M. (1981). Coherent Interference effects in SIEM and CBED. Ultramicroscopy 7(1), 19-26.

131. Cullity, B. D. (Eds.) (1978). Elements of X-ray Diffraction. CA: AddisonWesley Publishing Company, Inc. 
132. Kilaas, R. (2003). Mac Tempas User Manual, Berkeley, CA: Total Resolution.

133. Binnig, G., Quate, C.F., \& Gerber, C. (1986). Atomic force microscope. Physical Review Letters. 56(9), 930-933.

134. Long, D. A. (1977) Raman Spectroscopy. London, UK: McGraw-Hill Inc.

135. Chung, Y. W. (2001). Practical guide to surface science and spectroscopy. San Diego, CA: Academy Press.

136. Wang, J. (Eds.) (2000). Analytical Electrochemistry. New York, NY: Wiley-VCH.

137. Bard, A. J. \& Faulkner, L. R. (Eds.) (2001). Electrochemical Methods. New York, NY: John Wiley \& Sons Inc.

138. Stansbury, E. E., \& Buchanan, R. A. (2000). Fundamentals of Electrochemical Corrosion, Material Park, OH: ASM International.

139. Mitura, S. (1987). Nucleation of diamond powder particles in an RF methane plasma. Journal of Crystal Growth 80(2), 417-424.

140. Buerki, P. R. \& Leutwyler, S. (1991). Homogeneous nucleation of diamond powder by $\mathrm{CO}_{2}$-laser-driven gas-phase reactions. Journal of Applied Physics 69(6), 3739-3744.

141. Pehrsson, P. E., Glesener, J., \& Morrish, A. (1992). Chemical vapor deposition diamond nucleation induced by $\mathrm{sp}^{2}$ carbon on unscratched silicon. Thin Solid Films 212(1-2), 81-90. 
142. Funer, M., Wild, C., \& Koidl, P. (1995). Numerical simulations of microwave plasma reactors for diamond CVD. Surface and Coatings technology, $74-75(1-3), 221-226$.

143. Hassouni, K., Leroy, O., Farhat, S., \& Gicquel, A. (1998). Modeling of $\mathrm{H}_{2}$ and $\mathrm{H}_{2} / \mathrm{CH}_{4}$ moderate-pressure microwave plasma used for diamond deposition. Plasma Chemistry and Plasma Processing 18(3), 325-362.

144. Hwang, N. M. \& Yoon, D. Y. (1996). Thermodynamic approach to the paradox of diamond formation with simultaneous graphite etching in the low pressure synthesis of diamond. Journal of Crystal Growth 160(1-2), 98-103.

145. Cowley, J. M. (1999). Electron nanodiffraction. Microscopy Research and Technique 46(2), 75-97.

146. Raty, J. Y., Galli, G., Bostedt, C., van Buuren, T. W., \& Terminello, L. J. (2003). Quantum confinement and fullerenelike surface reconstructions in nanodiamonds. Physical Review Letters 90(3), 374011-374014.

147. Proserpio, D. N., Hoffmann, R., \& Preuss, P. (1994). Possible hard materials based on interpenetrating diamond-like networks. Journal of American Chemical Society 116(21), 9634-9637.

148. Prawer, S., Peng, J. L., Orwa, J. O., McCallum, J. C., Jamieson, D. N., \& Bursill, L. A. (2000). Size dependence of structural stability in nanocrystalline diamond. Physical Review B 62(24), R16360-R16363.

149. Ree, F. H., Winter, N. W., Glosli, J. N., \& Viecelli, J. A. (1999). Kinetics and thermodynamic behavior of carbon clusters under high pressure and high temperature. Physica B-Condensed Matter 265(1-4), 223-229. 
150. Russo S. P., Barnard A. S., \& Snook I. K. (2003). Hydrogenation of nanodiamond surfaces: Structure and effects on crystalline stability, Surface Review and Letters 10 (2-3): 233-239.

151. Hohenburg, P. \& Kohn, W. (1964) Inhomogeneous electron gas. Physical Review 136(3), B864-B871.

152. Kohn, W. \& Sham, L. J. (1965). Self-consistent equations including exchange and correlation effects. Physical Review 140(4), A1133-A1138.

153. Perdew, J. P., \& Zunger, A. (1981). Self-interaction correction to densityfunctional approximations for many-electron systems. Physical Review B 23(10), 5048-5079.

154. Perdew, J. P. \& Wang, Y. (1992). Pair-distribution function and its coupling-constant average for the spin-polarized electron gas. Physical Review B $46(20), 12947-12954$.

155. Kresse, G. \& Furthmüller, J. (1996). Efficient iterative schemes for $a b$ initio total energy calculations using a plane-wave basis set. Physical Review B 54(16), 11169-11186.

156. Kresse, G. \& Furthmüller, J. (1996). Efficiency of ab initio total energy calculations for metals and semiconductors using a plane-wave basis set. Computational Material Science 6(1), 15-50.

157. Vanderbilt, D. (1990). Soft self-consistent pseudopotentials in generalized eigenvalue formalism. Physical Review B 41(11), 7892-7895.

158. Monkhorst, H. J. \& Pack, J. D. (1976). Special points for Brillouin-zone integrations. Physical Review B 13(12), 5188-5192. 
159. Mailhiot, C. \& McMahan, A. K. (1991). Atmospheric-pressure stability of energetic phases of carbon. Physical Review B 44(21), 11578-11591.

160. Hirai, H., Kondo, K., \& Sugiura, H. (1992). Possible structural models of n-diamond: A modified form of diamond. Applied Physics Letters 61(4), 414-416.

161. Quong, A. A. (1994). Boron hydride analogs of the fullerenes. Physical Review B 50 (7), 4787-4794.

162. Angus, J. C., Sunkara, M. K., Sahaida, S. R., \& Glass, J. T. (1992). Twinning and faceting in early stages of diamond growth by chemical vapor deposition. Journal of Materials Research 7 (11), 3001-3009.

163. Wang, L., Angus, J. C., \& Aue, D. (1995). Morphology of twinned diamond crystals. Journal of Materials Research 10 (12), 3037-3040.

164. Fichthorn, K. A. \& Weinberg, W. H. (1991). Theoretical foundation of dynamic Monte Carlo simulations. Journal of Chemical Physics 95(2), 10901096.

165. Shechtman, D., Hutchison, H. L., Robins, L. H., Farabaugh, E. N., \& Feldman, A. (1993). Growth defects in diamond films. Journal of Materials Research 8 (3) 473-479.

166. Dorignac, D., Delclos, S., \& Phillipp, F. (2001). Atomic structure of a complex defect configuration in synthetic diamond: a fivefold twin centre connected to two high-order grain boundaries. Philosophical Magazine BCondensed Matter Statistical Mechanics Electronic Optical and Magnetic Properties 81(11), 1879-1891.

167. Angus, J. C. \& Dyble, T. J. (1975). Surface Science 50(1) 157-177.

168. van Veenendaal, E., de Theije, F. K., van Suchtelen, J., van Enckevort, W. J. P. (2002). Simulation of step patterns on natural diamond $\{111\}$ surfaces. Diamond and Related Materials 11(2), 145-152. 
169. Battaile, C. C., Srolovitz, D. J., Oleinik, I. I., Pettifor, D. G., Sutton, A. P., Harris, S. J., Butler, J. E. (1999). Etching effects during the chemical vapor deposition of (100) diamond. Journal of Chemical Physics 11(9), 4291-4299.

170. Angus, J. C. \& Hayman, C. C. (1988). Low-pressure, metastable growth of diamond and diamond-like phases. Science 241(4868), 913-921.

171. Frenklach, M. (1992). Monte Carlo simulation of diamond growth by methyl and acetylene reactions. Journal of Chemical Physics 97(8), 5794-5802.

172. Colineau, E., Gheeraert, E., Deneuville, A., Mambou, J., Brunet, F. \& Lagrange, J. P. (1997). Minimization of the defects concentration from boron incorporation in polycrystalline diamond films. Diamond and Related Materials $6(5-7), 778-782$.

173. Huang, S. M., Sun, Z., Lu, Y. F., \& Hong, M. H. (2002). Ultraviolet and visible Raman spectroscopy characterization of chemical vapor deposition diamond films. Surface and Coatings Technology 151, 263-267.

174. Ferrari, A. C. \& Robertson, J. (2000). Interpretation of Raman spectra of disordered and amorphous carbon. Physical Review B 61(20), 14095-14107.

175. Matthews, M. J., Pimenta, M. A., Dresselhaus, G., Dresselhaus, M. S., \& Endo, M. (1999). Origin of dispersive effects of the Raman D band in carbon materials. Physical Review B 59(10), R6585-R6588.

176. Amelinckx, S., Luyten, W., Krekels, T., Van Tendeloo, G., \& Van Landuyt, J. (1992). Conical, helically wound, graphite whiskers - A limiting number of the fullerenes. Journal of Crystal Growth 121(4), 543-558. 
177. Kissinger, P. T. \& Heineman, W. R. (Eds.) (1984). Laboratory techniques in electroanalytical chemistry. New York, NY: Marcel Dekker Inc.

178. Adams, R. N. (1958). Carbon Paste Electrodes. Analytical Chemistry 30(9), 1576-1576.

179. Miller, F. J. \& Zittel, H. E. (1968). Fabrication and use of pyrolytic graphite electrode for voltammetry in aqueous solutions. Analytical Chemistry 36(9), 1866-1869.

180. Zittel, H. E. \& Miller, F. J. (1965). A glassy carbon electrode for voltammetry. Analytical Chemistry 37(5), 200-203.

181. Xu, J. S., Granger, M. C., Chen, Q. Y., Strojek, J. W., Lister, T. E., \& Swain, G. M. (1997). Analytical Chemistry 69(19), A591-A597.

182. Martin, H. B., Argoitia, A., Landau, U., Anderson, A. B. \& Angus, J. C. (1996). Hydrogen and oxygen evolution on boron-doped diamond electrodes. Journal of the Electrochemical Society 143(6), L133-L136.

183. Martin, H. B., Argoitia, A., Angus, J. C., \& Landau, U. (1999). Voltammetry studies of single-crystal and polycrystalline diamond electrodes. Journal of the Electrochemical Society 146(8), 2959-2964.

184. Swain, G. M., Anderson, A. B., \& Angus, J. C. (1998). Applications of diamond thin films in electrochemistry. MRS Bulletin 23(9), 56-60.

185. Tenne, R. \& Clement, C. L. (1998). Diamond electrodes. Israel Journal of Chemistry 38(1-2), 57-73. 
186. Yano, T., Tryk, D. A., Hashimoto, K., \& Fujishima, A. (1998). Electrochemical behavior of highly conductive boron-doped diamond electrodes for oxygen reduction in alkaline solution. Journal of the Electrochemical Society 145(6), $1870-1876$.

187. Show, Y., Witek, M. A, Sonthalia, P., \& Swain, G.M. (2003). Characterization and electrochemical responsiveness of boron-doped nanocrystalline diamond thin-film electrodes. Chemistry of Materials 15(4), 879888.

188. Hian, L.C., Grehan, K.J., Compton, R.G., Foord, J.S., \& Marken, F. J. (2003). Nanodiamond thin films on titanium substrates - Growth and electrochemical properties. Journal of the Electrochemical Society 150(1), E59E65.

189. Chen, Q. Y., Gruen, D. M., Krauss, A. R., Corrigan, T. D., Witek, M., \& Swain, G. M. (2001). The structure and electrochemical behavior of nitrogencontaining nanocrystalline diamond films deposited from $\mathrm{CH}_{4} / \mathrm{N}_{2} / \mathrm{Ar}$ mixtures. Journal of the Electrochemical Society 148(1), E44-E51.

190. Chen, P. H. \& McCreery, R. L. Control of electron transfer kinetics at glassy carbon electrodes by specific surface modification. Analytical Chemistry 68(22), 3958-3965.

191. Michael, A. C. \& Wightman, R. M. (1996). Microelectrodes. Laboratory Techniques Electroanalytical Chemistry New York, NY: Marcel Dekker Inc. 
192. Penner, R. M., Heben, M. J., Longin, T. L., \& Lewis, N. S. (1990). Fabrication and use of nanometer-sized electrodes in electrochemistry. Science $250(11), 1118-1121$.

193. Britto, P. J., Santhanam, K. V. S., Rubio, A., Alonso, J. A., \& Ajayan, P. M. (1999). Improved charge transfer at carbon nanetube electrodes. Advanced Materials 11(2), 154-157.

194. Nugent, J. M., Santhanam, K. V. S., Rubio, A., \& Ajayan, P. M. (2001) Fast electron transfer kinetics on multiwalled carbon nanotube microbundle electrodes, Nano Letters 1(2), 87-91.

195. Li, J., Cassell, A., Delzeit, L., Han, J. and Meyyappan, M. (2002) Novel three-dimensional electrodes: Electrochemical properties of carbon nanotube ensembles. Journal of Physical Chemistry B 106(36), 9299-9305.

196. Koehne, J., Li, J., Cassell, A. M., Chen, H., Ye, Q., Ng, H. T., Han, J., \& Meyyappan, M. (2004). The fabrication and electrochemical characterization of carbon nanotube nanoelectrode arrays. Journal of Materials Chemistry 14(4), 676-684.

197. Valentini, F., Amine, A., Orlanducci, S., Terranova, M. L., \& Palleschi, G. (2003). Carbon nanotube purification: Preparation and characterization of carbon nanotube paste electrodes. Analytical Chemistry 75(20), 5413-5421.

198. Wang, Z. H., Liu, J., Liang, Q. L., Wang, Y. M., \& Luo, G. (2002). Carbon nanotube-modified electrodes for the simultaneous determination of dopamine and ascorbic acid. Analyst 127(5), 653-658. 
199. Tuinstra, F. \& Koenig, J. L. (1970). Raman spectrum of graphite. Journal of Chemical Physics 53(3), 1126-1130.

200. Katagiri, G., Ishida, H., \& Ishitani, A. (1988). Raman spectra of graphite edge planes. Carbon 26(4), 565-571.

201. Nemanich, R. J., Glass, J. T., Lucovsky, G., \& Shroder, R. E. (1988). Raman-scattering characterization of carbon bonding in diamond and diamondlike thin films. Journal of Vacuum Science and Technology A-Vacuum surfaces and films 6(3), 1783-1787.

202. Gratzel, M. (2001). Photoelectrochemical cells. Nature 414(6861), 338344.

203. Ranganathan, S., Kuo, T. C., \& McCreery, R. L. (1999). Facile preparation of active glassy carbon electrodes with activated carbon and organic solvents. Analytical Chemistry 71(16), 3574-3580.

204. Kneten, K. R. \& McCreery, R. L. (1992). Effects of redox system structure on electron-transfer kinetics at ordered graphite and glassy carbon electrodes. Analytical Chemistry 64(21), 2518-2524.

205. Venton, B. J., Wightman, R. M. (2003). Psychoanalytical Electrochemistry: Dopamine and behavior. Analytical Chemistry 75(19), 414A$421 \mathrm{~A}, 2003$ 
206. Deakin, M. R. \& Wightman, R. M. (1986). The kinetics of some substituted catechol/ortho-quinone couples at carbon paste electrode. Journal of Electroanalytical Chemistry 206(1-2), 167-177.

207. Cabaniss, G. E., Diamantis, A. A., Murphy, W. R., Linton, R. W., \& Meyer, T. J. (1985). Electrocatalysis of proton-coupled electron transfer reactions at glassy carbon electrodes. Journal of the American Chemical Society 107(7), 1845-1853.

208. DuVall, S. H. \& McCreery, R. L. (2000). Self-catalysis by catechols and quinones during heterogeneous electron transfer at carbon electrodes. Journal of the American Chemical Society 122(28), 6759-6764.

209. Bowling, R., Rackard, R. T., \& McCreery, R. L. (1986). Mechanism of electrochemical activation of carbon electrodes - role of graphite lattice-defects. Langmuir 5(3), 683-688.

210. Bradley, R. H., Ling, X., \& Sutherland, I. (1993). An investigation of carbon-fiber surface-chemistry and reactivity based on XPS and surface freeenergy. Carbon 31(7), 1115-1120.

211. Biniak, S., Szymanski, G., Siedlewski, J., \& Swaitowski, A. (1997). The characterization of activated carbons with oxygen and nitrogen surface groups. Carbon 35(12), 1799-1810.

212. Nakahara, M., Nakayama, Y., Katagiri, G., \& Shimizu, K. (1991). Anodicoxidation effects on pyrolytic graphite surfaces in acid. Journal of Materials Science 26(4), 861-864. 
213. Rice, R. J. \& McCreery, R. L. (1989). Quantitative relationship between electron-transfer rate and surface microstructure of laser-modified graphite electrodes. Analytical Chemistry 61(15), 1637-1641.

214. Regisser, F., Lavoie, M. A., Champagne, G. Y., \& Belanger, D. (1996). Randomly oriented graphite electrode. Part 1. Effect of electrochemical pretreatment on the electrochemical and chemical composition of the electrode. Journal of Electroanalytical Chemistry 415 (1-2), 47-54.

215. Notsu, H., Yagi, I., Tatsuma, T., Tryk, D.A., \& Fujishima, A. (2000) Surface carbonyl groups on oxidized diamond electrodes. Journal of Electroanalytical Chemistry 492(1), 31-37.

216. Popa, E., Notsu, H., Miwa, T., Tryk, D. A., \& Fujishima, A. (1999). Selective electrochemical detection of dopamine in the presence of ascorbic acid at anodized diamond thin film electrodes. Electrochemical and Solid State Letters 2(1), 49-51.

217. Sarada, B. V., Rao, T. N., Tryk, D. A., \& Fujishima, A. (2000). Electrochemical oxidation of histamine and serotonin at highly boron doped diamond electrodes. Analytical Chemistry 72(7), 1632-1638.

218. Falat, L. \& Cheng, H. Y. (1982). Voltammetric differentiation of ascorbic acid and dopamine at electrochemically treated graphite epoxy electrode. Analytical Chemistry 54(12), 2108-2111. 
219. Hu, I. F. \& Kuwana, T. (1986). Oxidative mechanism of ascorbic acid at glassy carbon electrodes. Analytical Chemistry 58(14), 3235-3239.

220. Heinze J. (1993). Utramicroelectrodes in electrochemistry. Angewandte Chemie - International Addition in English 32(9), 1268-1288.

221. Oku, T. \& Hiraga, K. (2000). Atomic structures and stability of hexagonal $\mathrm{BN}$, diamond and Au multiply-twinned nanoparticles with five-fold symmetry. Diamond and Related Materials 10(3-7), 1398-1403.

222. Sweegers, C., de Coninck, H.C., Meekes, H., van Enckevort, W.J.P., Hiralal, I.D.K., \& Rijkeboer, A. (2001). Morphology, evolution and other characteristics of gibbsite crystals grown from pure and impure aqueous sodium aluminate solutions. Journal of Crystal Growth 233(3), 567-582.

223. Prausnitz, M. R. (2004). Microneedles for transdermal drug delivery, Advanced Drug Delivery Reviews 56(5), 581-587. 


\section{APPENDIX 1}

\section{PRIMARY PLANES OF DIAMOND}

\section{(1) Diamond $\{100\}$ surface}

This plane is characterized by atoms bonded twice to the surface. Only one atom is needed to form a step on $\{100\}$. The interplanar spacing in $\{100\}$ is equal to the lattice parameter of diamond, i.e. $0.3567 \mathrm{~nm}$. Because of their symmetry, these surfaces are generally square in shape.

Atoms bonded twice to the surface on $\{100\}$

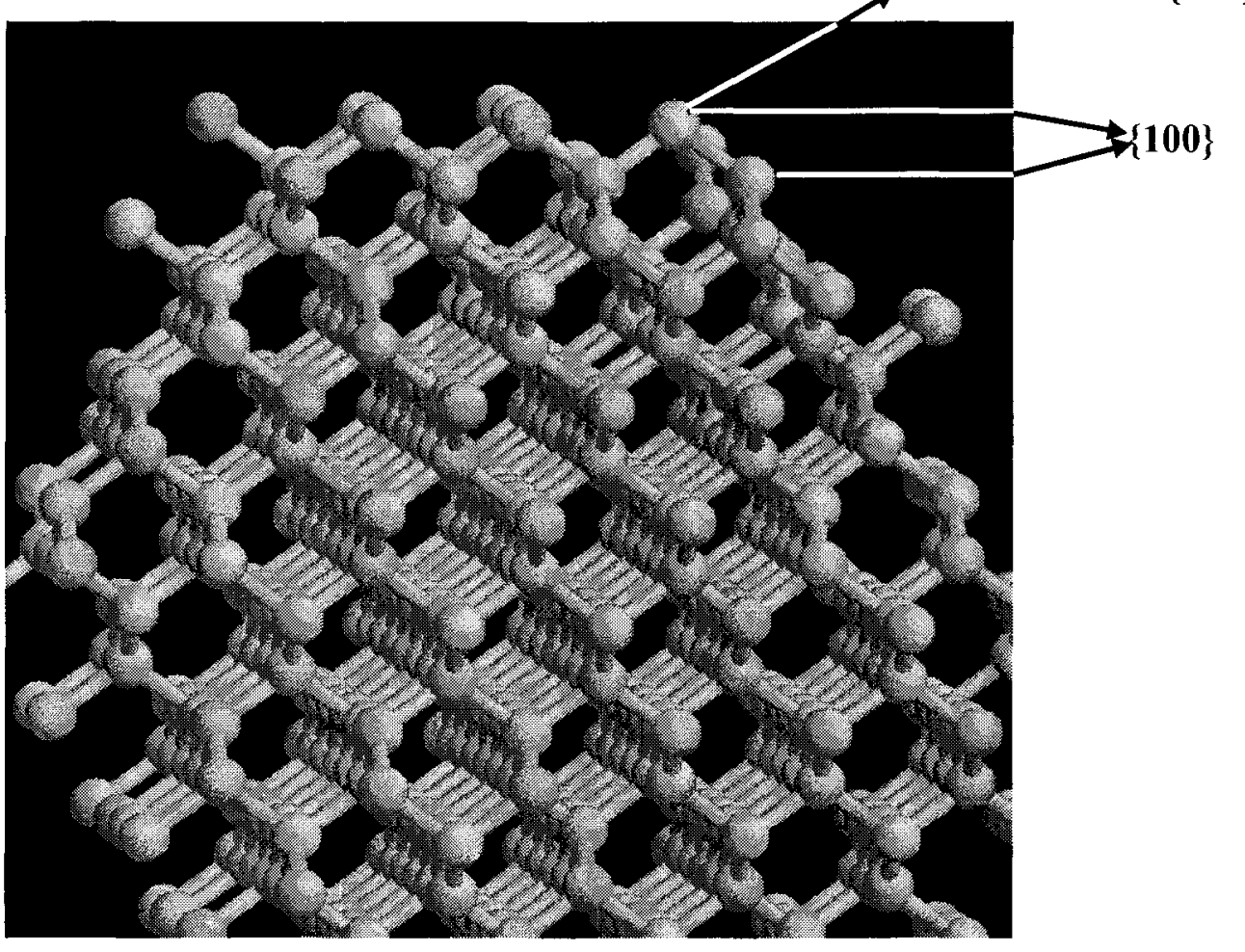

Figure A1.1 Ball and stick model of the diamond $\{100\}$ plane. 


\section{(2) Diamond $\{111\}$ surface}

Diamond $\{111\}$ planes are characterized by atoms that are either bonded twice to the surface or atoms that are bonded once to the surface. Both these are indicated in Figure A1.2. The distance between each $\{111\}$ plane of diamond is $0.206 \mathrm{~A}$. The stacking of atoms is $\mathrm{ABC}$. At least three atoms are needed to form a stable cluster on $\{111\}$ that is not capable of rotation. Because of their symmetry, these surface are generally triangular.
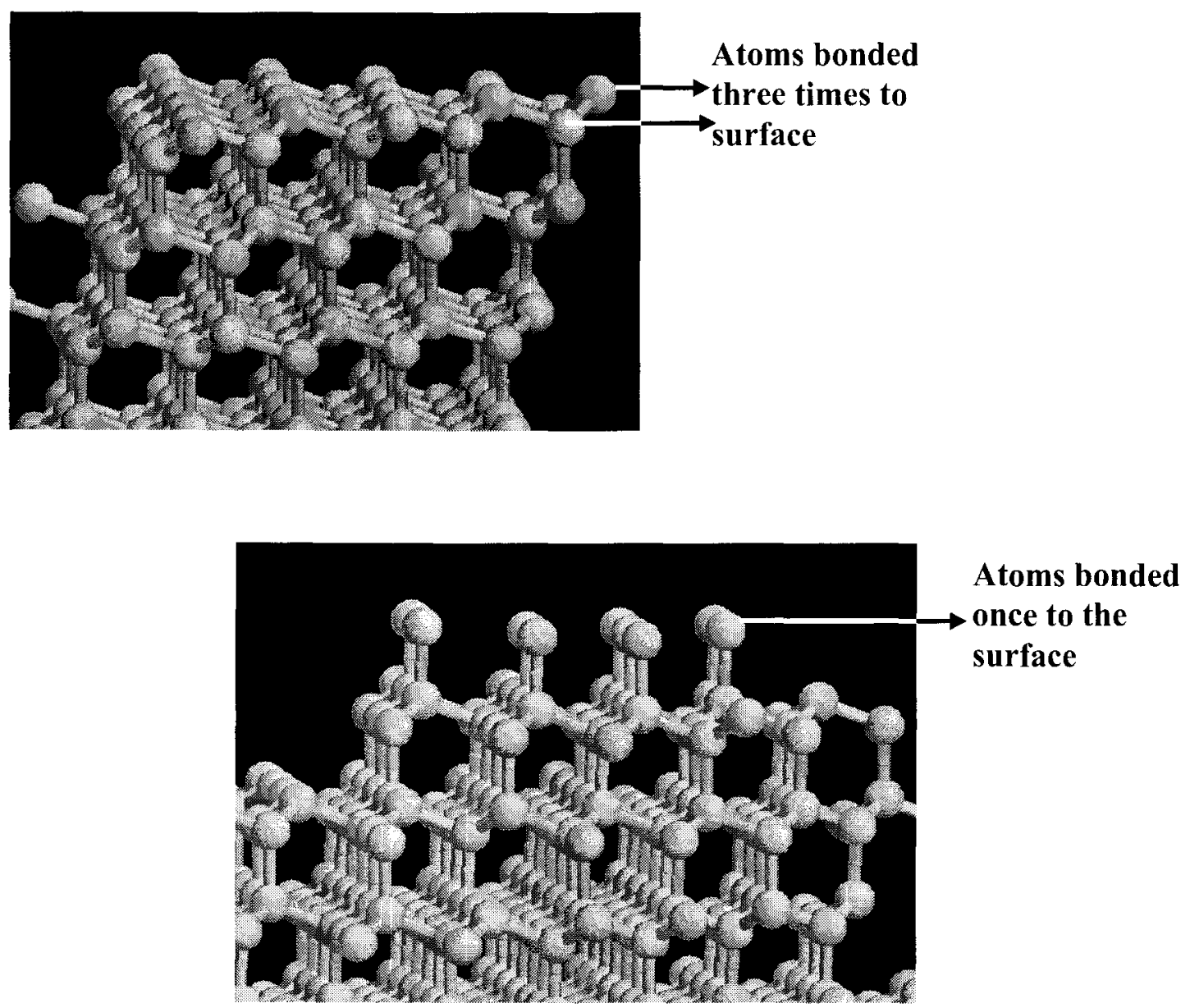

Figure A1.2 Ball and stick model of the two types of $\{111\}$ planes with atoms bonded (a) thrice to the surface (b) with atoms bonded only once to the surface. 
(3) Diamond $\{110\}$ surface

The $\{110\}$ directions is considered the fastest growth direction, hence at the end of diamond growth $\{110\}$ planes are rarely seen. The surface is generally dominated by $\{100\}$ and $\{111\}$ planes. To form a stable step on $\{110\}$, at least 3 atoms are needed. These planes have a rectangular shape. And atoms are bonded 3 times to the surface.

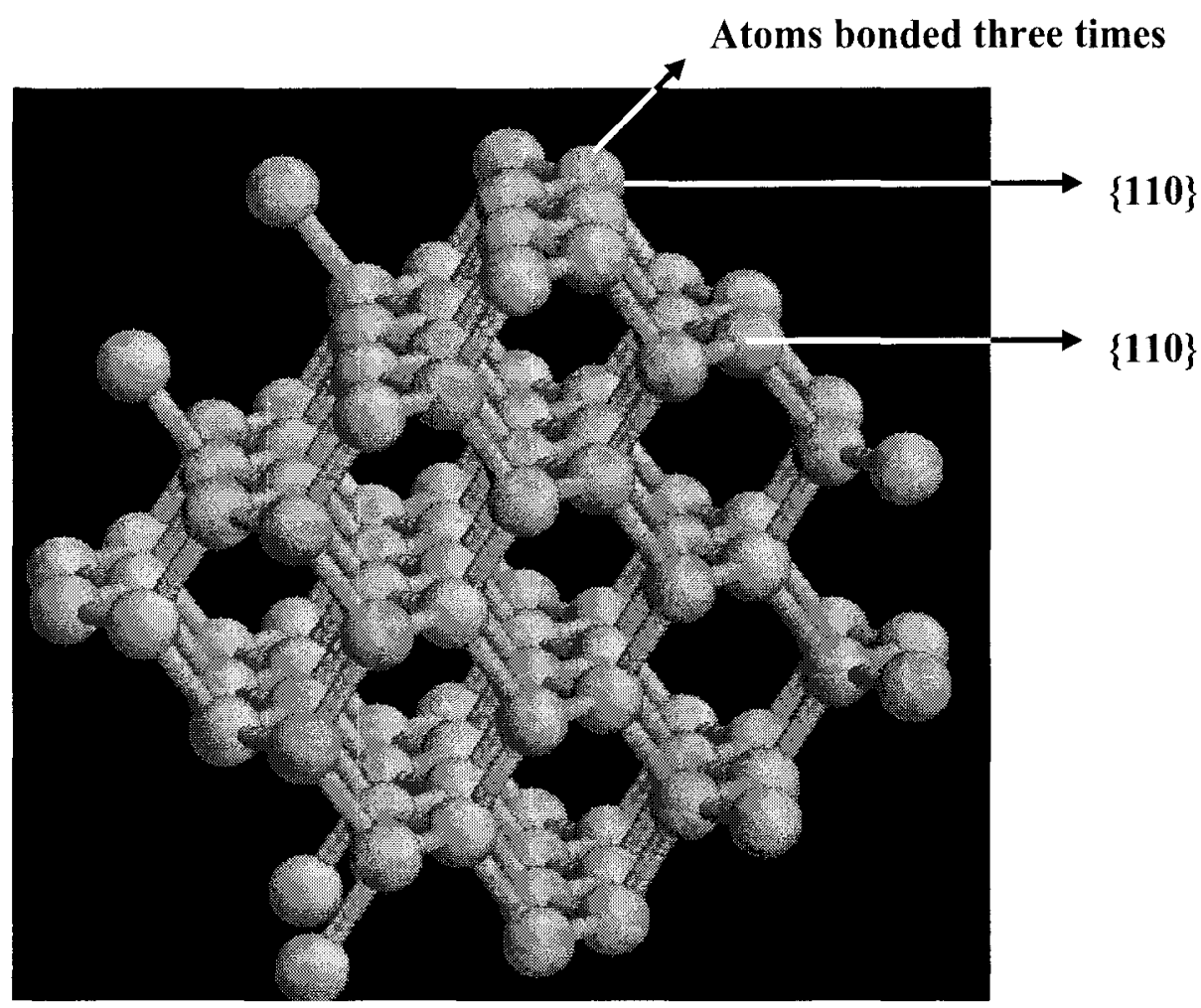

Figure A1.3 Ball and stick model of diamond $\{110\}$ plane. 


\section{APPENDIX 2}

\section{SEQUENCE OF DECAHEDRON CONVERTING TO ICOSAHEDRON}

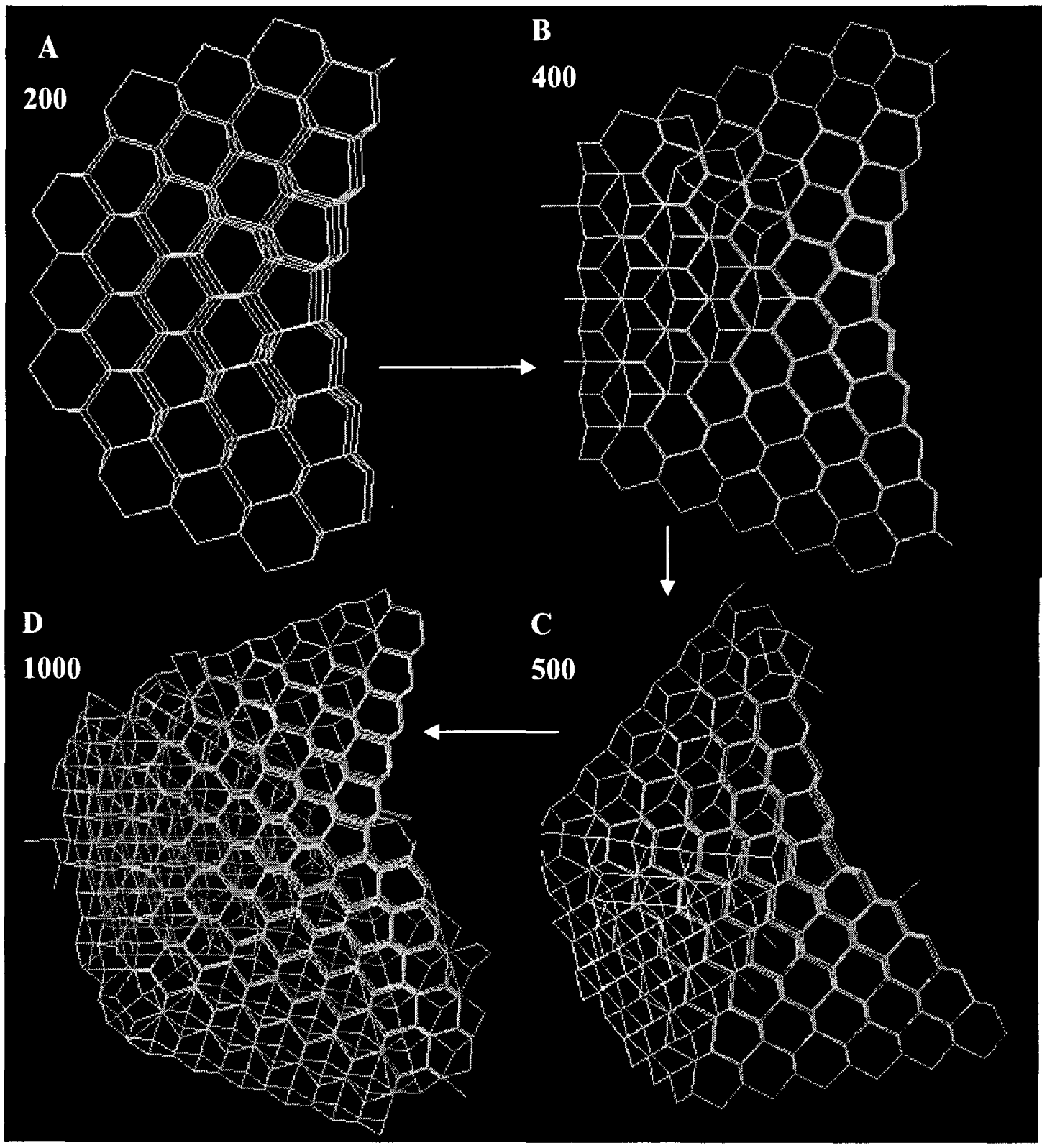

A2.1 (a-d) Wire-frame model of a simulated sequence of five-fold twinned crystals converting to an icosahedral crystal by the addition of a stacking fault during growth. 
APPENDIX 3-DECAHEDRAL AND STAR-DECAHEDRAL SIMULATIONS
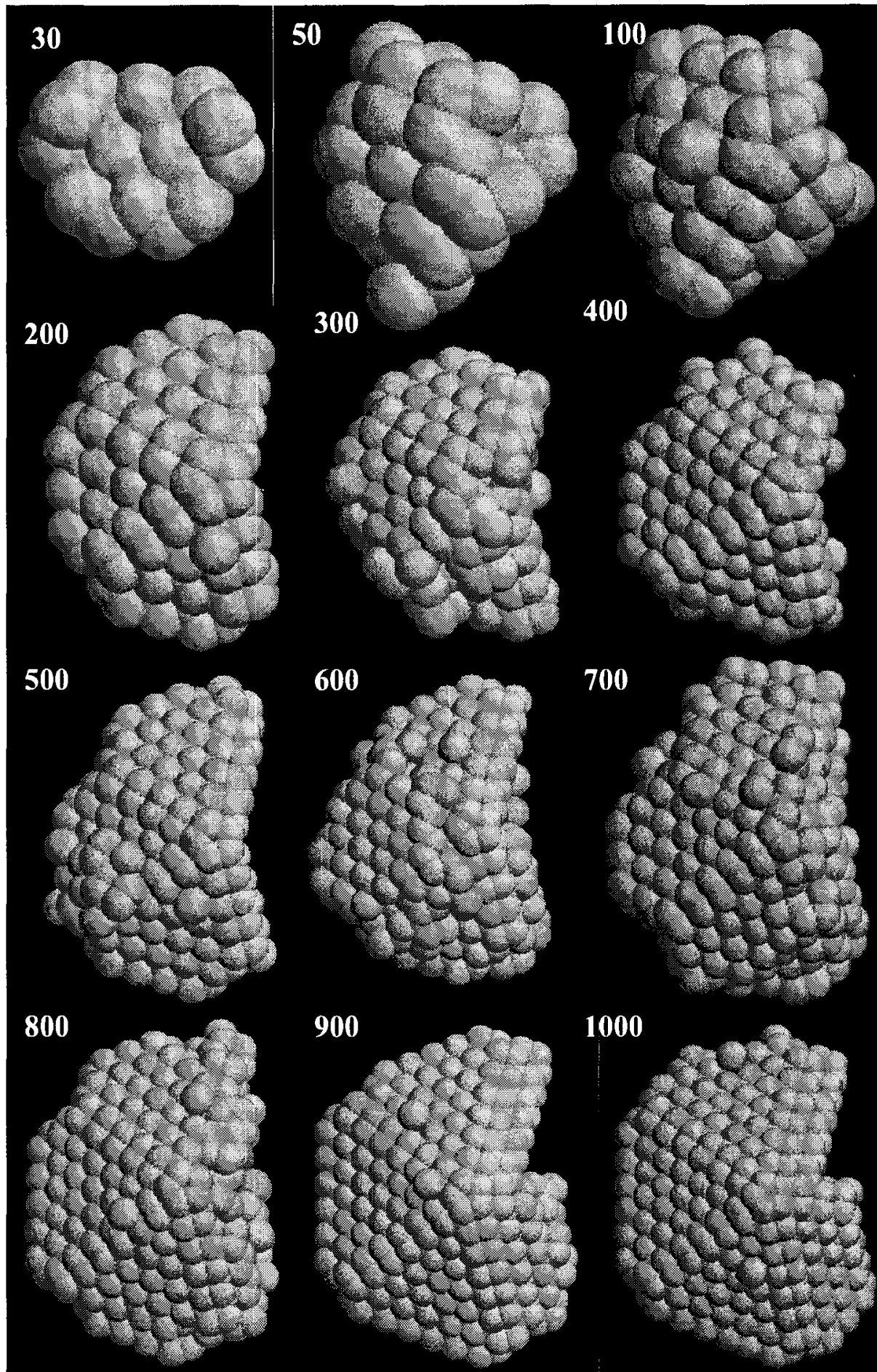

900

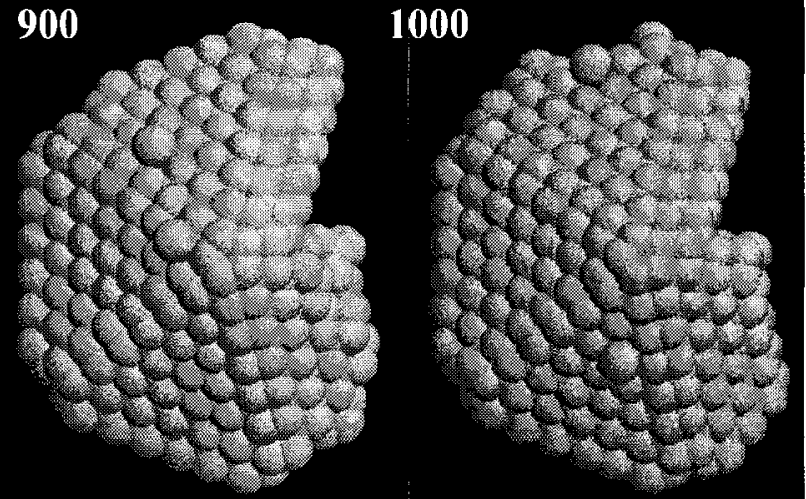

A 3.1 Space-filled model of the initial stages of decahedron growth till 1000 atoms. 


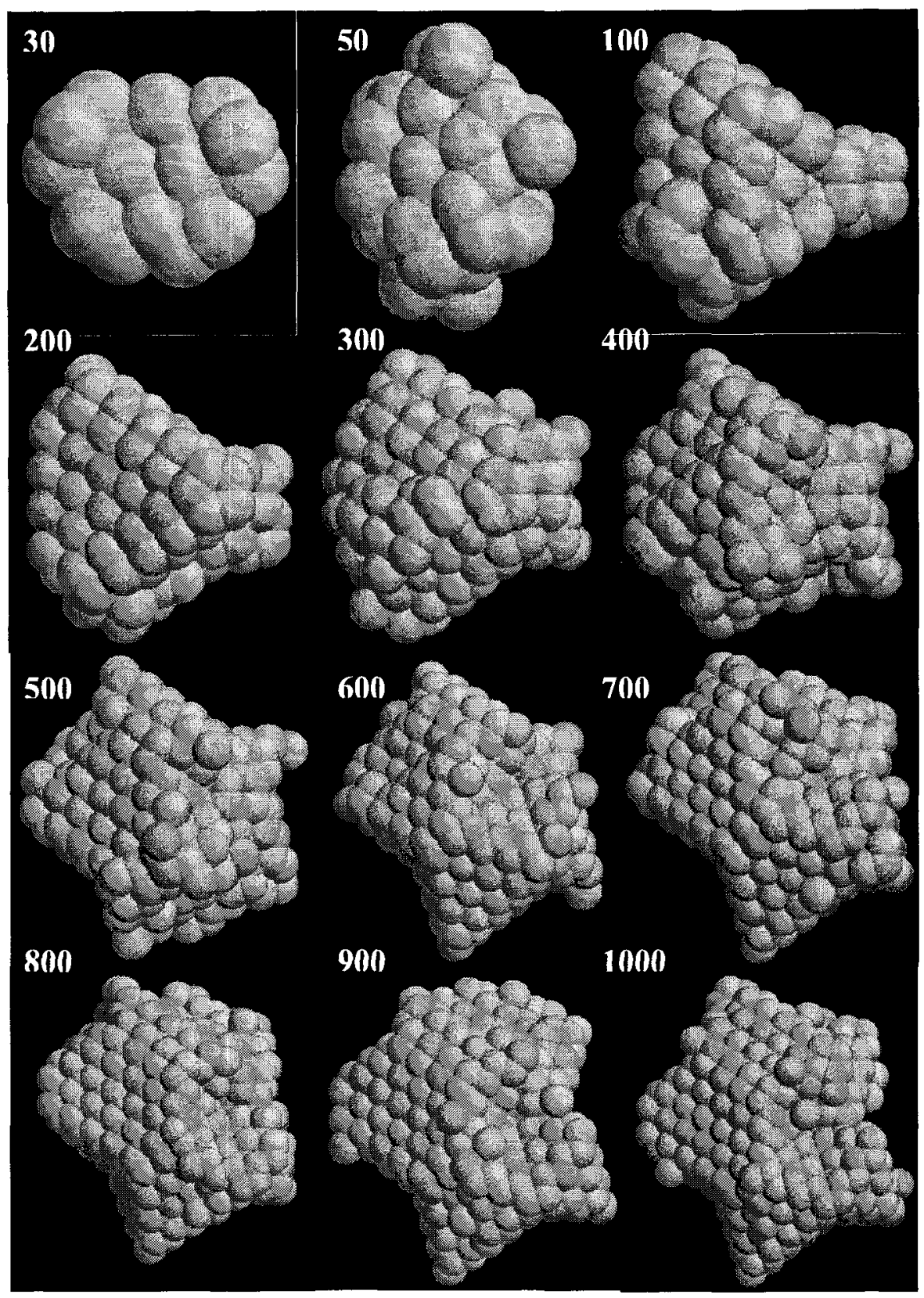

A 3.2 Space-filled model of the initial stages of star-decahedron growth till 1000 atoms. 


\section{CURRICULUM VITAE}

NAME: $\quad$ Radhika C. Mani

ADDRESS: Department of Chemical Engineering

University of Louisville

Louisville, KY 40292

DOB: $\quad$ Mithapur, India- August 17, 1978.

EDUCATION

\& TRAINING: B.E, Chemical Engineering

Maharaja Sayajirao University, Baroda, India

1996-2000

$\mathrm{PhD}$, Chemical Engineering

University of Louisville

2000-2004

ACADEMIC

Who's Who in American Colleges and Universities 2002

Government Scholarship for being one of the top 100 students in the State in High School (1996)

Principal's best student award in High School (1996)

PROFESSIONAL SOCIETIES: American Institute of Chemical Engineers

Materials Research Society

PUBLICATIONS: R. C. Mani, X. Li, M.K. Sunkara, K. Rajan, "Carbon Nanopipettes", Nano Letters; 3(5), 671, (2003).

R. C. Mani and M. K. Sunkara, "Kinetic Faceting of Multiply Twinned Diamond Crystals during Vapor Phase Synthesis", Diamond and Related Materials; 12, 324, (2003).

R. C. Mani, S. Sharma, M. K. Sunkara, J. Gullapalli, R. P. Baldwin, R. Rao, A. M. Rao, J. M. Cowley, "Synthesis and Electrochemical Characteristics of a Nanocomposite Diamond 
Electrode", Electrochemical and Solid-State Letter; 5(6), E32 (2002).

S. Sharma, H. Li, H. Chandrasekaran, R.C. Mani, M.K. Sunkara, "Synthesis of Inorganic Nanowires and Nanotubes", An invited chapter in the Encyclopedia of Nanoscience and Nanotechnology; Edited by H. Nalwa, American Scientific Publishers, Los Angeles, CA, 10, 373(2003).

R. C. Mani, M. K. Sunkara, R. P. Baldwin, "Carbon Nanopipettes: Synthesis and Electrochemical properties", NATO-ASI Nanoengineered and Nanofibrous Materials Proceedings, Antalya, Turkey, (September 1-11 2003), Edited by S. Guceri, V. Kuznetsov, Y. Gogotsi, Kulwer Press, Dordrecht, NL (May 2004).

R. C. Mani, M. K. Sunkara, R. P. Baldwin, "Carbon Nanopipettes: Electrochemical Sensors for neurological fluids", 198f, AICHE annual meeting, San Francisco, USA (Nov 2003).

G. Bhimarasetti, R. C. Mani, M. K. Sunkara, K. Rajan, X. Li, "Novel Carbon Nanostructures: Nanopipettes and Nanonozzles", Proceedings of the 7th ADC/FCT Conference, A15, Tsukuba, Japan, (August 2003).

US PATENTS: $\quad$ R. C. Mani, S. Sharma, M. K. Sunkara, J. Gullapali, R. P. Baldwin, "A novel electrode material system (Glassy Diamond) for neurological and bio-medical sensing applications: Synthesis method and electrochemical characteristics", Invention Disclosure Filed (July 2001).

M. K. Sunkara, R. C. Mani, C. Paterson, "Carbon Nanopipettes: Synthesis and Applications", Patent pending (April 2003).

NATIONAL

MEETINGS

PRESENTATIONS: R. C. Mani, M. K. Sunkara, R. P. Baldwin, "Carbon nanopipettes; Synthesis and electrochemical properties", Oral presentation at the NATO-ASI meeting in September 2003, Antalya, Turkey.

R. C. Mani, M. K. Sunkara, "Kinetic Monte Carlo simulations for star-decahedral diamond crystals", Oral presentation at the AIChE Annual Meeting in November 2003, San Francisco, CA. 
R. C. Mani, M. K. Sunkara, R. P. Baldwin, "Carbon Nanopipettes: Electrochemical sensors for neurological fluids", Oral presentation at the AIChE Annual meeting in November 2003, San Francisco, CA.

R. C. Mani, M. K. Sunkara, "Kinetic faceting of multiply-twinned diamond crystals", Poster presentation at the MRS Fall meeting in December 2002, Boston, MA.

R. C. Mani, M. K. Sunkara, J. Gullapalli, R. P. Baldwin, “A novel nanocomposite diamond electrode for electrochemical sensing of neurological fluids", Oral presentation at the AIChE annual meeting in November 2002, Indianapolis, IN.

R. C. Mani, S. Sharma, M. K. Sunkara, J. Gullapalli, R. P. Baldwin, K. McGuire, R. Rao, A. M. Rao, J. M. Cowley, "Nanocomposite Films and Free-standing Whiskers Containing Diamond and Graphite phases", Poster Presentation at the Diamond 2002 European conference in September 2002, Granada, Spain.

INVITED

PRESENTATIONS: R. C. Mani, "Structural and morphological manifestations of nanocrystalline carbon phases", Invited talk at Los Alamos National Laboratory in February 2004, Los Alamos, NM.

R. C. Mani, G. Bhimarasetti, M. K. Sunkara, R. P. Baldwin, C. Paterson, "Nanopipettes and micropipes: Durg delivery patches, ink-jet heads and neurochem electrodes", Invited talk at the KYNanomat conference in September 2003, Louisville, KY. 